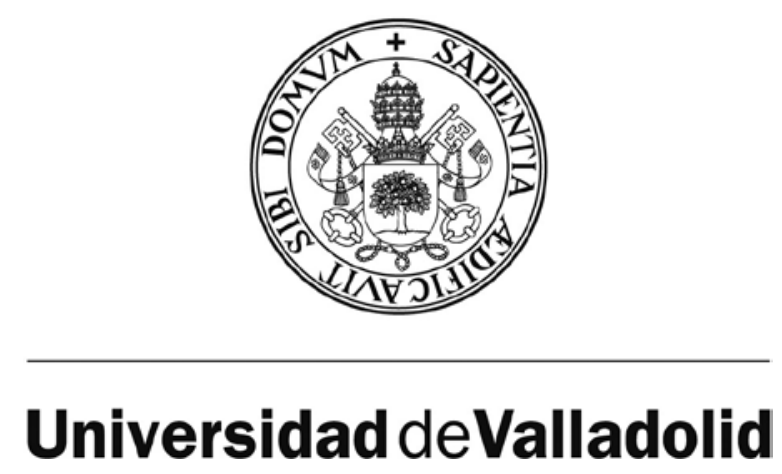

ESCUELA DE INGENIERÍAS INDUSTRIALES

DPTO. DE INGENIERÍA DE SISTEMAS Y AUTOMÁTICA

TESIS DOCTORAL:

\title{
A STUDY ON \\ MODELLING, DATA RECONCILIATION AND OPTIMAL OPERATION OF HYDROGEN NETWORKS IN OIL REFINERIES
}

Presentada por Elena $\mathrm{M}^{\mathrm{a}}$ Gómez Sayalero para optar al grado de doctora por la Universidad de Valladolid

Dirigida por:

Prof. Dr. César de Prada Moraga 



\section{ACKNOWLEDGEMENTS}

I would like to express my profound gratitude to my thesis director, Prof. Ph.D. César de Prada Moraga, for his trust, guidance and support, as well as for the great opportunity he offered me.

This work have been possible thanks to the cooperation and involvement of Petronor ${ }^{\circledR}$ and Repsol ${ }^{\circledR}$ companies, in particular the following Departments: Petronor Process Engineering (Rafael González Martín, Carlos Pascual Román), Petronor Advanced Control (José Miguel Sola Sáez, Sergio Mármol Gutiérrez, Izaskun Bartolomé Lacarra, and in the last stage also Roberto González Apellániz), Petronor Production (Elías Unzueta Etxeita, José Mạ Álvarez García, José Ramón Lacarra, Jáuregui), Repsol CTR (Ana Alcalde Bascones, Benito Caamaño Nieves, Patricia Soriano Ferrero), Repsol Energy Management (Antonio López Rodríguez, Marta Yugo Santos) and Repsol CTR Technology (Miguel Ángel García Carreño); the collaboration was challenging, stimulating and pleasant.

Financial support of the Spanish Ministry of Science and Innovation (CICYT MICINN DPI200613593 "Optimal operation of process plants"; CICYT MICINN FPI program under grant BES2007-15683; CICYT MICINN DPI2009-12805 "Control and optimization of distribution networks"), as well as funding from the European Union Seventh Framework Programme FP7 (HYCON2 under grant agreement 257462; MORE Monitoring Resource Efficiency under grant agreement 604068) are gratefully acknowledged.

The short stay at the Process Control Group under the supervision of Prof. Ph.D. Sigurd Skogestad in the Department of Chemical Engineering at the Norwegian University of Science and Technology (NTNU) in Trondheim, Norway, also his guidance and feedback, as well as the few days spent at the Process Dynamics and Operations Group under the supervision of Prof. Ph.D. Sebastian Engell in the Biochemical-Chemical Engineering Faculty at the Technical University of Dortmund, Germany, and equally his guidance and feedback, are truly appreciated.

I would like to extend my appreciation to Prof. Ph.D. Gerardo González Benito, from the Chemical Engineering Department, and to all the Lecturers, staff and colleagues at the Systems Engineering and Automation Department (Mergelina building) of the Industrial Engineering School of the University of Valladolid, for their support and assistance, in particular to Ph.D. Jesús Ma Zamarreño Cosme and Ph.D. Luis Felipe Acebes Arconada; to Ph.D. Almudena Rueda 
Ferreiro and Ph.D. Alejandro Merino Gómez, whose work at the Sugar Technology Center (CTA) proved an inestimable reference; to Jacobo $M$. Salamanca Parra for his help while configuring the Edusca ${ }^{\circledR}$ SCADA and CreaOPC ${ }^{\circledR}$ tools developed by Ph.D. Raúl Alves Santos; to David Marina, José Antonio Caminero Granja and Carlos Gómez Palacín for their willingness while implementing the link libraries they developed; as well as to Ph.D. Gloria Gutiérrez Rodríguez, Ph.D. Smaranda Cristea, Ph.D. Ma Jesús de la Fuente, Ph.D. Miguel Ángel García, Ph.D. Rogelio Mazaeda, Mar Valbuena, Ph.D. Daniel Sarabia, Miguel Rodríguez, Ph.D. José Luis Pitarch, and Teresa de Jesús Álvarez -thanks a lot for all the installations-.

Finally, all my thankfulness to CTA colleagues and friends -best memories from the daily coffee and other shared moments, moreover for motivating conversations- and friends from Valladolid -specially to Ph.D. Marta D., Ph.D. Almudena and Ph.D. Mónica- and from Viloria del Henar, for their invaluable encouragement and kindness, as well as to my family who gave me everything. 


\section{CONTENTS}

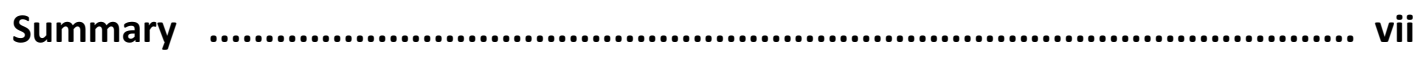

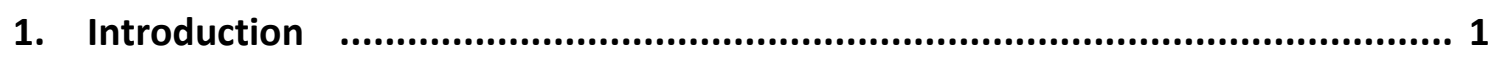

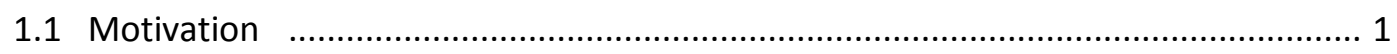

1.2 State of the art

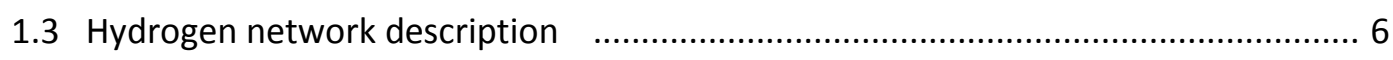

1.3.1 General hydrodesulfurizer HDS plant description .................................... 8

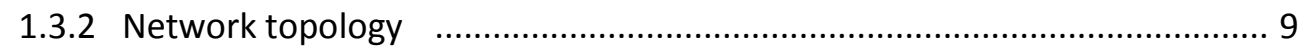

1.3.3 Network operation …..................................................................... 11

1.3.4 Network optimal management …........................................................ 12

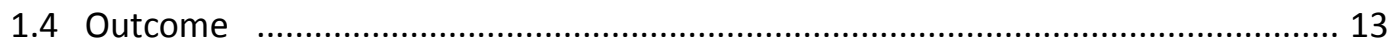

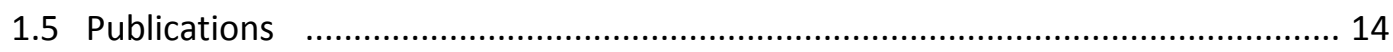

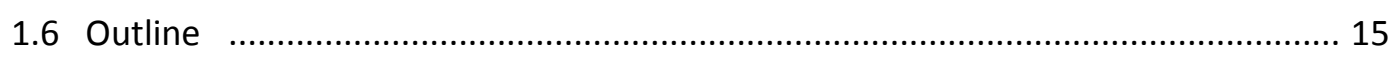

2. Simplified Modelling and Validation of an Industrial Diesel HDS Plant .......... 17

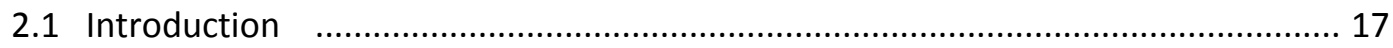

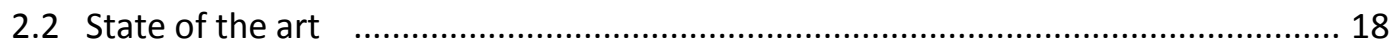

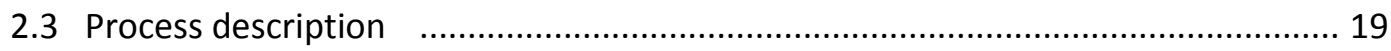

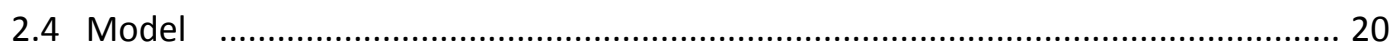

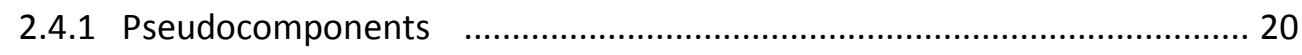

2.4.2 Material balances …...................................................................... 21

2.4.3 Momentum balances ……………………..................................... 21

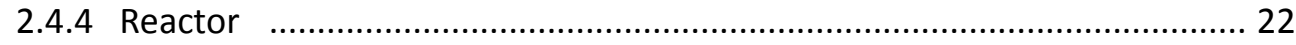

2.4.5 High pressure separator …................................................................. 24

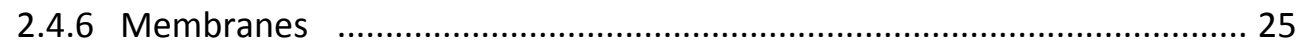




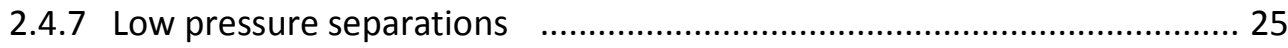

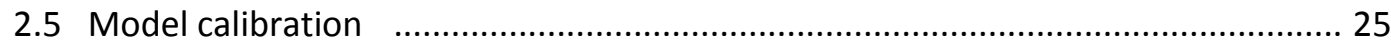

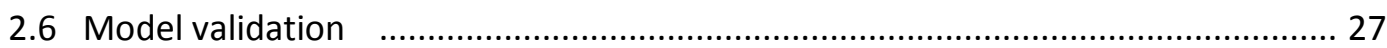

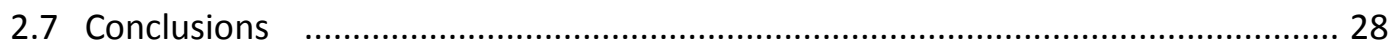

3. Self-Optimizing Control for $\mathrm{H}_{2}$ Optimization in an Industrial HDS Plant $\ldots . . . . . .29$

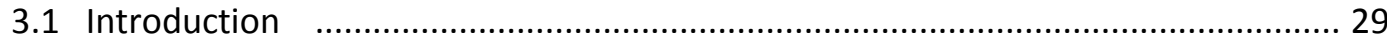

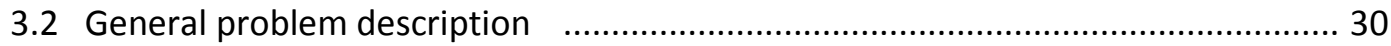

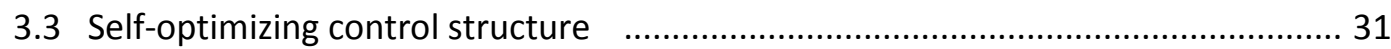

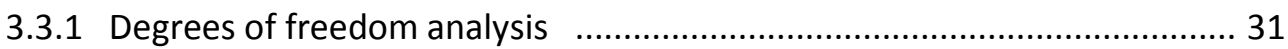

3.3.2 Definition of optimal operation: cost and constraints f............................ 31

3.3.3 Identification of important disturbances ……….................................. 32

3.3.4 Regions of active constraints (modes of operation) ….............................. 33

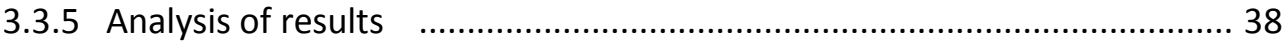

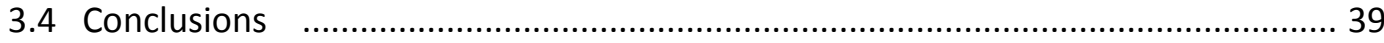

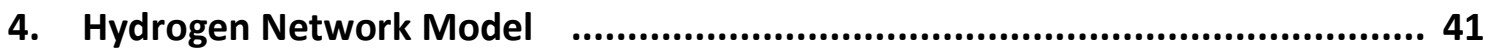

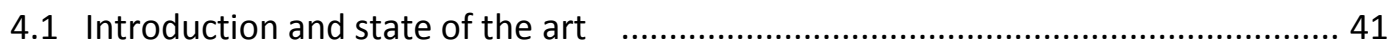

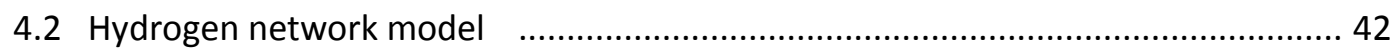

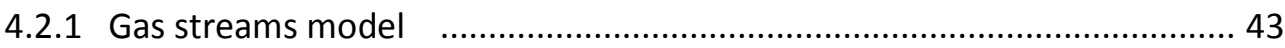

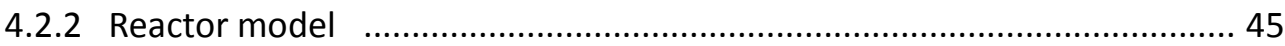

4.2.3 Membranes model ........................................................................... 49

4.2.4 Separator model $\quad$.............................................................................. 50

4.2.5 Low-pressure separator model ………….......................................... 55

4.2.6 Model of compressors, mixers, splitters, valves, pipes, meters …........... 55

4.2.7 Model sensitivity for a consumer plant ……….................................... 61

4.3 Model implementation in EcosimPro ${ }^{\circledR}$ environment …......................................... 68

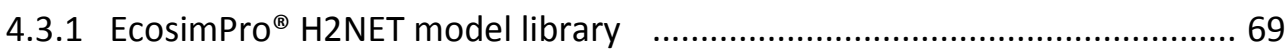

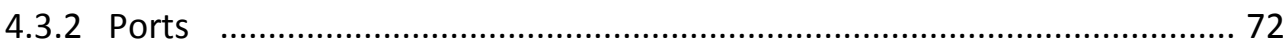

4.3.3 Selection of boundary variables for simulation …..................................... 84

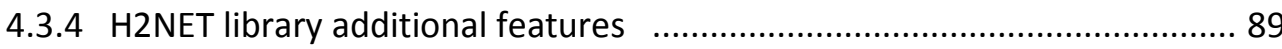

4.3.5 Configuration, data acquisition and simulation …................................. 91

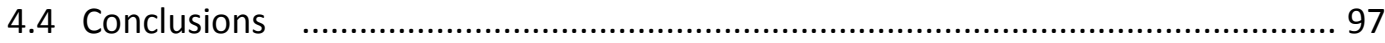


5. Hydrogen Network Data Reconciliation $\quad$................................................ 101

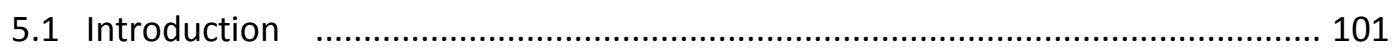

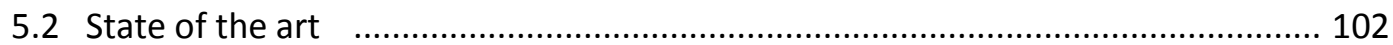

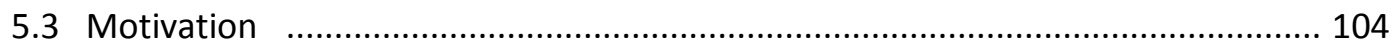

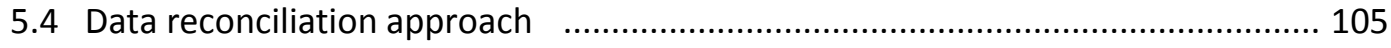

5.4.1 Systematic errors in practice …………............................................ 105

5.4.2 Data reconciliation formulation ........................................................... 108

5.4.3 Data reconciliation degrees of freedom and constraints ..................... 112

5.5 Data reconciliation implementation ……….................................................. 118

5.5.1 Linear constraints management …....................................................... 119

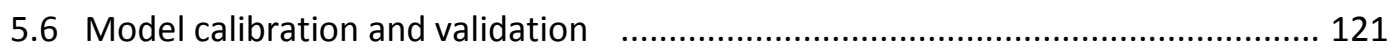

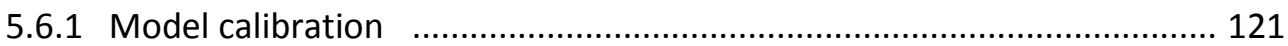

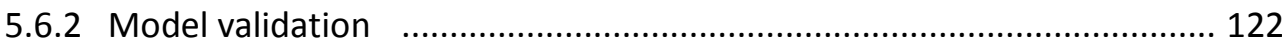

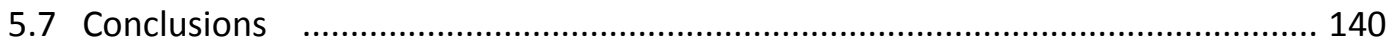

6. Hydrogen Network Optimal Redistribution $\quad$................................................ 143

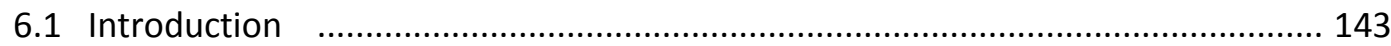

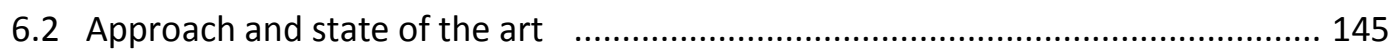

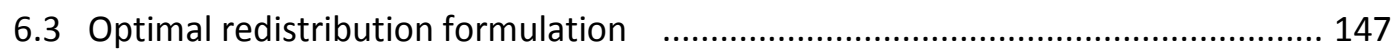

6.3.1 Optimal operation formulation: cost function …................................. 147

6.3.2 Optimal operation formulation: decision variables and constraints ...... 150

6.4 Optimal redistribution implementation ………............................................... 156

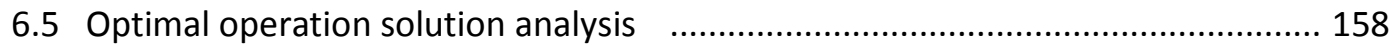

6.5.1 Description of the general framework for optimal management …...... 158

6.5.2 Optimal operation solution analysis …............................................... 161

6.5.3 Case study conclusions …............................................................... 163

6.6 Additional exploitation of model and optimization capabilities .......................... 164

6.6.1 Study on design alternatives regarding new connections ..................... 165

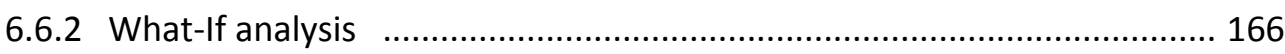

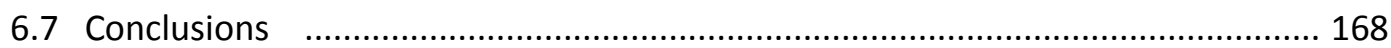

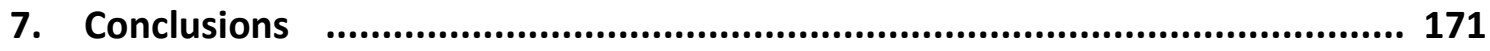

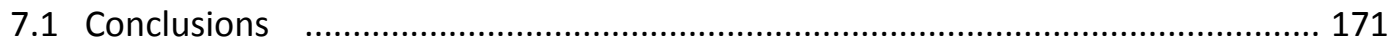

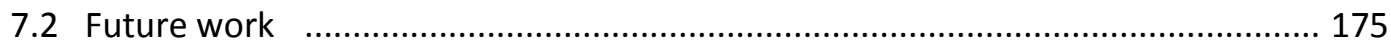




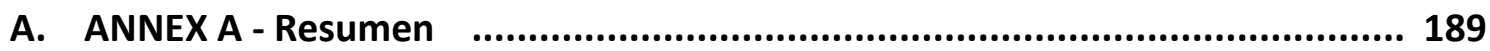

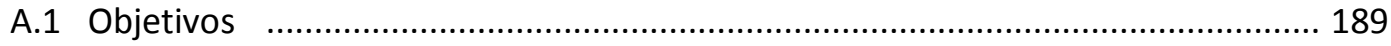

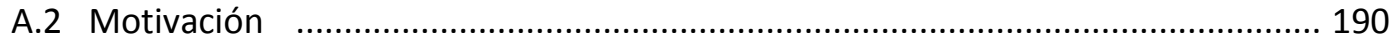

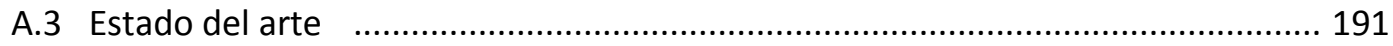

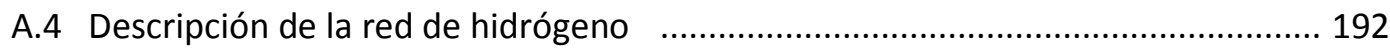

A.4.1 Descripción de una unidad hidrodesulfuradora HDS general ................ 193

A.4.2 Gestión óptima de la red de hidrógeno .................................................. 194

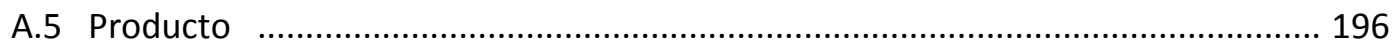

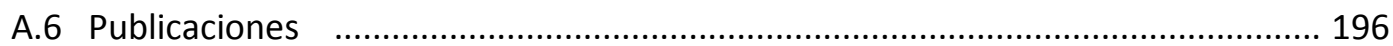

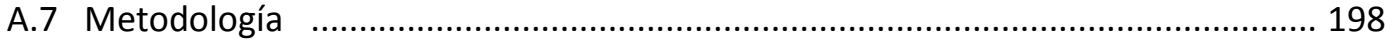

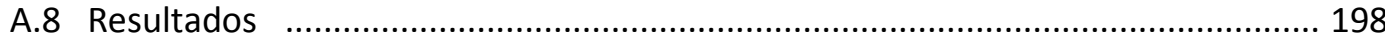

A.8.1 Modelo simplificado y validación de una HDS industrial …................... 198

A.8.2 Control self-optimizing para la gestión de $\mathrm{H}_{2}$ aplicado a HDS …............ 203

A.8.3 Modelo de la red de hidrógeno …………............................................ 205

A.8.4 Reconciliación de datos de la red de hidrógeno .................................... 210

A.8.5 Redistribución óptima de la red de hidrógeno ...................................... 216

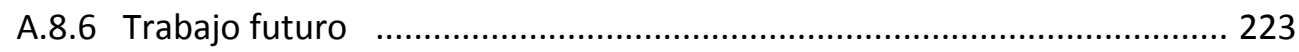




\section{SUMMARY}

A study on the optimal real-time management of hydrogen $\mathrm{H}_{2}$ networks in oil refineries has been carried out, with reference to the Petronor ${ }^{\circledR}$ oil refinery, belonging to Repsol ${ }^{\circledR}$ group and located in Muskiz (Vizcaya). The thesis work is an application of well-known and established techniques as process modeling and optimization to a currently interesting subject, $\mathrm{H}_{2}$ networks in oil refineries. Coherent and robust results have been achieved, and the solution is ready to be applied in the industrial practice.

Firstly, a simplified dynamic model of an industrial diesel hydrodesulphurization plant, one of the most important $\mathrm{H}_{2}$ consumer plants in the network, was developed with the aim of gaining insight into process operation, influencing inputs and parameters, as well as variable sensitivities. The model is based on first-principles balances and constitutive equations, combined with black-box neural networks to model the kinetic coefficients for the reactions proposed. The feasibility of the approach was proved; despite the lack of on-line measurements for feedstock composition and sulfur content, model predictions for $\mathrm{H}_{2}$ consumption resulted even better than expected. Additionally, the developed model was used to study the implementation of optimal policies as control strategies according to the selfoptimizing technique (Skogestad, 2000), again with the purpose of $\mathrm{H}_{2}$ optimal management but only considering the aforementioned consumer plant isolated from the whole network. The resulting control structure is simple, easy to implement (feedback control with PI controllers) and assures the global optimum in nearly all cases, although an upper RTO layer will be needed to guarantee the operation in the adequate region, with not frequent updates. Only in one scenario, very uncommon, a trade-off arises regarding the unconstrained degrees of freedom, and a self-optimizing control variable must be looked for to assure close to optimal operation avoiding more complex on-line optimization techniques.

Secondly, a real-time optimization RTO approach was followed for the purpose of real-time optimal operation of the global refinery $\mathrm{H}_{2}$ network. In order to estimate the plant state while taking advantage of redundancy in measurements, a data reconciliation step is performed previously to the RTO. Both problems are solved by optimization techniques, by minimization of two defined cost functions, based on the same process model and subject to certain process constraints in each case. A simplified model based on first principles has been proposed for the network aimed at the optimal $\mathrm{H}_{2}$ management; model complexity corresponds with the availability of on-line measurements and the model aims; parameters were limited according 
to the normal operating ranges and the sensitivities for important variables were analyzed. Modeling assumptions are justified based on historical data from laboratory quality measurements and the contribution of the different terms to the total $\mathrm{H}_{2}$ production. Off-line validation has been performed, and the model robustness and flexibility verified.

The data reconciliation problem for an accurate plant state estimation is a challenging problem due to uncertainty, which is caused by several reasons; the main uncertainties regarding data reconciliation were identified and dealt with. Practical implementation problems have also been tackled, in particular the automatic detection of wrong measurements with simple rules based on the measured standard deviations, as well as the management of linear constraints to guarantee model convergence in the search region. The data reconciliation results were validated off-line according to trends in raw measurements and valve openings, in addition to process knowledge.

Regarding the optimal $\mathrm{H}_{2}$ redistribution, solutions could be easily parameterized corresponding to the logical optimal operation; trade-offs were identified in certain cases, although the margin in those cases was not significant. An analysis of solutions showed that: a) regarding high pressure (HP) purges, the solution is the logical one, that is, to purge Low Purity Header $\mathrm{LPH}$ excess, if any, at the network scope through the HP purge at lower $\mathrm{H}_{2}$ purity until it gets saturated, following an increasing order of $\mathrm{H}_{2}$ purity to purge in consumer plants; b) regarding trade-offs arising in redistribution from producer plants (high purity, expensive) and LPH (low purity, cheap) to consumer plants, there is margin for profit although small; nevertheless the RTO approach can prove advantageous due to frequent changes in scenarios, aiding the operators to save time in the identification and implementation of the optimal policy. Furthermore, the analysis can also be valuable to reveal economic-technical trade-offs, where non-linear behaviours arise.

The optimal operation of $\mathrm{H}_{2}$ networks in oil refineries has already been addressed from a design perspective by other research groups, in particular by the Manchester University with the pinch technology. To the best of my knowledge, reactor model accounts for the same phenomena; however although more rigorous and accurate models for the thermodynamic equilibrium relations are used in this case, plant model flexibility is reduced according to the design purpose, i.e. operating conditions are fixed regarding reactor inlet and outlet $\mathrm{H}_{2}$ purities, in such a way that equilibrium relations in the separators hold. Very recently, the subject has also been addressed from an on-line operational viewpoint by companies providing services, like Inprocess $^{\circledR}$ based in Barcelona that takes advantage of Hysys ${ }^{\circledR}$ commercially available process simulator and its optimization capabilities to determine the optimal operation. To the best of my knowledge, although the same rigorous and systematic approach is shared regarding optimization techniques, important assumptions and process constraints, nevertheless a flexible and easily updated model calibration is worthwhile, which can be enhanced with an intended model as the one developed.

The $\mathrm{H}_{2}$ network simulation is available in the EcosimPro ${ }^{\circledR}$ modeling environment, as well as the implementations for the two optimizations problems to solve the data reconciliation and the optimal redistribution, using Snopt ${ }^{\circledR}$ as NLP solver based on a SQP algorithm. A library with components modeling each of the units of the $\mathrm{H}_{2}$ network has also been developed in the 
EcosimPro ${ }^{\circledR}$ environment, together with functions for the automatic generation of the code needed to implement both optimization problems. 



\section{CHAPTER 1}

\section{INTRODUCTION}

A study on the optimal real-time management of hydrogen $\mathrm{H}_{2}$ networks in oil refineries has been carried out, with reference to the Petronor ${ }^{\circledR}$ oil refinery, belonging to Repsol ${ }^{\circledR}$ group and located in Muskiz (Vizcaya). The thesis work is an application of well-known and established techniques as process modeling and optimization to a currently interesting subject, $\mathrm{H}_{2}$ networks in oil refineries. Coherent and robust results have been achieved, and the solution is ready to be applied in the industrial practice.

A problem of industrial significance has been addressed by means of modeling, optimization and advanced control techniques, with the general purpose of process operation improvement, resource (material and/or energy) saving, and process knowledge enhancement. Modeling libraries in EcosimPro ${ }^{\circledR}$ have been also developed.

\section{1- MOTIVATION}

Petroleum refineries process fossil feedstock to produce fuel for heating and transport purposes, as well as precursors for the petrochemical industry, requiring large amounts of raw materials and energy. Resource saving is a major concern for process plants in general, and in particular for oil refineries due to the low profit margins and big quantities involved. Efficiency in the use of hydrogen as a raw material in the oil refinery of Petronor ${ }^{\circledR}$ (Muskiz, Vizcaya), belonging to Repsol ${ }^{\circledR}$ group, is considered. Hydrogen $\mathrm{H}_{2}$ is an expensive utility required in many processes in an oil refinery, which is distributed by means of a $\mathrm{H}_{2}$ network from producer to consumer plants. In consumer plants, $\mathrm{H}_{2}$ is mainly used as reactant for desulfurization, denitrification and de-aromatization of naphtha and diesel, in the presence of other $\mathrm{H}_{2}$ consuming side reactions. Desulfurization and de-nitrification reactions enable not to generate acid gases $\left(\mathrm{SO}_{\mathrm{x}}, \mathrm{NO}_{\mathrm{x}}\right)$ either when used as heating fuel or in combustion engines, thus avoiding atmosphere pollution. 


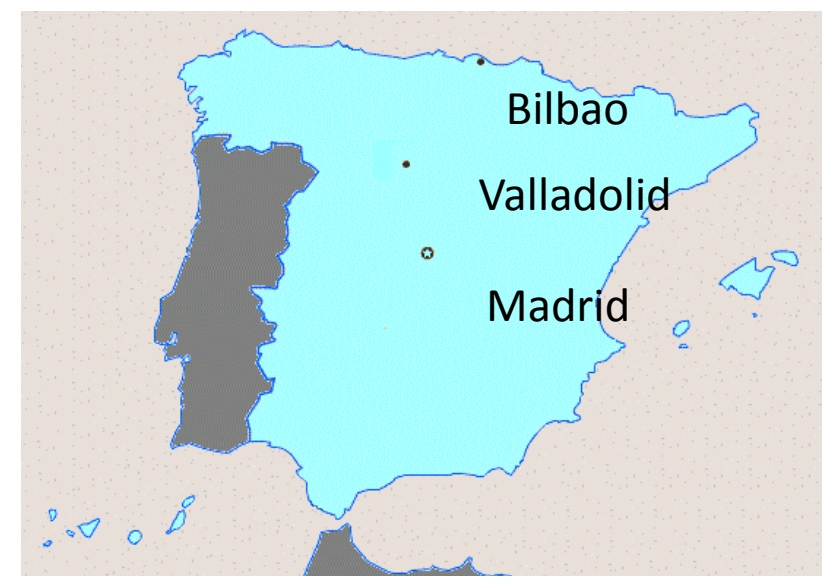

Figure 1.1. Petronor ${ }^{\circledR}$ refinery in Muskiz is $20 \mathrm{~km}$ away from Bilbao (Vizcaya).

In recent years when heavier fuels are being processed, and also due to more strict environmental regulations, $\mathrm{H}_{2}$ requirements have experienced a steady increase, with $\mathrm{H}_{2}$ gaining significant importance in the refinery global economic balance. An efficient use of $\mathrm{H}_{2}$ in the daily operation is desired not only for its high production cost, but also because the economic penalty is even higher in scenarios where $\mathrm{H}_{2}$ production capacity is bottleneck for oil processing capacity. Furthermore, decisions regarding $\mathrm{H}_{2}$ management are complex as many plants and operating constraints are involved in the network operation with a high degree of interrelation, not only from an optimality viewpoint but also from a practical viewpoint because several operators at different control rooms are typically in charge; thus a quantitative criterion for decision support can prove very valuable. In addition, feedstock usually changes every two-three days, and also certain product specifications can vary according to the global management of the refinery; as a consequence scenarios regarding $\mathrm{H}_{2}$ consumption in individual consumer plants can experience frequent significant changes, therefore a tool intended for real-time decision-making purposes regarding optimal operation is interesting.

The approach to deal with the $\mathrm{H}_{2}$ network optimal management is driven by an operational framework where $\mathrm{H}_{2}$ production must always exceed $\mathrm{H}_{2}$ consumption, because $\mathrm{H}_{2}$ deficit is extremely damaging for catalysts being catalysts very expensive. As $\mathrm{H}_{2}$ accumulation in a buffer vessel is not possible, besides the slow dynamic for $\mathrm{H}_{2}$ production in the furnaces of the intended steam-reforming plants, the target of $\mathrm{H}_{2}$ production minimization has to be achieved by means of:

- a good dynamic fit of $\mathrm{H}_{2}$ production to consumption, in order to minimize excess sent to the Fuel Gas network under pressure control in the headers. This is a controllability problem, addressed with a MPC designed and commissioned by the Petronor ${ }^{\circledR}$ Advanced Control Department, in cooperation with the Production Department. The purpose is to control certain determining specifications, therefore avoiding/reducing the gaps providing for buffer margin in case of disturbances; the MPC accounts for the most significant interrelations in the 
multivariable control of the LPH low-purity header, where excess from consumers is poured and which serves as make-up for certain consumer plants.

- a better $\mathrm{H}_{2}$ redistribution from $\mathrm{H}_{2}$ producer to $\mathrm{H}_{2}$ consumer plants. This problem is of a different nature, acting on a slower time-scale in an upper layer in the hierarchy than the aforementioned advanced regulatory control (MPC), and is addressed with an RTO approach. The decisions to be taken in the $\mathrm{H}_{2}$ network optimal management are:

- which plants must produce $\mathrm{H}_{2}$ and their production rates;

- which combination of make-up flows from each header must provide $\mathrm{H}_{2}$ to each consumer plant.

This is the problem tackled in this work. A simple model has been proposed, assumptions have been justified, and the actual network state has been obtained by solving a data reconciliation problem with optimization techniques, taking advantage of all the available redundant on-line measurements.

\section{2- STATE OF THE ART}

The optimal operation of $\mathrm{H}_{2}$ networks in petroleum refineries has already been addressed by other research groups from different perspectives:

- Design.

The research group at the University of Manchester has been working on the topic for years, developing the pinch technique for $\mathrm{H}_{2}$ networks analogous to the pinch technique for heat exchanger networks.

To the best of my knowledge, reactor model accounts for the same phenomena as the one proposed in this work; however although more rigorous and accurate models for the thermodynamic equilibrium relations are used in the Manchester tool, plant model flexibility is reduced according to the design purpose: the $\mathrm{H}_{2}$ purity from all the producers and the $\mathrm{H}_{2}$ purity of off-gases from all the consumers and purifiers are considered as constant, i.e. operating conditions are fixed regarding reactor inlet and outlet $\mathrm{H}_{2}$ purities, in such a way that equilibrium relations in the separators hold. Therefore, real-time process variability is not considered in this approach aimed for design purposes.

- On-line optimal operation.

Certain companies providing services, like Inprocess ${ }^{\circledR}$ located in Barcelona, offer a Hysys ${ }^{\circledR}$ based simulation package that takes advantage of Hysys ${ }^{\circledR}$ commercially available process simulator and its optimization capabilities to determine the optimal operation of the $\mathrm{H}_{2}$ network.

To the best of my knowledge, although the same rigorous and systematic approach is shared regarding optimization techniques, important assumptions and process 
constraints, nevertheless a flexible and easily updated model calibration is worthwhile, which can be enhanced with an intended model as the one developed in this work.

The theoretical framework supporting this work is well established both in the scope of process modeling based on first-principles and in the scope of optimization with different purposes, mainly process operation improvement. Optimization techniques are employed in the process industry in different fields: design, predictive control for multivariable process operations, scheduling. In the oil refining business, big-size LP problems are daily solved to decide on production according to current prices for crude-oil and products. Also medium-size LP problems are solved on-line in MPC (model predictive control) applications, especially in distillation columns due to their high contribution to the total energy consumption together with the difficulty of the multivariable control problem in these units. The use of deterministic optimization algorithms, as the SQP employed, is also widespread.

Different authors have written about economic perspectives; Forsberg (2005) deals with the global economy regarding $\mathrm{H}_{2}$ and its use as energy intermediate and its role in improving fuel quality and environmental requirements; Bartels et al. (2010) show that the most economical sources of hydrogen are coal and natural gas, although alternative energy sources may become more economical in the future.

In a scenario with increasing demands for $\mathrm{H}_{2}$, the management and optimization of the $\mathrm{H}_{2}$ system is becoming very important for the refinery optimum economics as a whole, which has been reflected in many works in the past years mainly from a design perspective.

Towler et al. (1996) introduced a graphical method to analyze hydrogen network using value composite curves. Alves and Towler (2002) proposed the hydrogen pinch analysis for targeting the minimum $\mathrm{H}_{2}$ consumption of the whole $\mathrm{H}_{2}$ system, developing a framework of sinks and sources similar to the pinch analysis for heat exchanger networks. The graphical based approaches consider solely the purity and flowrate of streams, but the pressure of hydrogen suppliers and hydrogen consumers is not considered. Hallale and Liu (2001) first developed an improved superstructure optimization method for $\mathrm{H}_{2}$ network accounting for the pressure constraints as well as compressors for retrofit scenarios, determining the minimum utility consumption and the maximum recovery of the $\mathrm{H}_{2}$ network. Fonseca et al. (2008) employed the LP model to optimize a $\mathrm{H}_{2}$ network refinery; Liu and Zhang (2004) provided a detailed model of purification units for selection of purification processes and their integration in $\mathrm{H}_{2}$ networks. Liao et al. (2010) considered purification processes and relations for compressors for refinery $\mathrm{H}_{2}$ management and demonstrated the application of superstructure based approach with a MINLP model for retrofit design of an existing refinery, developing a systematic approach that provides more network structure possibilities for the placement of compressors and purifiers. Ahmad et al. (2010) developed a novel approach for the design of flexible $\mathrm{H}_{2}$ networks that can remain optimally operable under multiple periods of operation. El-Halwagi et al. () used graphical methods and mass exchanger networks to minimize fresh sources, by using segregation, mixing, and direct recycle/reuse strategies. Khajehpour et al. (2009) proposed objective functions that minimize waste flows containing $\mathrm{H}_{2}$ and reduce $\mathrm{H}_{2}$ 
production, with reduced superstructure based on experience and engineering judgment. Kumar et al. (2010) noted that the effect of the cost parameter on optimum distribution of $\mathrm{H}_{2}$ is the most important study parameter. They considered the ability of the MINLP model to evaluate many complexities of a real refinery system in two case studies. The LP, NLP, MILP and MINLP models were developed and the characteristics of these models were analyzed.

Jiao et al. (2011a) proposes a novel sequential two-step method to optimize the $\mathrm{H}_{2}$ distribution network, where the optimization process can be divided into two steps based on the structure characteristics of the $\mathrm{H}_{2}$ network. The first step is to optimize the $\mathrm{H}_{2}$ purification network whose objective is to maximize the value created by purifiers and the minimization of operation cost and annualized capital cost. The second step is to optimize the $\mathrm{H}_{2}$ supply network whose objective is to minimize the total annual cost which includes operation cost and annualized capital cost. Jiao, Su et al. (2012) make the proposed approaches more suitable for real systems, considering as variables the flowrate and purity at the reactor inlet of $\mathrm{H}_{2}$ consumers and the $\mathrm{H}_{2}$ recovery of purification units, with a constraint for the minimum pure hydrogen of $\mathrm{H}_{2}$ consumers that must be satisfied. They also propose other method to retrofit the $\mathrm{H}_{2}$ network, based on a simultaneous optimization approach which can optimize $\mathrm{H}_{2}$ purification network and $\mathrm{H}_{2}$ supply network simultaneously and the objective is also to minimize total annual cost, with a MILP linearization technique for the MINLP model. Jiao et al. (2012) consider uncertainties in the optimization approach for the optimization of $\mathrm{H}_{2}$ network. A novel chance constrained programming approach is proposed. The stochastic properties of the uncertainties are explicitly considered, and a suitable compensation between the profit and the probability of constraints violation is achieved.

Sardashti et al. (2011) deal with the recovery of $\mathrm{H}_{2}$ from refinery off-gases by purifiers. The cost of this process can be significantly lower than for buying or producing $\mathrm{H}_{2}$. The change of natural gas feedstocks to off-gas in the steam reformer enables a great decrease of the total annual cost. In most cases, the purity of $\mathrm{H}_{2}$ in off-gas streams is not adequately high to be recycled. Therefore, it decreases the efficiency of the purifiers. Off-gases can be used for feedstocks of a steam reformer in addition to the fuel system. $\mathrm{CO}_{2}$ is the other generated steam in reforming processes which is mentioned as a green-house gas. By using this methodology, $\mathrm{CO}_{2}$ emission in $\mathrm{H}_{2}$ networks can be constrained. Other studies have been performed on the management of hydrogen networks with purifiers and hydrogen recovery from off-gases, see for example Agarwal, Biegler et al. (2009), Hallale and Liu (2001), Liao et al. (2010). Peramanu et al. (1999) evaluated the economic performance of different purification processes for off-gases.

Other authors have focused on the planning and scheduling of the hydrogen networks, like Van den Heever and Grossmann (2003); Zhang, Zhu and Towler (2001) present a method for overall refinery optimization through integration of the hydrogen network and the utility system with the material processing system. A new optimization method is proposed that is developed on the basis of a sound understanding of interactions between the three systems and the proper use of mathematical modeling. This method considers the optimization of refinery liquid flows, hydrogen flows, and steam and power flows simultaneously. As a result, this method furnishes new insights into the problem of refinery optimization and exploiting the true potential of the processes. 
Also from a topological design viewpoint, Luckwal and Kumar (2009) proposes the use of an offsite storage facility for $\mathrm{H}_{2}$, consisting of bullets and a compressor to top off the bullets, used to supply $\mathrm{H}_{2}$ to generation units during emergencies, startups and shutdowns. This provides a buffer volume to supply $\mathrm{H}_{2}$ to the network during sudden pressure dips, and recover most of the spillover by routing it to the bullet via the compressor.

From a real-time operational point of view, different recent works result from the interest in the on-line optimal operation of $\mathrm{H}_{2}$ networks. Lee, Goodhart et al. (2008) describes the typical operational framework to ensure steady $\mathrm{H}_{2}$ network pressure: when insufficient $\mathrm{H}_{2}$ is available, production rates will fall, having significant financial penalties on the refinery operation. If excess $\mathrm{H}_{2}$ is produced then it must be downgraded to fuel or potentially flared, having a negative impact both economic and environmental. The target is to balance $\mathrm{H}_{2}$ production and distribution in the refinery to match rapidly changing demands, which provides significant benefits in terms of cost of operation. The work deals with header pressure control, proposes a controller scheme modification to break the dynamic response within headers and includes various feed-forward functions to improve disturbance rejection, stabilization and control performance in important variables. Similarly, Mastrogiacomo et al. (1997) report energy savings by implementing advanced control of the hydrogen plant and hydrogen grid at its Montreal refinery, maximizing $\mathrm{H}_{2}$ recovery.

\section{3- HYDROGEN NETWORK DESCRIPTION}

A petroleum refinery includes many unit operations. Hydrogen $\mathrm{H}_{2}$ is an expensive utility required in many processes in an oil refinery, which is distributed by means of a $\mathrm{H}_{2}$ network from producer to consumer plants.

Most of the consumer plants are hydrotreaters, where $\mathrm{H}_{2}$ is mainly used as reactant for desulphurization, de-nitrification and de-aromatization of naphtha and diesel, in the presence of other $\mathrm{H}_{2}$ consuming side reactions. The removal of sulphur-containing and nitrogencontaining hydrocarbons in catalytic hydrodesulphurizers, converted to hydrogen sulfide and ammonia, enable not to generate acid gases $\left(\mathrm{SO}_{\mathrm{x}}, \mathrm{NO}_{\mathrm{x}}\right)$ either when used as heating fuel or in combustion engines, thus avoiding atmosphere pollution.

Another important $\mathrm{H}_{2}$ consuming process is hydrocracking, which is a catalytic cracking where $\mathrm{H}_{2}$ is used to break C-C bonds unlike a hydrotreater, where $\mathrm{H}_{2}$ is used to cleave C-S and C-N bonds. It is a process common in Europe and Asia because those regions have high demand for diesel and kerosene. The products from hydrocracking are diesel and jet fuel, although low sulphur naphtha fractions and LPG are also produced; all these products have a very low content of sulphur and other contaminants, because sulphur and nitrogen compounds present in the feedstock are converted to hydrogen sulfide and ammonia. Hydrocarbon chains are broken and rearranged and $\mathrm{H}_{2}$ is added, converting aromatics and olefins into naphthenes and alkanes; in general saturated hydrocarbons are obtained, ranging from ethane, LPG to heavier hydrocarbons consisting mostly of isoparaffins. 
Regarding producer plants, high purity $\mathrm{H}_{2}$ is produced in steam-reforming furnaces, named $\mathrm{H} 3$ and $\mathrm{H} 4$ in this case ( $\mathrm{H} 3:>0.90 \% 1 \mathrm{~mol}, \mathrm{H} 4: 0.999 \% 1 \mathrm{~mol})$. Steam reforming of natural gas is the most common method of producing bulk hydrogen. The reaction, where steam reacts with methane to yield carbon monoxide and hydrogen, is conducted at high temperatures around $700-1100 \stackrel{\circ}{ } \mathrm{C}$ and in the presence of a metal-based catalyst (nickel). Additional $\mathrm{H}_{2}$ can be recovered by a lower-temperature gas-shift reaction with the carbon monoxide produced, being converted into carbon dioxide.

Another two low-purity $\mathrm{H}_{2}$ producer plants named P1 and P2 ( 0.75\%1 mol) exist, not being truly decision variables in operation as $\mathrm{H}_{2}$ is a byproduct of the catalytic reforming process. In catalytic reforming processes, naphthas distilled from crude oil typically having low octane ratings are converted into more complex molecules with a higher octane rating value: lowoctane linear hydrocarbons (paraffins) are converted into branched alkanes (isoparaffins) and cyclic naphthenes, which are then partially dehydrogenated to produce high-octane aromatic hydrocarbons. The dehydrogenation also produces significant amounts of byproduct hydrogen gas, which is fed into other refinery processes. As compared to the $\mathrm{H}_{2}$ produced in steamreforming furnaces, this is a low-purity $\mathrm{H}_{2}$ ranging from 0.65 to $0.83 \% 1 \mathrm{~mol}$, because hydrogenolysis is a side reaction which produces light hydrocarbons of lower value accompanying the $\mathrm{H}_{2}$, such as methane, ethane, propane and butanes. Hydrotreatment of the feedstock is conducted in other hydrodesulphurizer plants prior to the catalytic reforming, in order to protect the catalysts.

The two steam-reforming furnace producer plants are shown in red in Fig. 1.2, with their corresponding headers for $\mathrm{H}_{2}$ distribution in red and pink respectively. Catalytic-reforming producer plants are shown in purple, with linked headers in purple too. And hydrodesulfurization consumer plants appear in green, with make-up streams from different headers.

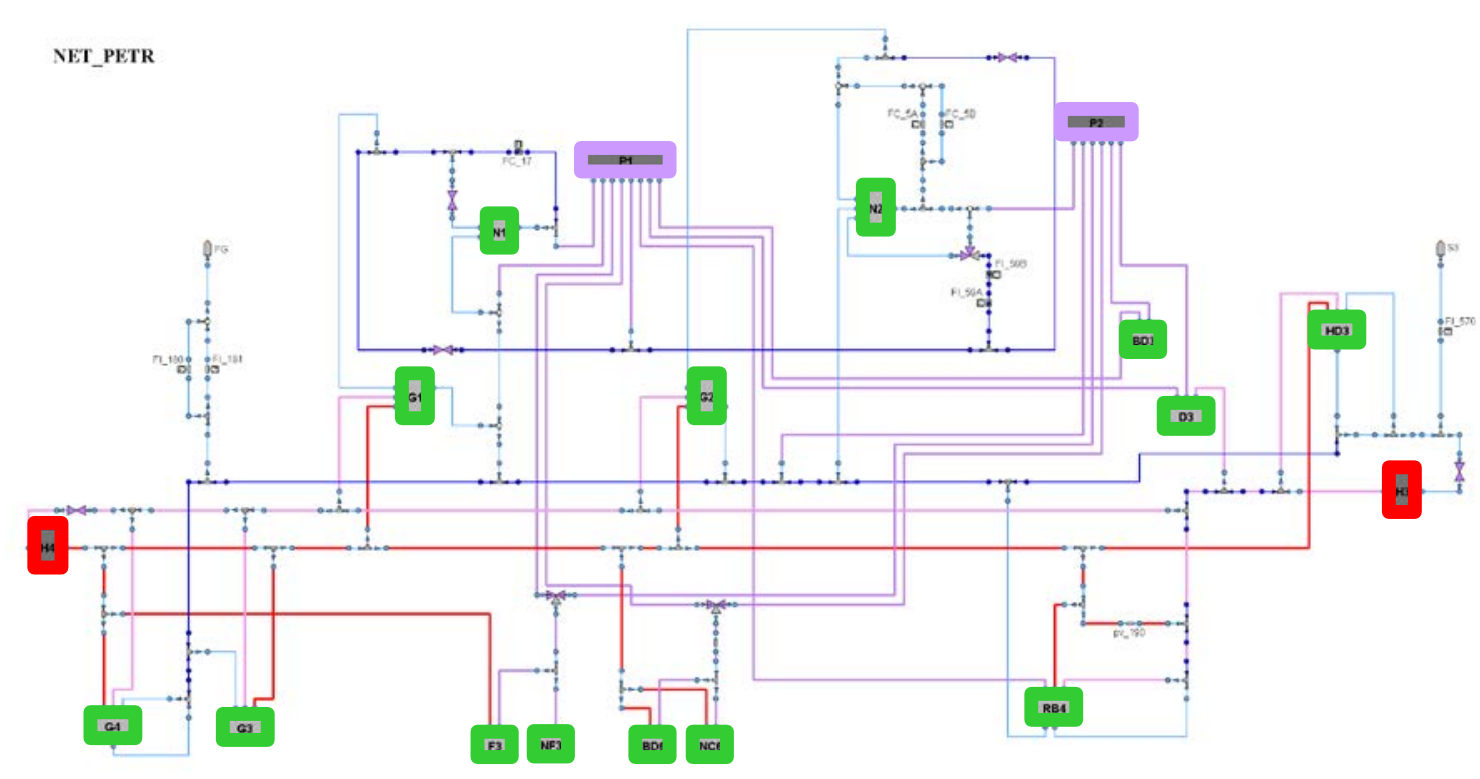

Fig. 1.2. Schematic of the Petronor ${ }^{\circledR}$ refinery $\mathrm{H}_{2}$ network. 


\subsection{1- General hydrodesulphurizer HDS plant description}

In a general consumer plant (Fig. 1.3), before entering the reactor $(\triangle R)$ the hydrocarbon $(H C)$ feed is mixed with the recycled $\mathrm{H}_{2}$ stream $(R)$ and with a make-up $\mathrm{H}_{2}$ stream from the network: typically from the two producer plants $\mathrm{H} 3$ and $\mathrm{H} 4$, and from one Low Purity distribution Header LPH collecting excess from other consumer plants and catalytic reforming units. After being separated in a high pressure HP separation drum (HPsep), non-reacted $\mathrm{H}_{2}$ is partially recycled $(R)$, and can be partially purged to another Low Purity distribution Header $\left(L P H_{H P}\right)$. Most of the gas from the HP separator is recycled into the reactor inlet. A HP purge is usually needed to maintain the $\mathrm{H}_{2}$ purity minimum constraint in the HP system, while avoiding the accumulation of light ends in the system. Light ends are both generated in the reactor and supplied in the make-up due to low-purity $\mathrm{H}_{2}$ sources.

Reacted sulphur turns into hydrogen sulfide $\mathrm{H}_{2} \mathrm{~S}$, which is removed by absorption on an amine solution. Downstream of the high pressure HP separator, several separators and distillation columns at lower pressure enable the complete separation of $\mathrm{H}_{2}$ and light gases from the desulfurized hydrocarbon; these streams from the medium and low pressure MP/LP separation processes (LPoper) are burnt as fuel gas $\left(F G_{L P}\right)$, since their $\mathrm{H}_{2}$ purity is not high enough for making profitable its recovery and reuse. Regarding losses from the MP/LP systems, these purges are not a degree of freedom in operation, being separated under pressure control with the purpose of the complete removal of gas from the liquid hydrocarbon processed. Losses from the HP system are of a different nature: these purges are generally manipulated or decision variables, and are usually sent to Low Purity Headers due to their high $\mathrm{H}_{2}$ purity, thus being reused in other consumer plants.

Whenever available, a membrane unit $(Z)$ makes possible to purify and recycle a permeate stream $\left(F_{P R M_{Z} Z}\right)$, at the expense of a purge to fuel gas $\left(F G_{Z}\right)$ with lower $\mathrm{H}_{2}$ purity.

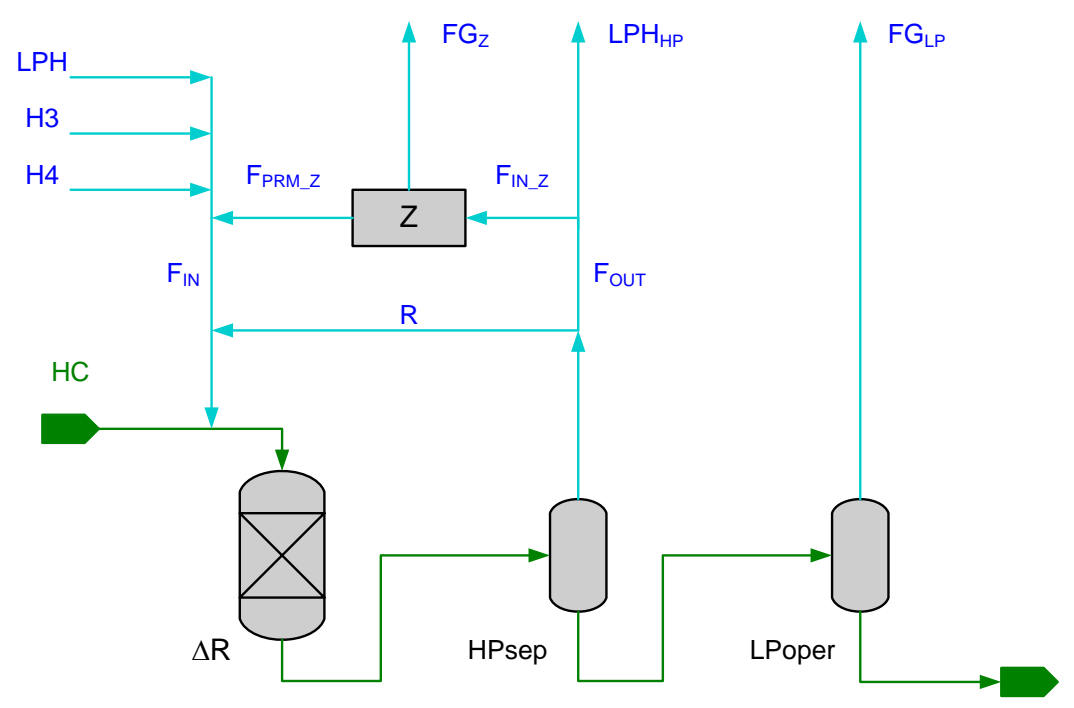

Fig. 1.3. Schematic of an $\mathrm{H}_{2}$ consumer plant with make-up from two Production Headers $(\mathrm{H} 3, \mathrm{H} 4)$ and from one Low Purity Header (LPH). 


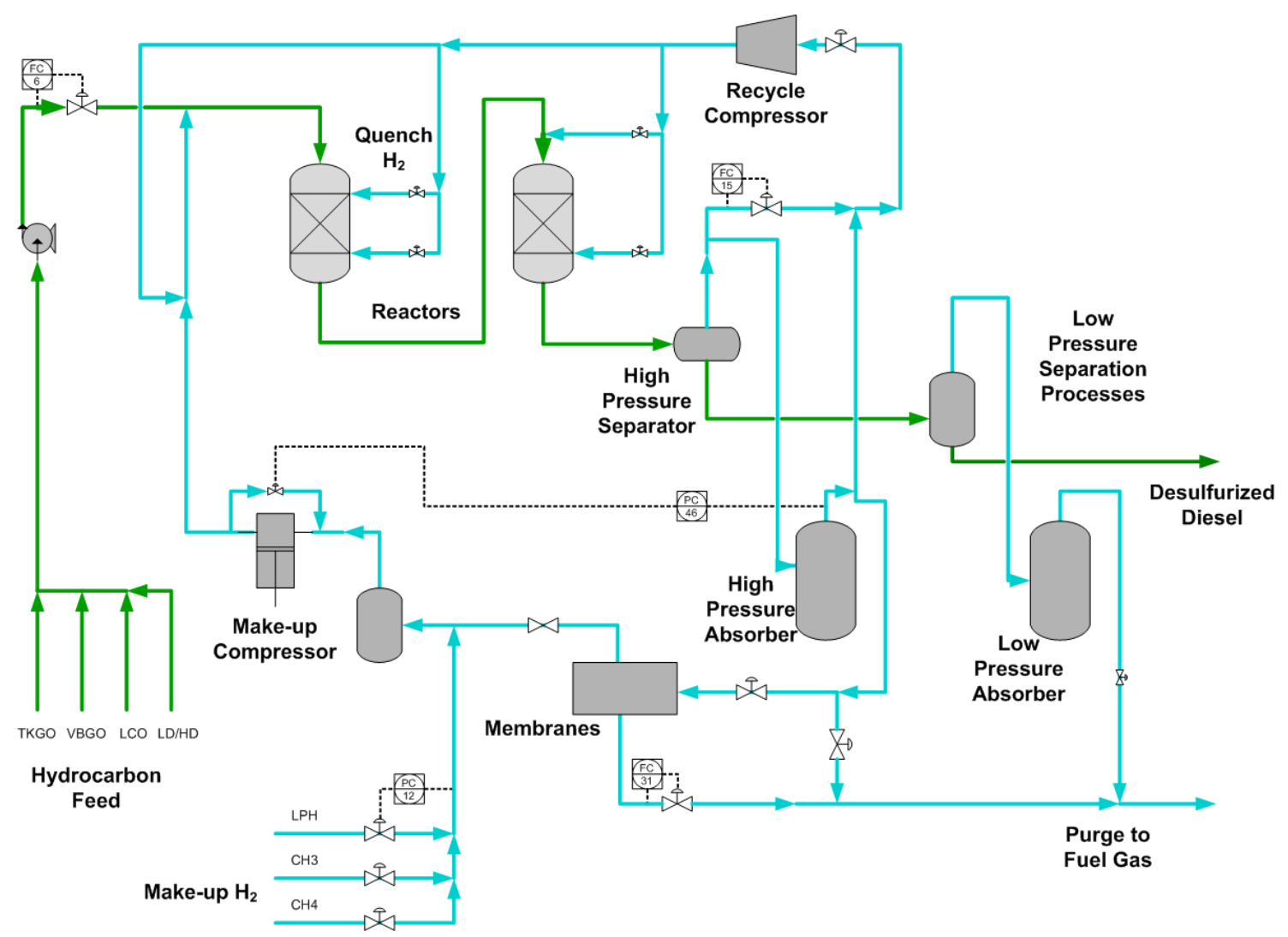

Figure 1.4. Simplified process flow diagram for a diesel hydrodesulfurizer plant with membranes.

A more detailed process flow diagram is shown in Fig. 1.4, with the different cuts of hydrocarbon mixed in the feed, the quench streams to the reactor, a bypass of the high pressure separator, and a direct HP purge to FG alternative to membranes.

\subsection{2- Network topology}

In order to make the most of the produced $\mathrm{H}_{2}$, high-pressure HP surplus from certain consumer plants is further used as make-up in other ones, in those cases where the HP purge $\mathrm{H}_{2}$ purity is high enough. A header named the low-purity header LPH exists where HP excess from those consumer plants operating at a significant $\mathrm{H}_{2}$ purity are poured, as well as part of the excess from the catalytic reforming units. Simultaneously, this LPH provides make-up for several important consumer plants.

Not all consumer plants are equally important regarding $\mathrm{H}_{2}$ requirements; moreover, they also differentiate for the make-up and purge headers available. A brief summary is contained in Table 1.1. The highest flexibility for make-up corresponds to the biggest consumer plants, which can be provided from three different headers, hereinafter referred to as $\mathrm{H} 4 \mathrm{H}, \mathrm{H} 3 \mathrm{H}$ and $\mathrm{LPH}$, corresponding respectively to producer plant $\mathrm{H} 4$, producer plant $\mathrm{H} 3$ and low-purity headers. 


\begin{tabular}{lcc}
\hline Plant & $\mathrm{H}_{2}$ Consumption & HP purges \\
\hline & & \\
BD3 & + & $\mathrm{FG}$ \\
BD6 & + & $\mathrm{FG}$ \\
D3 & & $\mathrm{FG}$ \\
SHU-F3 & ++ & $\mathrm{FG}$ \\
G1 & ++++ & $\mathrm{LPH}$ \\
G2 & ++ & $\mathrm{LPH} / \mathrm{Z}_{\mathrm{FG}}$ \\
G3 & ++++ & $\mathrm{ZFG} / \mathrm{FG}$ \\
G4 & ++++ & $\mathrm{LPH}$ \\
H3 & - & $-/ \mathrm{FG}$ \\
H4 & - & $-/ \mathrm{H} 3 \mathrm{H}$ \\
HD3 & +++++ & $\mathrm{LPH} / \mathrm{Z}_{\mathrm{FG}}$ \\
N1 & ++ & $\mathrm{G} 1 / \mathrm{LPH}$ \\
N2 & ++ & $\mathrm{G} 2 / \mathrm{LPH}$ \\
NC6 & +++ & $\mathrm{FG}$ \\
NF3 & ++ & $\mathrm{FG}$ \\
P1 & - & $\mathrm{LPH}$ \\
P2 & - & $\mathrm{LPH}$ \\
RB4 & & LPH \\
& & \\
\hline
\end{tabular}

Table 1.1. $\mathrm{H}_{2}$ consumption in plants and HP purges.

All consumer plants can be supplied from different alternative sources. Nevertheless, the flexibility in the global network operation is not high as many constraints and practical matters must be considered:

- Certain small consumer plants cannot be supplied from the LPH, because of the presence of hydrogen sulfide $\mathrm{SH}_{2}$ in the $\mathrm{LPH}$, and can only be provided directly from the catalytic reforming units.

- Certain plants do not have absorbers for $\mathrm{SH}_{2}$, therefore their HP purges to the LPH are very restricted to avoid contamination, as a fraction of the LPH flow rate is downgraded to FG serving as heating fuel. As a consequence, it is mandatory that these HP purges go to another hydrodesulphurizer plant downstream, which does have absorber and whose excess can be poured to the LPH for further use. Although more than one possibility exists to account for eventual plant shutdowns, only one alternative is preferential and commonly employed, not needing for neither manual valves nor shared connection lines thanks to a direct connection.

- Certain plants do not have a recycle stream, thus the minimum ratio $\mathrm{H}_{2} / \mathrm{HC}$ is achieved with the make-up stream, determining the path for of a significant amount of the produced $\mathrm{H}_{2}$. 
- Certain medium-size and small consumer plants are only supplied from one header. An alternative is also available accounting for eventual plant shutdowns, typically makeup from either P1 or P2 catalytic reforming units, however the exchange has to be done manually with non-automatic valves and requires operational effort which is only justified on occasions. In addition, other times the alternative make-up source requires shared connection lines, as a result being exclusive with other alternatives, or requiring additional labor and attention because of the connection of systems at different pressures.

For example, the compressors of the two catalytic reforming plants operate at different discharge pressures, therefore consumer plants are fed with either one or the other, but not simultaneously with both of them because this implies manual regulation of valve openings to assure good flow in the resulting scheme; it is feasible, although demanding in terms of labor. The same also applies in other cases of simultaneous make-up from different plants.

\subsection{3- Network operation}

In each consumer plant, more than one header is always available for pressure relief in all the three systems HP/MP/LP for security reasons, under a split-range pressure control structure. The different available headers for pressure relief follow the logical sequence for $\mathrm{H}_{2}$ use and exploitation, that is, $\mathrm{H}_{2}$ is further reused in other consumer plants whenever its purity is high enough (either directly or by means of the LPH or other headers), and if eventually the valve gets saturated, it is downgraded to fuel gas FG to be burnt in furnaces and boilers as a secondary alternative. In the MP/LP system of all consumer plants, the first branch opening under pressure control always goes to the FG header, whereas the last one is sent to the flare header.

Additionally, all consumer plants have optional HP purges to the FG header, opening only after all the previous branches in the split-range pressure control are already saturated; nevertheless, HP purges to FG are included in Table 1.1 only when being the first choice, otherwise their use is extremely infrequent.

In producer plant $\mathrm{H} 4$, part of the $\mathrm{H}_{2}$ produced is internally recycled, thus enabling to control the header $\mathrm{H} 4 \mathrm{H}$ pressure with the valve opening in the line where production is sent to header. In transient states, where production is increased before the consumption increases in consumer plants, the excess is downgraded to the header $\mathrm{H} 3 \mathrm{H}$ linked to producer plant $\mathrm{H} 3$, with the opening of the corresponding discharge valve under pressure control. In producer plant $\mathrm{H} 3$, part of the $\mathrm{H}_{2}$ produced is internally recycled, thus enabling to control the header $\mathrm{H} 3 \mathrm{H}$ pressure with the valve opening in the line where production is sent to header. An additional branch of the split-range pressure control purges the excess to FG, although it is rarely used. Catalytic reforming plants usually purge their excess to the LPH under pressure control, being the valves in the first branch to the downstream hydrotreater plant usually fully open (saturated). Therefore, control of the $\mathrm{H}_{2}$ production in producer plants is not difficult and can be decoupled from the global network. 
However pressure control regarding the $\mathrm{LPH}$, which is the link among consumer and producer plants in the network, enabling to reuse surplus $\mathrm{H}_{2}$, and moreover providing the necessary buffer capacity for control against uncertainty regarding the whole network, is more difficult. With the purpose of disturbance rejection regarding $\mathrm{H}_{2}$ consumption in the hydrotreating plants operation, as well as accounting for interactions among controllers in the multivariable problem of pressure control in the $\mathrm{LPH}$, it is mandatory to maintain a certain minimum flow rate as purge from the LPH to the fuel gas FG header. This minimum purge is intended to guarantee excess of hydrogen in spite of disturbances and interactions, i.e., safe operation for compressors without the risk of an emergency shut-down due to low pressure, and prevention of coke build-up on the catalyst due to lack of $\mathrm{H}_{2}$, for the global network. A MPC is in operation, designed and commissioned by the Petronor ${ }^{\circledR}$ Advanced Control Dpt., accounting for the multivariable nature of the network pressure control system and the significant interactions among plants connected to it. The MPC structure, manipulated and controlled variables, will not be detailed.

Operational risks are mainly due to pressure constraints in compressors. All compressors for make-up $\mathrm{H}_{2}$ are of the reciprocating type; only some of the recycle ones are centrifugal, but not all. Reciprocating compressors are designed to operate according to fix compression ratios, to balance the force exerted by the pistons thus improving mechanical stress and service life, being inlet and outlet pressures tightly controlled. The security locking system can be triggered because of low gas pressure, with the result of a compressor shut down and even unexpected sudden plant stop. An excess of gas pressure causes discharge valves and relief valves to open to fuel gas headers and flares, with the subsequent inefficient operation.

\subsection{4- Network optimal management}

$\mathrm{H}_{2}$ purity ( $y, \% 1 \mathrm{~mol}$ fraction) of gas streams used as make-up for the different consumer plants can typically vary from 0.65 to $1 \% 1$ mol. Total gas $\left(F, \mathrm{Nm}^{3} / \mathrm{h}\right)$ is used as synonym of non-pure $\mathrm{H}_{2}$, being light ends or impurities going with $\mathrm{H}_{2}$ in gas streams mainly methane, ethane and in a smaller ratio propane and heavier gases. The total make-up stream $\mathrm{H}_{2}$ purity can vary depending on the ratio of flow rates coming from the different producer plants $(\mathrm{H} 3$, H4, P1, P2) or distribution headers (low-purity header LPH) which are combined, being the cheapest either high-pressure HP surplus from other consumer plants or byproduct streams from the catalytic reforming units. The higher the $\mathrm{H}_{2}$ purity, the lower the gas flow rate needed as make-up. However, the higher the $\mathrm{H}_{2}$ purity, in general the higher the production cost. Therefore, a trade-off arises regarding efficiency: it increases with high $\mathrm{H}_{2}$ purity make-up when cost is not considered, although when $\mathrm{H}_{2}$ production costs are considered, the relation can be non-monotonous.

Hydrogen efficiency as a material resource is considered separately of the hydrocarbon treatment processes; the assumption is made that the reactor operation regarding desulphurization, de-nitrification, de-aromatization or hydrocracking remains unchanged irrespective of the $\mathrm{H}_{2}$ global redistribution, which is reasonable enough provided a minimum $\mathrm{H}_{2}$ purity is assured in the outlet gas from the HP separator and the $\mathrm{H}_{2}$ is in significant excess, thus being a non-limiting reactant. 
The efficiency of $\mathrm{H}_{2}$ as raw material is more meaningful at the global network scope (Fig. 1.5), where all the producer and consumer plants, as well as the available distribution headers and connecting pipes are considered. Optimal management of the $\mathrm{H}_{2}$ network refers to determining the optimal production rates in the producer plants $\mathrm{H} 3$ and $\mathrm{H} 4$, and the combination of make-up flows from each header providing $\mathrm{H}_{2}$ to each consumer plant, while fulfilling the operational constraints: i) a minimum ratio $\mathrm{H}_{2}$ /hydrocarbon must be ensured at the reactor inlet for each consumer plant; ii) a certain minimum $\mathrm{H}_{2}$ purity at the HP separator gas outlet must be assured for catalyst maintenance reasons; iii) capacity range for compressors, producer plants and pipes; iv) operating range and capacity for membranes; v) minimum purge to fuel gas to assure controllability and disturbance rejection in the Low Purity Header LPH.

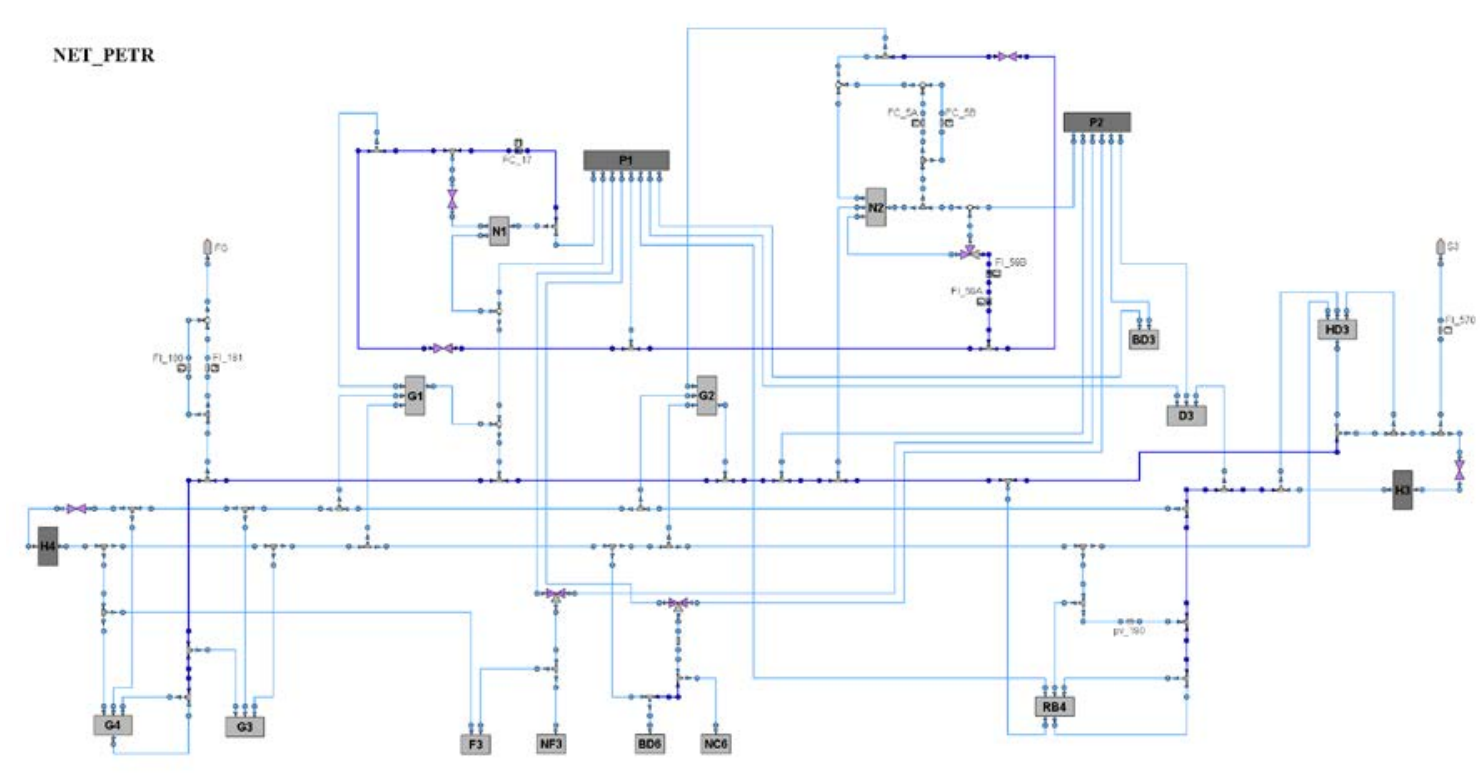

Fig. 1.5. Schematic of the Petronor ${ }^{\circledR}$ refinery $\mathrm{H}_{2}$ network.

\section{4- OUTCOME}

The outcome of the thesis comprises:

- a process simulation in the EcosimPro ${ }^{\circledR}$ environment for an industrial $\mathrm{H}_{2}$ network, with the same degrees of freedom and manipulated inputs as those physically available in the process. Besides, an implementation for the optimizations to solve the data reconciliation and optimal redistribution problems in EcosimPro ${ }^{\circledR}$ by using a Snopt ${ }^{\circledR}$ solver algorithm;

- a library developed in EcosimPro ${ }^{\circledR}$ for modeling $\mathrm{H}_{2}$ networks, which can be easily parameterized and configured. Furthermore, it includes additional features for the automatic generation of code to implement the optimization problems; it is described in Chapter 4; 
- a collaborative study for a revamp of the Petronor ${ }^{\circledR} \mathrm{H}_{2}$ network, leaded by the Repsol Technology Center (CTR) in Móstoles (Madrid) under a cooperation project with the Petronor ${ }^{\circledR}$ refinery from September 2013 to July 2014; final results were delivered by means of network simulations and optimizations built with the developed library in EcosimPro ${ }^{\circledR}$. As the study deals with optimization although from a design point of view, a brief summary is contained in Chapter 6 ;

- the problem of the $\mathrm{H}_{2}$ network optimal management -comprising the model, simulation and optimizations implementations- is being used as case study in the European project MORE "Real-time Monitoring and Optimization of Resource Efficiency in Integrated Processing Plants", from November 2013 until November 2016, intended for a real-time monitoring of the efficiency in the material and energy resources usage, and leaded by Prof. Sebastian Engell.

\section{5- PUBLICATIONS}

Publications in journals are under preparation:

"Data reconciliation and optimal management of hydrogen networks in a petrol refinery", 2012. Sarabia Ortiz, D., de Prada Moraga, C., Gómez Sayalero, E., Gutiérrez Rodríguez, G., Podar Cristea, S., Sola Sáez, J.M. et al. Control Engineering Practice, 20 (4), 343-354.

Publications in international conferences are listed below:

"Grey-box modelling of an industrial hydrodesulphurization process", 2008. Gómez, E., de Prada, C., Sarabia, D., Méndez, C.A., Cristea, S., Sola, J.M., Unzueta, E. $18^{\text {th }}$ European Symposium on Computer Aided Process Engineering - ESCAPE 18. Lyon, 2008. Editors Bertrand Braunschweig and Xavier Joulia. Elsevier B.V./Ltd. ISBN 978-0-444-53228-2.

"Optimal management of hydrogen supply and consumption networks of refinery operations", 2008. Méndez, C.A., Gómez, E., Sarabia, D., Cerdá, J., de Prada, C., Sola, J.M., Unzueta, E. $18^{\text {th }}$ European Symposium on Computer Aided Process Engineering - ESCAPE 18. Lyon. Editors Bertrand Braunschweig and Xavier Joulia. Elsevier B.V./Ltd. ISBN 978-0444-53228-2.

"Gestión óptima de redes de hidrógeno de refinerías", 2008. Gómez, E., de Prada, C., Sarabia, D., Méndez, C.A., Cristea, S., Sola, J.M., Unzueta, E. XXIX Jornadas de Automática, Tarragona.

"Grey-box modelling of an industrial hydrodesulphurization process", 2008. Gómez, E., de Prada, C., Sarabia, D., Méndez, C.A., Cristea, S., Sola, J.M., Unzueta, E. Reducit Workshop on Model Reduction for Industrial Control and Optimization Applications, 45 noviembre 2008, Frankfurt. 
"Data reconciliation and optimal management of hydrogen networks of a real refinery", 2009. Sarabia, D., Cristea, S., Gómez, E., Gutiérrez, G., Méndez, C.A., Sola, J.M., de Prada, C.. Proceedings of Adchem International Symposium on Advanced Control of Chemical Processes. Koc, Turkey.

"Simplified modelling and validation of an industrial diesel hydrodesulfurization plant", 2010. Gómez, E., Sarabia, D., Cristea, S., Gutiérrez, G., Méndez, C.A., Sola, J.M., Unzueta, E., González, R., de Prada, C. DYCOPS 9th International Symposium on Dynamics and Control of Process Systems. Lovaina, Bélgica, 5- 7 julio 2010.

"Self-optimizing control for hydrogen optimization in a diesel hydrodesulfurization plant", 2012. Sayalero, E.G., Skogestad, S., de Prada, C., Sola, J.M., González, R. PSE 11th International Symposium on Process Systems Engineering. Singapore, Singapore. July 15-19 2012. (Oral presentation).

"Optimal operation of a petrol refinery hydrogen network", 2014. E. Gómez, G. Gutiérrez, D. Sarabia, C. de Prada, S. Mármol, J.M. Sola, R. González. IFORS 20th Conference of the International Federation of Operational Research Societies. Barcelona, Spain, July 1318, 2014. (Oral presentation).

"Resource efficiency indicators applied to refinery hydrogen networks", 2015. E. Gómez, G. Gutiérrez, D. Sarabia, S. Mármol, J.M. Sola, C. Pascual, R. González, C. de Prada. ECCE 10th European Congress of Chemical Engineering. Nice, France, September 27thOctober 1st, 2015.

Award

Empresarios Agrupados E. A. Internacional 2008 award to the best work regarding models and/or libraries developed in the EcosimPro ${ }^{\circledR}$ modeling and simulation environment:

"Gestión óptima de redes de hidrógeno de refinerías", 2008. Gómez, E., de Prada, C., Sarabia, D., Méndez, C.A., Cristea, S., Sola, J.M., Unzueta, E. XXIX Jornadas de Automática, Tarragona, Spain, september 3-5, 2008. www.ecosimpro.com.

\section{6- OUTLINE}

The thesis is organized according to the following scheme.

After this introductory Chapter 1 , in Chapter 2 a simplified model for an industrial diesel hydrodesulfurizer (HDS) plant is developed and validated against real data. In Chapter 3 the self-optimizing control technique is applied to ascertain an efficient automatic control strategy to guarantee optimal performance regarding $\mathrm{H}_{2}$ consumption for the same HDS plant aforementioned, isolated from the global $\mathrm{H}_{2}$ network. In Chapter 4 , models for the different units are proposed with the aim of $\mathrm{H}_{2}$ optimal management, and assumptions justified. Besides, implementation issues in EcosimPro ${ }^{\circledR}$ are tackled. In Chapter 5 , the $\mathrm{H}_{2}$ network model 
is calibrated for different periods of operation against real data, by solving a data reconciliation problem with optimization techniques. In Chapter 6, the optimal management problem of the $\mathrm{H}_{2}$ network is formulated and solutions analysed. Finally, conclusions are summarized in Chapter 7. 


\section{Simplified Modelling ANd VALIDATION OF AN INDUSTRIAL Diesel HydRodesulfurizer Plant}

\section{1- INTRODUCTION}

A dynamic simplified model of an industrial diesel hydrodesulfurization plant has been developed and validated against plant data. Its purpose is the prediction of the rate of hydrogen consumption, as well as some other critical variables, as a function of the hydrocarbon feedstock, in order to gain insight into process operation as well as evaluate its potential integration in a decision support system aimed at the operation optimization in real time of the refinery hydrogen $\mathrm{H}_{2}$ network. A model which combines first physical-chemical principles with black box elements is proposed. Model structure and calibration procedure are described, and validation results are presented.

One of the most important types of $\mathrm{H}_{2}$ consumer plants are hydrodesulfurization units, whose purpose is to remove sulphur and other contaminants from a hydrocarbon stream. Due to the huge size of the problem and the scale it operates, detailed dynamic models of the units are not adequate. So, a simplified model for the dynamic prediction of the hydrogen consumption rate in a diesel hydrodesulfurization reactor is developed with the purpose of computing predictions of the $\mathrm{H}_{2}$ consumption rate as a function of the hydrocarbon load, to be integrated in a DSS for the $\mathrm{H}_{2}$ redistribution at the network level.

Furthermore, the severity of the operation (mainly reactor temperature) is manipulated by the operator so as to achieve the desired level of desulfurization, being sulfur concentration in the hydrocarbon outlet stream the target of the process. This severity also influences the $\mathrm{H}_{2}$ consumption rate, as well as contributes to catalyst deactivation, so it could be very useful to be able to operate the reactor at a severity as low as possible, as it would benefit $\mathrm{H}_{2}$ consumption requirements and also catalyst active life. 


\section{2- STATE OF THE ART}

Mechanistic mathematical models have mostly been reported to simulate the performance of pilot-plant reactors (Chowdhury et al., 2002), (Froment et al., 1994), (Korsten and Hoffmann, 1996), (Tsamatsoulis and Papayannakos, 1998), being static models. Some authors make an effort to develop a rigorous mathematical model accounting for all the major reactions and simulating the performance of both pilot and industrial scale reactors (Bhaskar et al., 2004), (Bellos, Papayannakos et al., 2005), but only a few are dynamic models (Mederos, Ancheyta et al., 2006).

Regardless of being the reactor the core of the process, a plantwide model must be considered because the way the reactor must be conducted is also the result of many other decisions and constraints on different elements of the plant. Nevertheless, and to the best of our knowledge, a plant-wide simplified model of a diesel hydrodesulfurization process, with the corresponding degrees of freedom and measured variables availability, have not been reported in the literature. Likewise, published industrial tests are limited to short time periods, not considering long periods of time such as months.

In order to obtain a balanced equilibrium between numerical simplicity and accuracy, a greybox model of the diesel desulfurization plant has been proposed. The grey-box model combines first principles with "pseudo" kinetic parameters that are estimated by means of a neural network. The model can then be used for prediction purposes as it captures the inherent behaviour of the system. Previous contributions to the grey-box modelling area (Georgieva, Meireles, Feyo d'Azevedo, 2003), (Laurent, Boyer, Gatina, 2000), (Chen, Bernard, Bastin, Angelov, 2000) have shown the feasibility of the proposed approach.

The Chapter is organized as follows: After the introduction, section 2 describes briefly the hydrodesulfurization plant and section 3 provides the basic elements of the model. Section 4 deals with its general structure, simulation and calibration, while validation results are presented in section 5 . 


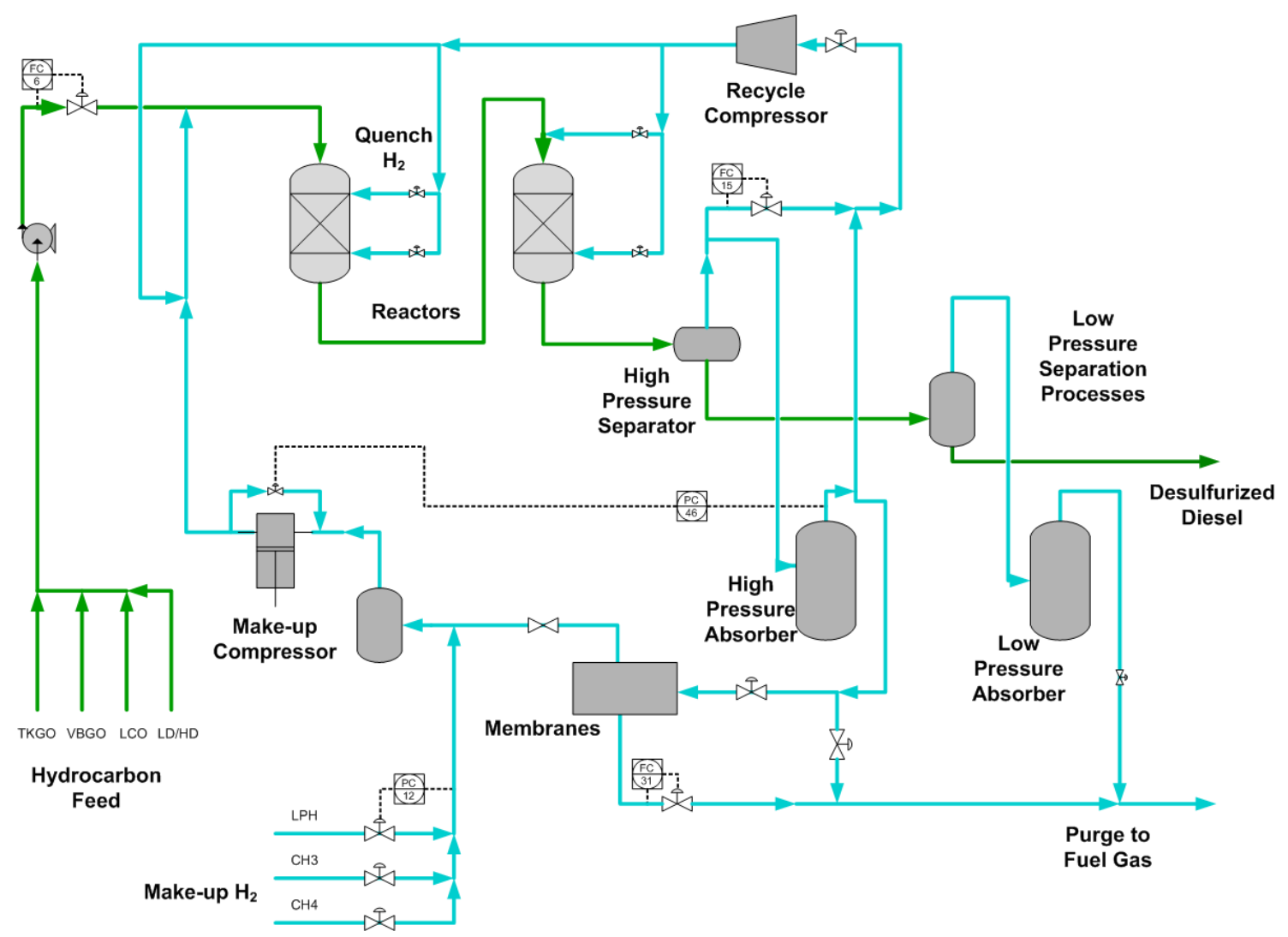

Figure 2.1. Simplified process flow diagram for a diesel hydrodesulfurizer plant with membranes.

\section{3- PROCESS DESCRIPTION}

The same process flow diagram of a HDS plant is shown in both Fig. 2.1 and Fig. 2.2. The desulfurization reactions take place in fixed bed catalytic reactors $(R 1, R 2)$ where a minimum ratio $\mathrm{H}_{2}$ /hydrocarbon must be ensured. The reactors are fed with a blend of fuels of different qualities $\left(F_{1}, F_{2}, F_{3}, F_{4}\right)$. Before entering the reactor, the feed is mixed with the recycled $\mathrm{H}_{2}$ stream and with a make-up $\mathrm{H}_{2}$ stream from the network. After being separated in a high pressure separation drum (D2), non-reacted $\mathrm{H}_{2}$ is partially recycled $\left(F_{144}\right)$, partially purified and recycled through membranes $Z 1\left(F_{30}\right)$, and partially burnt as fuel gas $\left(F_{31}, F_{46}\right)$. Reacted sulphur turns into $\mathrm{H}_{2} \mathrm{~S}$, which is removed by absorption on an amine solution (T3, T4), in absorbers operating at high and low pressure. Downstream of the high pressure separation drum D2, several distillation columns at lower pressure enable the complete separation of light gases from the desulfurized diesel stream. The stream of light gases from the low pressure separation processes is directed to fuel gas $\left(F_{32}\right)$, since its hydrogen purity is not high enough for making profitable the recovery. 


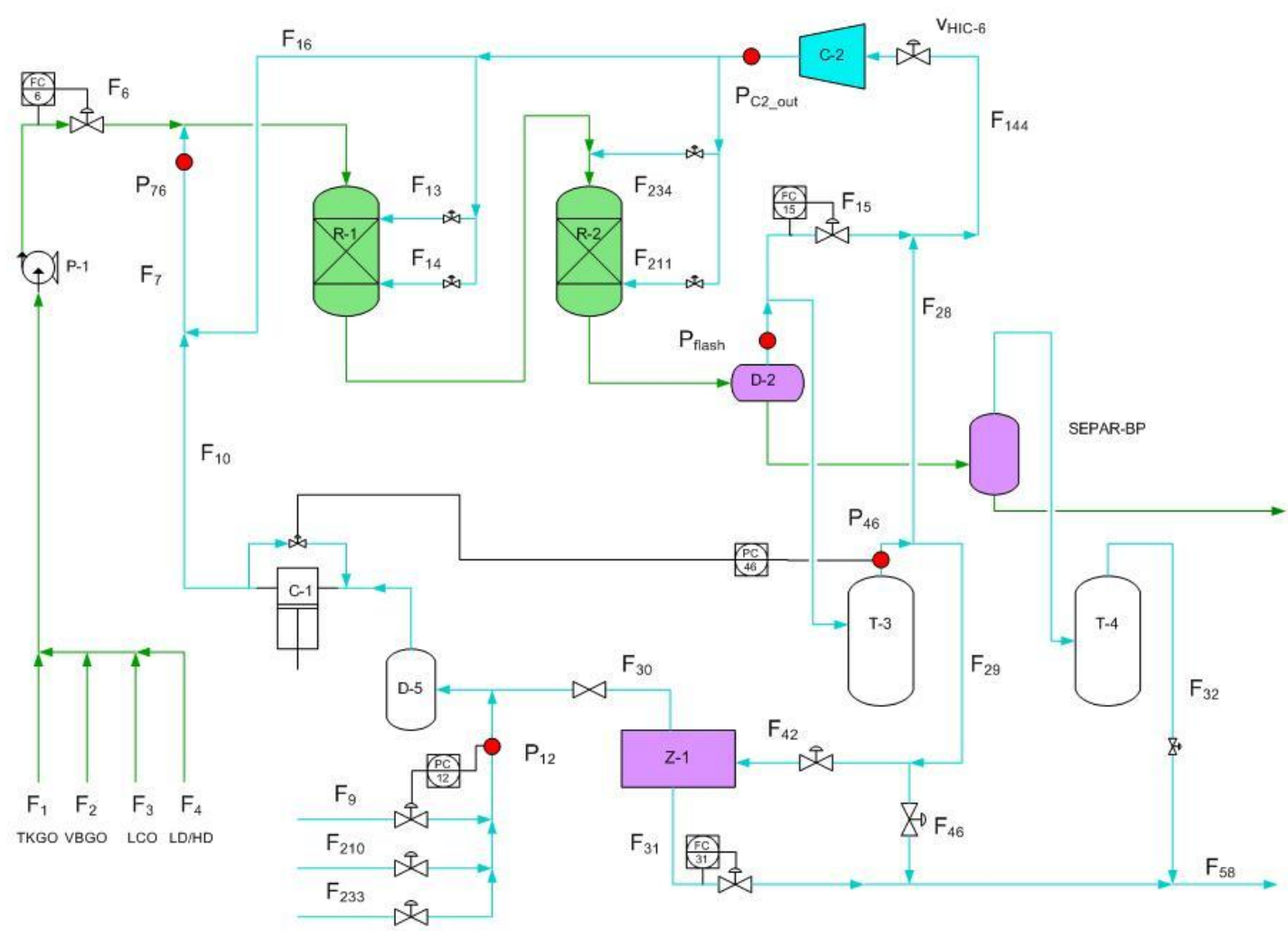

Figure 2.2. Simplified process flow diagram for a diesel hydrodesulfurizer plant with membranes.

\section{4- MODEL}

According to its purpose, only those units relevant for $\mathrm{H}_{2}$ consumption are considered in the model. These are the reactors, the high-pressure separator D2, the membranes, and, lumped into a single element, all of the low-pressure separation operations downstream of D2. All these units, as well as the material and momentum balances relating the flow between them, are described below. As temperature is controlled at the reactor inlet, between beds of the catalyst with quenching $\mathrm{H}_{2}$, and also before the high pressure separator, energy balances have not been considered because they do not influence $\mathrm{H}_{2}$ consumption and separation remarkably. Considering energy balances can provide an additional idea of the extent of the different reactions that are taking place, but introduces more complexity due to more unknown parameters (reactions and specific heats), so imposing the temperature as a manipulated boundary is close enough to reality.

\subsection{1- Pseudocomponents}

The thousands of components actually present in the hydrocarbon feed have been reduced to four named: hydrogen $\mathrm{H}_{2}$, light ends LIG (mainly methane $\mathrm{CH}_{4}$ and ethane $\mathrm{C}_{2} \mathrm{H}_{6}$, representing the rest of light components different from $\mathrm{H}_{2}$ in gas streams), sulphur $\mathrm{S}$, and the remaining 
hydrocarbons $\mathrm{HC}$, with the criterion that these are the key components from the point of view of $\mathrm{H}_{2}$ consumption and lumping the complex and changing mixture of hydrocarbons into a single one.

\subsection{2- Material balances}

Material balances for all plant nodes concerning gas streams are considered: total flow rate balance (eq. 1), hydrogen flow rate balance (eq. 2), and total mass flow rate balance due to the different molecular weight of the light gases that go with hydrogen depending on the particular stream (eq. 3). All the flowmeters provide flow measurements at Normal conditions, so the ideal gas equation of state can be assumed thus implying the equivalence between the canonical molar flow rate balances and the volumetric flow rate balances formulated.

$$
\begin{aligned}
& \sum_{i}^{I N} F_{i}=\sum_{j}^{\text {OUT }} F_{j} \quad \forall \text { node } \\
& \sum_{i}^{I N}\left(F_{i} \cdot y_{i}{ }^{H 2}\right)=y_{j}{ }^{H 2} \cdot \sum_{j}^{\text {OUT }} F_{j} \quad \forall \text { node } \\
& \sum_{i}^{I N} w_{i} \cdot\left(F_{i} / 22.415\right)=w_{j} \cdot \sum_{j}^{\text {OUT }}\left(F_{j} / 22.415\right) \quad \forall \text { node } \\
& \begin{array}{l}
1=y_{i}{ }^{H 2}+y_{i}{ }^{L I G} \quad \forall \text { stream } i \\
w_{i}=a_{i}+b_{i} \cdot y_{i}{ }^{H 2} \\
\forall i \quad \text { after separation }
\end{array}
\end{aligned}
$$

where $F_{i}$ refers to the volumetric flow rate of stream $i$ at Normal conditions $\left(\mathrm{Nm}^{3} / \mathrm{h}\right), y_{i}{ }^{\mathrm{H}}$ to the hydrogen purity of stream $i(\% 1 \mathrm{~mol})$, and $w_{i}$ to the molecular weight of stream $i(\mathrm{~kg} / \mathrm{kmol})$. Linear empirical correlations as (eq. 5) relating $\mathrm{H}_{2}$ purity and gas stream molecular weight are used for certain streams after undergoing a separation process, so as to take into account the different composition of impurities mainly due to the pressure of the separation process: the lower the pressure the higher the light ends molecular weight.

\subsection{3- Momentum balances}

Because of its critical role, momentum balance equations must be used to describe the equilibrium of flow and pressures achieved in the closed loop formed between the joint of the liquid and gas feeds upstream the reactor inlet, the reactor, downstream the reactor up to the high-pressure separator, and the line of the high pressure recycle stream driven by the centrifugal compressor C-2. In the remaining of the plant, the model does not go into this level of detail. Besides, there are manual valve openings without signal in the SCADA system. As the rest of the input boundaries concerning flows are linked to flow controllers, infeasibilities will be avoided in predictions by guaranteeing certain experimental relationships between the 
flows. For example, the inlet and outlet flows to the membranes unit must be within a certain range, obtained from historical experimental data.

The centrifugal compressor C-2 is described by means of the polytropic compression model (eq. 6), (eq. 7) and the manufacturer characteristic curve (eq. 8).

$$
\begin{aligned}
& H_{P}=Z_{a v} \cdot R \cdot T_{S} \cdot\left[\left(\frac{P_{D}}{P_{S}}\right)^{\frac{n-1}{n}}-1\right] /\left(w \cdot\left(\frac{n-1}{n}\right)\right) \\
& n=\left(\ln \left(P_{S} / P_{D}\right)\right) /\left(\ln \left(\left(P_{S} / P_{D}\right) /\left(T_{S} / T_{D}\right)\right)\right) \\
& H_{P}=a+b \cdot F_{S}+c \cdot F_{S}{ }^{2}+d \cdot F_{S}{ }^{3}
\end{aligned}
$$

where $S, D$ refers to suction and discharge of the compressor; $H_{P}$ is the polytropic head $(\mathrm{m}), P$ the pressure ( $\mathrm{Pa}), T$ the temperature $(\mathrm{K}), R$ the gas constant, $Z$ the generalized compressibility factor, and $F_{S}$ the volume flow rate at suction conditions. The polytropic coefficient $n$ can be obtained from measured data (eq. 7) and be supposed constant as the polytropic head is not quite sensitive to it, or else be linearly correlated with $\mathrm{H}_{2}$ purity.

Pressure drop across the reactor catalytic beds is described by the Ergun equation (eq. 10) for one phase and by the Larkins equation (eq. 11) for the two-phase flow, with Chisholm correlation. Pressure drop in lines is obtained by the approximate (eq. 9).

$$
\begin{aligned}
& \Delta P_{(G / L)}=f_{T} \cdot\left(L_{e} / D\right) \cdot\left(u_{S}{ }^{2} /(2 \cdot g)\right) \\
& \frac{\Delta P_{(G / L)}}{z_{R}}=\frac{1-\varepsilon}{\varepsilon^{3}} \cdot\left[a+\frac{b \cdot(1-\varepsilon) \cdot \mu}{d_{P} \cdot \rho \cdot u_{S}}\right] \cdot \rho \cdot \frac{u_{S}{ }^{2}}{d_{P}} \\
& \log \left(\Delta P_{2 f} /\left(\Delta P_{G}+\Delta P_{L}\right)\right)=0.416 /\left(\left(\log \left(\Delta P_{L} / \Delta P_{G}\right)^{1 / 2}\right)^{2}+0.666\right)
\end{aligned}
$$

where $\Delta P_{L}$ and $\Delta P_{G}$ refers to pressure drop due to one phase flow of liquid or gas, and $\Delta P_{2 f}$ to pressure drop due to two phase flow. $L_{e}(\mathrm{~m})$ is the pipe equivalent length, $D(\mathrm{~m})$ the diameter, $u_{S}(\mathrm{~m} / \mathrm{s})$ the fluid superficial velocity, $f_{T}$ the Fanning friction factor, and $g$ the gravity constant. The fixed bed reactor is characterized by the void fraction $\varepsilon()$, the length $z_{R}(\mathrm{~m})$, and the equivalent particle diameter $d_{p}(\mathrm{~m})$. The fluid density and viscosity are $\rho\left(\mathrm{kg} / \mathrm{m}^{3}\right)$ and $\mu\left(\mathrm{kg} \mathrm{m}^{-1} \mathrm{~s}^{-}\right.$ $\left.{ }^{1}\right)$.

\subsection{4- Reactor}

It is first considered the model describing the mixing of $\mathrm{H}_{2}$ and hydrocarbon feed in a two phase equilibrium. The gas-liquid equilibrium is described according to Riazi (2007). A transport delay is considered between the hydrocarbon feed and gas mixing and the reactor inlet.

$$
F_{6}+F_{G i n}=L_{R i n}+V_{R i n}
$$




$$
\begin{aligned}
& F_{6} x_{6}{ }^{j}+F_{G i n} y_{G i n}{ }^{j}=L_{R i n} X_{R i n}{ }^{j}+V_{R i n} y_{R i n}{ }^{j}, j=H_{2}, \text { LIG } \\
& F_{6} \cdot x_{6}{ }^{j}+F_{\text {Gin }} \cdot y_{\text {Gin }}{ }^{j}=L_{\text {Rin }} \cdot x_{\text {Rin }}{ }^{j} \quad j=S
\end{aligned}
$$

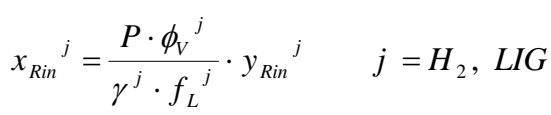

where $\operatorname{Rin}$ refers to reactor inlet, $F_{6}$ and $F_{\text {Gin }}$ are the mixed hydrocarbon and gas flows $(\mathrm{kmol} / \mathrm{h})$, $L$ and $V(\mathrm{kmol} / \mathrm{h})$ the liquid and gas flows entering the reactor in equilibrium, and $x^{j}$ and $y^{j}(\% 1$ $\mathrm{mol}$ ) the corresponding compositions of component $j$ in the liquid and gas phases.

Regarding the reactor, a full mechanistic model is too complex for the purpose intended of optimization in real time, together with the lack of available on-line measurements. The complexity is due to: i) the high number of reactions, where $\mathrm{H}_{2}$ is not only consumed in hydrodesulfurization reactions, but also in other hydrotreating reactions as hydrodenitrogenation and hydrodearomatization, and additionally in other side reactions as hydrocracking, hydrogenation of olefins, etc.; ii) the wide range of compounds in the hydrocarbon feed to the reactor; iii) the complex hydrodynamic in a multiphase reactor with trickle-bed flow; iv) the calculation of accurate thermodynamic and transport properties for such complex mixtures; v) the different stages which take place in series to the reaction: gasliquid and liquid-solid interphase mass and heat transfer; vi) and the fact that usually the rate equations available do not account for the competitive adsorption of other sulphur compounds, metals or hydrocarbons. Moreover, consideration of these phenomena will increase significantly the number of unknown parameters that should be estimated.

So a grey-box modelling approach, combining the basic global balances with black-box elements to avoid detailed and complex descriptions of intrinsic elements of the process has been adopted. In particular, reactions considered are the removal of sulphur (eq. 16), the consumption of $\mathrm{H}_{2}$ due to desulfurization and the consumption of $\mathrm{H}_{2}$ in the rest of side reactions (eq. 17), and the generation of light gases (eq. 18). The hydrodesulfurization reaction is modelled as first order with respect to sulphur, and all of the three reactions of zero order with respect to $\mathrm{H}_{2}$ assuming it is present in sufficient excess in the liquid phase:

$$
\begin{aligned}
& v_{L} \cdot \frac{d\left(c_{\text {Rout }}{ }^{s}\right)}{d t}=L_{\text {Rin }} \cdot x_{\text {Rin }}{ }^{s}-L_{\text {Rout }} \cdot x_{\text {Rout }} s-k_{S}{ }^{r} c_{\text {Rout }}{ }^{S} W_{\text {cat }} \\
& V_{L} \cdot \frac{d\left(C_{\text {Rout }}{ }^{H 2}\right)}{d t}=L_{\text {Rin }} \cdot x_{\text {Rin }}{ }^{H 2}-L_{\text {Rout }} \cdot x_{\text {Rout }}{ }^{H 2} \\
& -\alpha_{H 2-S} \cdot k_{S}{ }^{r} \cdot C_{\text {Rout }}{ }^{s} \cdot W_{\text {cat }}-k_{H 2}{ }^{r} \cdot W_{\text {cat }} \\
& +k_{H 2}{ }^{T M} \cdot\left(y_{\text {Rout }}{ }^{H 2}-y_{\text {Rout }}{ }^{H 2}{ }^{\text {eq }}\right) \\
& v_{L} \cdot \frac{d\left(c_{\text {Rout }}^{\text {LIG }}\right)}{d t}=L_{\text {Rin }} \cdot x_{\text {Rin }}^{\text {LIG }}-L_{\text {Rout }} \cdot x_{\text {Rout }}^{\text {LIG }} \\
& +k_{\text {lig }}{ }^{r} \cdot W_{\text {cat }}-k_{\text {CH4 }}{ }^{T M} \cdot\left(y_{\text {Rout }}{ }^{\text {LIG eq }}-y_{\text {Rout }}{ }^{\text {LIG }}\right)
\end{aligned}
$$

where Rin and Rout refers to reactor inlet and outlet, $v_{L}\left(\mathrm{~m}^{3}\right)$ is the liquid holdup in the reactor, $L(\mathrm{kmol} / \mathrm{h})$ is the flow of the liquid phase where the reactions take place, $c^{j}\left(\mathrm{kmol} / \mathrm{m}^{3}\right)$ is the 
concentration of component $j$ in the liquid phase, $k_{j}{ }^{T M}$ the mass transfer coefficient between gas and liquid for component $j, W_{\text {cat }}(\mathrm{kg})$ the active mass of catalyst -which will decrease in time-, and $\alpha_{\text {H2-s }}$ the stoichiometric coefficient relating $\mathrm{H}_{2}$ consumption in sulphur removal reactions, for which a constant value of 1.5 is supposed. A constant molecular weight $w^{H C}$ has been supposed for hydrocarbon. The mass transfer coefficients $k_{j}{ }^{T M}$ are assumed high enough so that phases can be considered in equilibrium.

No global mass accumulation is considered. The three kinetic parameters $k_{\mathrm{H} 2}{ }^{r}, k_{S}{ }^{r}, k_{\text {lig }}{ }^{r}$ are obtained from a neural network (NN) as a function of the main variables affecting the process: reactor temperature, $\mathrm{H}_{2}$ partial pressure, and type of hydrocarbon fed (diesel from straight run $F_{4}$, light cyclic oil from FCC unit $F_{3}$, diesel from visbreaker unit $F_{2}$, tank diesel $F_{1}$ ), being the proportions of each flow with respect to the total flow the regressors for the NN. It is known from experience that it is the type of hydrocarbon fed the factor that influences the most the $\mathrm{H}_{2}$ consumption in the reactor, much more than the quality of the crude oil being processed. Other equations in the reactor refer to the phase equilibria and mass transfer between the gas and the liquid phase (eq. 19, 20).

$$
\begin{aligned}
& V_{\text {Rin }} \cdot y_{\text {Rin }}{ }^{j}-V_{\text {Rout }} \cdot y_{\text {Rout }}{ }^{j}= \\
& k_{j}{ }^{T M} \cdot\left(y_{\text {Rout }}{ }^{j}-y_{\text {Rout }}{ }^{j \text { eq }}\right), \quad j=H_{2}, \text { LIG } \\
& x_{\text {Rout }}{ }^{j}=\frac{P \cdot{\phi_{V}}^{j}}{\gamma^{j} \cdot{f_{L}}^{j}} \cdot y_{\text {Rout }}{ }^{j \text { eq }} \quad j=H_{2}, \text { LIG }
\end{aligned}
$$

\subsection{5- High-pressure separator}

It is modelled according to the corresponding mass balances to the gas (eq. 21,22 ) and liquid phases (eq. 25, 26), phase equilibrium relationships (eq. 23) and transfer between phases (eq. 24).

$$
\begin{aligned}
& \frac{d}{d t}\left(\frac{P_{F} \cdot V_{V F}}{R \cdot T_{F}}\right)=\frac{V_{V F}}{R \cdot T_{F}} \cdot \frac{d}{d t}\left(P_{F}\right)=V_{\text {Rout }}+V_{V}-V_{F} \\
& \frac{V_{V F}}{R \cdot T_{F}} \cdot \frac{d}{d t}\left(P_{F} \cdot y_{F}^{j}\right)=V_{\text {Rout }} \cdot y_{\text {Rout }}^{j} \\
& +V_{V} \cdot y_{F}^{j e q}-V_{F} \cdot y_{F}^{j} \quad j=H_{2} \\
& x_{F}^{j}=\frac{P_{F} \cdot \phi_{V}^{j}}{\gamma^{j} \cdot f_{L}^{j}} \cdot y_{F}^{j e q} \quad j=H_{2}, \text { LIG } \\
& V_{V} \cdot{y_{V}}^{j}={k_{M T}}^{j} \cdot\left({y_{F}}^{j e q}-y_{F}^{j}\right) \quad j=H_{2}, L I G \\
& v_{L F} \cdot \rho_{L} \cdot \frac{d}{d t}\left(x_{F}^{j} / w_{L F}\right)=\frac{\rho_{L} \cdot F_{\text {Rout }}}{w_{\text {Rout }}} \cdot x_{\text {Rout }}{ }^{j} \\
& -\frac{\rho_{V} \cdot F_{V}}{w_{V}} \cdot y_{F}{ }^{j e q}-\frac{\rho_{L} \cdot F_{L F}}{w_{L F}} \cdot x_{F}^{j}, j=H_{2}, L I G, S
\end{aligned}
$$




$$
F_{L F}=F_{\text {Rout }}-F_{V}, \quad v_{L F}=\text { cte }
$$

where $F$ refers to the high pressure separator, $v_{V F}$ and $v_{L F}\left(\mathrm{~m}^{3}\right)$ are the volumes of the gas and liquid phases, $V_{V}(\mathrm{kmol} / \mathrm{h})$ is the flow of gas that undergoes phase change due to forcing function, $V_{F}(\mathrm{kmol} / \mathrm{h})$ the flow of gas that constitutes the drum outlet stream, $k_{M T}{ }^{j}(\mathrm{kmol} / \mathrm{h})$ the mass transfer coefficients, $F\left(\mathrm{~m}^{3} / \mathrm{h}\right)$ volumetric flows, and $w_{i}(\mathrm{~kg} / \mathrm{kmol})$ molecular weights. All the liquid densities $\rho_{L}\left(\mathrm{~kg} / \mathrm{m}^{3}\right)$ are supposed equal; the level of the liquid phase is controlled (26) and $F_{V}\left(\mathrm{~m}^{3} / \mathrm{h}\right)$ is the volume of the liquid phase corresponding to $V_{V}(\mathrm{kmol} / \mathrm{h})$.

\subsection{6- Membranes}

Disregarding mass accumulation, the membranes model can be considered just like another node in the network, with analogous material balances as the ones corresponding to (eq. 1), (eq. 2), (eq. 3), and the relation (eq. 4), and an extra prediction equation (eq. 27) obtained by a linear regression of experimental data. So, $\mathrm{H}_{2}$ purity of the permeate stream is obtained as a function of the $\mathrm{H}_{2}$ purity in the inlet stream and the ratio of the inlet flow purged to fuel gas.

$$
\hat{y}_{\text {PERMT }}{ }^{H 2}=f\left(\left(F_{\text {PURG }} / F_{I N}\right), \hat{y}_{I N}{ }^{H 2}\right)
$$

\subsection{7- Low pressure separations}

As a result of the low pressure separation processes, the liquid stream from the high pressure separator splits off into a liquid stream containing all of the $S$ and $H C$, and a gas stream containing all the $\mathrm{H}_{2}$ and LIG (light gases). The model also includes a transport delay and two first order lags in series, not shown here. Material balance model comprises (eq. 28-32).

$$
\begin{aligned}
& L_{L F}=F_{S H C}+\left(F_{L P} / 22.415\right) \\
& \left(F_{L P} / 22.415\right) \cdot y_{L P}{ }^{H 2}=L_{L F} \cdot x_{F}{ }^{H 2} \\
& \left(F_{L P} / 22.415\right)=L_{L F} \cdot x_{F}{ }^{H 2}+L_{L F} \cdot x_{F}{ }^{L I G} \\
& x_{S H C}{ }^{j}=0 \quad j=H_{2}, L I G \\
& F_{S H C} \cdot x_{S H C}{ }^{s}=L_{L F} \cdot x_{F}{ }^{s}
\end{aligned}
$$

where $F_{L P}\left(\mathrm{Nm}^{3} / \mathrm{h}\right)$ refers to the gas stream composed by all the $\mathrm{H}_{2}$ and light gases coming from the low pressure separation processes and going to fuel gas; that is, $F_{32}\left(\mathrm{Nm}^{3} / \mathrm{h}\right)$ in Fig. 2.2; and $F_{S H C}(\mathrm{kmol} / \mathrm{h})$ refers to the outlet liquid stream.

\section{5- MODEL CALIBRATION}


The DAE model comprises 13 differential equations and a total of 492 equations, including 2 nonlinear algebraic loops. Some of the state variables are pressures or correspond to a flow controller with negligible dynamic effects, just with the purpose of numerical decoupling. The model was simulated in the EcosimPro environment, which uses the DASSL algorithm for integration. The acceleration factor of the model execution speed with respect to real time is about 15000, with an Intel Core(TM)2 CPU @ 1.86GHz processor, 1.98GB RAM. A sequential approach has been followed for parameter estimation.

First, assuming that the dynamic in the establishment of flows and pressures is negligible compared to the dynamic of compositions, those parameters related to momentum balances have been estimated independently of the rest of the model, since enough measurements of flows and pressures are available. In particular, equivalent lengths of the pipes upstream and downstream the reactor; equivalent length of the recycle pipe; and particle diameter and void fraction of the catalyst bed. The correlation for $\mathrm{H}_{2}$ purity in the permeate stream in membranes was also estimated at this stage.

Secondly, the static inverse problem was solved fixing as inputs the following measured output variables of the system: $\mathrm{H}_{2}$ purity of the recycle stream from the high pressure separator, sulphur concentration of the desulfurized hydrocarbon stream and flow rate of the $\mathrm{H}_{2}$ makeup. In this way, the three kinetic coefficients of the reaction rates can be obtained: $k_{S}{ }^{r}, k_{H 2}{ }^{r}, k_{\text {lig }}{ }^{r}$. Three independent neural networks are then trained in Matlab with the toolbox NNSYSID 2.0 (Norgaard, 2000). The best results are achieved with recursive NOE models of order one, with architectures of 3 hyperbolic tangent neurons in the hidden layer and 1 linear neuron in the output layer. This enables a first approximate value of the NN parameters.

Finally, the whole set of unknown parameters is estimated, including those directly related to dynamic effects: volumes involved in transport delays, liquid phase volume in the reactor and in the high pressure separation drum. This is solved in EcosimPro using a SQP NAG ${ }^{\circ}$ routine as solver. Large series of historical data, without experimental design, expanding over a month have been used. In this way, sensitivities with respect to dynamic parameters are low due to large time scales compared to sensitivities with respect to parameters which influence the gains of the process. The general structure of the model and the calibration procedure is shown in Fig.2.3.

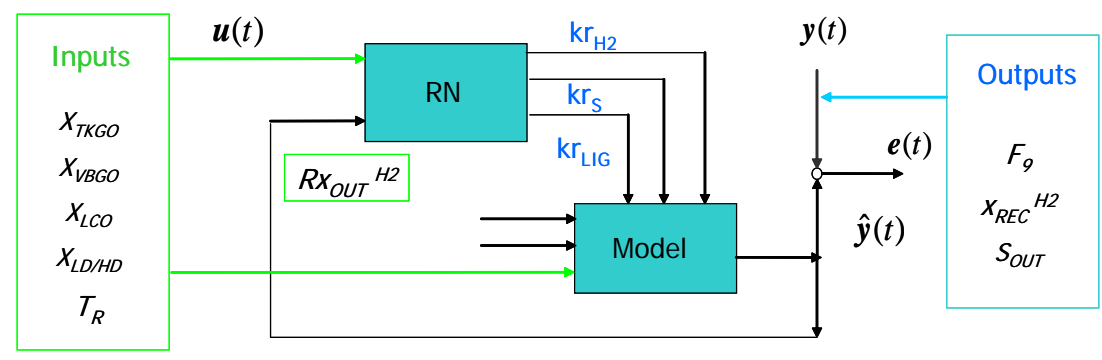

Fig. 2.3. Structure for dynamic parameter estimation. 


\section{6- MODEL VALIDATION}

The following figures correspond to model validation for one month of operation, with blue representing model output and green experimental data. For confidentiality reasons, all the variables appear in $\%$ of a certain scale.

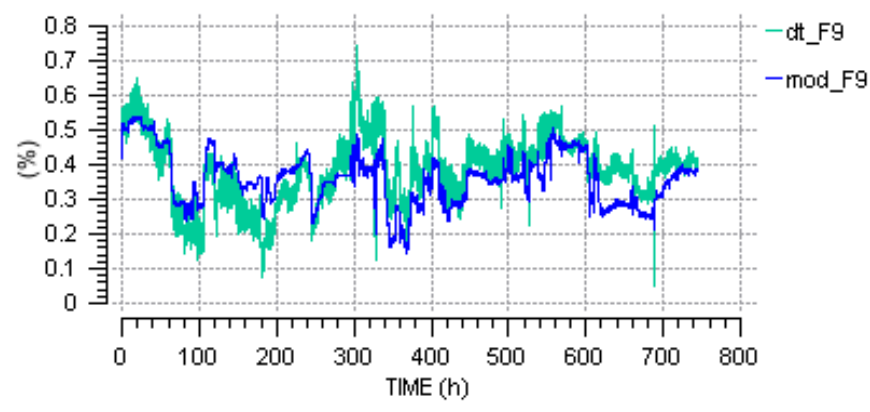

Fig. 2.4. Make-up $\mathrm{H}_{2}$ from low purity pipe.

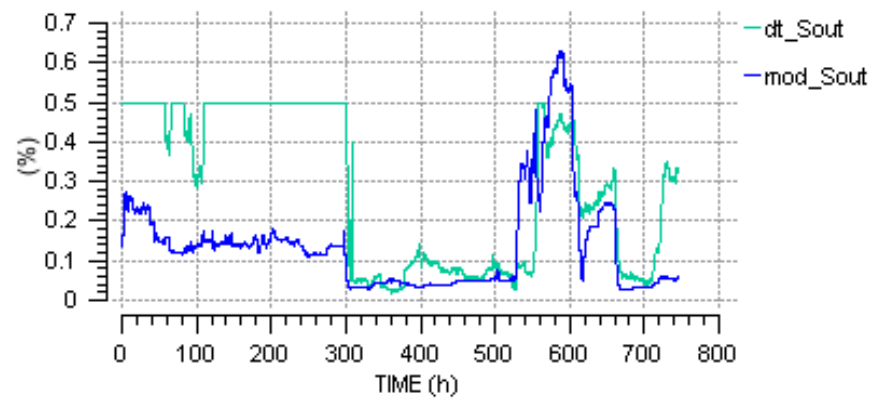

Fig. 2.5. Sulfur concentration in diesel desulfurized stream (analyser not in operation for 0-300 h).

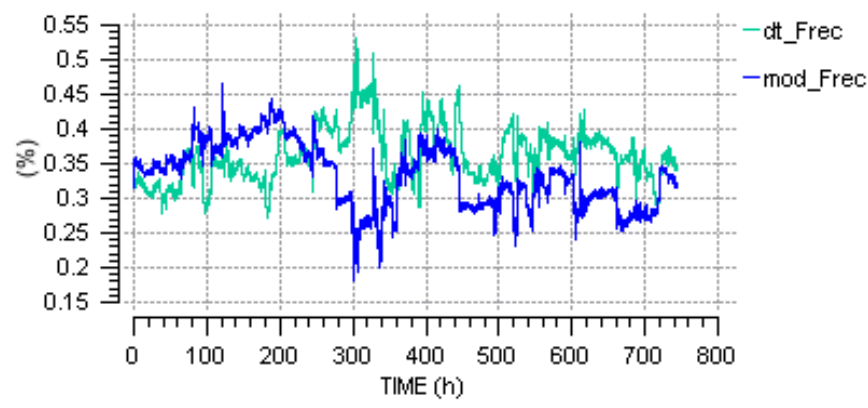

Fig. 2.6. Flow of the recycle stream from the high pressure separator. 


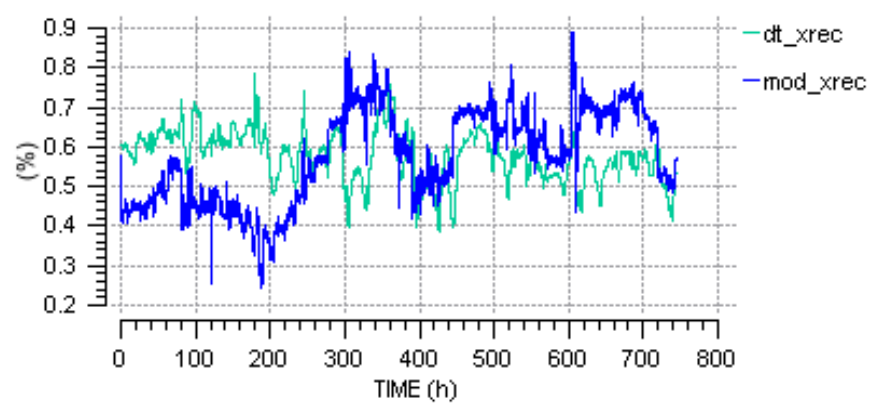

Fig. 2.7. $\mathrm{H}_{2}$ purity of the recycle stream from the high pressure separator.

The most interesting output of the model, the flow rate of $\mathrm{H}_{2}$ make-up, is estimated with less than $10 \%$ error, while good results are also obtained for the sulphur concentration in the desulfurized diesel stream. It should be noticed that they have been obtained without a good knowledge of the feedstock sulphur composition and make-up $\mathrm{H}_{2}$ purity, since no on-line measurement is available, so that perfect results can never be expected. Similar results have been obtained for other periods of operation.

\section{7- CONCLUSIONS}

A dynamic simplified model of a diesel HDS plant has been validated against real data. In spite of the uncertainty in some periods, results are good enough so as to validate the use of this kind of models in a decision support tool.

Despite the simplicity of the model, predictions for $\mathrm{H}_{2}$ consumption rate are found to agree well with the experimental data in the range of operating conditions studied, although predictions for sulphur concentration are less accurate. Further work should be done so as to refine some of the hypothesis now considered. In particular, the $\mathrm{H}_{2}$ purity of the low purity make-up will not be constant and the material balances model for the whole $\mathrm{H}_{2}$ network should be included in order to better estimate its value. Moreover, an observer-based estimator could be incorporated to improve the knowledge of another important unknown inlet, the sulphur concentration of the hydrocarbon feed. The assumption of zero-order kinetics for $\mathrm{H}_{2}$ could be as well reviewed.

Although further improvements should be made before the model could be embedded in a decision support tool, two potential uses of the model are now possible. The first one is the prediction of the $\mathrm{H}_{2}$ consumption rate as a function of the hydrocarbon load, so as to take decisions on the rates of production in the $\mathrm{H}_{2}$ producer plants and on the $\mathrm{H}_{2}$ redistribution at the network level. The second one is the internal plant operation optimization, in particular of the degree of severity in the operation of the reactor (temperature) so as to fulfil the specification of desulfurization being as close as possible to the limit in the most efficient way. This is particularly important, because high temperatures cause an increase in carbon deposition in the catalyst, with the subsequent and undesirable decrease in catalyst activity. 


\section{CHAPTER 3}

\section{Self-Optimizing Control for $\mathrm{H}_{2}$ Optimization in AN INDUStRIal Diesel HydROdesUlfurizer PlANT}

\section{1- INTRODUCTION}

The aim of this Chapter is to apply the self-optimizing control technique (Skogestad, 2000) in order to ascertain the plantwide control structure for a hydrodesulfurizer HDS plant of a petroleum refinery with regards to hydrogen consumption optimization. The resulting control structure for the HDS plant is simple, robust to uncertainty, easy to implement (feedback control) and assures the global optimum in most cases, although an upper RTO layer will be needed to guarantee the operation in the adequate region, with no frequent updates. Only in one uncommon scenario a trade-off arises regarding the unconstrained degrees of freedom, and self-optimizing control variables must be looked for. Potential application to the global refinery $\mathrm{H}_{2}$ network is also discussed.

Determining a good plantwide control structure, in this case for an $\mathrm{H}_{2}$ consumer diesel hydrodesulfurizer HDS plant, is an issue of great practical importance to achieve optimal operation. Skogestad's procedure looks for a control strategy which can be implemented in practice in a robust and simple manner. In the first place, active constraints are controlled, and then, for the remaining unconstrained economic degrees of freedom, self-optimizing variables are looked for. Self-optimizing control (Skogestad, 2000) is when close-to-optimal economic operation is obtained with a constant set-point policy. In this way it is avoided, or at least reduced, the need for an upper optimization RTO layer, being feedback control still the easiest way to implement a control strategy in industrial practice (Skogestad, 2004). Self-optimizing control design has been applied successfully to many processes, e.g. Araujo, Govatsmark and Skogestad (2007); Lid and Skogestad (2008). 


\section{2- GENERAL PROBLEM DESCRIPTION}

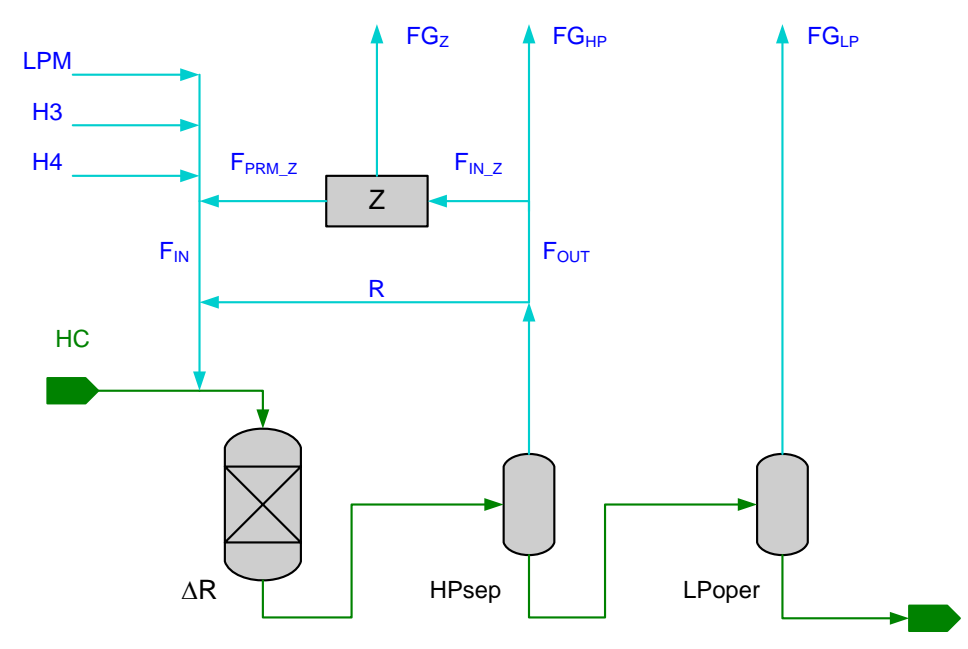

Figure 3.1. General $\mathrm{H}_{2}$ consumer plant structure, with membranes unit for $\mathrm{H}_{2}$ recovery.

The process flow diagram for the hydrodesulfurizer HDS plant is shown in Fig. 3.1. The HDS plant simplified model is the one developed and explained in Chapter 2, being $F_{i}\left(\mathrm{Nm}^{3} / \mathrm{h}\right)$ flow rates. The objective is to minimize operating costs, which will be the cost of the make-up $\mathrm{H}_{2}$ in a first approach. It is worthy to mention certain remarks:

- In descending order of profitability, two main sources are available for $\mathrm{H}_{2}: \mathrm{LPH} \mathrm{H}_{2}$, cheap low-purity $\mathrm{H}_{2}$, excess from other consumer plants or by-product of catalytic-reforming units; and $\mathrm{HPH} \mathrm{H}_{2}$, expensive, high-purity $\mathrm{H}_{2}$ produced in steam-reforming furnaces producer plants from methane. Regarding nomenclature, LPH stands for the low pressure header, source of low-purity $\mathrm{H}_{2}$, whereas HPH stands for the two high pressure headers corresponding to $\mathrm{H} 4$ and $\mathrm{H} 3$ producer plants, sources of high-purity $\mathrm{H}_{2}$. Whenever feasible according to the constraints, operation with the highest $\mathrm{LPH} \mathrm{H}_{2}$ flow rate will be preferred, because of its low cost as compared to $\mathrm{HPH} \mathrm{H}_{2}$.

- A trade-off can arise with regard to the $\mathrm{H}_{2}$ make-up from the different high-purity producer plants, because a higher flow rate will be needed of the cheapest one, assuming the cheapest corresponds to the one with lower purity among the $\mathrm{HPH}$ sources of $\mathrm{H}_{2}$.

- If a purge stream is needed to maintain the high pressure HP system $\mathrm{H}_{2}$ purity above the lower limit (main operating constraint), removing light ends entering with the make-up and generated in the reactor, then it is more profitable to purge from the membranes $F_{F G_{-} Z}$ than directly from the high pressure system $F_{F G_{-} H P \text {. }}$ According to the material balance for a closed control volume comprising the HP 
system, since continuity exists in the gas phase across the reactor and the high pressure HP separator, an increase of $1 \mathrm{Nm}^{3} / \mathrm{h}$ in the purge either from $F_{F G_{-} Z}$ or $F_{F G_{-} H P}$ leads to an increase of $1 \mathrm{Nm}^{3} / \mathrm{h}$ in the make-up flow rate. However, $\mathrm{H}_{2}$ purity of $F_{F G_{-} Z}$ is always lower than $\mathrm{H}_{2}$ purity of $F_{F G_{-} H P}$, thus improving $\mathrm{H}_{2}$ use and reducing $\mathrm{H}_{2}$ purged to fuel gas.

\section{3- SELF-OPTIMIZING CONTROL STRUCTURE}

The systematic procedure for plantwide control (Skogestad, 2004) will be applied to determine the self-optimizing control structure, following the steps in the top-down economic steadystate analysis.

\subsection{1- Degrees of freedom analysis}

A total of 5 degrees of freedom are available for economic optimization based on the process model. The following are natural manipulated variables $(u)$ in operation:

- $\mathrm{H}_{2}$ make-up flow rates from producer plants $F_{H 3}, F_{H 4}$;

- membranes inlet flow rate $F_{I N \_} z ;$

- membranes purge ratioed to the inlet $F_{F G_{Z} Z} / F_{I N_{Z} z} z$

- direct purge to fuel gas from the high pressure system $F_{F G_{-} H P \text {. }}$

Other process variables are not truly degrees of freedom regarding $\mathrm{H}_{2}$ optimization; that is the case for: a) pressure in separators, controlled at a fixed value according to compression ratios and security valves design; b) temperature in separators, regulated at minimum possible value by means of air-cooled heat exchangers, thus enabling the best relative separation of $\mathrm{H}_{2}$ from light ends; c) quench streams between beds in the catalytic reactors, whose purpose is to maintain proper temperature gradients and to avoid high temperatures in reactor beds. They are determined under temperature control, thus not being manipulated variables, and the current model does not take into account their influence over reactor operation; d) reactor inlet temperature, which will always be active constraint at the minimum value enabling to achieve the sulphur specification in diesel product. The minimum possible temperature is desired for various reasons: the extension of side reactions (like hydrocracking) is reduced, and as a consequence catalyst active life is extended and consumption of $\mathrm{H}_{2}$ is reduced; besides, furnace operating cost is minimized, where fuel to furnace is the manipulated variable.

\subsection{2- Definition of optimal operation: cost and constraints}

Target is minimization of the operating cost. A first analysis will be carried out where only material costs of $\mathrm{H}_{2}$ are considered. Usually $\mathrm{H}_{2}$ cost is an order of magnitude higher than compression energy cost, so the simplified approach is justified as a first step. Membranes operating costs can be disregarded as only low pressure steam is needed. 
Hydrogen from the LPH has zero cost as compared to $\mathrm{H}_{2}$ from steam-reforming furnaces producer plants, as it is a byproduct of many plants operation. Nevertheless, when $\mathrm{H}_{2}$ from producer plants $\left(\mathrm{HPH} \mathrm{H}_{2}\right)$ is not needed to satisfy the operational constraints, the target would be to use as less LPH $\mathrm{H}_{2}$ as possible, so that excesses in the LPH are available for other consumer plants, and in this way improving the use. That is the reason why a term taking into account the cost of $\mathrm{LPH} \mathrm{H} \mathrm{H}_{2}$ is considered in the proposed objective function. This LPH fictitious price should be fixed in order to achieve good numerical properties for the optimization problem; low enough so as to not compete with $\mathrm{HPH} \mathrm{H}_{2}$, but not zero either. A price $p_{L P H}$ equal to zero would lead to a necessary change in the objective function when no make-up from the $\mathrm{HPH}$ is required, minimizing in this case the LPH flow rate.

Although fuel gas value of the purge streams from the high and low pressure separation operations could be considered in the cost objective function $\left(p_{f u e l}\right)$, this term will compete with minimization of the LPH make-up $\mathrm{H}_{2}$ flowrate. Moreover, $\mathrm{LPH} \mathrm{H}_{2}$ price is already a fictitious one, as it is difficult to quantify the value of a common intermediate utility which can replace $\mathrm{HPH} \mathrm{H}_{2}$ in many consumer plants leading to a reduced $\mathrm{HPH} \mathrm{H}_{2}$ production. As a consequence, only make-up $\mathrm{H}_{2}$ cost will be taken into account:

$$
\min _{u} J=p_{H 4} \cdot F_{H 4}+p_{H 3} \cdot F_{H 3}+p_{L P H} \cdot F_{L P H}
$$

Constraints to fulfil are the following:

- HP system (or recycle) $\mathrm{H}_{2}$ purity between minimum to prevent deposition of coke over catalyst particles ensuring enough $\mathrm{H}_{2}$ excess, and maximum to avoid surge of centrifugal compressor: $\min <y_{R E C}<\max$;

- membranes operating range and membranes capacity: $\min <F_{F G_{-} z} / F_{I N_{-} Z}<\max$, $\min <F_{I N \_}<\max ;$

- $\quad$ producer plants capacity and LPH availability: $\min <F_{\mathrm{H}_{3}}<\max , \min <F_{H_{4}}<\max , 0<$ $F_{L P H}<\max$

- compressors [reciprocating/centrifugal] capacity: $\min <F_{C}<\max$;

- the constraint $\mathrm{H}_{2}$ /hydrocarbon minimum ratio at reactor inlet, again to assure excess of $\mathrm{H}_{2}$ in the reactor: $F_{\mathrm{H} 2} / F_{\mathrm{HC}}>$ min. Since the $\mathrm{H}_{2}$ recycle flow rate is much greater than the $\mathrm{H}_{2}$ make-up flow rate, typically 3 to 4 times, it is attained mainly with the recycle compressor. The recycle compressor's capacity is controlled at the minimum feasible to minimize the compression energy cost, being thus decoupled from the $\mathrm{H}_{2}$ material optimization problem.

\subsection{3- Identification of important disturbances}

Regarding the plant state, disturbances in hydrocarbon feedstock and process operation influence the gas separated in the low pressure operations $F_{F G_{-} L P,} Y_{F G_{-} L P}{ }^{H 2}$, as well as the 
hydrogen consumption $\Delta R_{H 2}$ and light ends generation $\Delta R_{L I G}$ in the reactor. Regarding different future scenarios, there are several disturbances that deserve being considered, namely:

- the $\mathrm{H}_{2}$ purity of certain make-up streams $\mathrm{y}_{L P H}{ }^{{ }^{H} 2}, \mathrm{y}_{H 3}{ }^{{ }^{2} 2}$;

- the make-up flow rate $F_{L P H}$ availability from the $L P H$, as it depends on other $\mathrm{H}_{2}$ consumer plants;

- the price of the HPH $\mathrm{H}_{2}$ from the steam-reforming furnaces producer plants. Prices per total flow rate $\left(€ / \mathrm{Nm}^{3}\right)$ are considered.

\subsection{4- Regions of active constraints (modes of operation)}

To identify the different regions of active constraints, optimization with respect to the available degrees of freedom is performed for the different operating points or scenarios (19) selected corresponding to historical process conditions, for 6 case studies corresponding to different disturbances in utilities (see below); in total 114 case studies. Upper and lower bounds on decision variables and constraints are fixed according to process knowledge. The model is implemented in EcosimPro modelling and simulation environment and optimizations are performed with a NAG ${ }^{\circ}$ SQP solver linked to EcosimPro .

1. Base case;

2. A high decrease of $15 \%$ in the $\mathrm{LPH} \mathrm{H}_{2}$ purity;

3. A small decrease of $5 \%$ in the $\mathrm{LPH} \mathrm{H} \mathrm{H}_{2}$ purity;

4. A decrease in the availability of $\mathrm{LPH} \mathrm{H}_{2}$, so that it becomes active constraint;

5. An increase of $5 \%$ in the purity of one of the sources of $\mathrm{HPH}$, that whose $\mathrm{H}_{2}$ purity is smaller;

6. A decrease in the $\mathrm{H}_{2}$ price for one of the sources of $\mathrm{HPH}$, that whose $\mathrm{H}_{2}$ purity is smaller.

In the optimal solutions found, all the active constraints are first determined, because they should be controlled to achieve optimal operation (Maarleveld and Rijnsdorp, 1970) due to their high economic penalty. Consequently, the number of steady-state degrees of freedom minus the number of active constraints constitutes the remaining number of unconstrained degrees of freedom. For these unconstrained degrees of freedom, an equal number of selfoptimizing controlled variables can be proposed, constituting a candidate set of controlled variables. Self-optimizing controlled variables need to be found for each region of active constraints.

In increasing order of $\mathrm{H}_{2}$ requirements, the regions of active constraints found are the following: 
- A. Recycle $\mathrm{H}_{2}$ purity $\left(y_{R E C}{ }^{H 2}=\min \right)$ controlled with $F_{I N_{Z} z}$. Manipulated variables active: $F_{F G_{-}} / F_{I N_{-} Z}=\min , F_{F G_{-} H P}=0, F_{H 3}=0, F_{H 4}=0$.

\begin{tabular}{lccccc}
\hline Manipulated variables & $F_{I N_{-} Z}$ & $\begin{array}{c}F_{F G_{-} Z} / F_{I N_{-} z} \\
=\min \end{array}$ & $\begin{array}{c}F_{F G_{-} H P} \\
=0\end{array}$ & $\begin{array}{c}F_{H 3} \\
=0\end{array}$ & $\begin{array}{c}F_{H 4} \\
=0\end{array}$ \\
\hline $\begin{array}{l}\text { Controlled variables } \\
\text { (constraints) }\end{array}$ & $\begin{array}{l}y_{R E C}{ }^{H 2} \\
=\min \end{array}$ & $r_{H_{-} H_{C}}$ & $F_{C 1}$ & $F_{L P H}$ & \\
\hline
\end{tabular}

Purge is needed to achieve the minimum recycle purity constraint, because of the low LPH make-up $\mathrm{H}_{2}$ purity. Recycle $\mathrm{H}_{2}$ purity is controlled with $F_{I N \_z}$. Higher inlet to membranes implies higher $\mathrm{H}_{2}$ make-up flow rate, so higher operation cost. Lower inlet to membranes implies recycle $\mathrm{H}_{2}$ purity lower than the specified minimum, so infeasible operation. This is the most common case in operation.

- B. Recycle $\mathrm{H}_{2}$ purity $\left(y_{R E C}{ }^{H 2}=\min \right)$ controlled with $F_{F G_{-} Z} / F_{I N_{-} Z}$. Manipulated variables active: $F_{I N_{-} Z}=\max , F_{F G_{-} H P}=0, F_{H 3}=0, F_{H 4}=0$.

\begin{tabular}{llllll}
\hline Manipulated variables & $\begin{array}{c}F_{I N_{-} Z} \\
=\max \end{array}$ & $F_{F G_{-} Z} / F_{I N_{-} Z}$ & $\begin{array}{c}F_{F G_{-} H P} \\
=0\end{array}$ & $\begin{array}{l}F_{H 3} \\
=0\end{array}$ & $\begin{array}{l}F_{H 4} \\
=0\end{array}$ \\
\hline $\begin{array}{l}\text { Controlled variables } \\
\text { (constraints) }\end{array}$ & $\begin{array}{l}y_{R E C}{ }^{H 2} \\
=\min \end{array}$ & $r_{H_{-} Z_{C}}$ & $F_{C 1}$ & $F_{L P H}$ & \\
\hline
\end{tabular}

When $\mathrm{H}_{2}$ requirements increase with respect to the case $A$, the $\mathrm{H}_{2}$ purge $\left(F_{F G_{-} Z} / F_{I N_{-} Z}\right)$ is not at the lower bound because the membranes capacity upper bound is reached and a higher purge is needed. Higher ratio $\left(F_{F G_{-} Z} / F_{I N_{-} z}\right)$ implies higher permeate purity according to the membranes model, but at the expense of a higher make-up $\mathrm{H}_{2}$ flow rate. Recycle $\mathrm{H}_{2}$ purity is controlled with the ratio $F_{F G_{-} Z} / F_{I N_{-} Z}$.

The amount of pure $\mathrm{H}_{2}$ that can be lost to fuel gas by increasing the ratio $F_{F G_{Z} Z} / F_{I N_{-} z}$ is $21 \%$ of the total pure $\mathrm{H}_{2}$ inlet to membranes when operating at maximum capacity and a certain $\mathrm{H}_{2}$ purity of $50 \%$, and it is profitable provided that the maximum membranes capacity is already binding constraint and LPH $\mathrm{H}_{2}$ availability is assured (see Table below, where all variables have been scaled according to a certain operating range). Permeate stream purity $y_{P R M_{-}}{ }^{\mathrm{H} 2}$ has 
been computed according to the membranes model (linear empirical correlation depending on $y_{I N_{-} Z}{ }^{H 2}$ and the ratio $\left.F_{F G_{-} Z} / F_{I N_{-} Z}\right)$.

$$
y_{P R M_{-} Z}{ }^{H 2}=a \cdot\left(F_{F G_{-} Z} / F_{I N_{-} Z}\right)+y_{I N_{-} Z}{ }^{H 2}+b
$$

\begin{tabular}{|c|c|c|c|c|}
\hline & & $\begin{array}{l}\text { Lower } \\
\text { bound }\end{array}$ & $\begin{array}{l}\text { Upper } \\
\text { bound }\end{array}$ & Diference \\
\hline$F_{F G_{-} Z} / F_{I N_{-} Z}$ & () & $0 \%$ & $100 \%$ & \\
\hline$F_{I N \_Z}$ & $\left(\mathrm{Nm}^{3} / \mathrm{h}\right)$ & $100 \%$ & $100 \%$ & \\
\hline$F_{F G_{Z} Z}$ & $\left(\mathrm{Nm}^{3} / \mathrm{h}\right)$ & $22 \%$ & $45 \%$ & \\
\hline$F_{P R M \_} z$ & $\left(\mathrm{Nm}^{3} / \mathrm{h}\right)$ & $78 \%$ & $55 \%$ & \\
\hline$y_{I N \_} Z^{H 2}$ & (\%1 mol) & $50 \%$ & $50 \%$ & \\
\hline$y_{F G_{-} Z}{ }^{H 2}$ & (\%1 mol) & $17 \%$ & $37 \%$ & \\
\hline$y_{P R M_{Z} Z}{ }^{H 2}$ & (\%1 mol) & $78 \%$ & $86 \%$ & \\
\hline$F_{F G_{-} Z} \cdot y_{F G_{-} Z}{ }^{H 2}$ & $\left(\mathrm{Nm}^{3} \mathrm{H}_{2} / \mathrm{h}\right)$ & $11 \%$ & $33 \%$ & $21 \%$ \\
\hline$F_{P R M} z \cdot y_{P R M} z^{H 2}$ & $\left(\mathrm{Nm}^{3} \mathrm{H}_{2} / \mathrm{h}\right)$ & $88 \%$ & $67 \%$ & $-21 \%$ \\
\hline
\end{tabular}

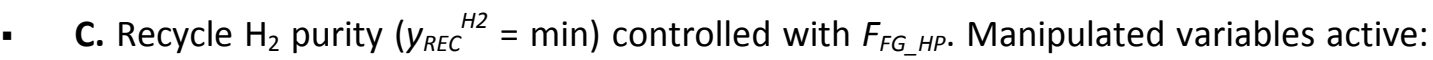
$F_{I N_{-} Z}=\max , F_{F G_{-} Z} / F_{I N_{-} Z}=\max , F_{H 3}=0, F_{H 4}=0$.

\begin{tabular}{lccccc}
\hline Manipulated variables & $\begin{array}{c}F_{I N_{-} Z} \\
=\max \end{array}$ & $\begin{array}{c}F_{F G_{-} Z} / F_{I N_{-} z} \\
=\max \end{array}$ & $F_{F G_{-} H P}$ & $\begin{array}{c}F_{H 3} \\
=0\end{array}$ & $\begin{array}{c}F_{H 4} \\
=0\end{array}$ \\
\hline Controlled variables & $y_{R E C}{ }^{H 2}$ & $r_{H 2} H C$ & $F_{C 1}$ & $F_{L P H}$ & \\
(constraints) & $=\min$ & & & & \\
\hline
\end{tabular}

When $\mathrm{H}_{2}$ requirements increase with respect to the previous case $B$, the minimum $Y_{R E C}{ }^{H 2}$ cannot be achieved only with the membranes since they are saturated. Provided that $\mathrm{LPH} \mathrm{H}_{2}$ availability is assured, the direct purge to fuel gas from the high pressure system $F_{F G_{-} H P}$ is increased to fulfil the constraint on $y_{\text {REC }}{ }^{\mathrm{H} 2}$.

- D. Trade-off between ratio purged in membranes and HPH $\mathrm{H}_{2}$ make-up $\left(F_{H 4}, F_{F_{-} Z} / F_{I N_{-} Z}\right)$. Recycle $\mathrm{H}_{2}$ purity controlled $\left(y_{R E C}{ }^{H 2}=\min \right)$. Manipulated variables active: $F_{I N_{-} z}=\max$, $F_{F G_{-} H P}=0, F_{H 3}=0$. Case rather infrequent. 


\begin{tabular}{llllll}
\hline Manipulated variables & $F_{I N_{-} Z}$ & $F_{F G_{-} Z} / F_{I N-} Z$ & $F_{F G_{-} H P}$ & $F_{H 3}$ & $F_{H 4}$ \\
& $=\max$ & & $=0$ & $=0$ \\
\hline Controlled variables & $y_{R E C}^{H 2}$ & $r_{H_{2} H_{C}}$ & $F_{C 1}$ & $F_{L P H}$ \\
(constraints) & $=\min$ & & & \\
\hline
\end{tabular}

When make-up $\mathrm{H}_{2}$ from a producer plant $\left(\mathrm{HPH} \mathrm{H}_{2}\right)$ is needed due to $\mathrm{LPH} \mathrm{H}_{2}$ shortage (small purity for example but not small $F_{L P H}$ availability); then purge by means of membranes will mean an increase both in $\mathrm{H}_{2}$ make-up flow rate and in $\mathrm{H}_{2}$ losses to fuel gas, and as a result an increase in cost if $\mathrm{HPH} \mathrm{H}_{2}$ is needed; a trade-off arises. The optimum is quite flat with respect to $F_{F G_{-} Z} / F_{I N_{-} Z}$, so a value can be fixed while using $F_{H 4}$ to control $y_{R E C}{ }^{H 2}$, being $F_{F G_{Z} Z} / F_{I N_{Z} Z}=0.33$ the most common by far (self-optimizing variable). According to the experiments carried out, the optimal ratio varies between $0.27-0.36$, so 600 $\mathrm{Nm}^{3} \mathrm{H}_{2} / \mathrm{h}$ is the difference for $F_{I N_{Z} Z}=\max$, and the maximum loss in $\mathrm{HPH} \mathrm{H}_{2}$ is $300 \mathrm{Nm}^{3} / \mathrm{h}$ provided 0.33 is fixed. Cases $B$ and $D$ can be easily distinguished; if an increase in $F_{L P H}$ (by allowing an increase in $F_{F G_{-}} / F_{I N_{-} Z}$ ) does not lead to an increase in $y_{R E C}{ }^{H 2}$, then HPH H$H_{2}$ will be needed. This occurs when $y_{L P H}{ }^{H 2}<y_{R E C}{ }^{H 2}$. Direct purge $F_{F G_{-} H P}$ will be needed if membranes get saturated, besides $\mathrm{HPH} \mathrm{H}_{2}$.

- E. When the maximum make-up compressor's capacity is binding constraint besides $y_{R E C}{ }^{H 2}\left(y_{R E C}{ }^{H 2}=\min , F_{C 1}=\max \right)$, controlled with $F_{F G_{-} H P}$ and $F_{H 4}$. Manipulated variables active: $F_{I N_{-} Z}=\max , F_{F G_{Z} Z} / F_{I N_{-} Z}=\max , F_{H 3}=0$.

\begin{tabular}{lccccc}
\hline Manipulated variables & $\begin{array}{c}F_{I N_{-} Z} \\
=\max \end{array}$ & $\begin{array}{c}F_{F G_{-} Z} / F_{I N_{-} Z} \\
=\max \end{array}$ & $F_{F G_{-} H P}$ & $F_{H 3}$ & $F_{H 4}$ \\
& $y_{R E C}{ }^{H 2}$ & $r_{H 2} H C$ & $F_{C 1}$ & $F_{L P H}$ \\
Controlled variables & $=\min$ & & $=\max$ & \\
(constraints) & & & \\
\hline
\end{tabular}

This situation is common in cases where $y_{L P H}{ }^{H 2}>y_{R E C}{ }^{H 2}$, but only slightly greater. As a high flow $F_{L P H}$ is needed, make-up compressor capacity gets saturated. As usual, membranes capacity is fully employed before $\mathrm{HPH} \mathrm{H}_{2}$ is used. It will be uncommon such a high availability of low purity $\mathrm{H}_{2}$.

The minimum required recycle $\mathrm{H}_{2}$ purity $\left(y_{R E C}{ }^{H 2}\right)$ cannot be achieved only with the membranes. $F_{F G_{-} H P}$ is increased until $F_{C 1}$ is active constraint (so that $F_{L P H}$ enters accordingly under pressure control), and $F_{H 4}$ is increased until $y_{R E C}{ }^{H 2}$ is active constraint (decreasing $F_{L P H}$ accordingly). In spite of the multivariable nature of the control problem, small interactions between loops are expected. 
- F. When the maximum LPH H$H_{2}$ availability is active constraint, besides $y_{R E C}{ }^{H 2}\left(y_{R E C}{ }^{H 2}=\right.$ $\left.\min , F_{L P H}=\max \right)$. Manipulated variables active: either $F_{F G_{Z} Z} / F_{I N_{-} Z}=\min , F_{F G_{-} H P}=0, F_{H 4}=0$ (control with $\left.F_{I N_{-} Z}, F_{H 3}\right) ; F_{F G_{-} Z} / F_{I N_{-} Z}=\min , F_{F G_{-} H P}=0, F_{H 3}=0$ (control with $\left.F_{I N_{-} Z}, F_{H 4}\right) ; F_{I N_{-} Z}=$ $\max , F_{F G_{Z} Z} / F_{I N_{-} Z}=\min , F_{F G_{-} H P}=0$ (control with $\left.F_{H 3}, F_{H 4}\right)$; or $F_{I N_{-} Z}=\max , F_{F G_{-} H P}=0, F_{H 3}=0$ (control with $F_{F G_{-} Z} / F_{I N_{-} Z}, F_{H 4}$ ).

\begin{tabular}{|c|c|c|c|c|c|}
\hline Manipulated variables & $\begin{array}{l}F_{I N \_Z} \\
=0\end{array}$ & $\begin{array}{l}F_{F G_{-} Z} / F_{I N_{-} Z} \\
=\min \end{array}$ & $\begin{array}{l}F_{F G_{-} H P} \\
=0\end{array}$ & $F_{H 3}$ & $F_{H 4}$ \\
\hline Manipulated variables & $F_{I N \_} z$ & $\begin{array}{c}F_{F G_{-} Z} / F_{I N_{-} Z} \\
=\min \end{array}$ & $\begin{array}{c}F_{F G_{-} H P} \\
=0\end{array}$ & $F_{H 3}$ & $\begin{array}{l}F_{H 4} \\
=0\end{array}$ \\
\hline Manipulated variables & $F_{I N \_Z}$ & $\begin{array}{c}F_{F G_{-} Z} / F_{I N_{-} Z} \\
=\min \end{array}$ & $\begin{array}{l}F_{F G_{-} H P} \\
=0\end{array}$ & $\begin{array}{l}F_{H 3} \\
=0\end{array}$ & $F_{H 4}$ \\
\hline Manipulated variables & $\begin{aligned} & F_{I N \_Z} \\
= & \max \end{aligned}$ & $F_{F G_{-} Z} / F_{I N_{-} Z}$ & $\begin{array}{c}F_{F G_{-} H P} \\
=0\end{array}$ & $F_{H 3}$ & $\begin{array}{l}F_{H 4} \\
=0\end{array}$ \\
\hline Manipulated variables & $\begin{aligned} & F_{I N \_Z} \\
= & \max \end{aligned}$ & $F_{F G_{-} Z} / F_{I N \_} Z$ & $\begin{array}{c}F_{F G_{-} H P} \\
=0\end{array}$ & $\begin{array}{l}F_{H 3} \\
=0\end{array}$ & $F_{H 4}$ \\
\hline $\begin{array}{l}\text { Controlled variables } \\
\text { (constraints) }\end{array}$ & $\begin{array}{l}Y_{R E C}^{H 2} \\
=\min \end{array}$ & $r_{H 2}{ }_{H C}$ & $F_{C 1}$ & $\begin{aligned} & F_{L P H} \\
= & \max \end{aligned}$ & \\
\hline
\end{tabular}

Both membranes and $\mathrm{HPH} \mathrm{H}_{2}$ make-up are needed. With increasing $\mathrm{H}_{2}$ demand, first $F_{I N_{Z} Z}$ is manipulated, then $F_{F G_{Z} Z} / F_{I N_{-} Z}$. Between the two $\mathrm{HPH} \mathrm{H} \mathrm{H}_{2}, F_{H 3}$ and $F_{H 4}$, which one is used depends mainly on their respective prices and purities (very constant), but also on plant state, although no trade-off arises. An upper RTO layer will be required to know whether to use $F_{H 3}$ or $F_{H 4}$, with very low frequent updates.

We note that in the first case no purge is needed, which means that all light gases either entering with make-up $\mathrm{H}_{2}$ or generated in the reactor come out dissolved in the high pressure separator liquid stream.

Furthermore, it is not clear in certain regions whether to use $\mathrm{HPH} \mathrm{H}_{2}$ from $\mathrm{H} 3$ or from $\mathrm{H} 4$ producer plants. It depends on their respective prices and purities, and also on $\Delta R_{H 2}, \Delta R_{L I G}, \mathrm{H}_{2}$ solved in the HP separator outlet liquid stream, and $y_{L P H}{ }^{H 2}$. As no one of these last four variables is measured, and must be obtained in a previous reconciliation stage, the decision making procedure can be difficult. An upper RTO layer would be adequate to update the binding 
manipulated variables, regarding the use of either $\mathrm{H} 3$ or $\mathrm{H} 4$ producer plants. Except for these no frequent updates, global optimality would be assured in most scenarios.

- G. When the minimum ratio $\mathrm{H}_{2} / \mathrm{HC}$ is active constraint; it is not a frequent occurrence.

\begin{tabular}{lccccc}
\hline Manipulated variables & $\begin{array}{c}F_{I N_{-} Z} \\
=\max \end{array}$ & $\begin{array}{c}F_{F G_{-} Z} / F_{I N_{-} Z} \\
=\min \end{array}$ & $F_{F G_{-} H P}$ & $\begin{array}{c}F_{H 3} \\
=0\end{array}$ & $\begin{array}{c}F_{H 4} \\
=0\end{array}$ \\
\hline Controlled variables & $y_{R E C}{ }^{H 2}$ & $r_{H 2} H C$ & $F_{C 1}$ & $F_{L P H}$ & \\
(constraints) & & $=\min$ & & & \\
\hline
\end{tabular}

As the ratio $\mathrm{H}_{2} / \mathrm{HC}$ is binding constraint, the maximum $\mathrm{H}_{2}$ flow at reactor inlet is desired, so purge in membranes is at the lower bound in order to maximize recycle from membranes. The remaining purge required to increase $\mathrm{H}_{2}$ make-

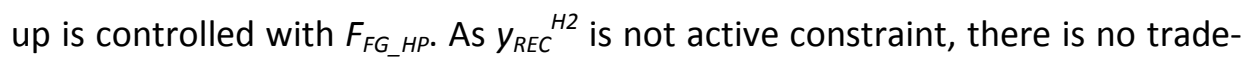
off.

\begin{tabular}{|c|c|c|c|c|c|}
\hline Manipulated variables & $F_{I N \_Z}$ & $\begin{array}{c}F_{F G_{-} Z} / F_{I N_{-} Z} \\
=\min \end{array}$ & $\begin{array}{l}F_{F G_{-} H P} \\
=0\end{array}$ & $F_{H 3}$ & $\begin{array}{l}F_{H 4} \\
=0\end{array}$ \\
\hline Manipulated variables & $\begin{aligned} & F_{I N \_} Z \\
= & \max \end{aligned}$ & $\begin{array}{l}F_{F G_{-} Z} / F_{I N_{-} Z} \\
=\min \end{array}$ & $F_{F G_{-} H P}$ & $F_{H 3}$ & $\begin{array}{l}F_{H 4} \\
=0\end{array}$ \\
\hline $\begin{array}{l}\text { Controlled variables } \\
\text { (constraints) }\end{array}$ & $y_{R E C}{ }^{H 2}$ & $\begin{array}{l}r_{H 2}{ }_{H C} \\
=\min \end{array}$ & $F_{C 1}$ & $\begin{aligned} & F_{L P H} \\
= & \max \end{aligned}$ & \\
\hline
\end{tabular}

Additionally, as $F_{L P H}$ is also active constraint, $F_{H 3}$ is increased afterwards being the cheapest one compared with $F_{H 4}$, because $y_{R E C}{ }^{H 2}$ is not an issue.

\subsection{5- Analysis of results}

The most important regions of active constraints have been identified and analysed, although it is clear that other sets not considered here could take place occasionally, depending on disturbances and economic conditions (prices). There are a great number of regions of active constraints despite the few manipulated and controlled variables. As a result, the implementation of the resulting control structure, with pairings between manipulated and controlled variables, will not be easy. Besides, implementation error is expected to be high due to transport delay. 
However, although the resulting control structure seems to be simple, the change in the set of active constraints needs to be considered and this could be difficult to implement. Perhaps the required logic to decide the pairings between manipulated and controlled variables could be programmed in an upper layer at the DCS level, connecting set-points, measurements and control signals so as to close loops accordingly along time. A split-range control structure could be proposed for each region of active constraints regarding certain manipulated variables: first control with membranes inlet $F_{I N}$, when saturated control with membranes purge $F_{F G_{-} Z}$, when saturated control with direct purge $F_{F G_{-} H P \text {. }}$

For every region, certain manipulated variables can be determined in a bound of the operating range, and the rest paired frequently with an active constraint. Recycle $\mathrm{H}_{2}$ purity $\mathrm{Y}_{R E C}{ }^{\mathrm{H}}$ is nearly always active constraint; although variables with good controllability should be chosen, $\mathrm{Y}_{\mathrm{REC}}{ }^{\mathrm{H}}$ is the natural controlled variable for economic optimization, to avoid a gap with respect to the minimum specification; its bad dynamic is due to a large transport delay involving the reactor, the high pressure separator and the recycle stream.

According to the aforementioned results, in only two regions, none of them being very common, a trade-off between two manipulated variables arises. Therefore, only in two regions there is a need to find a self-optimizing control variable to make use of the remaining unconstrained economic degrees of freedom. As a consequence, global optimal operation is ensured even in the presence of disturbances in most scenarios, all except for two regions with low probability.

\section{4- CONCLUSIONS}

Self-optimizing control is a robust technique not sensitive to uncertainty when compared with RTO (Skogestad, 2000). As i) disturbances: $\mathrm{H}_{2}$ consumption, light gases inlet and generation, and gases solubility in hydrocarbons, are difficult to estimate accurately, and ii) gas flow measurements need to be compensated with molecular weight in operation, where the stream molecular weight is quite sensitive to light gases composition due to the low value of $\mathrm{H}_{2}$ molecular weight $(2.016 \mathrm{~kg} / \mathrm{kmol})$, a self-optimizing control approach can be advantageous and preferred compared to RTO. Other major advantage is that the self-optimizing approach considered is reliable regardless of model mismatch: although model validation is not perfect quantitatively (errors up to $10 \%$ in the prediction of the $\mathrm{H}_{2}$ consumption rate), the analysis performed regarding regions of active constraints is correct.

The resulting control structure for the HDS plant is simple, easy to implement (feedback control with programmed logic) and assures the global optimum in most cases, although an upper RTO layer will be needed to guarantee the operation in the adequate region, with no frequent updates. Only in one scenario, rather uncommon, a trade-off arises regarding the unconstrained degrees of freedom, and self-optimizing control variables must be looked for to assure close to optimal operation avoiding more complex on-line optimization techniques. Main drawbacks are related to plant automation level, which needs to be high to implement this technology; in particular the membranes unit can't be operated manually. Transport delay 
due to reactor and dynamic effects due to separators and recycle are not negligible, therefore a MPC approach with an economic objective function could be justified, especially when the high number of regions of active constraints is taken into account, due to its potential to easily handle constraints.

A further step is being considered regarding the potential application of the self-optimizing control design to the on-line optimization of the global $\mathrm{H}_{2}$ network operation. At network scale, the optimal self-optimizing policy applied for every isolated consumer plant will lead in general to suboptimal network operation, because interactions may not be fully exploited. In general, a RTO approach should be applied at network scale. Nevertheless, although the structure at network scale is much more complex, the number of combinations allowed is usually small, and self-optimizing control structures could be looked for aiming at a simplified problem at the network scale, and thus reducing the loss between RTO executions. 


\section{CHAPTER 4}

\section{HYDROGEN NetWORK MODEL}

\section{1- INTRODUCTION AND STATE OF THE ART}

Model development for large scale applications is widespread, not only regarding statistical black-box models easier to generate and maintain, but also for first-principles models based on conservation balances and constitutive laws. Particularly in oil refineries, commercial modelbased applications exist for data reconciliation of hydrocarbon streams and steam networks, usually with simplified approaches. The use of first-principles models to support process operations has been applied in the chemical and petroleum industry for over 40 years. Typical on-line applications of these models range from real-time optimization, MPC, data reconciliation, soft sensors, process performance monitoring and others.

A review on the use of phenomenological first principles models in process operations can be seen in (Pantelides and Renfro, 2013). Key factors determining the modelling approach are complexity, solvability, maintainability and tractability issues. Also, model development cost can be a factor in considering the type of model used in these applications. Hence many simplified and empirical model-based online applications became preferred in some domains, even though the overall prediction quality of the first principles model may be superior. In general, a good balance between a reasonable accuracy regarding the process and acceptable computational efforts is looked for.

Faber, Arellano-Garcia and Wozny (2007) present an optimization framework for parameter estimation of large-scale systems. Esche, Müller, Kraus and Wozny (2014) present a systematic workflow for process systems engineers developing models for optimization purposes, since the main difficulties lie in the non-linearity, non-convexity and sheer size of the existing process models. The presented systematic leads to a successful implementation of process models applicable for optimization, which are both reduced in size, non-linearity, and nonconvexity.

This thesis fits within the interests of the "Process Control and Supervision" research group of the Dpt. of Systems Engineering and Automatic Control of the University of Valladolid, with significant experience in the areas of modelling and process simulation, process advanced control, process optimization, process supervision and industrial informatics. The group 
cooperates in developments and technological transfers with different companies (Empresarios Agrupados ${ }^{\circledR}$, Repsol ${ }^{\circledR}$, Petronor $^{\oplus}$, Acor $^{\circledR}$, Ebro $^{\circledR}$ ) and maintains an active collaboration with international institutions (Dortmund University, Carnegie Mellon University and others). As illustrative examples and references for this work, it is worthy to mention recent thesis in the research group: "SIMPD: An intelligent modelling tool for dynamic processes" (Felipe Acebes, César de Prada, 1996); "Modeling, simulation and non-linear predictive control of a distillation column of a petrochemical process" (Almudena Rueda, César de Prada, 2006); "Modeling library of the beet section of a sugar factory for an operator training simulator" (Alejandro Merino, Felipe Acebes, 2008); "Modeling library of the sugar house of a sugar factory for an operator training simulator" (Rogelio Mazaeda, César de Prada, 2010); "Modelling, simulation and advanced control using renewable energies and hybrid control of small-scale reverse osmosis desalination plants" (Luis Gómez Palacín, Fernando Tadeo, César de Prada, 2014); "Control and optimization of natural gas networks" (Mar Valbuena, Daniel Sarabia, César de Prada, in process); "NMPC for cooling strings of superconducting magnets using superfluid helium" (Rafal Noga, César de Prada, Enrique Blanco, 2015). In all of them, problems of industrial significance are addressed by means of modeling, optimization and advanced control techniques, with the general purpose of process operation improvement, resource (material and/or energy) saving, or process knowledge enhancement. In some of them commercially available modeling libraries in EcosimPro ${ }^{\circledR}$ were developed.

\section{2- HYDROGEN NETWORK MODEL}

The purpose of the model, intended for $\mathrm{H}_{2}$ operational optimization at the network scope, has determined the modelling approach. Only a simple material balance model is formulated, due to the following reasons:

- Momentum balances are not modelled in order to avoid complexity and a high level of detail and granularity, therefore decision variables are not valve openings but flow rates in themselves. Constraints regarding pipes capacity have been specified with historical data instead of being the result of the pressure-flow rate relationships, which is accurate enough according to the intended aim.

- Model complexity has been chosen according to the available on-line measurements; otherwise non-observable subsets of variables would be present, which will be difficult to relate with an underlying model due to feedstock complexity and process variability. That is the reason why energy balances have not been included in the model.

First, the general modelling assumptions will be stated, and next the model described by individual unit operations, only considering those units and phenomena related with $\mathrm{H}_{2}$ streams: gas streams pipes, reactors, separators, membranes, compressors, mixers and 
splitters, and valves. The material balance model for all unit operations is a simplified one, again with the target of making easier on-line model calibration previous to the operational optimization step.

The following general assumptions are considered:

- Dynamic effects are negligible, i.e. steady state conditions are satisfied over the averaged period. For important headers as those linked to producer plants, where pressure oscillates within a certain range in spite of being controlled and with greater pipe diameters and lengths, the mass accumulation can be around 1-2\% of the total gas driven, because of the different pressure in the header at the beginning and the end of the average time. This error is acceptable according to the standard deviation for flow rates, the magnitude is the same as that of instrument noise even in the worst case, therefore not influencing results to a significant extent.

Regarding local redundancy that occurs in nodes for gas streams, accumulation is limited and negligible for the common pipe dimensions, consequently it is not expected to influence the reconciled results for flow measurements.

In the high pressure HP system of consumer plants, dynamic effects are related with transport delays and the recycle stream from the HP separator gas outlet; the steady-state model is fed with average values for two-hour periods, which is higher than the transport delay thus encompassing dynamic effects to a certain extent.

- It is not distinguished whether the gas is either totally solved in the liquid or constituting a two-phase mixture; mass balances apply in both cases.

\subsection{1- Gas streams Model}

System model consists of material balances for gas streams, assumed to be constituted by two components: hydrogen $\mathrm{H}_{2}$, and the rest of light ends different from $\mathrm{H}_{2}$ in gas streams (mainly methane $\mathrm{CH}_{4}$, in a smaller ratio ethane $\mathrm{C}_{2} \mathrm{H}_{6}$, and others) lumped into another one named LIG. Although the individual components forming LIG will not be considered, the change in the LIG molecular weight $w^{L G}$ will be taken into account because: i) it is needed and important for online flow rate compensation as will be explained later, due to the orifice-plate flow meters available; ii) appreciable differences can be found in the $w^{L / G}$, mainly depending on the gas stream pressure and $\mathrm{H}_{2}$ purity; iii) in certain streams, especially in the HP system where it is more meaningful, the $w^{L / G}$ can be accurately estimated, with low process variability. As a consequence, three mass balances, one total and two individual ones, are required to model gas streams.

It is the mass balance what is truly satisfied, equivalent to the mole balance when no chemical reaction occurs. In the refinery, gas flow meters provide a raw measurement in volumetric

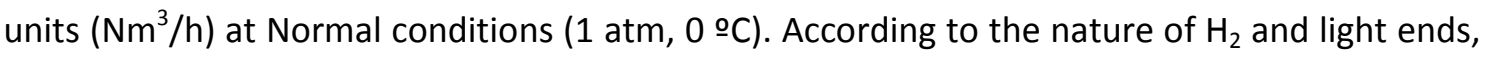


with compressibility factors close to 1 for a broad range of low pressures like the ones in the $\mathrm{H}_{2}$ network, the ideal gas model can be accepted without error at Normal conditions. Therefore, flow rates measurements at Normal conditions will be used as synonym of molar flow rates, implying the ideal gas model assumption.

A complete characterization of a gas stream $i$ is thus performed with the following three independent variables or degrees of freedom:

- Gas flow rate at Normal conditions, equivalent to molar flow rate: $\boldsymbol{F}_{i}\left(\mathrm{Nm}^{3} / \mathrm{h}\right)$

- Hydrogen purity: $\quad \boldsymbol{y}_{i}^{{ }^{\mathrm{H2}}}$ (\%1 mol)

- Molecular weight for light ends: $\boldsymbol{w}_{i}^{L / G}(\mathrm{~kg} / \mathrm{kmol})$

The stationary model of mass balances in nodes of gas streams comprises the following equations, with $22.415 \mathrm{Nm}^{3} / \mathrm{kmol}$ a constant arising from the ideal gas model regarding the equivalence of cubic meters at Normal conditions and molar flow rate:

- Total molar flow rate balance, equivalent to volumetric balance at Normal conditions with the ideal gas assumption, (eq. 1);

- Hydrogen molar flow rate balance, equivalent to hydrogen volumetric balance at Normal conditions with the ideal gas assumption, (eq. 2);

- Total mass flow rate balance, (eq. 3);

Additionally, the following equations apply for every stream $i$, with $2.01588 \mathrm{~kg} / \mathrm{kmol}$ the $\mathrm{H}_{2}$ molecular weight $w^{\text {H2 }}$ :

- Relation for the stream light ends purity $y_{i}^{L / G}$, (eq. 4);

- Relation for the stream molecular weight $w_{i}$ (eq. 5);

$$
\begin{gathered}
\sum_{i}^{I N} F_{i}=\sum_{j}^{\text {OUT }} F_{j} \quad \forall \text { node } \\
\sum_{i}^{I N}\left(F_{i} \cdot y_{i}{ }^{H 2}\right)=y_{j}{ }^{H 2} \cdot \sum_{j}^{\text {OUT }} F_{j} \quad \forall \text { node } \\
\sum_{i}^{I N} w_{i} \cdot\left(F_{i} / 22.415\right)=w_{j} \cdot \sum_{j}^{\text {OUT }}\left(F_{j} / 22.415\right) \quad \forall \text { node } \\
1=y_{i}{ }^{H 2}+y_{i}{ }^{L I G} \\
w_{i}=w^{H 2} \cdot y_{i}{ }^{H 2}+w_{i}^{L I G} \cdot\left(1-y_{i}{ }^{H 2}\right) \\
\end{gathered}
$$

No correlation between $w_{i}^{\mathrm{LG}}$ and $y_{i}^{H 2}$ has been observed (Fig. 4.1) for any gas streams $i$ either in the HP or MP/LP systems, according to all the laboratory composition analysis available along 5 years. Both variables are correlated when comparing values depending on the separator pressure, that is, whether the gas stream comes from the HP, the MP or the LP separator. However, regarding historical laboratory data for a single gas stream, no correlation occurs for 
the majority of the data. This fact can be justified because the composition of streams is not related to a single phenomenon but to different sources: gas composition depends on the reactions (where $\mathrm{H}_{2}$ consumption and composition of the light ends generated could be correlated), as well as on the quality of the make-up sources. Moreover, the operating range for $y_{i}^{H 2}$ is quite narrow, especially in the HP system, whereas $w_{i}^{L G}$ is influenced by considerable process variability, thus reinforcing the random effect.

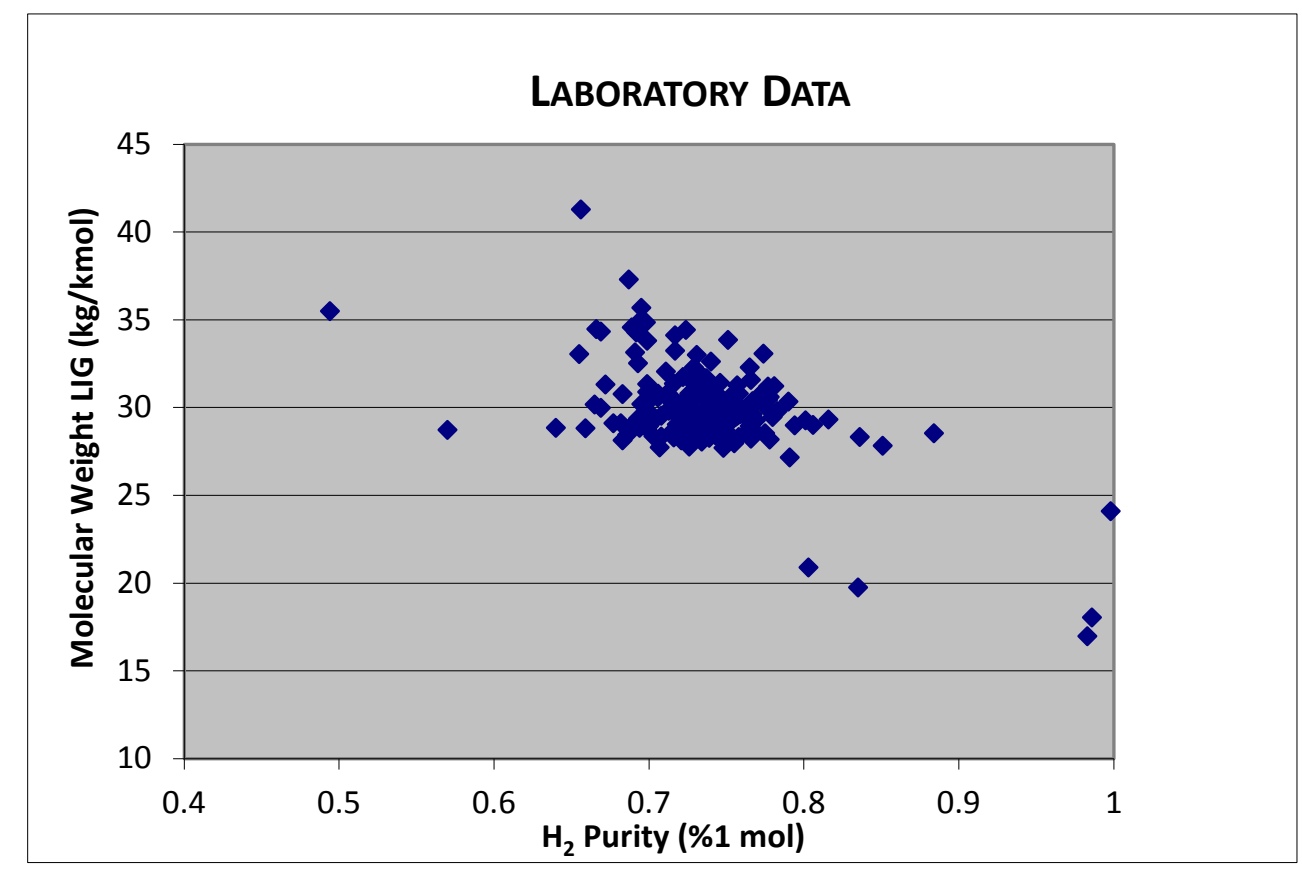

Figure 4.1. No correlation can be assumed for $w_{i}^{L / G}$ and $y_{i}^{H 2}$ in a general stream $i$ according to historical laboratory data.

At the global network scope, it should be noticed according to the equations that the subsystem regarding the total molar flow rate balance (eq. 1) for all gas streams and unit operations can be decoupled and solved independently of the rest of variables characterizing gas streams, being a linear system. In an analogous way, the subsystem comprising both the total molar flow rate balance and the $\mathrm{H}_{2}$ molar flow rate balance (eq. 1, 2) for all gas streams and unit operations enables to determine all the $\mathrm{H}_{2}$ purities irrespective of the molecular weights. Finally, the solution of the system comprising the three equations (eq. 1-3) including the total mass flow rate balance, depends on all the molar flow rates and $\mathrm{H}_{2}$ purities already computed.

\subsection{2- Reactor Model}

Reactor in consumer plants is typically of the fixed-bed catalytic type, with two/one-phase flow being the hydrocarbon feed partially or totally converted into vapour in the upstream heatexchangers and in some cases furnaces. Quench streams reach the space between the reactor 
beds with the purpose of temperature control for the exothermic reactions. These quench streams can be either recycled gas from the HP separator or recycled liquid from the HP separator liquid outlet.

According to the aforementioned mass balances model, 3 independent parameters have been formulated to account for the consumption of $\mathrm{H}_{2}$ and the generation of light ends in the gas phase due to both the hydrotreatment and side reactions. The independent parameters are:

- $\mathrm{H}_{2}$ consumption in the gas phase: $\quad \Delta \boldsymbol{R}_{\mathrm{H}_{2}}\left(\mathrm{Nm}^{3} \mathrm{H}_{2} / \mathrm{h}\right)$

- Molar light ends generation in the gas phase: $\quad \Delta \boldsymbol{R}_{\text {LIG }}\left(\mathrm{Nm}^{3} \mathrm{LIG} / \mathrm{h}\right)$

- Average molecular weight for the light ends generated in the gas phase, equivalent to the mass generation of light ends: $\quad \Delta \boldsymbol{R}_{w L G G}\left(\mathrm{~kg}^{\mathrm{LG}} / \mathrm{kmol}^{\mathrm{LG}}\right) \quad\left[\Delta \boldsymbol{R}_{w}(\mathrm{~kg} \mathrm{LIG} / \mathrm{h})\right]$

Using the nomenclature shown in Fig. 4.2, corresponding to the simplified flowsheet diagram for a general $\mathrm{H}_{2}$ consumer plant, reactor model for the gas phase is formulated according to the total molar flow rate balance (eq. 6), hydrogen molar flow rate balance (eq. 7), and total mass flow rate balance (eq. 8):

$$
\begin{aligned}
& F_{I N}=F_{\text {OUT }}+\Delta R_{H 2}-\Delta R_{L I G} \\
& F_{I N} \cdot y_{I N}{ }^{H 2}=F_{\text {OUT }} \cdot y_{\text {OUT }}{ }^{H 2}+\Delta R_{H 2} \\
& \left(\frac{F_{I N}}{22.415}\right) \cdot w_{I N}=\left(\frac{F_{\text {OUT }}}{22.415}\right) \cdot w_{\text {OUT }}+\left(\frac{\Delta R_{H 2}}{22.415}\right) \cdot 2.01588-\Delta R_{w}
\end{aligned}
$$

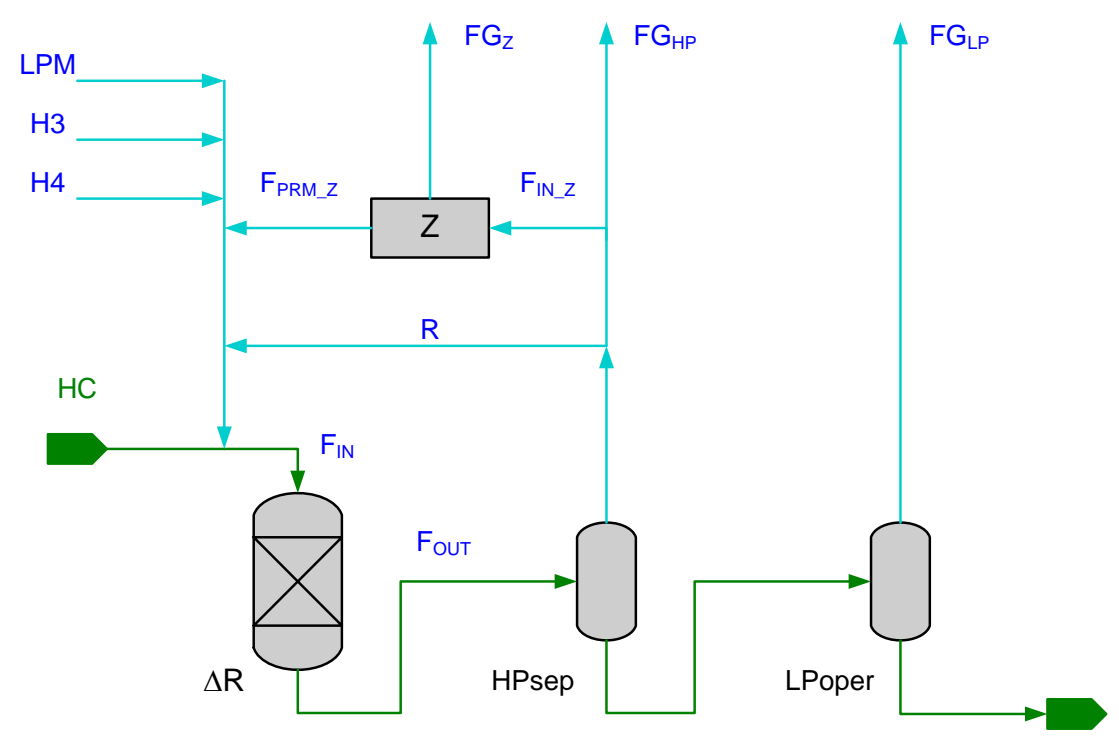

Figure 4.2. Simplified flowsheet diagram for an $\mathrm{H}_{2}$ consumer plant. 
Additionally, reactor model comprises the mass balance for the liquid phase (eq. 9) with $W_{H C}$ $(\mathrm{kg} \mathrm{HC} / \mathrm{h}$ ) the hydrocarbon mass flow rate, and the assumptions that the hydrocarbon density $\left(\rho_{H C}\right)$ and molecular weight $\left(w^{H C}\right)$ maintain their values at the reactor outlet equal to those at the inlet (eq. 10,11), in spite of the reactions. Although in industrial practice the volumetric yield of the liquid hydrocarbon is slightly increased in the reactor, with the subsequent decrease in the hydrocarbon density, the computed hydrocarbon volumetric flow rates $F_{H C}\left(\mathrm{~m}^{3}\right.$ $\mathrm{HC} / \mathrm{h}$ ) are only employed in the computation of the solubility equilibrium in separators, therefore the assumption results in a negligible inconsistency between the specific consumption/generation terms (per hydrocarbon processed) and the separators solubility parameters.

$$
\begin{aligned}
& W_{H C, I N}=W_{H C, \text { OUT }}-\left(\frac{\Delta R_{H 2}}{22.415}\right) \cdot 2.01588+\Delta R_{w} \\
& \rho_{H C, I N}=\rho_{H C, \text { OUT }} \\
& w^{H C}{ }_{I N}=w^{H C} \text { OUT }
\end{aligned}
$$

An approximation has been made in order to avoid non-linear constraints in the subsystem comprising all the total molar flow rate balances (equivalent to volumetric balances), i.e. in order to preserve linearity in constraints regarding flow rate for all gas streams. Both the $\mathrm{H}_{2}$ consumption mass rate $(2.01588 / 22.415) \cdot \Delta R_{\mathrm{H}_{2}}\left(\mathrm{~kg} \mathrm{H}_{2} / \mathrm{h}\right)$ and the light ends generation mass rate $\Delta R_{w}(\mathrm{~kg} \mathrm{LIG} / \mathrm{h})$ are negligible with respect to the processed hydrocarbon mass flow rate $W_{H C}(\mathrm{~kg} \mathrm{HC} / \mathrm{h})$, being about $1 \%$ for all the consumer plants. Besides, the sign for $\Delta R_{H 2}$ and $\Delta R_{w}$ is the opposite being similar in magnitude, thus counterbalancing each other and resulting in an expected error of less than $1 \%$. Therefore, eq. 9 is substituted by eq. 12 , and consequently hydrocarbon volumetric flow rates $F_{H C}\left(\mathrm{~m}^{3} \mathrm{HC} / \mathrm{h}\right)$ at reactor inlet and outlet are equal.

$$
W_{H C, I N}=W_{H C, O U T}
$$

All total molar flow rate balances for gas streams are therefore modelled with a linear subsystem of equations, which can be solved in advance and independently of individual material balances which are non-linear. This feature has been used to simplify the mathematical optimization problems formulated to solve the data reconciliation and optimal redistribution issues.

Other relations among variables are stated below (eq. 13-16), corresponding to the definition of the average molecular weight for the light ends generated $\Delta R_{\text {wLIG }}(\mathrm{kg} \mathrm{LIG} / \mathrm{kmol} \mathrm{LIG),} \mathrm{the}$ definition of the ratio of light ends generation to $\mathrm{H}_{2}$ consumption $\Delta R_{\text {LIGH2 }}\left(\mathrm{Nm}^{3} \mathrm{LIG} / \mathrm{Nm}^{3} \mathrm{H}_{2}\right)$, as well as the volumetric hydrocarbon flow rate $F_{H C}\left(\mathrm{~m}^{3} \mathrm{HC} / \mathrm{h}\right)$ and molar hydrocarbon flow rate $M_{H C}(\mathrm{kmol} \mathrm{HC} / \mathrm{h})$, being $\rho_{H C}\left(\mathrm{~kg} \mathrm{HC} / \mathrm{m}^{3} \mathrm{HC}\right)$ the hydrocarbon density and $w^{H C}(\mathrm{~kg} \mathrm{HC} / \mathrm{kmol} \mathrm{HC})$ the hydrocarbon molecular weight:

$$
\Delta R_{w L I G}=\left(\frac{\Delta R_{w}}{\Delta R_{L I G} / 22.415}\right)
$$




$$
\begin{aligned}
& \Delta R_{L I G H 2}=\left(\frac{\Delta R_{L I G}}{\Delta R_{H 2}}\right) \\
& F_{H C}=W_{H C} / \rho_{H C} \\
& M_{H C}=W_{H C} / w^{H C}
\end{aligned}
$$

Another important variable refers to the $\mathrm{H}_{2} / \mathrm{HC}$ ratio at reactor inlet, defined with eq. 17 .

$$
r_{H 2_{-} H C}=\frac{F_{R E A C T_{-} I N} \cdot y_{\text {REACT_IN }}{ }^{H 2}}{F_{\text {REACT_IN, HC }}}
$$

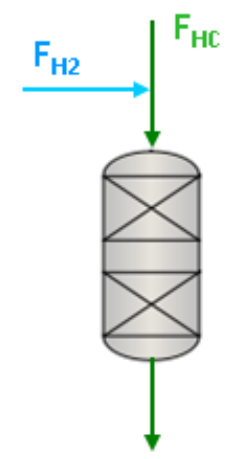

Specific terms per hydrocarbon processed for the $\mathrm{H}_{2}$ consumption $\left(\Delta R_{\mathrm{H} 2}{ }^{\mathrm{sp}}, \mathrm{Nm}^{3} \mathrm{H}_{2} / \mathrm{m}^{3} \mathrm{HC}\right)$ and light ends generation $\left(\Delta R_{L I G}{ }^{s p}, \mathrm{Nm}^{3} \mathrm{LIG} / \mathrm{m}^{3} \mathrm{HC}\right)$ are also defined for comparison purposes, eq. $18,19$.

$$
\begin{aligned}
& \Delta R_{H 2}^{s p}=\Delta R_{H 2} / F_{H C, I N} \\
& \Delta R_{L I G}{ }^{s p}=\Delta R_{L I G} / F_{H C, I N}
\end{aligned}
$$

From the point of view of parameter comprehension, certain remarks should be done:

- Specific $\mathrm{H}_{2}$ consumption per hydrocarbon processed $\Delta R_{\mathrm{H} 2}{ }^{s p}$ is determined from the data reconciliation problem according to on-line measurements from both flow meters and analysers; model sensitivity to measured variables and available redundancy enable to guarantee a reliable estimation.

- For each consumer plant, narrow bounded ratios are provided for the parameters or intensive variables $\Delta R_{w L I G}$ and $\Delta R_{L I G H 2}$, according to process knowledge and design data. Intensive variables are easier to delimit; furthermore an appropriate range is mandatory to achieve a good and consistent estimation of $\mathrm{H}_{2}$ consumption, otherwise the problem is overparameterized.

Regarding the ratio of light ends generation to $\mathrm{H}_{2}$ consumption $\Delta R_{L I G H 2}$, even though the ratio of light ends generation is small as compared to that for $\mathrm{H}_{2}$ consumption, ranging $\Delta R_{L G G 2}$ from 0.01-0.07 approximately, its absolute value for 
big consumer plants can be significant at the network scope. Only in the case of the catalytic-reforming producer plants the ratio is important, ranging from 0.200.40 , and can be accurately estimated because: i) these plants do not have makeup from alternative sources and are only linked to the network in one direction (they are producer plants); ii) an analyser for $\mathrm{H}_{2}$ purity is available in the HP system.

\subsection{3- Membranes Model}

The membranes unit consist of several packages of fabric chambers that can be put into operation independently. After being heated, and at the expense of a certain pressure drop, $\mathrm{H}_{2}$ and light molecules pass preferentially across the fabric constituting the permeate stream, whereas the remaining gas unable to cross the pores constitutes the purge stream, which is usually sent to the fuel gas FG header. The membranes unit is not of the pressure swing adsorption PSA type, which is capable of a higher selectivity providing $\mathrm{H}_{2}$ of approximately $1 \%$ (\%1 mole) purity; PSA units are available in high-purity producer plants (steam-reforming furnaces).

Cost savings due to $\mathrm{H}_{2}$ make-up decrease are always higher than membranes operating costs, which are not significant because only low-pressure steam is needed to increase gas temperature up to around 80 ㅇ․ Usually membranes operating costs can be disregarded.

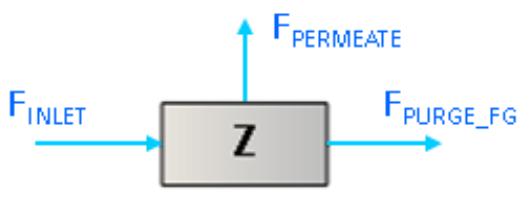

In an analogous way, the membranes model comprises the 3 total mass balances: total molar flow rate balance (eq. 20), hydrogen molar flow rate balance (eq. 21), and total mass flow rate balance (eq. 22). Since there are two outlet gas streams in this unit, 6 variables have to be computed by means of the model in this unit.

$$
\begin{aligned}
& F_{I N_{-} Z}=F_{P R M_{-} Z}+F_{F G_{-} Z} \\
& F_{I N_{-} Z} \cdot y_{I N_{-} Z}{ }^{H 2}=F_{P R M_{-} Z} \cdot y_{P R M_{-} Z}{ }^{2}+F_{F G_{-} Z} \cdot y_{F G_{-} Z}{ }^{22} \\
& \left(\frac{F_{I N_{-} Z}}{22.415}\right) \cdot w_{I N_{-} Z}=\left(\frac{F_{P R M_{-} Z}}{22.415}\right) \cdot w_{P R M_{-} Z}+\left(\frac{F_{F G_{-} Z}}{22.415}\right) \cdot w_{F G_{-} Z}
\end{aligned}
$$

The membranes system is additionally constituted by two equations modelling the distribution of $\mathrm{H}_{2}$ and light ends between the permeate and the purge streams, eq. 23, 24.

$$
\begin{aligned}
& y_{P R M_{-} Z}{ }^{H 2}=a \cdot\left(F_{F G_{-} Z} / F_{I N_{-} Z}\right)+b \cdot y_{I N_{-} Z}{ }^{H 2}+c \\
& w_{P R M_{-} Z}{ }^{L I G}=w_{I N_{-} Z}{ }^{L I G}
\end{aligned}
$$


A simple modelling equation for the prediction of the $\mathrm{H}_{2}$ purity in the permeate stream has been formulated, as a function of both the ratio purged to fuel gas with respect to the inlet flow rate, and the $\mathrm{H}_{2}$ purity of the inlet stream. It is based on Petronor ${ }^{\circledR}$ staff process knowledge, with a rule of thumb according to which permeate $\mathrm{H}_{2}$ purity can be obtained by adding a bias to inlet $\mathrm{H}_{2}$ purity. The proposed model is consistent with experimental evidence: i) the permeate $\mathrm{H}_{2}$ purity increases with the inlet $\mathrm{H}_{2}$ purity; ii) it is expected that selectivity of the separation increases with higher purge flow rate, that is, the lower the permeate flow rate, the higher the permeate $\mathrm{H}_{2}$ purity. Parameters for the linear regression were estimated offline with historical data, and included as fixed model coefficients. A linear experimental correlation was accurate enough according to validation results.

The second modelling equation determines the distribution of the different light ends between the permeate and the purge streams, according to their different molecular weights. It has been supposed that an even distribution takes place, irrespective of the different molecular weights. This is justified because in the HP system, to which the inlet to membranes belongs, the $w^{L / G}$ is usually within a narrow range, and has been further corroborated with laboratory historical data for the permeate and purge streams compositions.

Assuming that the inlet stream is completely specified, the membranes model target is to determine the streams in a downstream direction. Therefore, one independent variable or degree of freedom remains, both in the model and in the real operation, which is:
- Purge flow rate to Fuel Gas:
$\boldsymbol{F}_{\text {PURGE_FG }}\left(\mathrm{Nm}^{3} / \mathrm{h}\right)$

Therefore, the membranes operating point is fixed by manipulating the inlet and purge flow rates.

\subsection{4- Separator Model}

The separator model represents either a flash-drum single separator or a combination of flashdrums and distillation columns. Typically the HP separator is a single flash-drum whereas the MP and LP separators comprise several units operations, with a common measured (flow rate) outlet gas stream.

According to process knowledge, the gas solved in the liquid outlet from the HP separator is quite independent on $\mathrm{H}_{2}$ redistribution or gas inlet to the reactor. Pressure and temperature are the main variables influencing separation. Pressure is controlled at a fixed value, according to compressors and compression ratios design, as well as design conditions. Temperature is regulated at the minimum possible value achieved with air cooled heat exchangers, thus enabling the best relative separation of $\mathrm{H}_{2}$ from light ends. 


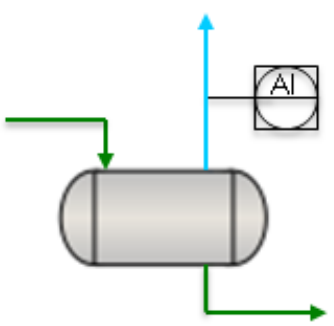

With analogy to other units, the separator model consists of the 3 total mass balances: total molar flow rate balance (eq. 25), hydrogen molar flow rate balance (eq. 26), and total mass flow rate balance (eq. 27). According to the pressure and temperature in the operation, and also depending on the hydrocarbon composition, part of the gas stays solved in the liquid outlet whereas the remaining gas is separated in an outlet gas stream; the residence time is assumed enough to reach equilibrium. Consequently, a solubility equilibrium model is formulated consisting of three equations; no degrees of freedom or manipulated variables exist in the real operation regarding the gas outlet stream, since it is separated under pressure control (mass balance fulfilment).

Different authors have proposed models for $\mathrm{H}_{2}$ and light gases solubility equilibrium in petroleum fractions. Riazi (2007) establishes equations depending on the hydrocarbon nature, i.e. the content of paraffinic, naphthenic and aromatic compounds. Moysan et al. (1983) on the contrary proposes a model based on activity coefficients and equations of state, also needing compositions for all the involved gas species. A simplified approach has been considered for the modelling of the solubility relations due to the lack of on-line measurements for composition and the significant process variability.

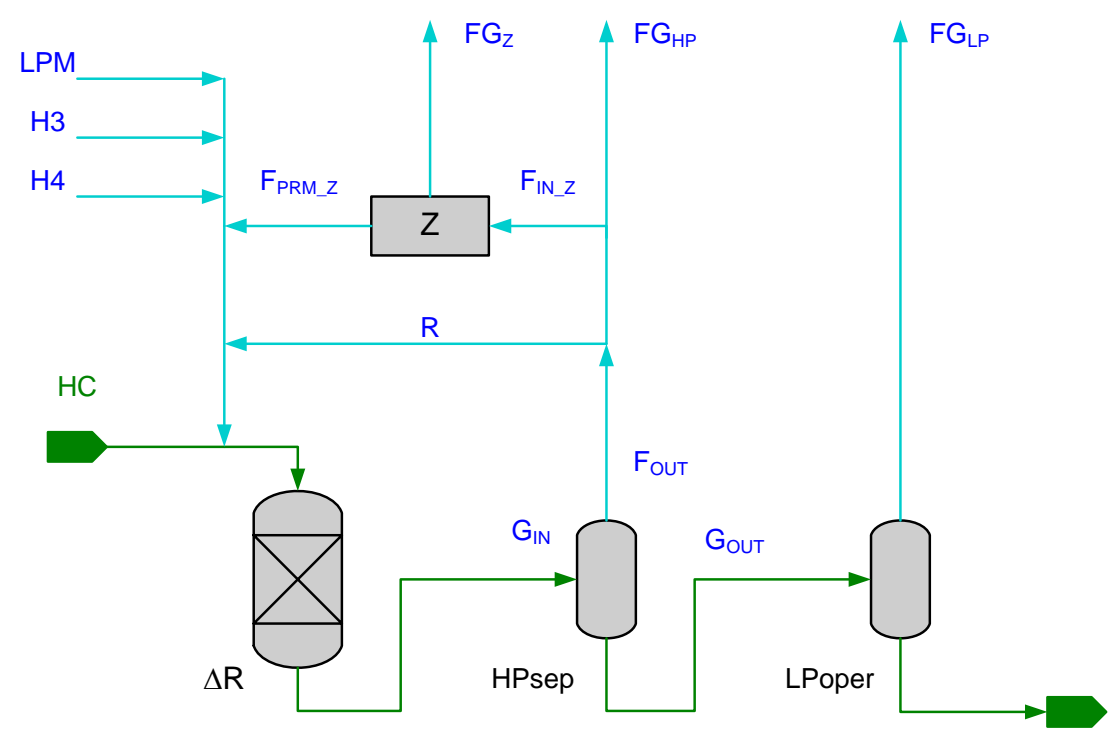

Figure 4.3. Simplified flowsheet diagram for an $\mathrm{H}_{2}$ consumer plant. 
Using the nomenclature shown in Fig. 4.3 , and being $x_{I N}, x_{O U T}$ the molar compositions (\%1 mol) and $G_{I N}, G_{\text {OUT }}$ the molar flow rates $(\mathrm{kmol} / \mathrm{h})$ of the inlet and outlet liquid streams, comprising both the liquid hydrocarbon and the gas either solved or in a two-phase mixture, the three equations modelling the material balances (eq. 25-27) for gas are stated below.

$$
\begin{aligned}
& G_{I N} \cdot\left(1-x_{I N}{ }^{H C}\right)=\left(\frac{F_{\text {OUT }}}{22.415}\right)+G_{\text {OUT }} \cdot\left(1-x_{\text {OUT }}{ }^{H C}\right) \\
& G_{I N} \cdot x_{I N}{ }^{H 2}=\left(\frac{F_{\text {OUT }}}{22.415}\right) \cdot y_{\text {OUT }}{ }^{H 2}+G_{\text {OUT }} \cdot x_{\text {OUT }}{ }^{H 2} \\
& G_{I N} \cdot\left(1-x_{I N}{ }^{H C}\right) \cdot w_{G I N}=\left(\frac{F_{\text {OUT }}}{22.415}\right) \cdot w_{\text {FOUT }}+G_{\text {OUT }} \cdot\left(1-x_{\text {OUT }}{ }^{H C}\right) \cdot w_{\text {GOUT }}
\end{aligned}
$$

The hydrocarbon component stays invariable, and is modelled with the corresponding mass balance (eq. 28) and the corresponding assumptions regarding density and molecular weight (eq. 29, 30).

$$
\begin{aligned}
& G_{I N} \cdot x_{I N}{ }^{H C}=G_{\text {OUT }} \cdot x_{\text {OUT }}{ }^{H C} \\
& \rho_{H C, I N}=\rho_{H C, \text { OUT }} \\
& w^{H C}{ }_{\text {IN }}=w^{H C} \text { OUT }
\end{aligned}
$$

Equations modelling the solubility equilibrium in the separator (eq. 31-33) are formulated by defining three solubility parameters. The three independent parameters are:

- Total gas solubility in the liquid hydrocarbon: $\quad \boldsymbol{k}_{\text {gasHc }}^{\mathrm{s}}\left(\mathrm{Nm}^{3} \mathrm{Gas} / \mathrm{m}^{3} \mathrm{HC}\right)$

- Relative distribution coefficient for $\mathrm{H}_{2}$ and light ends between the gas and liquid phases:

$$
\boldsymbol{k}_{\text {aH2LIG () }}^{s}
$$

- Relative distribution coefficient for light ends according to their molecular weight, between the gas and liquid phases: $\quad \boldsymbol{k}_{\text {wLIG }}^{S}\left(\mathrm{~kg} \cdot \mathrm{kmol}^{-1} / \mathrm{kg} \cdot \mathrm{kmol}^{-1}\right)$

with $G_{\text {OUT }}(\mathrm{kmol} / \mathrm{h})$ the total molar flow rate for both gas and hydrocarbon in the liquid outlet stream, $y_{i}{ }^{j}$ and $x_{i}{ }^{j}(\% 1 \mathrm{~mol})$ the molar compositions for component $j$ in the outlet gas $F_{\text {OUT }}$ and outlet liquid $G_{\text {OUT }}$ streams respectively, and $w_{i}{ }^{j}(\mathrm{~kg} / \mathrm{kmol})$ the molecular weight for component $j$ in stream $i$.

$$
\begin{array}{ll}
k_{\text {gasHC }}^{S}=\frac{22.415 \cdot G_{\text {OUT }} \cdot\left(x_{\text {Gout }}^{H 2}+x_{\text {Gout }}^{L I G}\right)}{G_{\text {OUT }} \cdot x_{\text {Gout }}^{H C} \cdot\left(w_{H C} / \rho_{H C}\right)} & k_{\text {gasHC }}^{S}>0 \\
k^{S}{ }_{\alpha H 2 L I G}=\frac{y_{\text {Fout }}^{H 2} / x_{\text {Gout }}^{H 2}}{y_{\text {Fout }}^{L I G} / x_{\text {Gout }}^{L I G}} & k^{S}{ }_{\alpha H 2 L I G}>1 \\
k^{S}{ }_{\text {wLIG }}=\frac{w_{\text {Fout }}^{L I G}}{w_{\text {Gout }}^{L I G}} & k^{S}{ }_{\text {wLIG }}<1
\end{array}
$$


The parameter $k_{\text {gasHc }}^{S}$ accounts for gas solubility in liquid hydrocarbon entailing that the hydrocarbon is saturated in gas, which is consistent with experience in real operation as aforementioned. This parameter multiplied by the hydrocarbon flow rate determines the total gas that will be separated downstream the corresponding separator. The total gas solved in the liquid hydrocarbon is one of the variables to which the separator model is more sensitive, and presents the advantage of being measured on-line.

The parameters $k_{\alpha H 2 L G G}^{S}$ and $k_{w L I G}^{S}$ are necessarily above or below 1 as aforementioned (eq. 32 , 33), due to the same reason: lighter components are prone to go to the gas phase while heavier components by comparison stay preferentially in the liquid phase. As the nature of light ends does not change significantly, and operating conditions of pressure, temperature, $\mathrm{H}_{2}$ purity and hydrocarbon quality in separators vary in a limited range, model parameters $k_{\text {aHzLIG }}^{S}$ and $k_{W L I G}^{S}$ can be bounded to a certain narrow range. A significant change in the nature of light ends could be due to a different source for light ends where methane $\mathrm{CH}_{4}$ was nearly the only impurity thus affecting the relative solubility. Since in this case all impurities are generated mainly in the catalytic-reforming producer plants and other consumer plants, and irrespective of inescapable process variability, always $\mathrm{C}_{2} \mathrm{H}_{6}, \mathrm{C}_{3} \mathrm{H}_{8}$ and $\mathrm{C}_{4}$ will be present to a certain extent, it is accepted that the relative solubility will not be too much influenced. Furthermore, both $k^{S}{ }_{\alpha H 2 L I G}$ and $k^{S}{ }_{\text {wLIG }}$ have been defined as relative solubility coefficients, analogous to the relative volatility in the vapour-liquid equilibrium, being in general relative coefficients more stable and undergoing smaller changes in the presence of process variability.

Temperature in HP separators is controlled with aerial cooler heat exchangers, thus with temperatures ranging from about 10 to $40 \stackrel{\circ}{\mathrm{C}}$ in nearly all cases. Solubility parameters have been specified as a function of operating pressure and hydrocarbon cut, and comparisons among different plants can be performed being in agreement.

As the number of model degrees of freedom is higher than the available number of on-line measurements, particularly when considering uncertainties in those measurements, it is not justified to increase solubility model complexity. The proposed model provides various interesting features and encompasses the following behaviours:

- Although it is simple and not rigorous, nevertheless it is consistent and physically meaningful, and can be calibrated on-line for the variable to which the greatest sensitivity is shown: the highest sensitivity corresponds to the solubility of gas in liquid hydrocarbon, i.e. the total gas flow rate separated downstream the HP separator in MP/LP operations.

- Proposed model for separators is congruent with experimental evidence, while guaranteeing mathematical consistency for all variables.

Provided that the allowed range for model parameters fulfils the aforementioned constraints (eq. 31-33), then the coefficients determine the distribution of components $\left(\mathrm{H}_{2}\right.$ and light ends) between the gas and liquid phases, therefore, referring always to gas in exempt base of liquid, it is always assured that: i) $\mathrm{H}_{2}$ 
purity in gas will be higher than the inlet one, and $\mathrm{H}_{2}$ purity in liquid will be lower than the inlet one; ii) light ends molecular weight in gas will be lower than in the inlet, and light ends molecular weight in liquid will be higher than in the inlet.

$\mathrm{H}_{2}$ purity $y^{H 2}$ related for $\mathrm{HP}, \mathrm{MP}$ and LP separators, with a monotonous dependence: the higher the $y^{H 2}$ in the HP separator, the higher the $y^{H 2}$ in the MP one, and also in the LP one.

Independence between the $y^{H 2}$ and the $w^{L / G}$ in all separators, supported by experimental laboratory data.

- Model flexibility is enough to fit the desired range of compositions for all separators in the network, according to the available laboratory historical data:

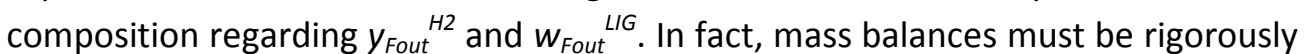
fulfilled, and the quotients that define the solubility coefficients will have a certain value in any case. Lack of flexibility will only arise in case of a narrow range for parameter values. Moreover, both $y^{\text {H2 }}$ and $w$ for a separator gas outlet are very influenced by the gas flow rate, therefore a poor fitting in these variables can be justified not only because of the lack of model structural flexibility according to the specified ranges for parameters or a bad parameter tuning, but also because of flow measurement error and uncertainty.

- As opposed to other solubility equilibrium models in the literature (Riazi, 2007), (Moisan, ), it does not rely on hydrocarbon composition and/or individual light ends composition, which are not measured on-line.

- Only in the HP system on-line $\mathrm{H}_{2}$ purity analysers exist, whereas downstream in the gas separated in the MP and LP separations no quality on-line measurements are available. Consequently, a model is needed to determine gas streams composition. The easiest choice would be to fix average historical data of $y^{\text {H2 }}$ and $w^{L G}$ for all gas streams separated in the MP/LP separators, which is an approach that can provide reasonable results although without accounting for process variability. The aforementioned model is physically consistent and meaningful, while simultaneously allowing for certain variability.

Regarding practical implementation in the EcosimPro ${ }^{\circledR}$ environment and numerical computational issues, the modelling equations have been manipulated in order to facilitate convergence. The non-linear algebraic loop resulting from the separator system in the steadystate computation is substituted by a second order equation. The $\mathrm{H}_{2}$ molar flow rate balance and the equation for the relative solubility of $\mathrm{H}_{2}$ and light ends defined by parameter $k_{\alpha{ }_{\alpha 2 L G}}$ are rearranged, and the resulting second order equation is solved for $\mathrm{H}_{2}$ purity in the gas outlet stream $y_{\text {Fout }}{ }^{H 2}$, thus being explicitly computed. Provided that always a gas stream is separated, i.e. $F_{O U T}$ is positive, the same root of the equation is always the correct solution. 


\subsection{5- Low-Pressure Separator Model}

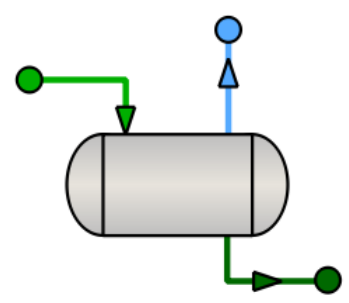

Its purpose is the modelling of the complete separation of the solved gas from a liquid hydrocarbon stream. Therefore, the model comprises the corresponding equations to force the equivalence of the 3 independent variables characterizing gas streams $\left(F, y^{H 2}, w^{L / G}\right)$, as well as the equations to force the equivalence of the 3 independent variables characterizing hydrocarbon streams $\left(F_{H C}, \rho_{H C}, w^{H C}\right)$, between the inlet and outlet streams respectively.

\subsection{6- Model of Compressors, Mixers, Splitters, Valves, Pipes, Meters, Tanks}

The model for the rest of the unit operations is very simple and is straightforwardly obtained from the well-known material balances. Certain particular matters regarding the implementation will be remarked at the end of this chapter under the epigraph for EcosimPro ${ }^{\circledR}$ environment.

\section{Compressors}

Compressors, either of the centrifugal or the reciprocating type, are provided with the feature of upper/lower capacity limit configuration.
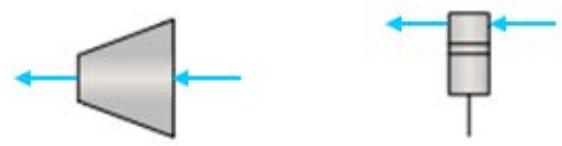

\section{Flowmeters, analysers and pipes}

Flowmeters, analisers and pipes are useful to configure upper/lower limits regarding capacities and constraints. Additionally, flowmeters and analisers are provided with the necessary configuration parameters and variables to aid in the data adquisition process and the link with measured variables in the Petronor ${ }^{\circledR}$ SCADA.

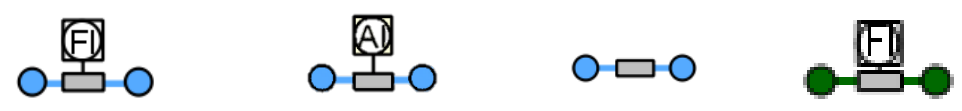

In the initialization step of the simulation, measured values are assigned to the corresponding variables in the meter components. In the case of hydrocarbon meters, only the necessary and 
sufficient number has been used, disregarding redundant hydrocarbon measurements when available; those more reliable or providing more information like the mass compensated measurement (together with the flow compensated measurement) were preferred. Default values for hydrocarbon density were employed in the absence of mass measurement.

The "tub" or pipe component is used for different purposes when no flow meter is available in a particular stream: specify a valve tag to read the opening from the SCADA, specify certain upper/lower bounds for flow rate, or specify other characteristics of the stream regarding problem definition.

\section{Sources - Sinks}

The model is very simple and is straightforwardly obtained from the well-known material balances.
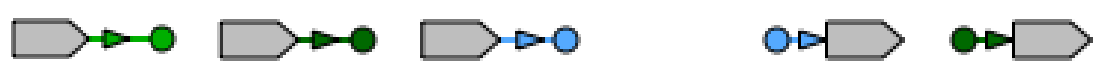

\section{Tank}

The tank unit enables the modelling of drums where liquid hydrocarbon is not saturated in gas and consequently, it is mixed with an inlet gas stream under pressure control. Usually it corresponds to feedstock tanks upstream in the consumer plants flowsheet, especially in the case of plants processing light feedstock (butane/butene), where solubility of light ends is high.

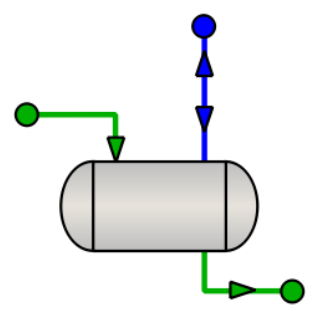

Model comprises the three equations for the material balances as well as the three relations for the solubility model. Either an inlet or an outlet gas stream is allowed, fixed under pressure control according to a split-range control structure. When the gas stream is an inlet one, both $y^{H 2}$ and $w^{L / G}$ in the gas stream are determined upstream, and the three material balances together with the coefficient $k_{\text {gasHC }}^{S}$ are sufficient to compute the solved gas in the liquid outlet. When the gas stream is an outlet one, the model is equivalent to the HP separator one, involving the three solubility relations.

$$
\begin{aligned}
& F_{\text {GIN }}=F_{\text {OUT }}+F_{\text {GOUT }} \\
& F_{\text {GIN }} \cdot y_{\text {GIN }}{ }^{H 2}=F_{\text {OUT }} \cdot y_{\text {OUT }}{ }^{H 2}+F_{\text {GOUT }} \cdot y_{\text {GOUT }}{ }^{H 2} \\
& \left(\frac{F_{\text {GIN }}}{22.415}\right) \cdot w_{\text {GIN }}=\left(\frac{F_{\text {OUT }}}{22.415}\right) \cdot w_{\text {FOUT }}+\left(\frac{F_{\text {GOUT }}}{22.415}\right) \cdot w_{\text {GOUT }}
\end{aligned}
$$




$$
\begin{gathered}
F_{H C, I N}=F_{H C, \text { OUT }} \\
\rho_{H C, I N}=\rho_{H C, \text { OUT }} \\
w_{I N}^{H C}=w^{H C} \text { OUT }
\end{gathered}
$$

with $F_{\text {OUT, }}, F_{\text {GIN }}, F_{\text {GOUT }}\left(\mathrm{Nm}^{3} / \mathrm{h}\right)$ the total gas volumetric flow rate at Normal conditions (equivalent to molar flow rate) for gas either alone or solved in the liquid hydrocarbon, $y_{i}{ }^{j}$ and $x_{i}{ }^{j}$ (\%1 mol) the molar compositions for component $j$ in the gas stream $i$ and mixture gas + hydrocarbon stream $j$ respectively, and $w_{i}{ }^{j}(\mathrm{~kg} / \mathrm{kmol})$ the molecular weight for component $j$ in gas stream $i$.

$$
\begin{aligned}
& k_{\text {gas HC }}^{S}=\frac{F_{\text {GOUT }}}{F_{\text {HC,OUT }}} \quad k_{\text {gasHC }}^{S}>0 \\
& k_{\alpha H 2 L I G}^{S}=\frac{y_{\text {Fout }}^{H 2} / x_{G o u t}^{H 2}}{y_{\text {Fout }}^{L I G} / x_{\text {Gout }}^{L I G}} \quad k_{\alpha H 2 L I G}^{S}>1 \\
& k^{S}{ }_{w L I G}=\frac{w_{\text {Fout }}^{L I G}}{w_{\text {Gout }}^{L I G}} \quad \quad k_{\text {wLIG }}^{S}<1
\end{aligned}
$$

\section{Mixers - Splitters for Gas streams}

Mixer and splitter components for gas streams have been modeled, either connecting pipes of only one allowed direction for flow or connecting pipes with two allowed directions for flow. They will be explained in detail in the epigraph for EcosimPro ${ }^{\circledR}$ implementation.
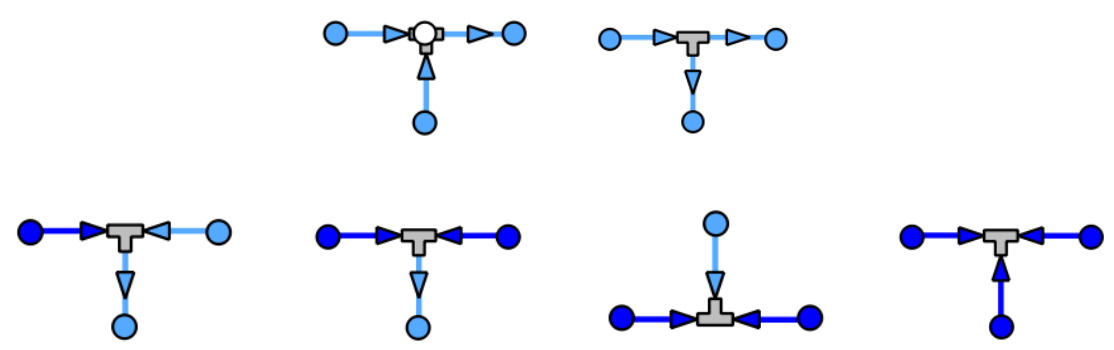

Although EcosimPro ${ }^{\circledR}$ provides the capability of parameterizing the number of ports of a certain type, however it has been preferred to model different components according to the different number of inlet and outlet ports, in order to strengthen graphical modeling visualization and considering that the number of needed combinations is small.

Mixers and splitters components for gas streams are modelled with the three well-known material balances; additionally in the case of splitters two more equations are added to determine the composition ( $y_{\text {OUT }}{ }^{H 2}$ and $w_{\text {OUT }}{ }^{L / G}$ ) for one of the outlet streams, i.e. the intensive variables. 


$$
\begin{aligned}
& y_{\text {OUT2 }}{ }^{H 2}=y_{I N}{ }^{H 2} \\
& w_{\text {OUT2 }}{ }^{\text {LIG }}=w_{I N}{ }^{L I G}
\end{aligned}
$$

\section{Mixers - Splitters for Hydrocarbon streams}

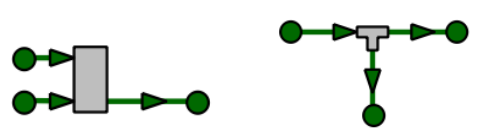

Mixers and splitters components for hydrocarbon streams are modelled with the total mass flow rate balance $\left(W_{i}, \mathrm{~kg} / \mathrm{h}\right)$, a weighted balance to compute the intensive variable $w^{H C}$, and the assumption of additive volumetric flow rates to estimate $\rho_{H C}$. Additionally, in the case of splitters two more equations are added, to determine the composition $\left(\rho_{H C}\right.$ and $\left.w^{H C}\right)$ for one of the outlet streams.

$$
\begin{aligned}
& \sum_{i}^{I N} W_{H C, i}=\sum_{j}^{O U T} W_{H C, j} \\
& \left(\frac{1}{W_{O U T}^{H C}}\right) \cdot \sum_{i}^{I N} W_{H C, i}=\sum_{i}^{I N}\left(\frac{W_{H C, i}}{W_{i}^{H C}}\right) \\
& \sum_{i}^{I N} F_{H C, i}=\sum_{j}^{O U T} F_{H C, j}
\end{aligned}
$$

The additional equations in the case of a hydrocarbon splitter component are:

$$
\begin{aligned}
& \rho_{\text {HC,OUT2 }}=\rho_{H C, I N} \\
& w_{\text {OUT2 }}{ }^{H C}=w_{I N}{ }^{H C}
\end{aligned}
$$

\section{Valves}

Valves of different types are modelled, with the purpose of assuring zero flow rate in a gas stream in certain conditions: i) in streams with only one allowed direction for flow; ii) in headers with two allowed directions for flow; iii) with two mutually exclusive inlets; iv) with two mutually exclusive outlets; v) with an inlet and outlet mutually exclusive connected to a pipe with two allowed directions.
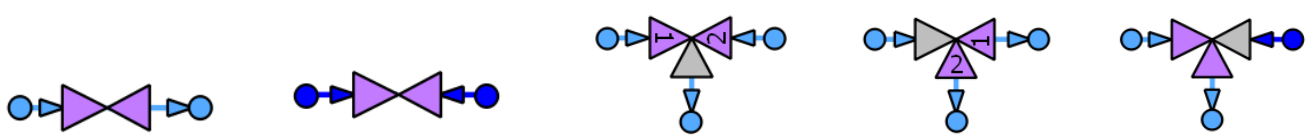

Valve models include logic expressions, because they enable to model exclusive relations between streams entailing structural changes in the network operation. Not always there is a 
correspondence of the valve model with a single physical element, being their purpose to easily establish allowed configurations for flow.

In order to have properly conditioned Jacobians and simulations with good convergence properties, all null flow rates have been forced to values in the order of magnitude of units. Although gas flow rates can vary in a broad range up to the order of $10^{4}$, the simulation is robust enough and supports nearly every combination of input step changes, provided that constraints assuring positive flow rates are fulfilled.

All valves have a Boolean variable that can be initialized to force either zero flow rate or not in the case of "dtOh2" and "dtOdir", the components to force zero flow rate in a pipe analogous to a manual valve. The pipe flow rate is assigned the value of the boundary variable named "ForceFbnd", which is meaningful whenever the flow rate is non-zero although just a dummy boundary whenever the valve is forced closed with zero flow rate. In the case of valves "v3v_2in" and "v3v_2out", the Boolean variable can be initialized to select one inlet or outlet between two exclusive ones, forcing the other one to zero. The zero-flow-rate state is forced in the initialization of the data reconciliation step, according to either valve openings readings when available on-line or either a combination of flow measurements and plant states.

"dtOh2" model

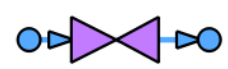

The model comprises the three well-known material balances, enabling to determine $F_{\text {OUT, }}$ ${ }_{\text {YUT }^{H 2}}{ }^{\mathrm{N}}, w_{\text {OUT }}{ }^{\mathrm{LG}}$, as well as the following additional eq. 48 enabling to force zero flow rate:

$$
\begin{array}{cc}
F_{I N}=\text { if }(\text { boolFZero }=\text { FALSE }) & F_{\text {ForceFbnd }} \\
\text { else } & 0
\end{array}
$$

\section{"v3v 2in" model}

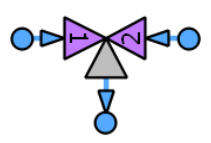

The model comprises the two well-known material balances for $\mathrm{H}_{2}$ molar flow rate and total mass flow rate, enabling to determine $y_{\text {OUT }}{ }^{H 2}$ and $w_{\text {OUT }}{ }^{L G G}$, as well as the following additional (eq. 49, eq. 50) enabling to force zero flow rate for one of the two exclusive inlets:

$$
\begin{aligned}
& F_{I N 1}=\text { if }(\text { boolForceIn1 }=\text { TRUE }) \quad F_{\text {OUT }} \\
& \text { else } \\
& 0 \\
& \begin{array}{cc}
F_{I N 2}=\text { if }(\text { boolForceIn } 1=\text { TRUE }) & 0 \\
\text { else } & F_{\text {OU }}
\end{array} \\
& F_{\text {OUT }}
\end{aligned}
$$


One manipulated variable or degree of freedom is lost by introducing this component, since one inlet flow rate is zero necessarily.

\section{"v3v 2out" model}

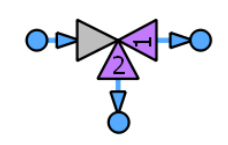

The model comprises the two well-known material balances for $\mathrm{H}_{2}$ molar flow rate and total

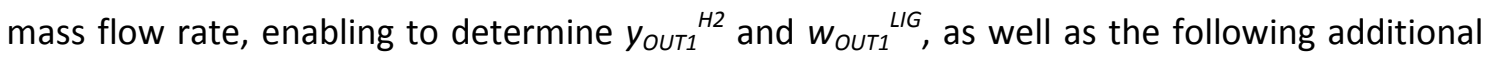
(eq. 51-53) enabling to force zero flow rate for one of the two exclusive outlets:

$$
\begin{aligned}
& F_{\text {OUT1 }}=\text { if }(\text { boolForceOut1 }=\text { TRUE }) \quad F_{\text {IN }} \\
& \text { else } 0 \\
& \left.F_{\text {OUT2 }}=\text { if (boolForceOut } 1=\text { TRUE }\right) \quad 0 \\
& \text { else } \quad F_{I N} \\
& y_{\text {OUT2 }}{ }^{H 2}=y_{\text {IN }}{ }^{H 2} \\
& w_{\text {OUT2 }}^{\text {LIG }}=w_{\text {IN }}{ }^{\text {LIG }}
\end{aligned}
$$

One manipulated variable or degree of freedom is lost by introducing this component, since one outlet flow rate is zero necessarily.

\section{"v3SR" model}

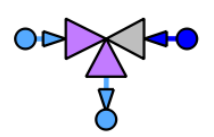

The "v3SR" component enables to model one inlet and one outlet streams mutually exclusive, typically in a split-range pressure control structure, where either gas enters or either gas comes out. The third stream $\left(F_{I N R}\right)$ necessarily has two allowed directions for flow.

The model comprises the two well-known material balances for $\mathrm{H}_{2}$ molar flow rate and total mass flow rate, enabling to determine $y_{\text {OUT }}{ }^{H 2}$ and $w_{\text {OUT }}{ }^{L I G}$, as well as the following additional (eq. 54,55 ) enabling to force zero flow rate for one of the two exclusive streams:

$$
\begin{array}{cc}
F_{I N}=i f(\text { boolForceIn }=\text { FALSE }) & 0 \\
\text { else } & -F_{I N R} \\
F_{\text {OUT }}=\text { if (boolForceIn = FALSE) } & F_{I N R} \\
\text { else } & 0
\end{array}
$$


Again, one manipulated variable or degree of freedom is lost by introducing this component, since one stream flow rate is zero necessarily.

\subsection{7- Model Sensitivity for a Consumer Plant}

Model sensitivity to parameters is shown and explained next for a diesel desulfurizer plant (G1). In the first experiment (Fig. 4.4), inputs regarding make-up and reactor parameters are changed, while in the second one (Fig. 4.5) those parameters corresponding to the solubility equilibrium in the separators are modified. Responses for the HP/MP/LP systems $\mathrm{H}_{2}$ purity and molecular weight (separators are named in the figures $s h p, s m p$, slp respectively), as well as important flow rates are shown. Average values (named $a v r g$ ) from historical laboratory data for intensive variables $\left(y^{H 2}, w^{L G}, w\right.$, named respectively $y, w i, w$ in the figures) are also represented. Three different sources are available for make-up, the two steam-reforming furnaces producer plants ( $\mathrm{H} 4$ and $\mathrm{H} 3$ ), and the high-pressure purge from a naphtha desulfurizer plant ( $\mathrm{N} 1$, whose sole make-up source is one of the catalytic-reforming plants). The G1 high-pressure purge goes to the LPH (named CBP in the figures).

\section{$\underline{\text { First experiment }}$}

Time 2, 3 Purity from N1 make-up is increased from 0.793 to $0.85 \% 1 \mathrm{~mol}$ and then decreased to the initial $0.793 \% 1 \mathrm{~mol}$. Compensated measurement $\left(d t F_{-} v c\right)$ is also represented for N1 make-up (FC_1), with the aim of pointing out the influence on the compensation.

Time 4, 5 Molecular weight from N1 make-up is increased a $15 \%$ from $24.3 \mathrm{~kg} / \mathrm{kmol}$ to $28.0 \mathrm{~kg} / \mathrm{kmol}$ and then decreased to the initial $24.3 \mathrm{~kg} / \mathrm{kmol}$. Compensated measurement $\left(d t F_{-} v c\right)$ is also represented for N1 make-up (FC_1), with the aim of pointing out the influence on the compensation.

Time $6 \mathrm{H}_{2}$ consumption in reactor is doubled from 30 to $60 \mathrm{Nm}^{3} \mathrm{H}_{2} / \mathrm{h}$ while maintaining the ratio $\Delta R_{L G} / \Delta R_{H 2}$, and make-up from producer plant $\mathrm{H} 4$ is increased from 0 to $5000 \mathrm{Nm}^{3} / \mathrm{h}$.

Time 8 In order to increase HP system $\mathrm{H}_{2}$ purity, make-up from producer plant $\mathrm{H} 4$ is increased from 5000 to $8000 \mathrm{Nm}^{3} / \mathrm{h}$, whereas make-up from N1 (from the catalytic-reforming producer plant P1) is reduced from 10700 to $8000 \mathrm{Nm}^{3} / \mathrm{h}$.

Time 10 Light ends generation in reactor is increased to a great extent, a 50\% from 1.5 to $2.25 \mathrm{Nm}^{3}$ LIG/h. 

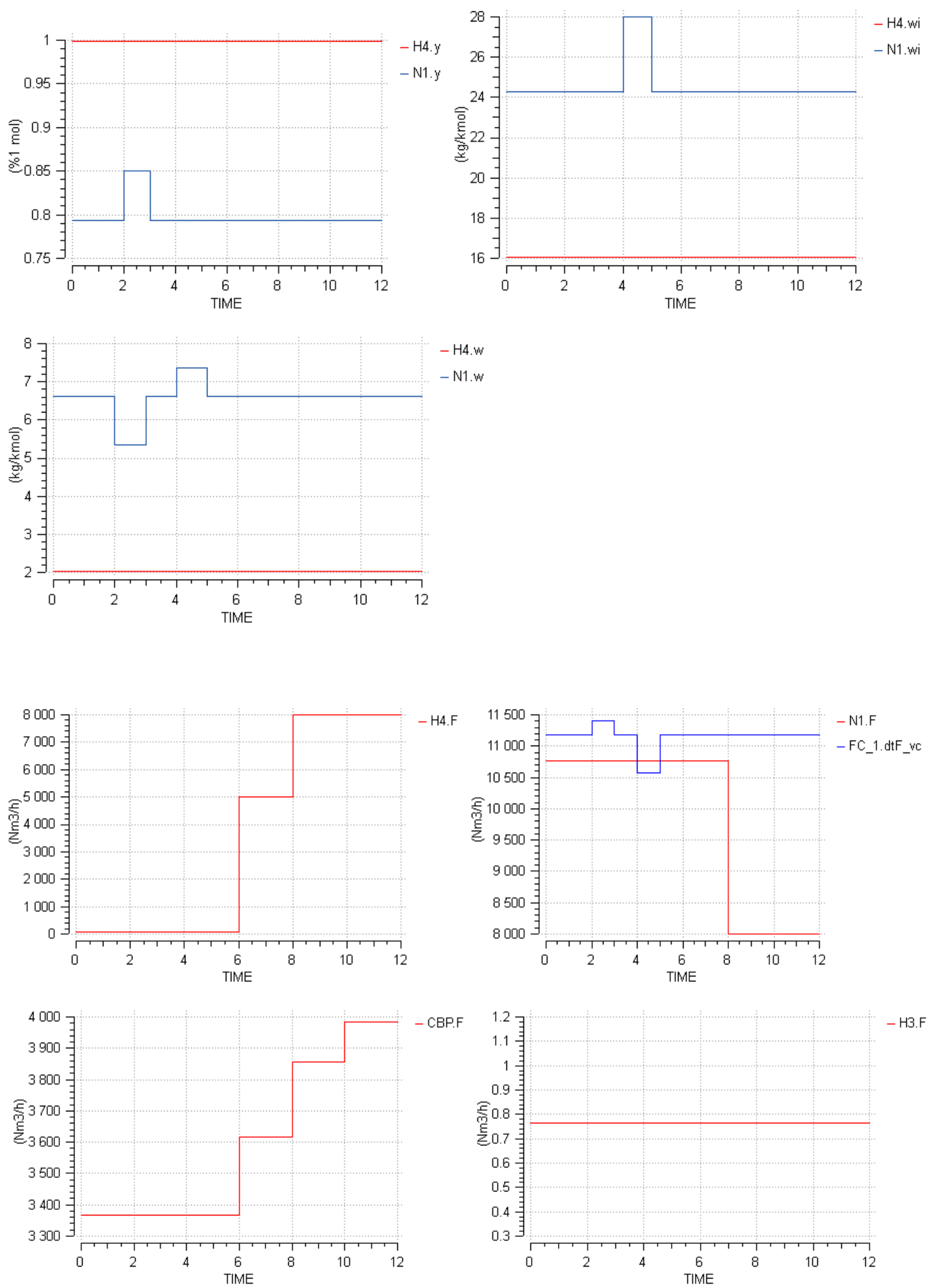

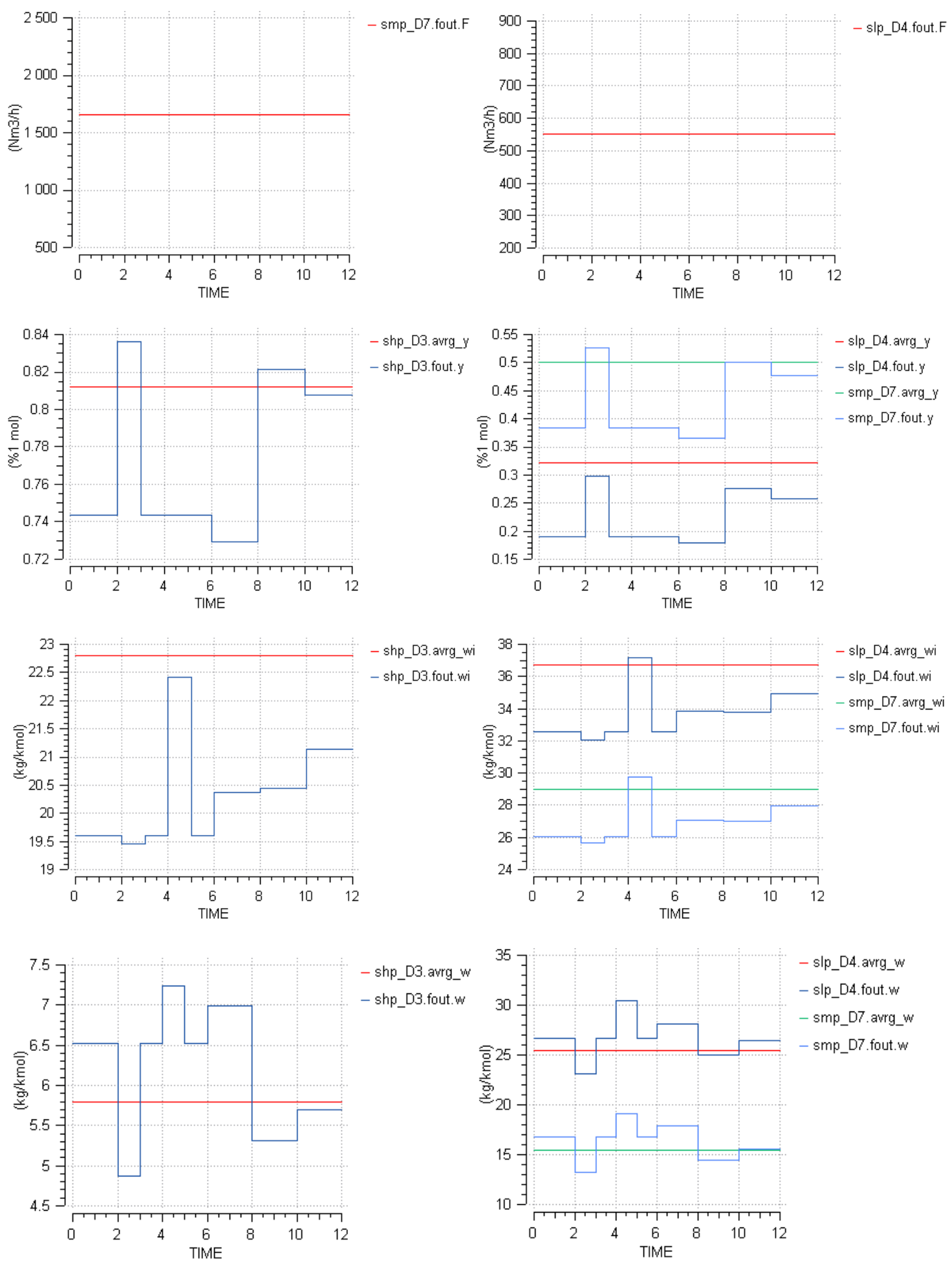

Figure 4.4. Model sensitivity to make-up and reactor parameters.

The HP system $\mathrm{H}_{2}$ purity $y_{H P}{ }^{H 2}$ is very sensitive to both flow rate $F$ and $y^{H 2}$ in make-up streams, as well as to consumption in reactor; whereas it is insensitive to variations in $w^{L / G}$ of make-up streams or light ends generated (the latter is not modified in the experiment). Both purities $y_{M P / L P}^{H 2}$ vary accordingly with $y_{H P}{ }^{H 2}$, and the same for $w_{M P / L P}{ }^{L / G}$ with $w_{H P}{ }^{L / G}$, when solubility equilibrium coefficients remain unchanged. It can be seen that $y_{H P}{ }^{H 2}$ increases with an increase 
in the make-up purity (Time $=2$ ), increases with an increase in a make-up flow rate of a higher purity (Time $=8$ ), decreases with an increase in the consumption in reactor $\Delta R_{H_{2}}$ (Time $=6$, partially counterbalanced with an increase in $\mathrm{H} 4$ make-up), and decreases with an increase in the light ends generation in reactor $\Delta R_{L I G}$ (Time=10), as expected. Regarding light ends generation in reactor (Time $=10$ ), the $y_{H P}{ }^{H 2}$ does not experience an important change even for a significant increase, due to the low ratio $\Delta R_{L I G} / \Delta R_{H 2}$ in normal operating conditions for all consumer plants.

Molecular weight $w$ for N1 make-up stream has a direct and significant influence over the N1 compensated flow rate ( $F C_{-} 1 . d t F_{-} v c$, being $F C_{-}$1.F=N1.F the model flow rate); the magnitude of the compensation depends also on the design value for the molecular weight.

The total purge to the LPH (CBP.F) is automatically established according to the mass balance for gas streams due to pressure control in the HP system, depending on the total make-up, gas solubility in the outlet liquid of the HP separator, and consumption and generation terms in reactor. Gas purged in the MP and LP separators do not vary if the corresponding solubility coefficient $k_{\text {gasHc }}^{\mathrm{S}}$ remains unchanged.

When the $y^{H 2}$ of a make-up stream increases, without any other change (Time=2), the $y_{H P}{ }^{H 2}$ increases with a high sensitivity; the corresponding variables for the MP and LP separators follow the same trend as for the HP ones, because being constant the solubility coefficients, both the outlet liquid and gas $y_{H P}{ }^{H 2}$ increase. However, the $w_{H P}{ }^{M G}$ experiences a negligible decrease; it is explained with the new equilibrium established by fulfilling material and solubility relations, although it can be comprehend thinking that with a smaller flow rate of light ends entering the system due to the higher $y^{\text {H2 }}$ for the $\mathrm{N} 1$ make-up stream, and the same $k_{\text {gasHC }}^{S}$ i.e. equal flow rate of gas solved in the liquid outlet of the separators, the removal of light ends increases in selectivity, and as a consequence a certain lighter light ends, thus with smaller $W_{H P}{ }^{L I G}$, are separated in both the gas and liquid streams of the HP separator.

When the $w^{L / G}$ of a make-up stream increases without any other change (Time=4), the $w_{H P}{ }^{L I G}$ increases with a high sensitivity; the $w_{M P / L P}{ }^{L / G}$ follow the trend for $w_{H P}{ }^{L / G}$, because of the constant solubility equilibrium coefficients. However, all $y^{\text {H2 }}$ for HP, MP and LP separators remain unchanged, because the equilibrium in purities is established irrespective of the light ends molecular weight and model mass balance equations. This is not completely accurate although reasonable enough according to process knowledge and operation, provided that the nature of light ends does not undergo significant changes from a mixture of methane, ethane, propane and in a smaller proportion other heavier gases, to a clear preponderance of methane without ethane and other heavier gases, as aforementioned, and also due to the narrow range in operating conditions regarding $y_{H P}{ }^{H 2}$. The implicit modelling assumption is that the gap in solubility for $\mathrm{H}_{2}$ with respect to the rest of light ends is big enough, and that lighter gases as methane that could compete with $\mathrm{H}_{2}$ will not be preponderant in the light ends composition, but keep between a certain range.

The increase in $w_{H P}{ }^{L / G}$ at Time $=6$ and Time $=10$ is related with an increase in the total flow rate of light ends generated in the reactor, because $w^{L / G}$ for generated $\Delta R_{L I G}$ is higher than the $w^{L / G}$ for the make-up gas. 


\section{Second experiment}

Time 2, 3 Solubility coefficient $k_{\text {gasHC }}^{S}$ is increased a 15\% in both HP/MP separators from $13.0 / 3.24 \mathrm{Nm}^{3} / \mathrm{m}^{3}$ to $14.9 / 3.73$, and then decreased to the initial $13.0 / 3.24$ $\mathrm{Nm}^{3} / \mathrm{m}^{3}$ respectively.

Time 5, 6 Solubility coefficient $k_{\alpha H 2 L I G}^{S}$ is increased a 25\% in both HP/MP separators from $5.75 / 2.63$ to $7.20 / 3.29$, and then decreased to the initial $5.75 / 2.63$ respectively.

Time 8, 9 Solubility coefficient $k_{\text {wLIG }}^{5}$ is increased a 15\% in both HP/MP separators from $0.70 / 0.80$ to $0.81 / 0.92$, and then decreased to the initial $0.70 / 0.80$ respectively.
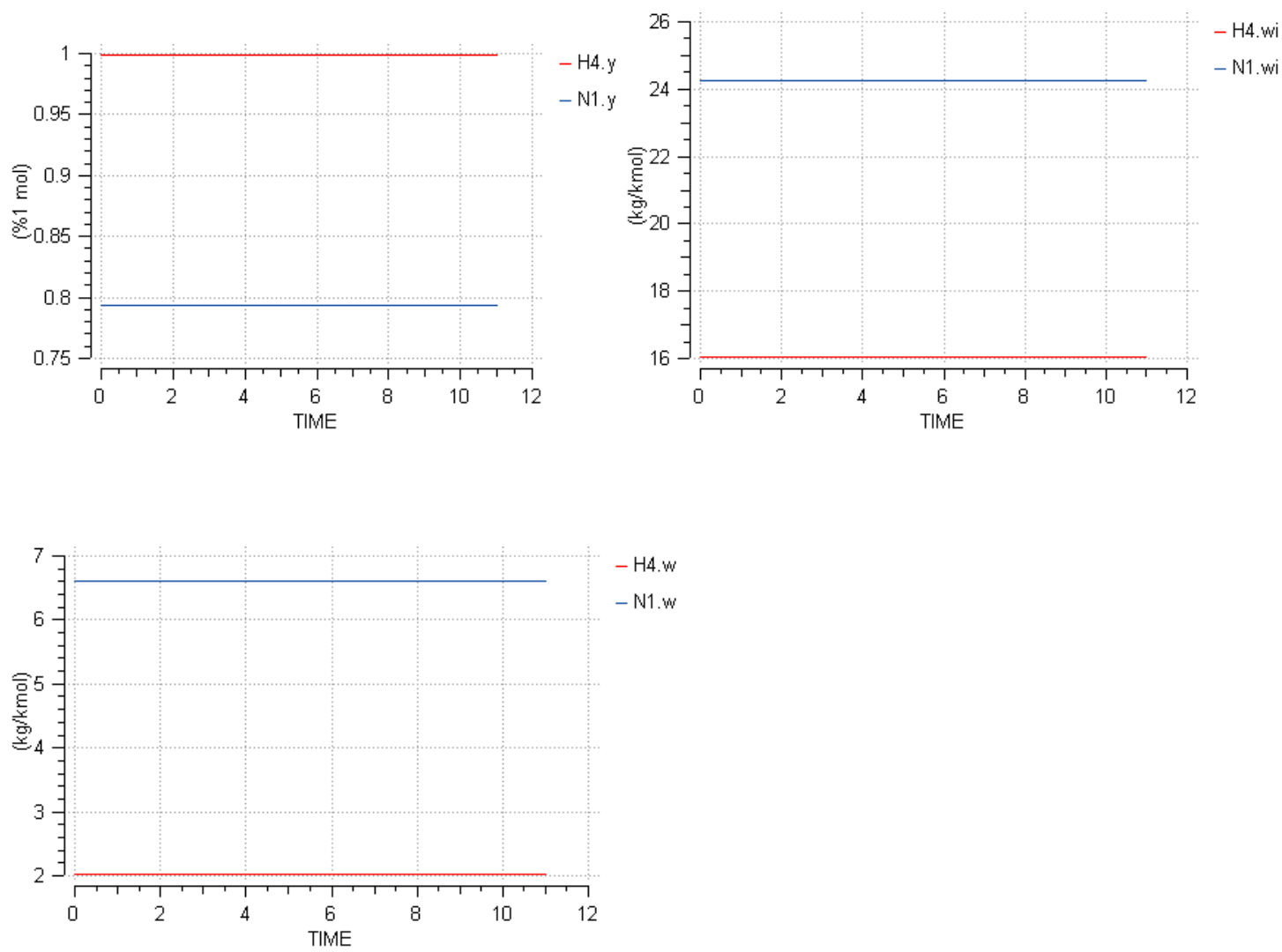

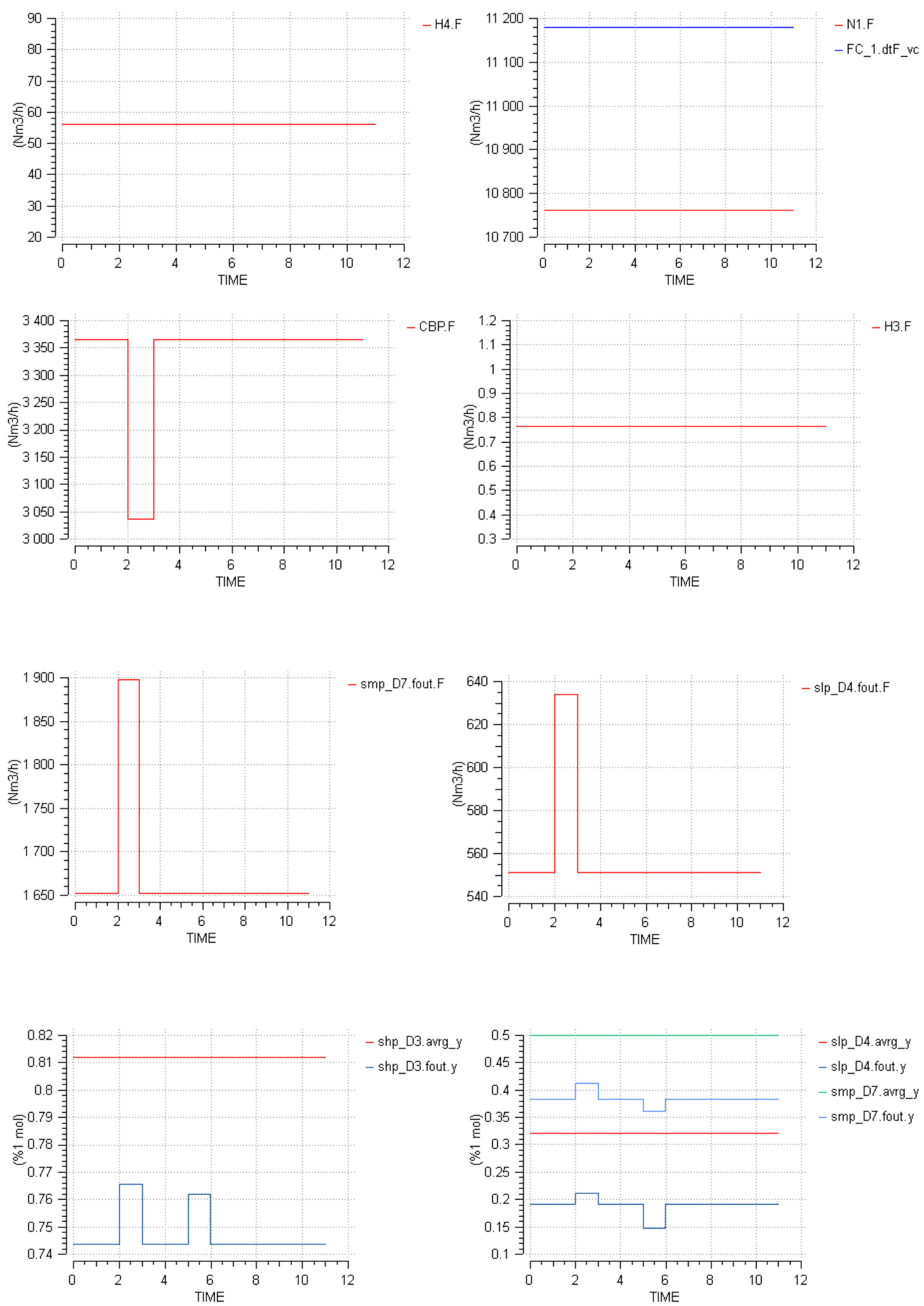

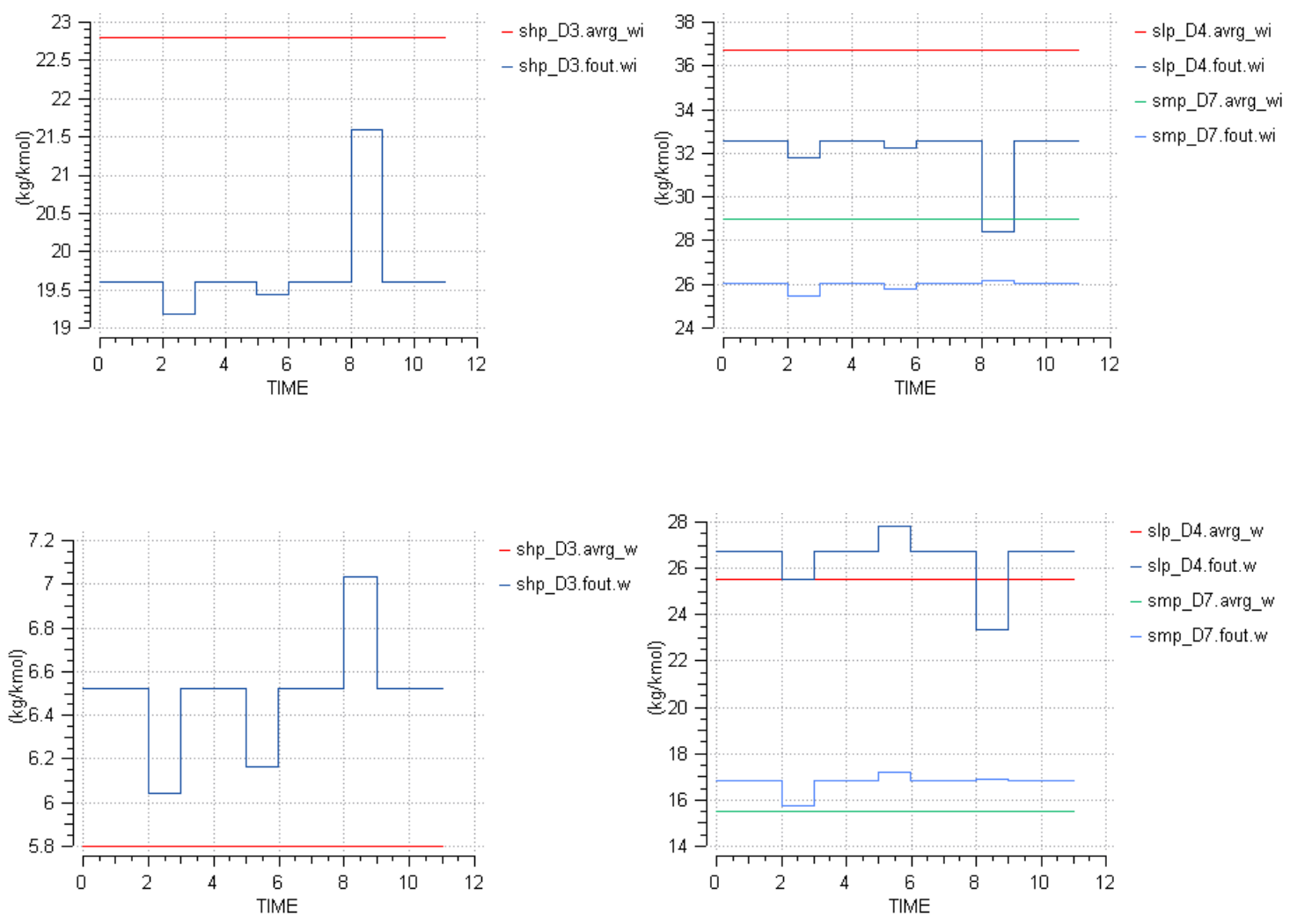

Figure 4.5. Model sensitivity to separators parameters.

When the gas solubility in hydrocarbon $k_{\text {gasHC }}^{S}$ increases at Time=2, the HP purge to the LPH (CBP.F) decreases consequently due to pressure control, while the total purge for both the MP and LP separators increases in the same amount. When the total gas solubility in liquid hydrocarbon increases, $\mathrm{H}_{2}$ purity $y^{\mathrm{H} 2}$ in all the HP, MP and LP separators increases because of a more selective separation, as light ends solve preferentially as compared to $\mathrm{H}_{2}$; for the same reason, $w^{L / G}$ in all the HP, MP and LP separators diminishes.

When the relative solubility of $\mathrm{H}_{2}$ with respect to light ends $k_{\alpha H 2 L G}^{S}$ is increased at Time $=5$, the $\mathrm{y}_{\mathrm{HP}}{ }^{\mathrm{H} 2}$ rises because of a more selective separation; this results in a drop in the $\mathrm{H}_{2}$ solved in the liquid outlet of the HP separator, therefore in a decline in the downstream $Y_{M P / L P}{ }^{H 2}$. For the same reason, $w_{H P}^{L / G}$ diminishes too, whereas $w_{M P / L P}^{L / G}$ is established according to the new equilibrium.

When the relative solubility of light ends $k_{\text {wLIG }}^{S}$ is increased at Time $=8$, all $y^{\mathrm{H} 2}$ for HP, MP and LP separators remain unchanged as aforementioned, because the equilibrium in purities is established irrespective of the light ends molecular weight and model mass balance equations. The $w_{H P}^{L / G}$ rises because of a less selective separation, or more equally distributed light ends between gas and liquid. For the $w_{M P}{ }^{L G}$, the smaller $w^{L G}$ at the inlet prevails over the effect of the higher $k^{S}{ }_{w L G}$ for the MP separator. The movement for the $w_{L P}{ }^{L I G}$ is difficult to ascertain, and depends on the three solubility coefficients for the HP and MP separators. 


\section{3- MODEL IMPLEMENTATION IN EcosimPro ${ }^{\circledR}$ ENVIRONMENT}

The hydrogen network model has been implemented in EcosimPro ${ }^{\circledR}$, which is a modelling and simulation environment based on the object-oriented paradigm, and supporting the following features:

- Graphical modeling and reusability of library components;

- Encapsulation, to hide complexity;

- Inheritance;

- Aggregation;

- Continuous and discrete modeling, with event handling;

The model for a real physical equipment is represented by means of an EcosimPro ${ }^{\circledR}$ component. Similarly, EcosimPro ${ }^{\circledR}$ ports serving as interface with the outside environment of a component are analogous to inlet/outlet material streams for physical equipment. A system can be modelled bottom-up with a modular development, where basic library components are combined and aggregated to create more complex components.

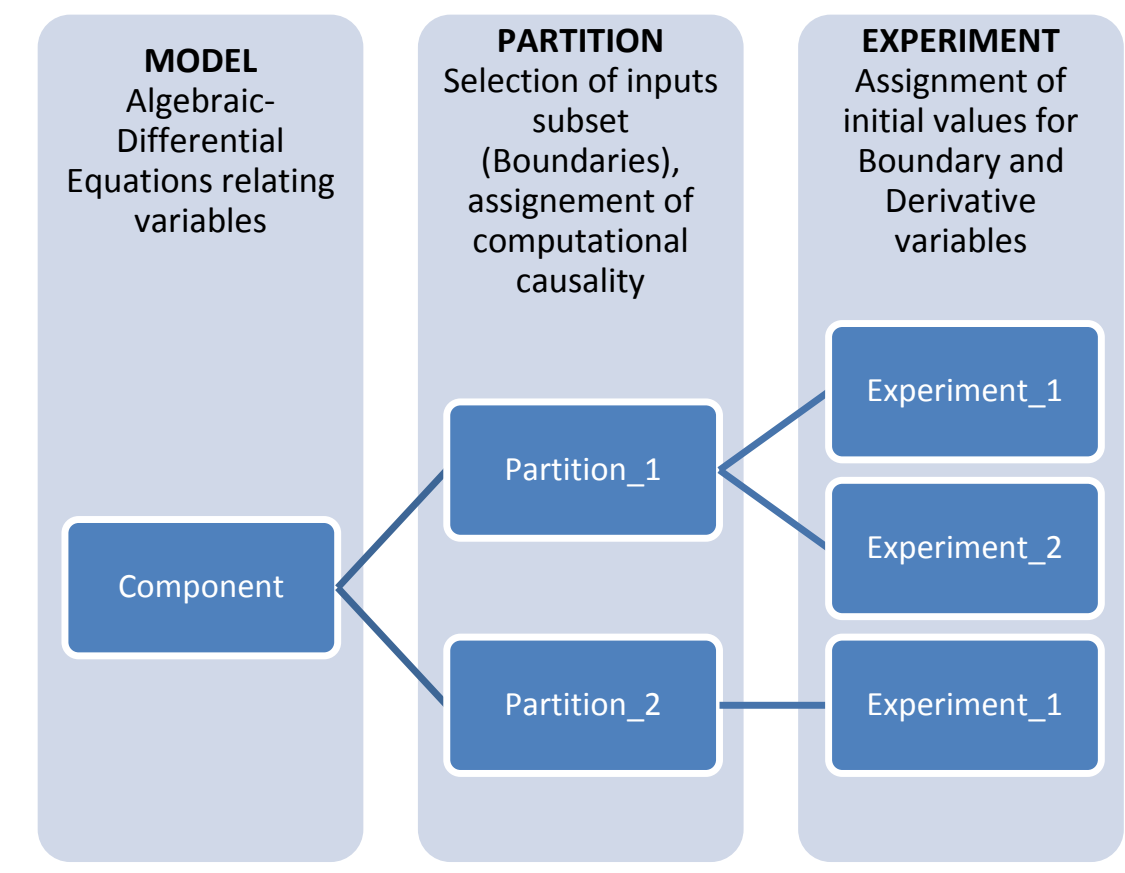

Figure 4.6. Modelling and computational structure of the EcosimPro ${ }^{\circledR}$ implementation.

EcosimPro ${ }^{\circledR}$ enables the sequential simulation of discrete-continuous systems. Modeling is organized hierarchically, according to "components", "partitions" and "experiments", whose function is described in Figure 4.6. Modelling equations are organized in components. The computation requires the specification of values for the independent variables or system degrees of freedom, called Boundaries. The selection of a subset of feasible variables to act as 
inputs or boundaries is made in the so called Partition, thus fixing the computational causality, according to which the remaining explicit and derivatives variables (both being outputs or dependent variables) are calculated from the system modelling equations. Particular values for boundary variables and initial values for derivative variables are assigned in the so called Experiment.

Developed components in the H2NET library will be shown next, and then the definition of the library ports will be explained, with certain particular characteristics. Then, the main ideas followed for the selection of the boundary set according to physical causality will be stated. Finally, additional features of the H2NET library will be described, useful for the automatic generation of the code to implement the optimizations necessary to solve the data reconciliation and optimal redistribution problems.

\subsection{1- EcosimPro ${ }^{\circledR}$ H2NET Model Library}

The following elementary components have been developed for the so called "H2NET" library, depicted in Figure 4.7; different library components account for different number of inlet/outlet ports. All the "H2NET" library components are briefly described in Table 4.1.

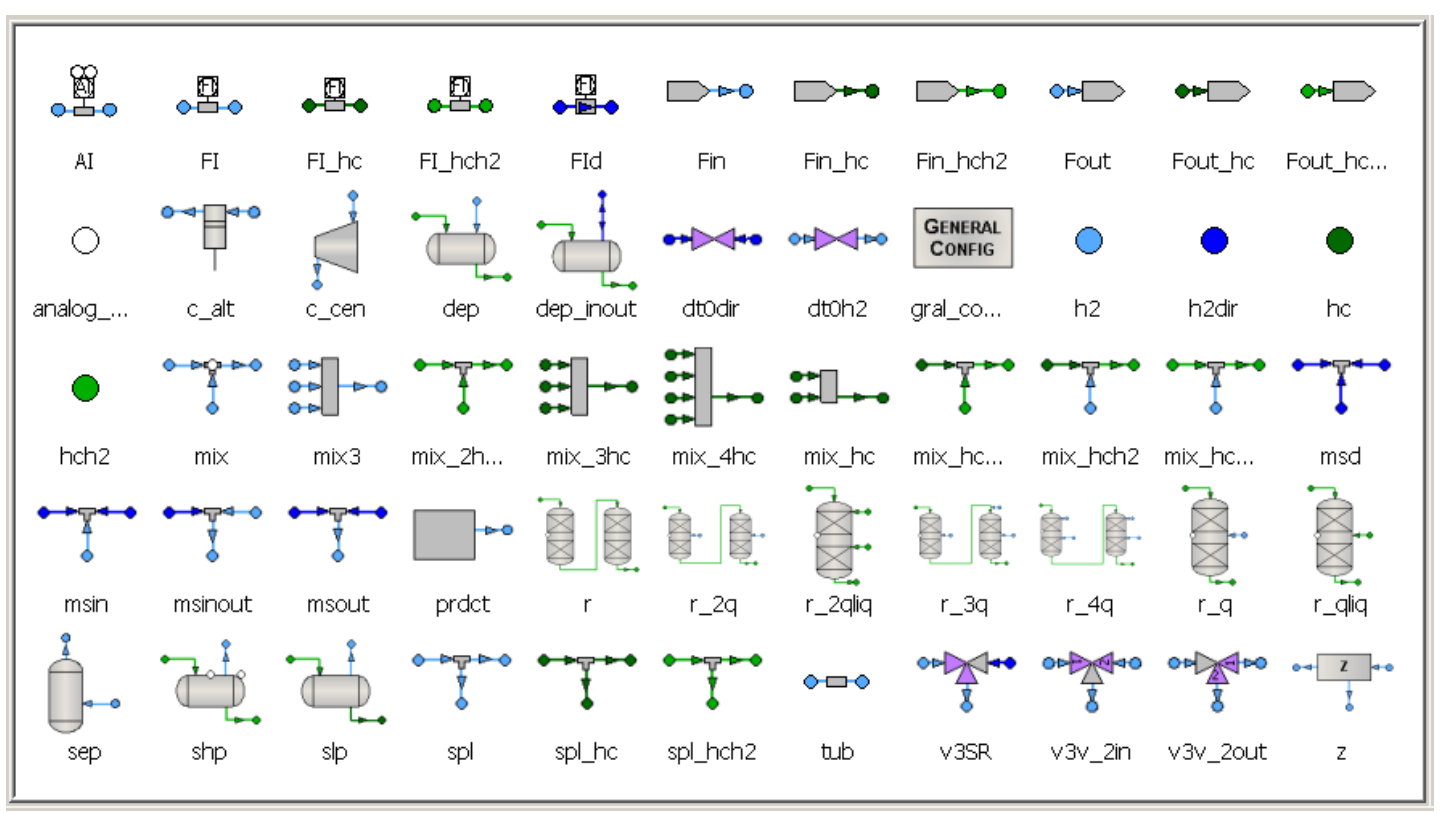

Figure 4.7. Components of the H2NET graphic library developed in EcosimPro ${ }^{\circledR}$. 


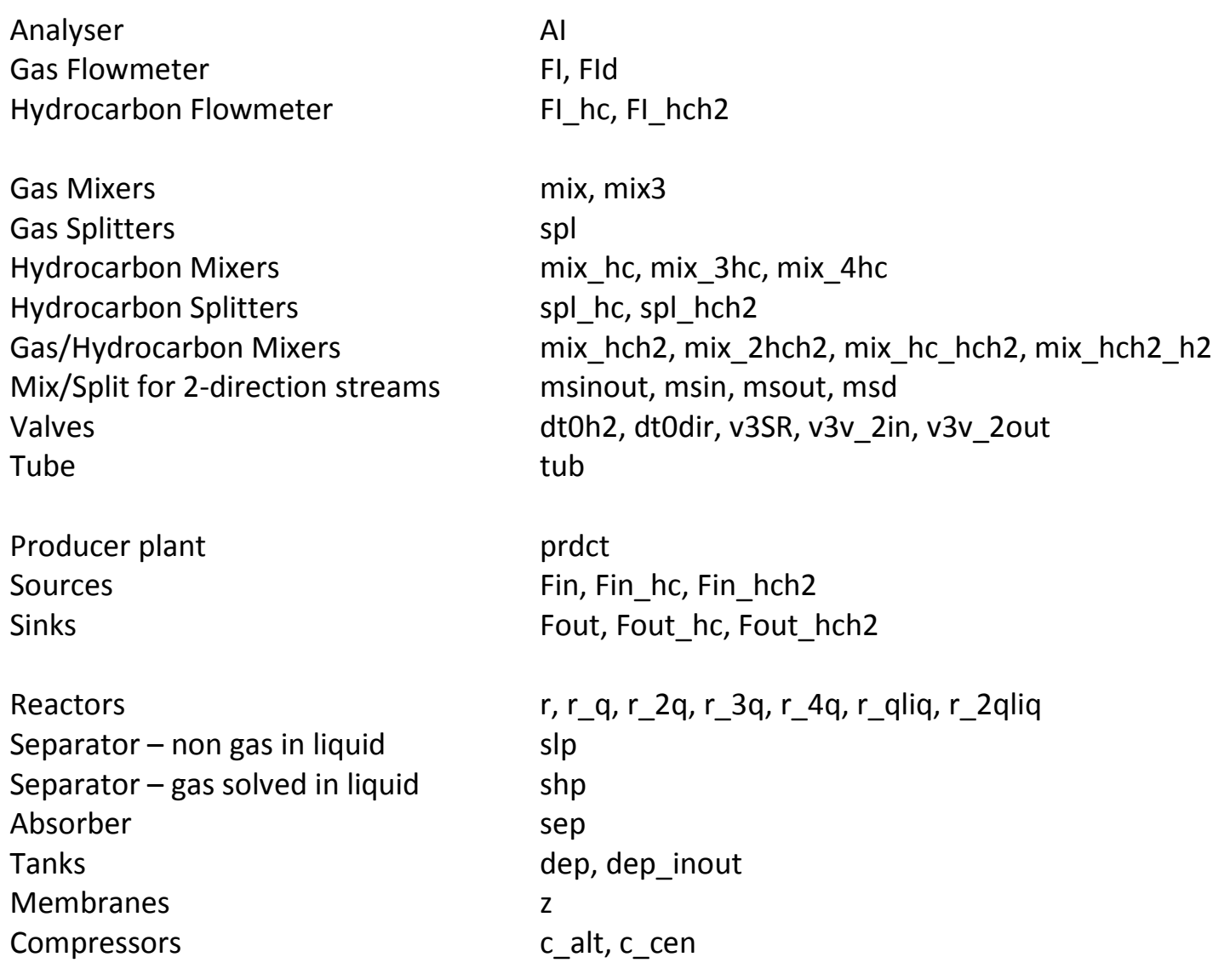

Table 4.1. Components of the H2NET library developed in EcosimPro ${ }^{\circledR}$.

The topology for the consumer and producer plants in the Petronor ${ }^{\circledR}$ refinery, constituting a higher level in the hierarchy, is built based on the "H2NET" library in the so called "H2NET_PETR" library. Some examples of consumer and producer plants flowsheet diagrams can be seen in Figures 4.8-4.10, whereas the global $\mathrm{H}_{2}$ network model, comprising all the consumer and producer plants as well as the connecting pipes, is shown in Figure 4.11. 


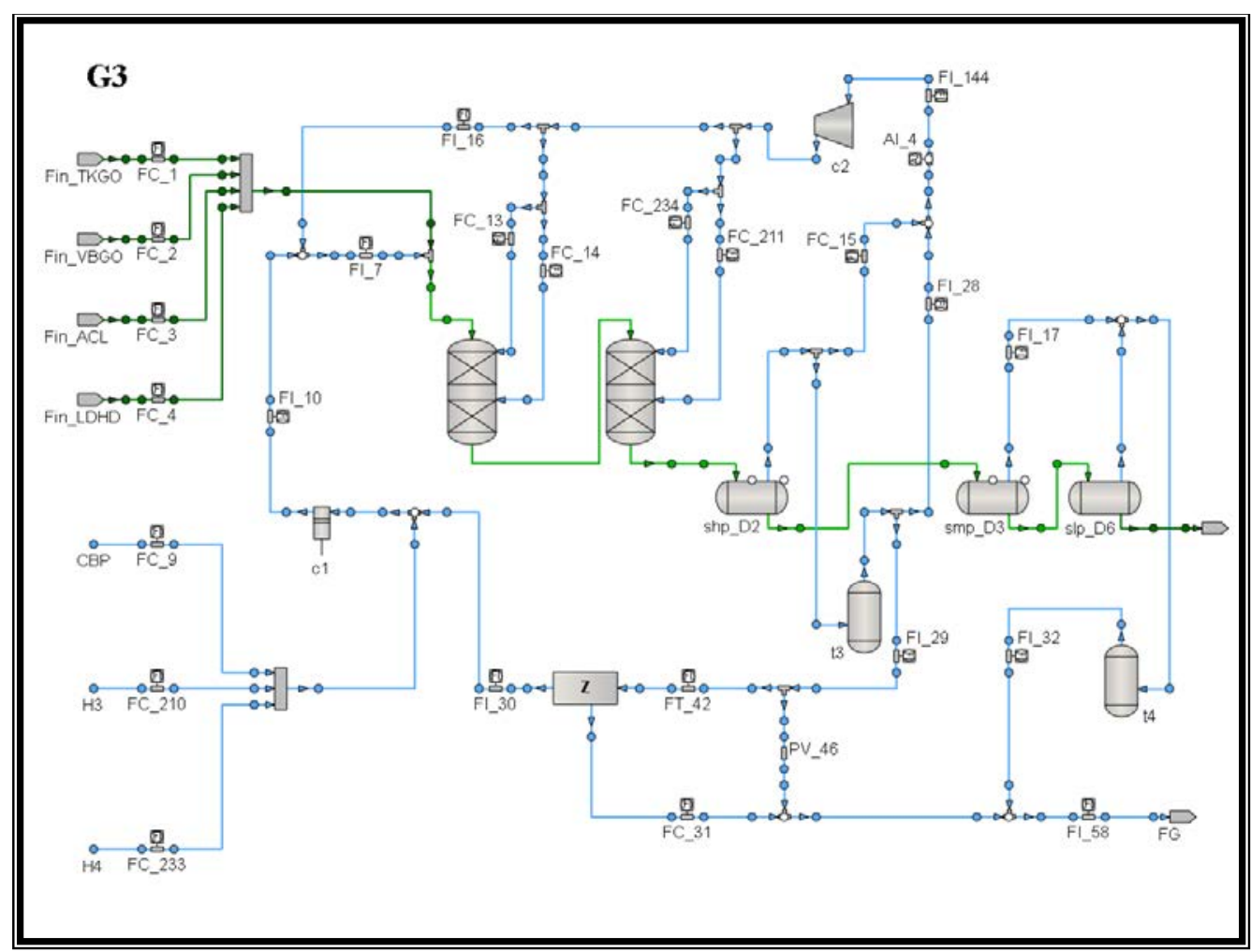

Figure 4.8. Flowsheet for a diesel hydrodesulfurizer consumer plant of the Petronor ${ }^{\circledR} \mathrm{H}_{2}$ network.

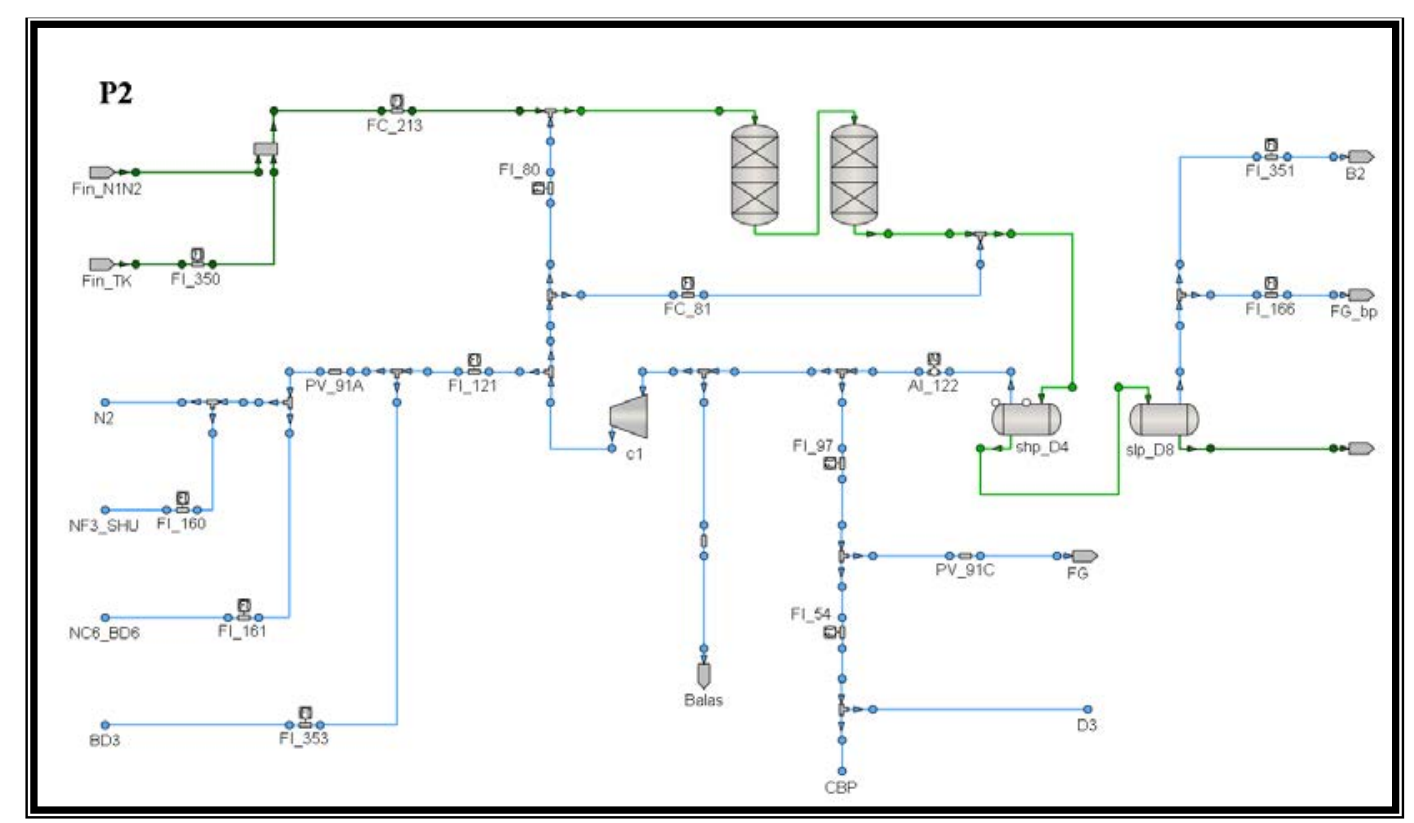

Figure 4.9. Flowsheet for a catalytic-reforming producer plant of the Petronor ${ }^{\circledR} \mathrm{H}_{2}$ network. 


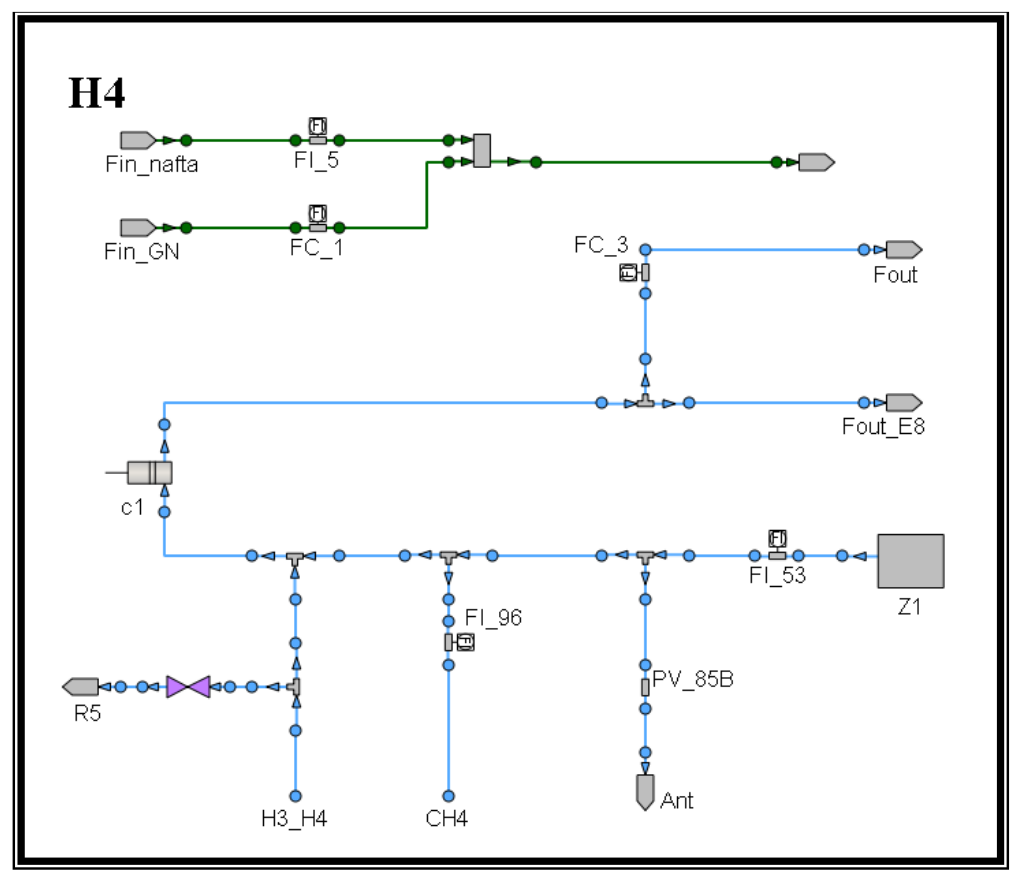

Figure 4.10. Flowsheet for a steam-reforming furnace producer plant of the Petronor ${ }^{\circledR} \mathrm{H}_{2}$ network.

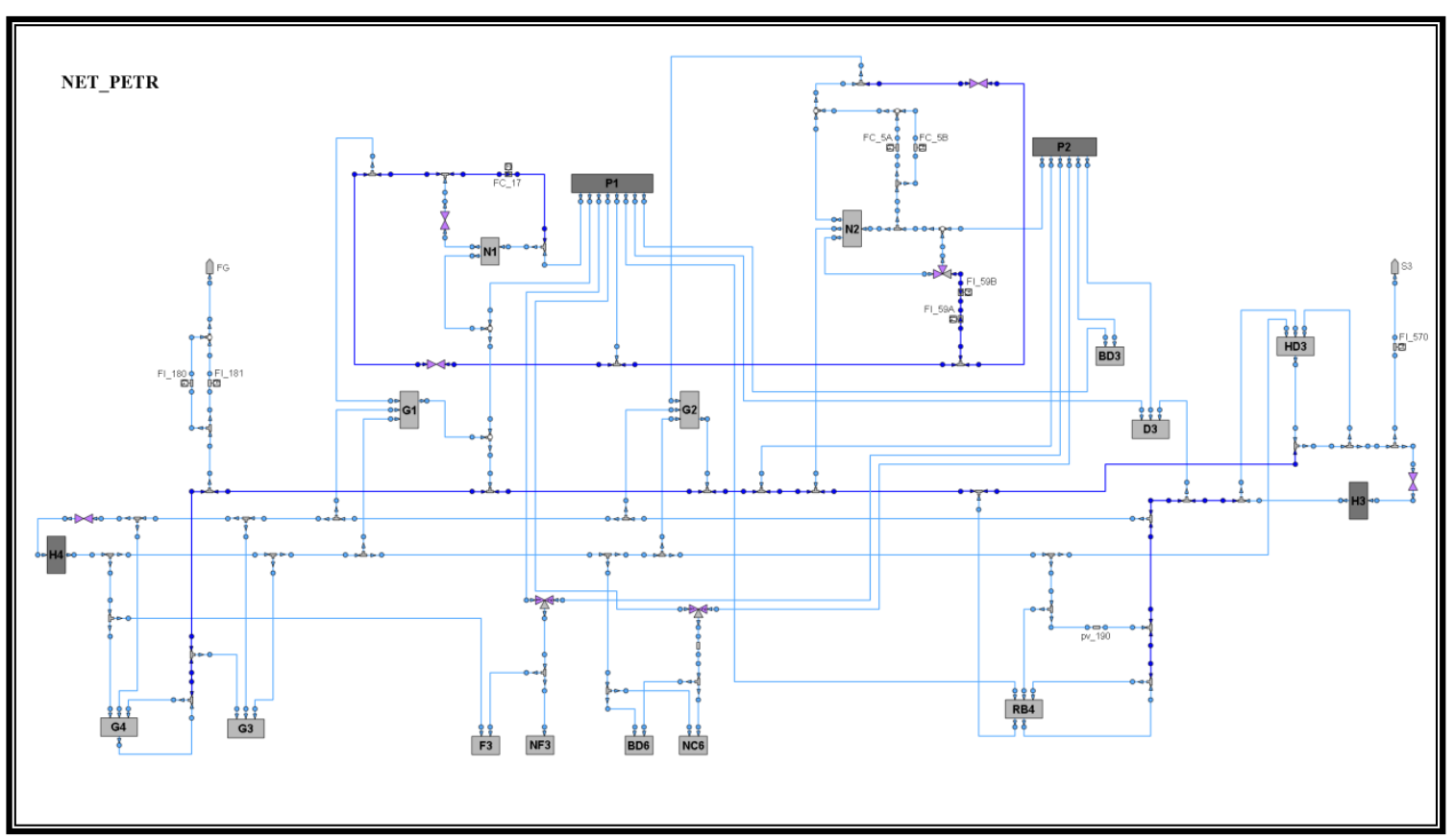

Figure 4.11. Diagram of the Petronor ${ }^{\circledR} \mathrm{H}_{2}$ network in EcosimPro ${ }^{\circledR}$, showing consumer and producer plants and available headers.

\subsection{2- Ports}

In the EcosimPro ${ }^{\circledR}$ environment, ports enable to connect components, both by communicating the values of the variables characterizing the port and by the equations that are applicable to the connections. In the modular modelling, components are connected using their ports; the 
connections create a composite component, which may in turn have ports which are connected internally to ports of the components which have been instantiated. Models for consumer and producer plants are composite components created from elements of the H2NET library, which can be connected using their external ports to model the site network; components for consumer and producer plants constitute a higher level in the hierarchy.

Different ports are defined for the different material streams; they are described in Table 4.2, with the associated colour. Model representation for mixed hydrocarbon-gas streams does not distinguish whether there is one phase (gas solved in liquid hydrocarbon) or two phases (gas solved in liquid hydrocarbon coexisting with gas phase). In the model for "hch2" streams, hydrocarbon and gas has been considered separately, and variables characterizing the port are analogous to those for hydrocarbon liquid streams together with those for gas streams.

\begin{tabular}{lll}
\hline Material Stream & H2NET Library Ports \\
\hline Gas with known direction & h2 \\
Gas with 2 allowed directions & h2dir \\
Hydrocarbon with solved gas & hch2 \\
Liquid Hydrocarbon without solved gas & hc \\
\hline
\end{tabular}

Table 4.2. Ports defined in the H2NET library.

Only ports of the same type can be connected among them. When used in components, a direction must be specified for each port used, either IN or OUT, meaning that it is either an inlet or an outlet stream.

EcosimPro ${ }^{\circledR}$ language makes available optional modifiers for the declared variables, which enable the automatic generation of equations for port connections. None automatic equation is generated in port connections for variables not affected by any modifier.

The SUM modifier for a declared variable (typically extensive ones) implies that an equation to equal the sum of variables for IN streams with the sum for OUT streams for connected ports is generated, when multiple connections are allowed not only single ones. The EQUAL modifier for a declared variable (typically intensive ones) implies that an equation to equal the variable for all connected ports is generated. The aforementioned modifiers SUM and EQUAL can be further delimited with the modifiers IN or OUT, which mean that the equation is only generated when the multiple connection has the direction specified. When all connections are single ones, as is the case, there is no difference among a declaration as SUM or SUM IN or 
SUM OUT, and the same occurs for the EQUAL modifier; furthermore, the equation generated is the same for both SUM and EQUAL modifiers.

\section{“h2" port}

The port for gas streams is named " $h 2$ ", and the variables declared are specified in Table 4.3. The definition of the " $h 2$ " port prevents mistakes while connecting components, for example connecting two inlet ports between them or two outlet ports. This is thanks to the modifiers IN and OUT added to SUM and EQUAL for certain port variables.

\begin{tabular}{|c|c|c|c|}
\hline Variable & Description & Units & Modifier \\
\hline$F$ & Volumetric flow rate at Normal Conditions & $\mathrm{Nm}^{3} / \mathrm{h}$ & SUM IN \\
\hline$y$ & Hydrogen purity & $\% 1 \mathrm{~mol}$ & EQUAL OUT \\
\hline$F_{H 2}$ & $\mathrm{H}_{2}$ volumetric flow rate at Normal Conditions & $\mathrm{Nm}^{3} \mathrm{H}_{2} / \mathrm{h}$ & \\
\hline$W$ & Mass flow rate & $\mathrm{kg} / \mathrm{h}$ & \\
\hline$w$ & Gas stream molecular weight & $\mathrm{kg} / \mathrm{kmol}$ & \\
\hline$w^{L L G}$ & Light ends/Impurities molecular weight & $\mathrm{kg} / \mathrm{kmol}$ & EQUAL OUT \\
\hline$W_{\mathrm{H} 2}$ & $\mathrm{H}_{2}$ mass flow rate & $\mathrm{kg} \mathrm{H}_{2} / \mathrm{h}$ & \\
\hline$\rho$ & Density at Normal Conditions & $\mathrm{kg} / \mathrm{Nm}^{3}$ & \\
\hline$T$ & Temperature & K & EQUAL OUT \\
\hline
\end{tabular}

Table 4.3. Port " $h 2$ ": variables and description.

As aforementioned, the complete characterization of a gas stream $i$ is performed with the three independent variables: gas flow rate at Normal conditions, equivalent to molar flow rate $F_{i}\left(\mathrm{Nm}^{3} / \mathrm{h}\right), \mathrm{H}_{2}$ purity $y_{i}^{\mathrm{H} 2}(\% 1 \mathrm{~mol})$ and molecular weight for light ends $w_{i}^{\mathrm{LIG}}(\mathrm{kg} / \mathrm{kmol})$, and equations to automatically transfer these three variables through any connection between two ports are generated with the modifiers. The equations modelling the port relations among variables are stated next, corresponding to each port. Certain constants are included, as the equivalence for ideal gases in normal conditions $22.415 \mathrm{Nm}^{3} / \mathrm{kmol}$, and $\mathrm{H}_{2}$ molecular weight $w^{\text {H2 }}=2.01588 \mathrm{~kg} / \mathrm{kmol}$.

$$
\begin{aligned}
& F_{H 2}=F \cdot y \\
& W=(F / 22.415) \cdot w \\
& W_{H 2}=\left(F_{H 2} / 22.415\right) \cdot w^{H 2} \\
& w=w^{H 2} \cdot y+w^{L I G} \cdot(1-y) \\
& \rho=w / 22.415
\end{aligned}
$$




\section{"h2dir" port}

The port " $h 2 d i r$ " comprises all the variables and behavior of " $h 2$ " port, with some additional ones. Whereas the " $h 2$ " port is intended for streams with a unique direction of flow, the purpose of the "h2dir" port is to model streams whose direction is not known in advance, or could be both the direct and reverse ones. In the $\mathrm{H}_{2}$ network this can occur in connecting pipes and headers, but not in equipment as reactors and separators and in general inside the plants.

According to the physical causality, which coincides with the computational causality with the selection of boundaries made, all qualitative or intensive variables for an inlet stream to a certain component mixer or splitter are known upstream, and only those qualitative or intensive variables for the outlet streams are computed following the model and behaviour for the said component. The difficulty arises in components such as mixers and splitters, because due to the unknown direction of flow for one or several streams, these mixers and splitters can not be classified as such: the computational causality is not determined until the direction of flows, that is, the total material balance, is solved, and then variables regarding composition can be computed accordingly. However, the number of equations for each EcosimPro ${ }^{\circledR}$ component and port has to be specified in advance and irrespective of the physical causality regarding flows, which can be subject to changes during simulation.

As a consequence, since one additional equation for each intensive variable is needed in the splitter component as compared to the mixer one (abovementioned eq. 43 added to determine the composition $y_{\text {OUT }}{ }^{H 2}$ and $w_{\text {OUT }}{ }^{4 G}$ for one of the outlet streams), the solution adopted is to model the "h2dir" ports with one equivalent variable for each intensive one unpaired, without equation to be computed in the port. The unpaired variable will be used to make the corresponding assignment for composition in components whenever needed to accomplish symmetry in modelling equations: as it is not known in advance whether a component will behave as a mixer or as a splitter, both behaviours are modelled simultaneously, and the unpaired port variable enables to complete the branch corresponding to an inlet stream whose composition should have been computed upstream, thus not needing a modelling equation in the component and employing for symmetry this unpaired port variable. This implementation enables to spread the values of the intensive variables through the whole subsystem of "h2dir" ports connected among them, wherever the upstream-todownstream sequence starts. The auxiliary unpaired variable is just an intermediate, enabling to assign an even and symmetrical number of conditions irrespective of flow direction, because direction for flow is not known at the scope of a single component, but at the scope of the global network.

For every component with a " $h 2 d i r$ " port, a construction parameter is defined linked to each "h2dir" port, named "modefdir". The construction parameter "modefdir" has two allowed values "fdin" and "fdout", which are configured in modeling time before model compilation, and is used to assure that the equations for intensive variables computation are generated only once per connection, not twice; therefore it is mandatory when connecting two " $h 2 d i r$ " ports that the linked parameters corresponding to the two components are set to " $f d i n$ " and "fdout" or viceversa, not being permitted two "fdin" or two "fdout". A message for the user is 
automatically generated in model compilation to prevent mistakes while configuring " $h 2 d i r$ " ports, whenever two "fdin" or two "fdout" are connected between them.

The " $h 2 d i r$ " port has been defined as inlet port for every component, thus the port flow rate $F$ has positive sign when it is truly an inlet regarding the component, and negative sign when it is an outlet. Connections IN-IN are allowed between components for "h2dir" ports, as no variable has been declared as SUM IN or EQUAL OUT. Variables declared in the port are described in Table 4.4, and the additional equations with respect to the " $h 2$ " port are also included, thus remaining as unpaired the intensive variables $y_{\text {OUT, }}, w_{\text {OUT }}, T_{\text {OUT }}$.

\begin{tabular}{|c|c|c|c|}
\hline Variable & Description & Units & Modifier \\
\hline$F$ & Volumetric flow rate at Normal Conditions & $\mathrm{Nm}^{3} / \mathrm{h}$ & SUM \\
\hline$y$ & Hydrogen purity & $\% 1 \mathrm{~mol}$ & \\
\hline$F_{H 2}$ & $\mathrm{H}_{2}$ volumetric flow rate at Normal Conditions & $\mathrm{Nm}^{3} \mathrm{H}_{2} / \mathrm{h}$ & \\
\hline$W$ & Mass flow rate & $\mathrm{kg} / \mathrm{h}$ & \\
\hline$w$ & Gas stream molecular weight & $\mathrm{kg} / \mathrm{kmol}$ & \\
\hline$w^{L / G}$ & Light ends/Impurities molecular weight & $\mathrm{kg} / \mathrm{kmol}$ & \\
\hline$W_{\mathrm{H} 2}$ & $\mathrm{H}_{2}$ mass flow rate & $\mathrm{kg} \mathrm{H}_{2} / \mathrm{h}$ & \\
\hline$\rho$ & Density at Normal Conditions & $\mathrm{kg} / \mathrm{Nm}^{3}$ & \\
\hline$T$ & Temperature & $\mathrm{K}$ & \\
\hline$y_{I N}$ & Hydrogen purity & $\% 1 \mathrm{~mol}$ & EQUAL \\
\hline YOUT & Hydrogen purity & $\% 1 \mathrm{~mol}$ & EQUAL \\
\hline$w_{I N}$ & Gas stream molecular weight & $\mathrm{kg} / \mathrm{kmol}$ & EQUAL \\
\hline$w_{\text {OUT }}$ & Gas stream molecular weight & $\mathrm{kg} / \mathrm{kmol}$ & EQUAL \\
\hline$T_{\text {IN }}$ & Temperature & $\mathrm{K}$ & EQUAL \\
\hline$T_{\text {OUT }}$ & Temperature & K & EQUAL \\
\hline
\end{tabular}

Table 4.4. Port "h2dir": variables and description.

$$
\begin{gathered}
y=y_{I N} \\
w=w_{I N} \\
T=T_{I N}
\end{gathered}
$$

It is illustrated in Figure 4.12, where the two simplest components with "h2dir" ports are

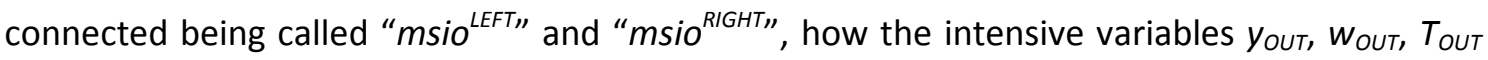
are used to guarantee an even number of conditions in branches while at the same time allowing for causality, which causes a lack of symmetry and an odd number of constraint equations on the related components depending on the flow direction. Parameter " $f d$ " has

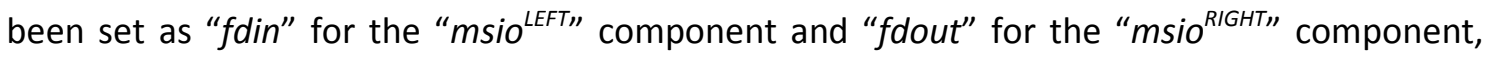
although it is interchangeably. 


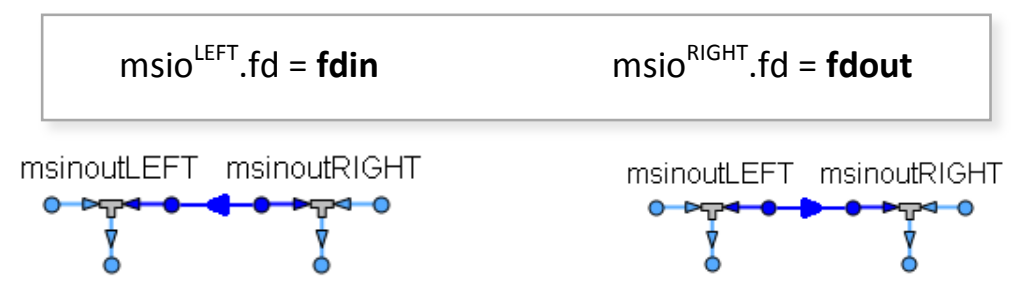

\begin{tabular}{|c|c|}
\hline 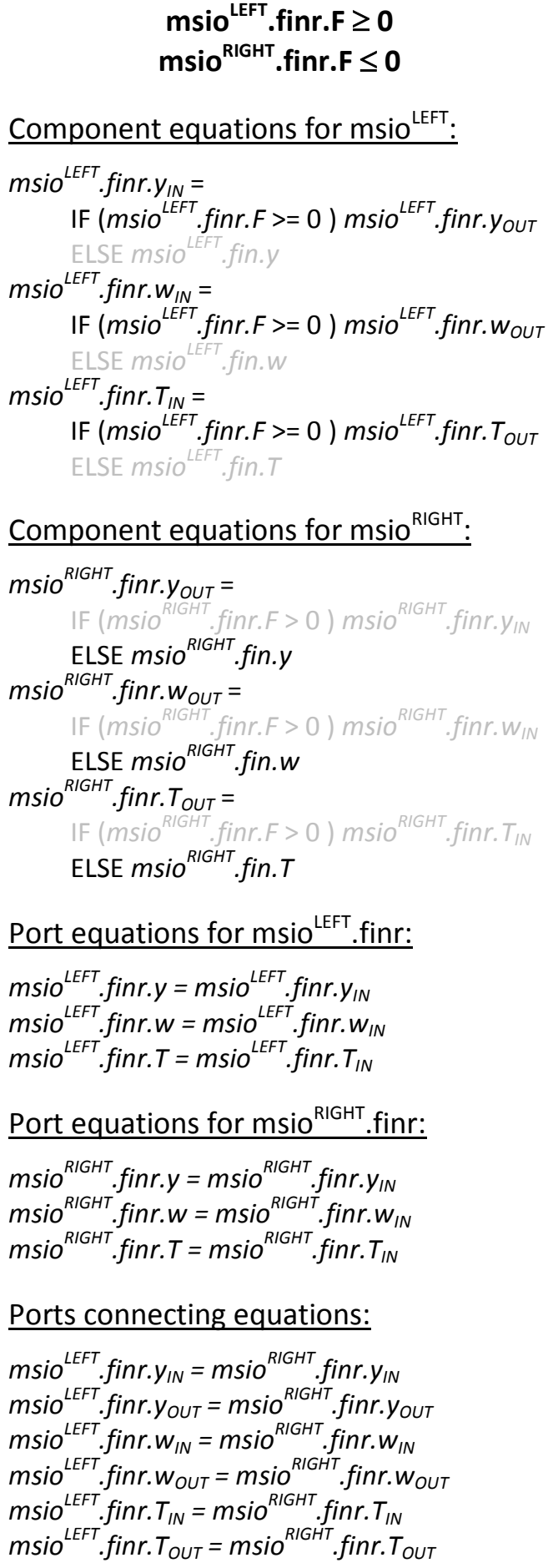 & 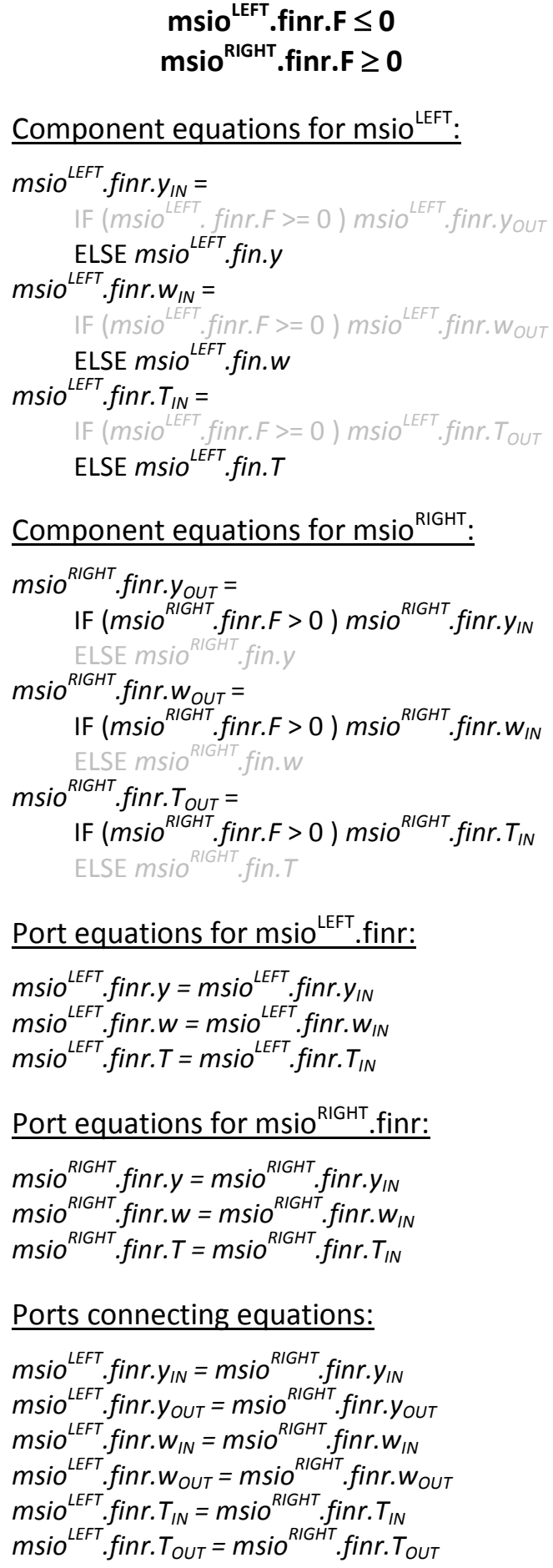 \\
\hline
\end{tabular}

Figure 4.12. Example of intensive variable computation for flow direction change. 
The component "msinout" has three ports, namely "fin", "fout" and "finr". According to physical causality, intensive variables for "fin" port must be either boundary conditions or either computed in an upstream component, therefore already known. Intensive variables for "fout" port are calculated from the component modelling equations, that is, the corresponding material balances. The sequence of computations for the "finr" port intensive variables is stated below depending on the "finr" flow direction, for the two possible flow directions. The branch not active in each case is shown in grey.

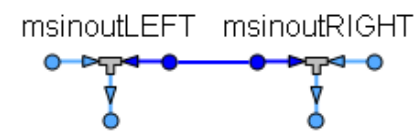

The system of equations enables to univocally determine all the ports and components variables. The value of the "modefdir" parameter determines which variable, whether intensive $_{\text {IN }}$ or intensive OUT, $_{\text {, }}$ is explicitly calculated with the component equations. The conditions for all the branches depend on the flow direction for those streams with direction unknown in advance. When the component "msinout" behaves as a splitter, being the "finr" port flow rate $F$ negative (outlet stream), then the intensive variable intensive IN/OUT $_{\text {is }}$ equal to the corresponding intensive for the component inlet stream "fin". Nevertheless, when the component behaves as a mixer, being the "finr" port flow rate $F$ positive (inlet stream), then the intensive variable intensive IN/OUT $_{\text {is }}$ made equal to the port variable without pairing, that is

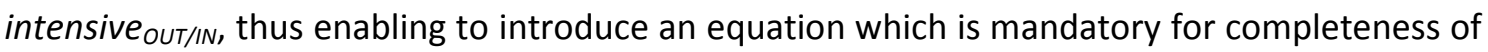
all the branch conditions while at the same time not forcing a value for this intensive variable in the 'mixer' component, because due to physical causality it must have been computed upstream, and just letting the information to be transferred through the port connecting equations.

It is worthy to mention that a discontinuity arises for intensive variables $\left(y^{H 2}, w^{L / G}\right)$ when a "h2dir" stream changes the flow direction: there is a switch from the operating conditions in one extreme to the ones in the other extreme, due to physical causality. Nevertheless, these discontinuities have not proven detrimental for the resolution of the subsequent optimization problems, in spite of the discontinuity both in the variables and in the derivatives. In fact, the flow rate is a continuous function passing through zero, thus all terms in equations for balances are continuous because purities and molecular weights are multiplied by flow rates; moreover, these intensive variables do not experience big changes in normal operating conditions, being discontinuities of a small magnitude. An experiment was made where all discontinuities were removed, and the bulk outlet composition was approximately estimated by a function of all inlets to the headers without considering changes in composition in different zones of the headers, and the optimization results did not improve neither in execution time nor in accuracy as compared to those considering the actual model with the discontinuities.

The same approach can be extended in a straightforward way for similar components with two or three gas streams with unknown direction. In these cases, conditions for the branches 
depend on all the combination of allowed directions for streams. A brief extract of the modelling code for components "msin", "msout" and "msd" is included below, with two or three streams with two allowed directions for flow respectively.

The model for the component "msinout" is also shown. The three material balances enable to determine and completely characterize the outlet stream "fout", by computing the variables fout. $F$, fout.y, fout.w; whereas the conditional equations generated in compilation time as a function of the particular configuration for the construction parameter " $f d$ ", being " $f d$ " of the declared type "modefdir" with allowed values "fdin" and "fdout", are aimed at transmitting the intensive variables for composition according to physical causality following the flow direction.

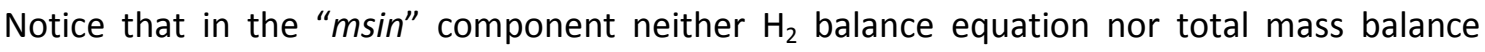
equation are needed because no outlet port is declared. 


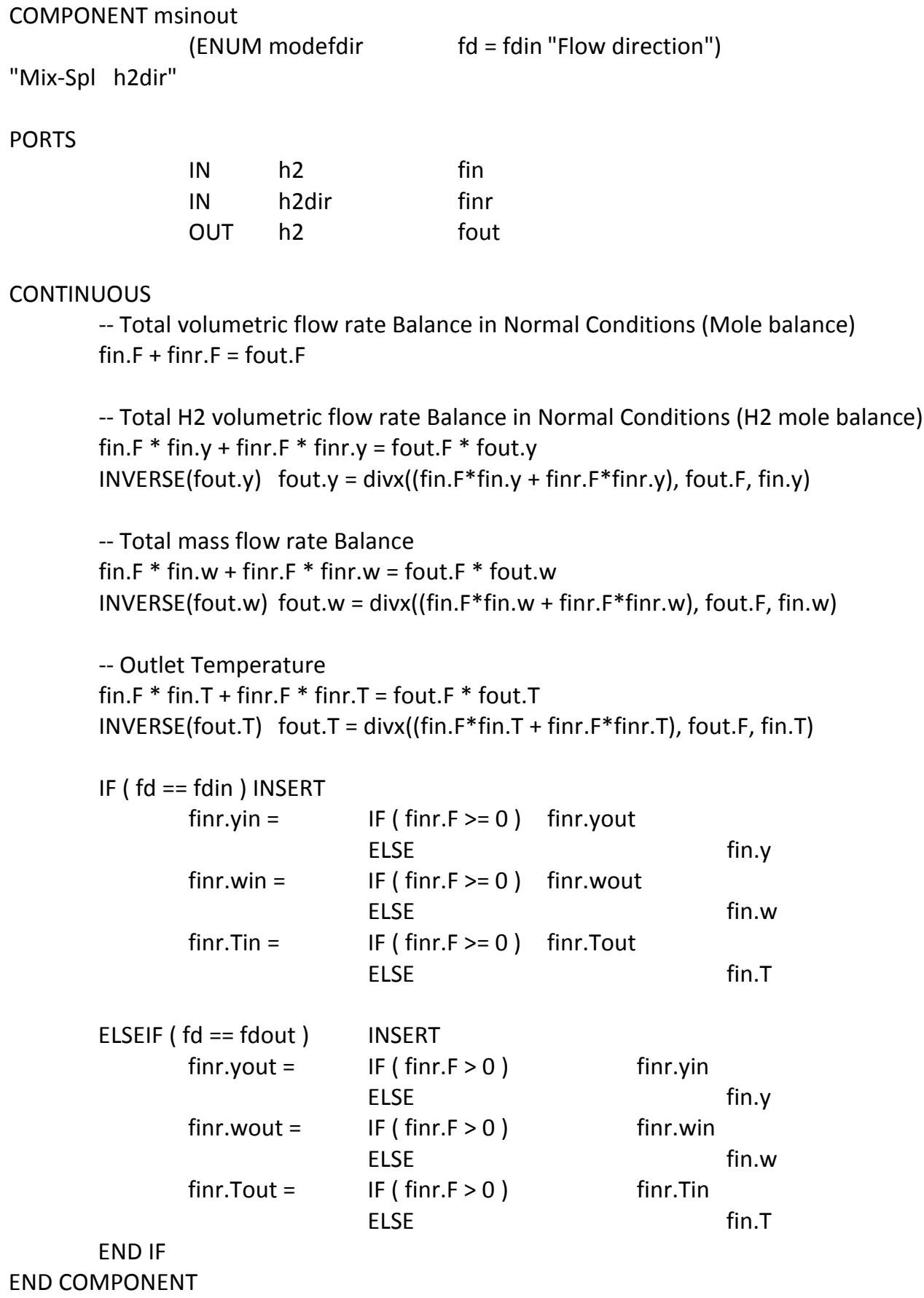




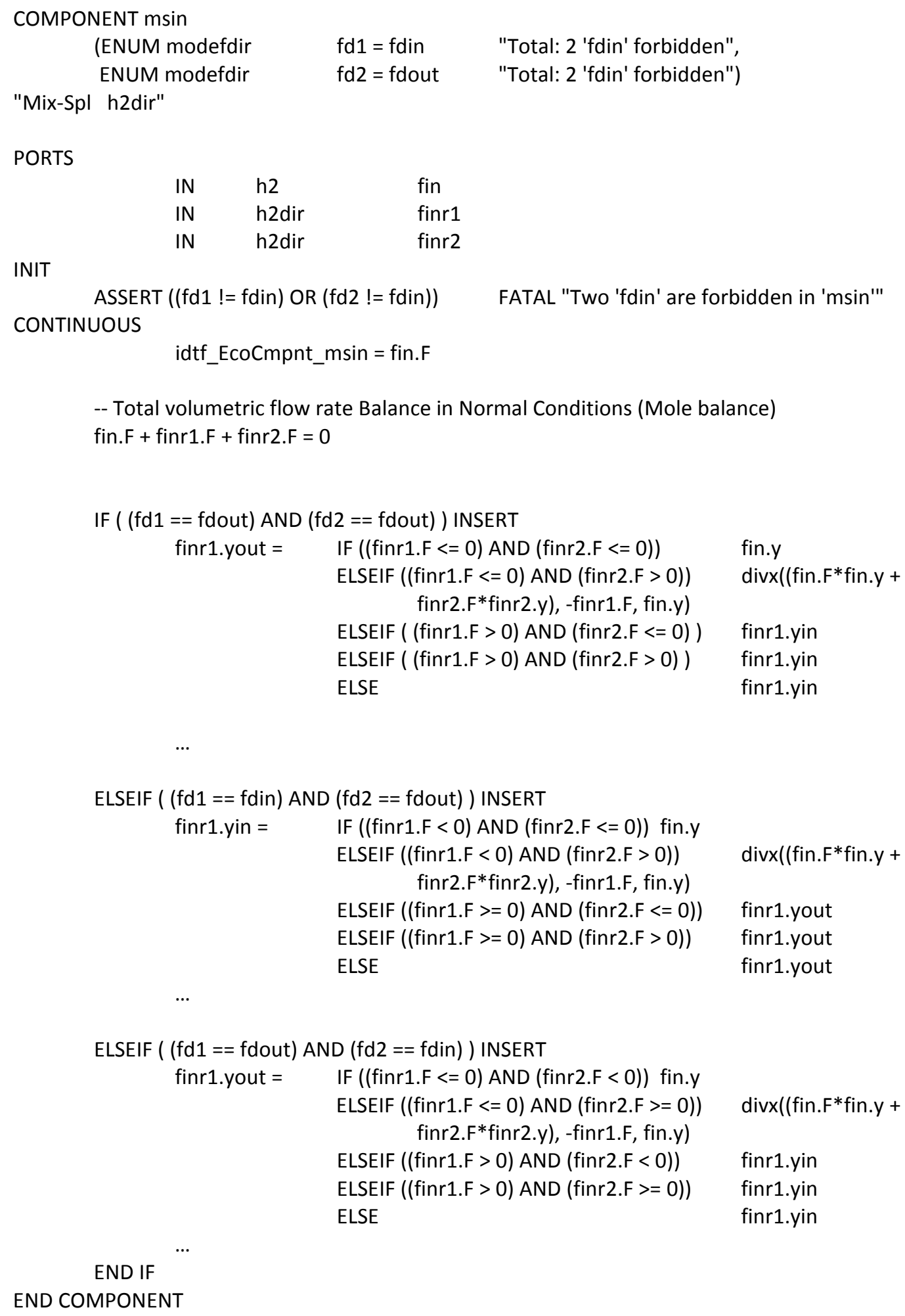




\begin{tabular}{|c|c|c|}
\hline \multicolumn{3}{|l|}{ COMPONENT msout } \\
\hline (ENUM modefdir & $\mathrm{fd} 1$ = fdout & "Total: 2 'fdout' forbidden", \\
\hline ENUM modefdir & $\mathrm{fd} 2=\mathrm{fdin}$ & "Total: 2 'fdout' forbidden") \\
\hline
\end{tabular}

"Mix-Spl h2dir"

PORTS

$\begin{array}{lll}\text { IN } & \text { h2dir } & \text { finr1 } \\ \text { IN } & \text { h2dir } & \text { finr2 } \\ \text { OUT } & \text { h2 } & \text { fout }\end{array}$

INIT

ASSERT ((fd1 != fdout) OR (fd2 != fdout)) FATAL "Two 'fdout' are forbidden in 'msout'"

CONTINUOUS

idtf_EcoCmpnt_msout $=$ fout.F

-- Total volumetric flow rate Balance in Normal Conditions (Mole balance)

finr1.F + finr2.F = fout.F

-- Total $\mathrm{H} 2$ vol. flow rate Balance in Normal Conditions ( $\mathrm{H} 2$ mole balance)

finr1. $F^{*}$ finr1. $y+$ finr2. $F *$ finr $2 . y=$ fout. $F^{*}$ fout.y

INVERSE(fout.y) fout.y $=\operatorname{divx}(($ finr1.F*finr1.y + finr2.F*finr2.y), fout.F, finr1.y)

-- Total mass flow rate Balance

finr1.F $*$ finr $1 . w+$ finr $2 . F^{*}$ finr2.w $=$ fout. $F^{*}$ fout.w

INVERSE(fout.w) fout.w $=\operatorname{divx}(($ finr1.F*finr1.w + finr2.F*finr2.w), fout.F,finr1.w)

-- Outlet Temperature

finr1.F $*$ finr1.T + finr2. $F *$ finr2.T $=$ fout. $F *$ fout. $T$

INVERSE(fout.T) fout.T $=\operatorname{divx}(($ finr1.F*finr1.T + finr2.F*finr2.T $)$, fout.F, finr1.T)

IF ( ( $\mathrm{fd} 1==$ fdout) AND ( $\mathrm{fd} 2==\mathrm{fdin}))$ INSERT

finr1.yout $=\quad \operatorname{IF}(($ finr1.F $<=0)$ AND $($ finr2.F $>=0)) \quad$ finr2.y

ELSEIF ( (finr1.F $>0$ ) AND (finr2.F $<0)$ ) finr1.yin

ELSEIF ( (finr1.F >0) AND (finr2.F $>=0)$ ) finr1.yin

ELSEIF ( (finr1.F < = 0) AND (finr $2 . F<0)$ ) fout.y

ELSE finr1.yin

...

ELSEIF ( $(\mathrm{fd} 1==\mathrm{fdin})$ AND ( $\mathrm{fd} 2==\mathrm{fdout})$ ) INSERT

finr1.yin = $\quad$ IF $(($ finr1.F $<0)$ AND $($ finr2.F $>0))$ finr2.y

ELSEIF ((finr1.F $>=0$ ) AND (finr2.F $<=0)$ ) finr1.yout

ELSEIF ((finr1.F $>=0)$ AND (finr2.F $>0)$ finr1.yout

ELSEIF ((finr1.F < 0) AND (finr $2 . F<=0)$ fout.y

ELSE

finr1.yout

ELSEIF ( ( fd1 $==f d i n)$ AND ( $f d 2==f d i n) ~)$ INSERT

finr1.yin = $\quad$ IF ((finr1.F < 0) AND (finr2.F > = 0)) finr2.y

ELSEIF ((finr1.F $>=0$ ) AND (finr2.F $<0)$ finr1.yout

ELSEIF ((finr1.F $>=0$ ) AND (finr2.F $>=0)$ ) finr1. yout

ELSEIF ((finr 1.F < 0) AND (finr $2 . F<0))$ fout.y

ELSE

finr1.yout

END IF

END COMPONENT 


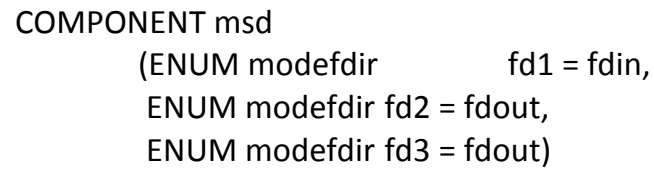




\section{"hc" port}

Variables declared to model the hydrocarbon port are shown in Table 4.5, as well as the corresponding port modelling equations. Three independent variables are needed as abovementioned to characterize hydrocarbon streams: $F_{H C}, W_{H C}, w^{H C}$.

\begin{tabular}{llll}
\hline Variable & Description & Units & Modifier \\
\hline$F_{H C}$ & Volumetric flow rate & $\mathrm{m}^{3} / \mathrm{h}$ & $\mathrm{SUM}$ \\
& & & \\
$W_{H C}$ & Mass flow rate & $\mathrm{kg} / \mathrm{h}$ & \\
$w^{H C}$ & Hydrocarbon molecular weight & $\mathrm{kg} / \mathrm{kmol}$ & EQUAL \\
$M_{H C}$ & Molar flow rate & $\mathrm{kmol} / \mathrm{h}$ & \\
$\rho_{H C}$ & Density & $\mathrm{kg} / \mathrm{m}^{3}$ & EQUAL \\
& & & \\
\hline
\end{tabular}

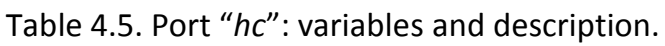

$$
\begin{aligned}
& W_{H C}=F_{H C} \cdot \rho_{H C} \\
& M_{H C}=W_{H C} / w^{H C}
\end{aligned}
$$

The port " $h c h 2$ " for mixtures gas-hydrocarbon is a straightforward combination of ports " $h 2$ " and " $h c$ ", without modelling the fraction of gas solved in liquid, or the hydrocarbon state either as gas, liquid or mixture.

\subsection{3- Selection of Boundary variables for Simulation}

According to EcosimPro ${ }^{\circledR}$ computational causality, values for all data variables (constants) and boundary variables (independent degrees of freedom) must be specified as inputs. Model inputs for the $\mathrm{H}_{2}$ network simulation are shown in Table 4.6. 


\begin{tabular}{|c|c|c|c|}
\hline Variable & Description & Units & Number \\
\hline$F_{H C}$ & Volumetric flow rate hydrocarbon & $\mathrm{m}^{3} / \mathrm{h}$ & 66 \\
\hline$W_{H C}$ & Mass flow rate hydrocarbon & $\mathrm{kg} / \mathrm{h}$ & 54 \\
\hline$w^{H C}$ & Molecular weight hydrocarbon & $\mathrm{kg} / \mathrm{kmol}$ & 54 \\
\hline$F$ & $\begin{array}{l}\text { Volumetric flow rate Normal conditions } \\
\text { gas stream (either directly or } \\
\text { through ForceFbnd in valves) }\end{array}$ & $\mathrm{Nm}^{3} / \mathrm{h}$ & 120 \\
\hline$\Delta R_{H 2}^{s p}$ & Specific $\mathrm{H}_{2}$ consumption & $\mathrm{Nm}^{3} \mathrm{H}_{2} / \mathrm{m}^{3} \mathrm{HC}$ & 16 \\
\hline$\Delta R_{L I G} s p$ & Specific light ends generation in volume & $\mathrm{Nm}^{3} \mathrm{LIG} / \mathrm{m}^{3} \mathrm{HC}$ & 16 \\
\hline$\Delta R_{w L / G}$ & Molecular weight of light ends generated & kg LIG/kmol LIG & 16 \\
\hline$k_{\text {gasHC }}^{S}$ & Gas solubility in hydrocarbon & $\mathrm{Nm}^{3} / \mathrm{m}^{3} \mathrm{HC}$ & 27 \\
\hline$k_{\alpha H 2 L I G}^{S}$ & $\begin{array}{l}\text { Relative solubility for } \mathrm{H}_{2} \text { and light ends in } \\
\text { gas and liquid streams }\end{array}$ & - & 27 \\
\hline$k_{\text {wLIG }}^{S}$ & $\begin{array}{l}\text { Relative solubility for light ends in gas } \\
\text { and liquid streams, that is, } \\
\text { distribution coefficient for light ends } \\
\text { molecular weight }\end{array}$ & - & 27 \\
\hline$\varepsilon$ & $\begin{array}{l}\text { Slack variable for non-linear constraints } \\
\text { regarding analysers }\end{array}$ & $\% 1 \mathrm{~mol}$ & 14 \\
\hline Total & & & 437 \\
\hline
\end{tabular}

Table 4.6. Model inputs for the $\mathrm{H}_{2}$ network simulation in EcosimPro ${ }^{\circledR}$.

In the assignment of computational causality (selection of boundary variables subset) following the physical causality from upstream to downstream, the number of degrees of freedom $\left(N_{\text {dof }}\right)$ introduced by each H2NET component is listed in Table 4.7, and briefly detailed next.

\begin{tabular}{ll}
\hline Component & Degrees of freedom introduced $\left(\boldsymbol{N}_{\text {dof }}\right)$ \\
\hline Fin, prdct & 3 (gas source: $\left.F, y, w^{L G}\right)$ \\
$\begin{array}{l}\text { Fout } \\
\text { Fin_hc }\end{array}$ & 0 \\
dep_inout & 3 (hydrocarbon source: $\left.F_{H C}, W_{H C}, w^{H C}\right)$ \\
reactors & 0 \\
shp, slp & 0 \\
$z$ & 0 \\
mixers & 1 (purge flow rate for every membrane unit) \\
\hline
\end{tabular}




\begin{tabular}{|c|c|}
\hline$s p l$ & 1 (gas flow rate for one outlet branch) \\
\hline$m \sin$ & 0 \\
\hline msout, msinout & 1 (gas flow rate for outlet branch) \\
\hline spl_[hc/hch2] & 1 (hydrocarbon flow rate for one outlet branch) \\
\hline dtOh2, dtOdir & 0 (gas flow rate, either forced or dummy) \\
\hline$v 3 v \_2$ in & -1 (mutually exclusive inlets) \\
\hline v3v 2out & 0 (mutually exclusive outlets) \\
\hline$v 3 S \bar{R}$ & 0 (mutually exclusive inlet/outlet) \\
\hline HP system & $\begin{array}{l}\text { in the splitter where the HP purge is generated, } \\
\text { it is mandatory to specify as bound not this } \\
\text { purge stream but the recycle stream. Although } \\
\text { the number of degrees of freedom is not } \\
\text { affected, provided that one flow rate is specified } \\
\text { as boundary per splitter component, once the } \\
\text { make-up is specified the HP purge is } \\
\text { straightforward known due to the material } \\
\text { balance (gas phase continuity) in a control } \\
\text { volume comprising the reactor and HP } \\
\text { separator, whereas the recycle stream needs to } \\
\text { be specified due to the lack of momentum } \\
\text { balances, being internal to this control volume }\end{array}$ \\
\hline $\begin{array}{l}\text { h2dir-subnetwork } \\
\text { (either closed- } \\
\text { loop or not) }\end{array}$ & $\begin{array}{l}\text { - } 1 \text { in total for each subnetwork, corresponding } \\
\text { to one outlet branch of an msout or msinout } \\
\text { component (due to global balance. It doesnot } \\
\text { occur with } h 2 \text {-subnetworks because spl } \\
\text { components always act as such, whereas in the } \\
\text { case of } h 2 d i r \text {, at least one } m \text { sout/msinout will } \\
\text { act as mixer necessarily, therefore reducing by } 1 \\
\text { the number of needed specified flow rates). For } \\
\text { each sub-net made of } h 2 d i r \text { ports, all outlet } \\
\text { ports for msout and msinout belonging to the } \\
\text { same } h 2 d i r \text { sub-net need to be specified as } \\
\text { bounds, except one of them }\end{array}$ \\
\hline h2dir-closed-loop & $\begin{array}{l}1 \text {; for each closed loop made up only of } h 2 d i r \\
\text { ports, one additional } h 2 d i r \text { flow rate must be } \\
\text { specified as bound due to recycle }\end{array}$ \\
\hline
\end{tabular}

Table 4.7. Degrees of freedom introduced by each component of the H2NET library.

Certain remarks are important to mention:

- $\quad d t O h 2$, dtOdir

The components enable to force zero flow rate, either in a gas stream with fixed direction for flow or in a gas stream with two allowed directions for flow. The $N_{\text {dof }}$ 
remains the same, $+1-1=0$, since they both decrease by 1 the $N_{\text {dof }}$, and also add 1 degree of freedom due to the auxiliary variable ForceFbnd.

- $\quad v 3 v 2$ in

The component, analogous to a mixer component with two mutually exclusive inlet streams, decreases by 1 the number of degrees of freedom $\left(N_{\text {dof }}\right)$ because one of the inlet streams has necessarily zero flow rate. This must be considered at the network level and is usually solved by assigning as boundary the outlet stream for v3v_2in, while leaving as explicit variables both inlet streams.

- $\quad v 3 v \_2 o u t, v 3 S R$

They decrease by 1 the $N_{\text {dof }}$, due to the mutually exclusive streams being one forced to zero flow rate. However, for both of them it would be necessary to specify one outlet stream flow, because only the other one is established from the material balance equations, so they are matched and the $N_{\text {dof }}$ is closed in the same component, $1-1=0$, and remains the same.

- $\quad$ high pressure HP system

In order to account for inventory balance in a closed control volume around the reactor comprising the high pressure HP separator and the entire HP system, it is mandatory to specify as bound in the splitter where the HP purge is generated not this purge stream but the recycle stream. The number of degrees of freedom is not affected provided that one flow rate is specified as boundary per splitter component, and follows from the aforementioned Table 4.7, but not all stream flows are equivalent or any subset feasible as boundary.

According to physical causality, following a upstream to downstream sequence for computation, and if all make-up streams to a consumer plant are known, then the HP purge is not independent and is established under pressure control. Alternatively, it can be fixed as bound the purge flow rate, whilst one of the makeup flow rates is under pressure control thus explicitly computed as a function of the boundaries. Sometimes purges from the HP system are closed, being easier to manage a zero flow for a boundary than for an explicit variable, since limits on decision variables are easier to handle than limits for constraint variables.

- h2dir closed-loops and h2dir subnetworks

The gas flow rates needed as degrees of freedom for cases h2dir closed-loop and h2dir subnetwork are illustrated in Figure 4.13. An example with a particular flow direction is shown in each case, although the aforementioned rules apply for any other network configuration and flow direction.

In the first schematic, bounds correspond to all the inlets as well as one outlet per splitter component according to the aforementioned rules, that is, the recycle stream is thus fixed and all the subnetwork outlets except one (determined from the material balance) are also fixed. It is equivalent if for spl_2 the alternative branch would have been specified instead of Fout_1. The same configuration although with both directions allowed for flow, second schematic, can be 
completely determined with the rules: all outlet streams for msinout/msout components except one, and one h2dir stream per closed-loop. It can be noticed that the functionality as mixer or splitter for $\mathrm{msin} / \mathrm{msout}$ components is the same as in the first schematic, however the rules apply even if the directions for flow change. In the third schematic only an h2dir subnetwork exists, but not a closedloop, thus having been specified all inlet flow rates and all outlet flow rates for msinout/msout components except one.
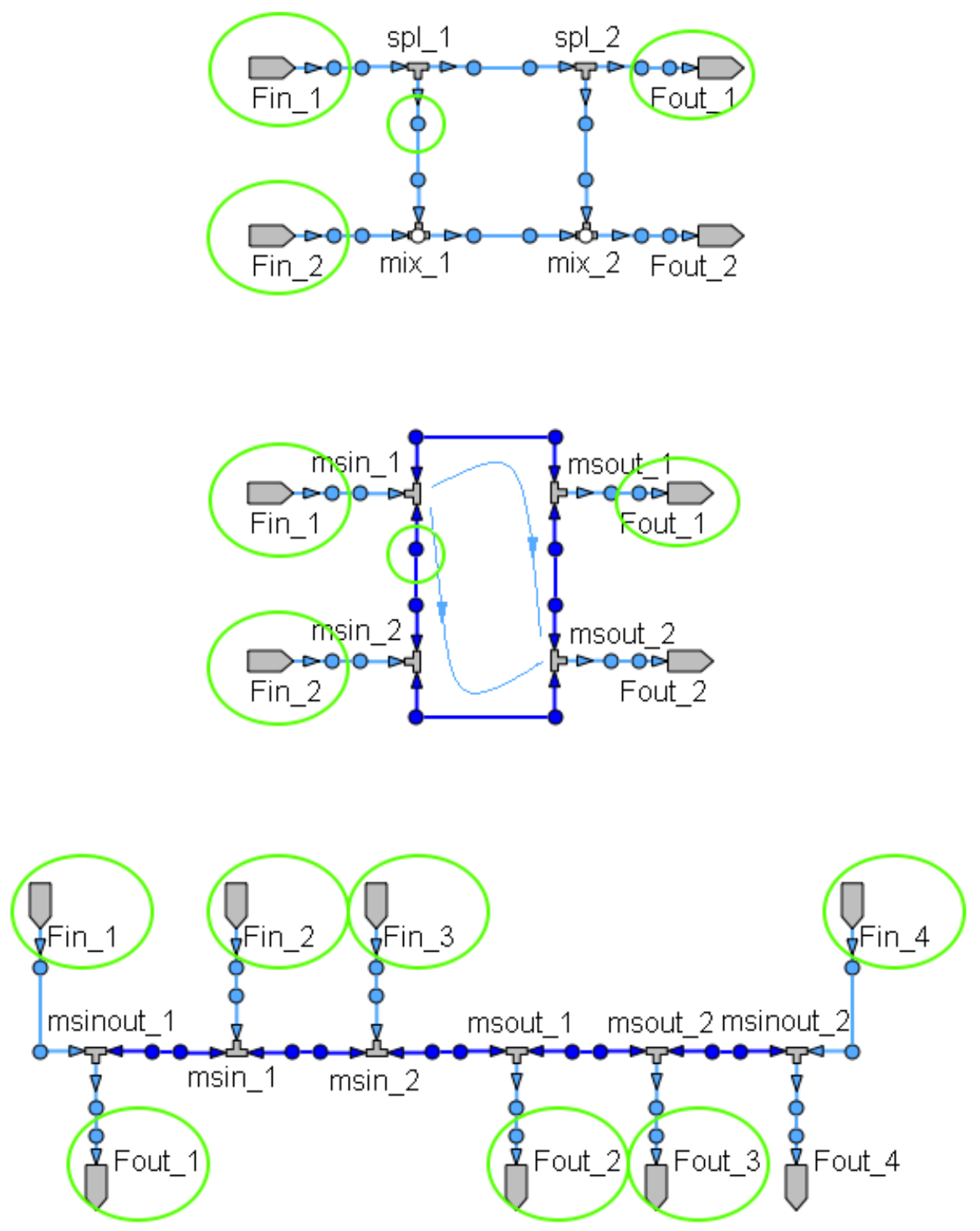

Figure 4.13. Examples of assignment of boundary flow rates according to physical causality. 


\subsection{4- H2NET Library Additional Features}

The code for both the data reconciliation and the optimal redistribution optimization problems has been generated quasi-automatically taking advantage of EcosimPro ${ }^{\circledR}$ capabilities and functions.

Provided EcosimPro ${ }^{\circledR}$ available functions and object oriented paradigm are understood, automatically generation of code is straightforward. According to object oriented modeling, a variable name is made up of the ordered names for all the aggregated objects in the hierarchy separated by points:

\section{ElementaryComponent.Port.PortVariable \\ ElementaryComponent.ComponentVariable \\ AgragatedComponent.ElementaryComponent.ComponentVariable}

It should be remarked that it is forbidden in EcosimPro ${ }^{\circledR}$ that component names contain the point '.' character. All the programmed functions to automatically generate the optimization problem structures are based on the EcosimPro ${ }^{\circledR}$ functions and characteristics listed in Table 4.8. Other functions as those for handling character strings have been used from a library programmed in Visual $\mathrm{C}++$ and developed in the Department. In the H2NET library, each elementary component and port type has been identified with a unique variable with the purpose of automatic code generation.

Based on the aforementioned functions, the structure for all problems solved by optimization techniques has been built automatically in the H2NET library, generating vectors for:

- decision variables;

- constraints;

- addends in objective function;

- bounds, for both decision variables and constraints;

- whether it is a measured flow rate or not and the tag name for the flowmeter when appropriate, for both decision variables (with EcosimPro ${ }^{\circledR}$ Bound category) and constraints (with EcosimPro ${ }^{\circledR}$ Explicit category) which are flow rates;

- whether a valve exists or not and the tag name for the valve when appropriate, for both decision variables (with EcosimPro ${ }^{\circledR}$ Bound category) and constraints (with EcosimPro ${ }^{\circledR}$ Explicit category) which are flow rates;

A trivial example of how to collect variable names for specific $\mathrm{H}_{2}$ consumption terms for reactors is shown in Fig. 4.14. 
Function

getNumberVars()

getVarName()

getVarCategoryStr()

getVarAlias()

existsVariable()

compareStringsAtEnd()

concatStrings()

truncateStringsAtEnd()

\section{Description}

Returns the total number of model variables

Returns the name of a model variable according to its index

Returns the category (Bound, Explicit, Derivative, etc.) of the variable specified as argument

For a variable, returns the name of the root variable which is equivalent to it according to the model, that is, completely interchangeable and exactly equal

Returns True if a variable exists

Returns True if the $2^{\text {nd }}$ string is contained exactly at the end of the $1^{\text {st }}$ string

Concatenate two character strings

Truncate a character string which is at the end of another one; wild cards are feasible: ".*.*"

Table 4.8. EcosimPro ${ }^{\circledR}$ functions and additional features.

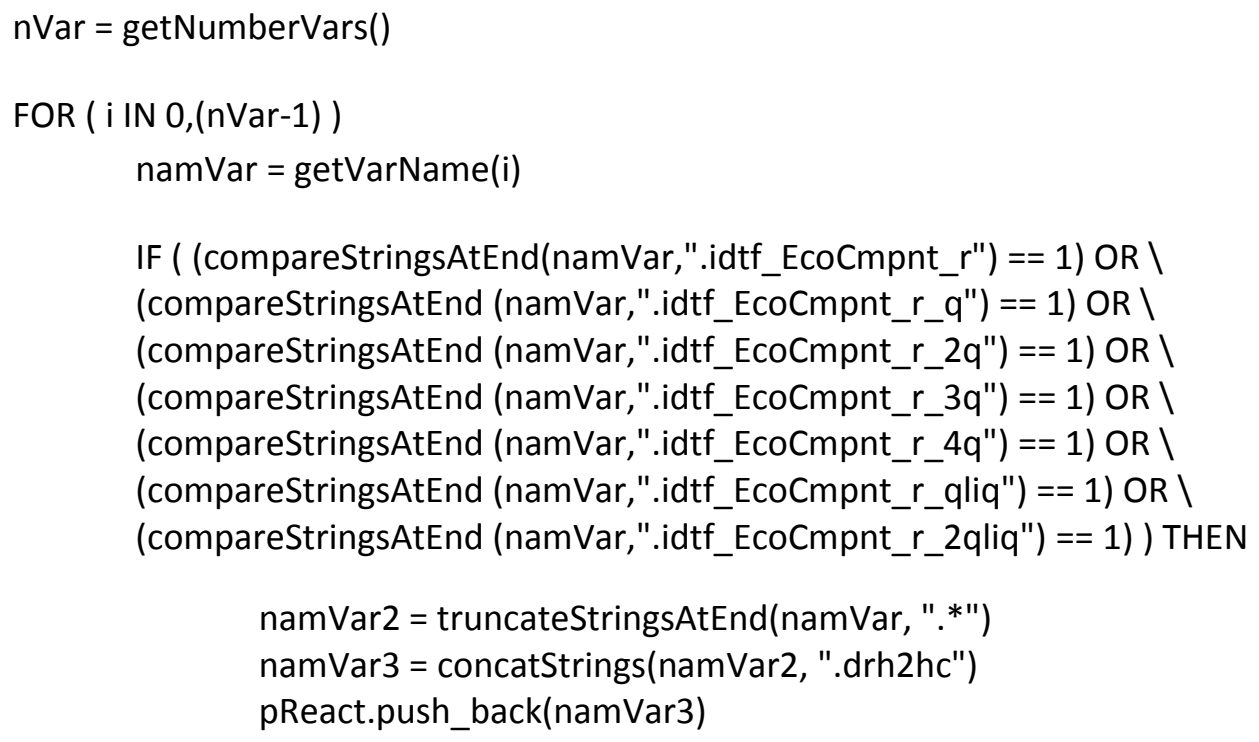

Figure 4.14. Example of the generation of variable names vectors automatically. 


\subsection{5- Configuration, Data Acquisition and Simulation}

The procedure for data acquisition and model simulation is briefly stated in Fig. 4.15, and explained next according to the sequence of steps for configuration, data acquisition and experiment simulation.

1. CONFIGURATION, both in Exce ${ }^{\circledR}$ and EcosimPro ${ }^{\circledR}$, once at configuration stage.

- Plant data from the SCADA can be read directly from Exce ${ }^{\circledR}$, just by providing the tag name, which can be previously selected from a menu in Excel ${ }^{\circledR}$, and the corresponding time. The categories of tags for all the needed measurements are listed in Table 4.9.

During configuration of the tool, tags for all measured variables needed are selected in Excel $^{\circledast}$. Besides, a macro is developed in Excel ${ }^{\circledR}$ to generate a suitable list for use in EcosimPro ${ }^{\circledast}$, where all the SCADA tag names are converted into EcosimPro ${ }^{\circledR}$ feasible names.

- When the $\mathrm{H}_{2}$ network model is graphically built, all configuring parameters regarding measurement tags are assigned, selected from a drop-down menu in EcosimPro ${ }^{\circledast}$. The needed parameters and data to configure the flowmeter component "Fl" are shown in Fig. 4.16 as an illustrative example, although also other H2NET components need a similar configuration to be linked with SCADA tags.

Also at the modelling configuration stage, a list is generated in EcosimPro ${ }^{\circledR}$ relating all the EcosimPro ${ }^{\circledR}$ variable names with all the already configured SCADA tags. This list is the link enabling to associate read values of average and standard deviations of tags with EcosimPro ${ }^{\circledR}$ variable names. The Excel ${ }^{\circledR}$ file with the list establishing the link between model names and SCADA tags can be seen in Fig. 4.17.

2. DATA ACQUISITION, performed in Excel ${ }^{\circledR}$.

- For every acquired data set, first plant data are read from the SCADA in Excel ${ }^{\circledR}$, and then a file is written in Excel $^{\circledR}$ for being use as input data file in EcosimPro ${ }^{\circledR}$.

- Reading of Data from the SCADA: for all tags already configured in Excel ${ }^{\circledR}$, process data of average and standard deviation are straightforward read in an Excel ${ }^{\circledast}$ sheet using SCADA functions, according to dates specified by the user.

- Input file writing: with an Exce $^{\circledR}$ routine which is based on the list EcosimPro ${ }^{\circledR}$ model names-SCADA tags generated in the modelling configuration stage, a text file is automatically written in EcosimPro ${ }^{\circledR}$ format, where all model variables linked to a measurement are assigned the corresponding measured value. The 
input text file for the EcosimPro ${ }^{\circledR}$ model, with readings for all SCADA tags corresponding to a snapshot, is shown in Fig. 4.18.

3. EXPERIMENT SIMULATION, performed in EcosimPro ${ }^{\circledR}$.

- For every experiment run, first plant data are restored from file, then data treatment is executed, then boundary variables are initialized and finally the model is simulated.

- Plant data are restored from file: for all meters and valves, average and standard deviation values are read from file in EcosimPro ${ }^{\circledR}$.

- Data treatment: it is performed in EcosimPro ${ }^{\circledR}$ according to certain rules, in the initialization stage of the simulation. Also units shut down are identified. The scheme followed for data treatment can be seen in Fig. 4.19, and is explained there.

- Boundary variables initialized: initialization with measured values. It is commented at the end of this epigraph, after the data treatment.

- Model simulation: equations in the Continuous block are computed.

Tags for all the needed measurements are automatically classified, ordered and written to a file according to EcosimPro ${ }^{\circledR}$ syntax from Excel ${ }^{\circledR}$. The categories are listed in Table 4.9.

\begin{tabular}{llc}
\hline TAG & Variable Description & No tags \\
\hline tag_OP & $\begin{array}{l}\text { Valve opening, for gas and hydrocarbon streams } \\
\text { Hydrocarbon volumetric flow, compensated or } \\
\text { tag_Vhc } \\
\text { not for Standard conditions }\end{array}$ & 214 \\
tag_WhC & $\begin{array}{l}\text { Hydrocarbon mass flow rate, compensated or } \\
\text { not for Standard conditions }\end{array}$ \\
& $\quad$ Gas flow rate at Normal conditions \\
tag_F & Gas pressure \\
tag_P & Gas temperature \\
tag_T & Analyser or minimum relation $\mathrm{H}_{2} / \mathrm{HC}$ & 176 \\
tag_Al & & 121 \\
TOTAL & & 163 \\
& & $14+10$ \\
\hline
\end{tabular}

Table 4.9. Categories for all measurements used from Petronor ${ }^{\circledR}$ SCADA. 


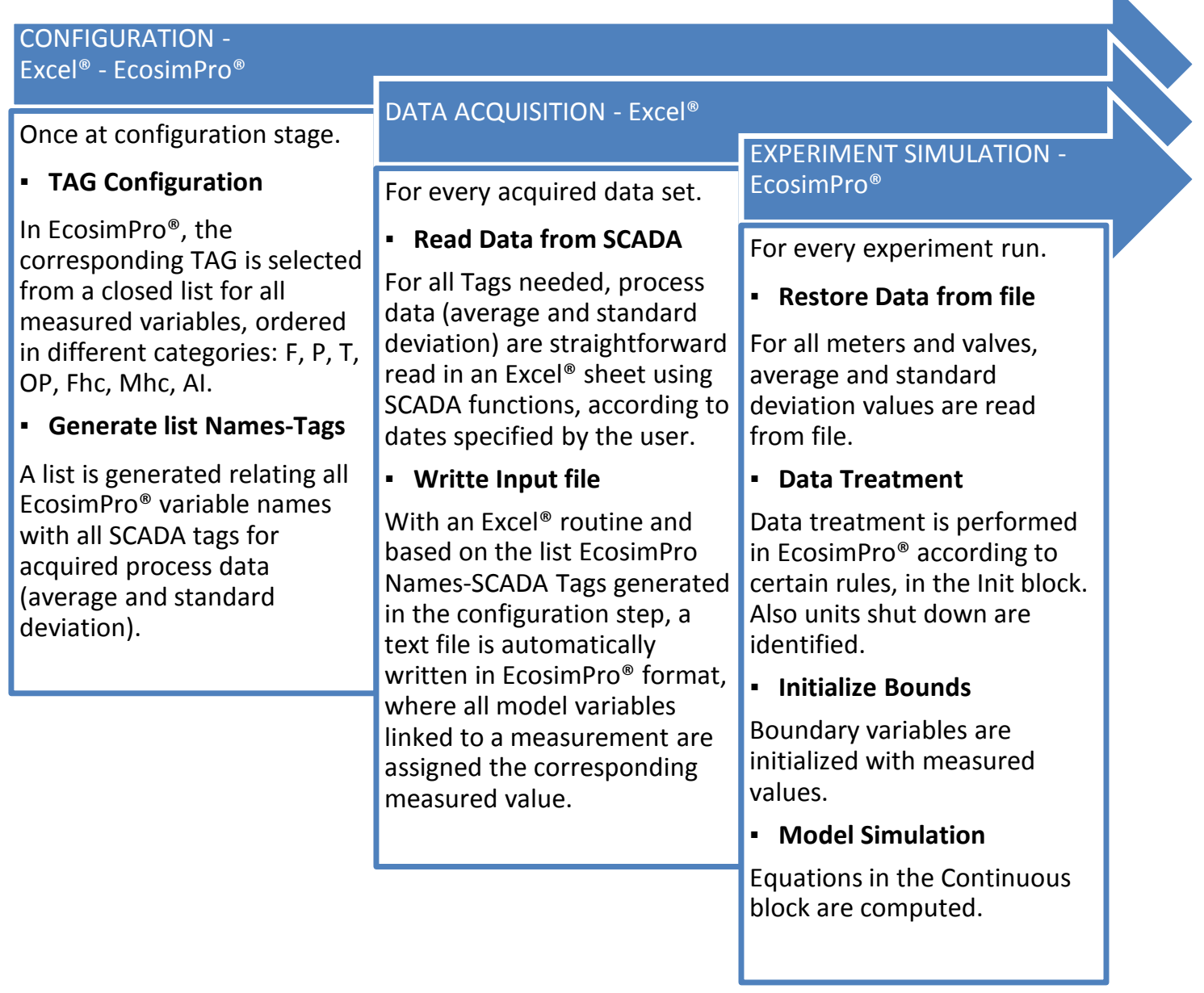

Figure 4.15. Schematic layout of steps for data acquisition and experiment simulation.

As an illustrative example, the needed parameters and data to configure the flowmeter component " $F l$ " are shown in Fig. 4.16. The parameters to establish the link with SCADA tags in order to read real plant variables are selected from a drop-down menu. Besides, data corresponding to design conditions, limits to perform the data reconciliation, limits to perform the optimal redistribution, as well as weighting factors can be introduced in the editor. 


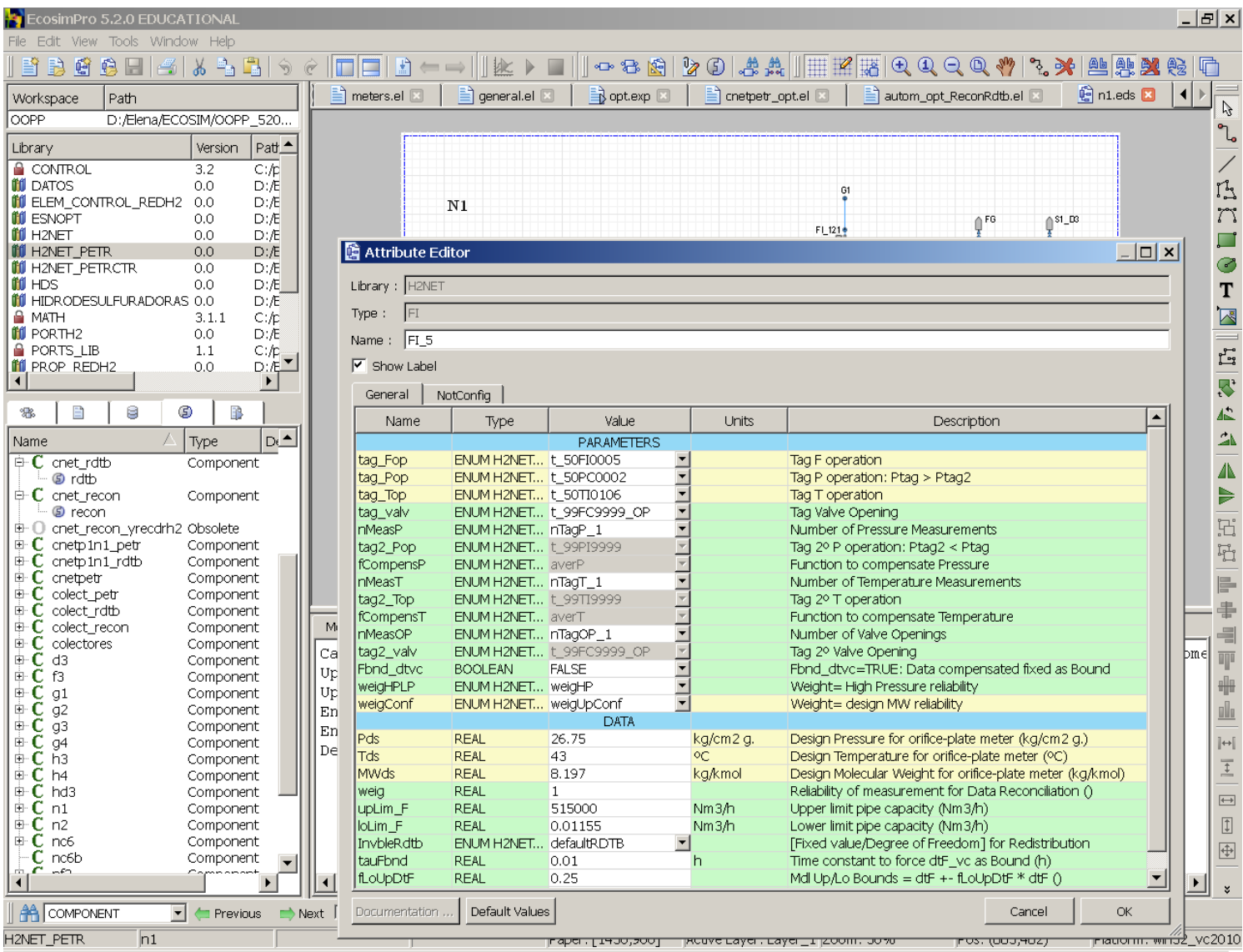

Figure 4.16. Flowmeter configuration, with tags read from the Petronor ${ }^{\circledR}$ SCADA and other design parameters and limits.

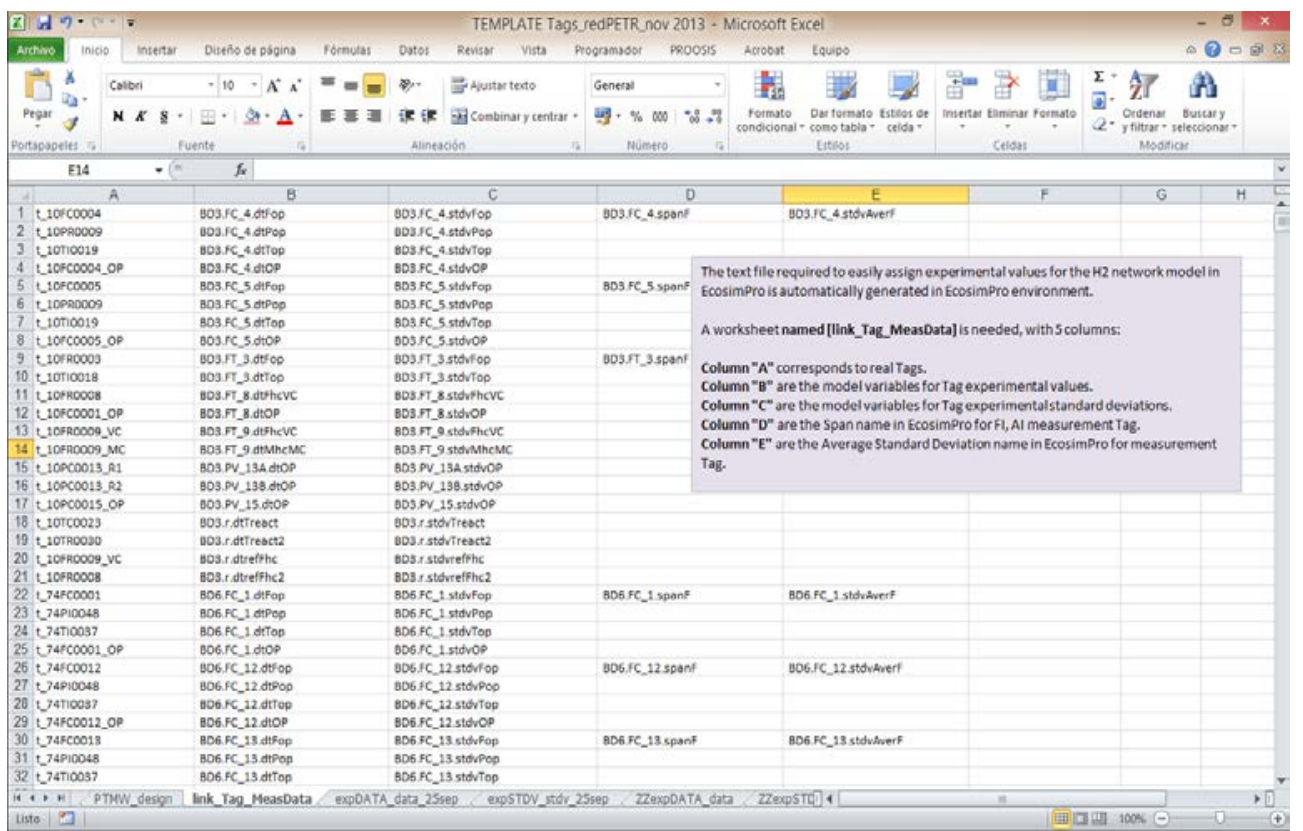

Figure 4.17. Excel ${ }^{\circledR}$ file with the link between model names and SCADA tags. 


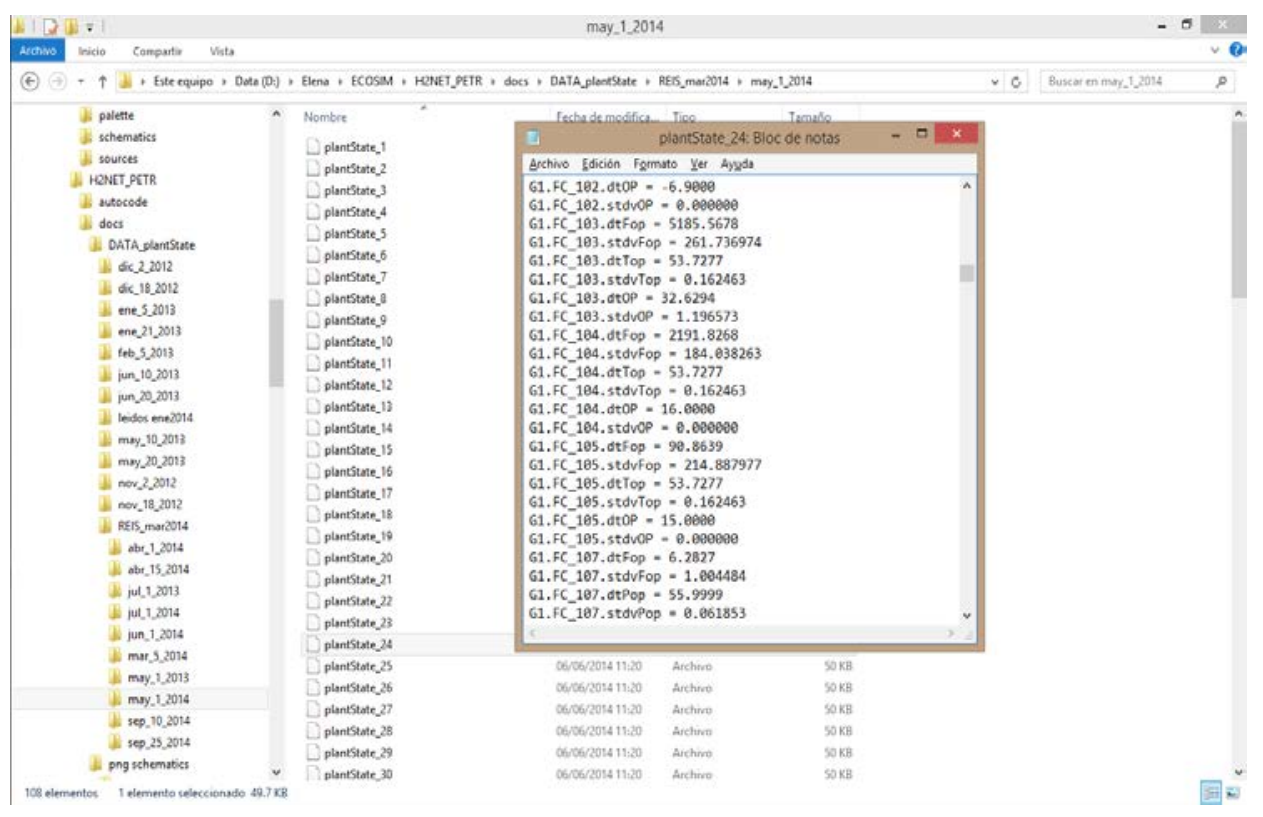

Figure 4.18. Text file with readings for all SCADA tags corresponding to a snapshot.

\section{Data Treatment}

Data treatment, before any assignment, is performed according to: data type (pressure, temperature, analyser, gas flow rate at Normal conditions, hydrocarbon volumetric flow rate Fhc, hydrocarbon mass flow rate $M h c$ ), experimental raw measurement (dtMeas), standard deviation for measurement $(S t d v)$, and valve opening $(d t O P)$. In the data treatment, certain faulty measurements can be detected and a zero weight assigned accordingly due to the lack of reliability when performing the data reconciliation. No weight is set for hydrocarbon measurements since they are not reconciled.

It should be noted that no treatment according to Stdv is done for gas flowmeters, because it is not frequent to have a wrong measurement "frozen" as is for analysers, and besides it would be difficult to specify a threshold: when being controlled or due to a closed valve, Stdv would be close to zero without meaning wrong measurement. 


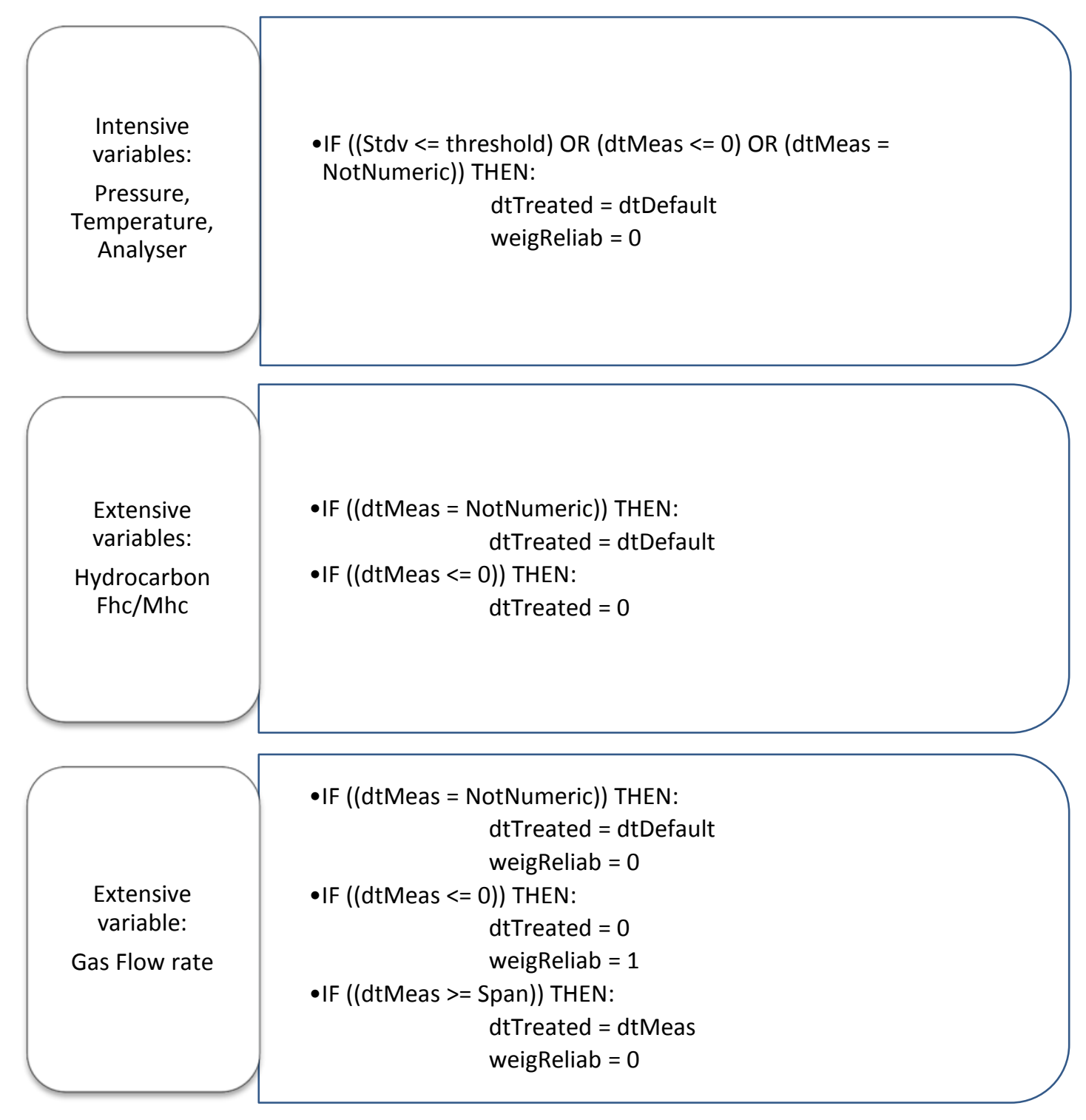

Figure 4.19. Automatic Data Treatment.

Regarding initialization of model with the already treated measurements, different priorities are assigned to the Initialization blocks of the different EcosimPro ${ }^{\circledR}$ components in order to be executed, taking advantage of this EcosimPro ${ }^{\circledR}$ feature. It is determining for a proper model simulation, especially regarding the on/off plant state, because measured values are assigned to model variables in local meter Fl/Al components, whereas the on/off state is determined in the reactor components, and the state spread through the whole network topology because it is needed for a proper computation in other components as the separators. The order of priorities for the initialization blocks of the components, together with an explanation, is stated below: 
1. Meter components: $F I, A l, F l d, F l \_h c, t u b$.

First, each measured value is assigned to a model variable. It is accomplished locally in the INIT block of the corresponding meter component. The value remains for boundary variables, although is overwritten for explicit variables when the simulation is run.

2. Reactors.

Plant state on/off is determined depending on measured values for reactor temperatures and measured values for plant inlet hydrocarbon flow rates in the reactor component. This plant state is spread through the whole network by means of the "hch2" port; thanks to the EQUAL modifier used in the declaration of the "state" port variable and the component equations where the "state" variable for the outlet port is made equal to that of the inlet port, all "state" variables for a certain consumer plant are equivalent. Reactor terms for $\mathrm{H}_{2}$ consumption and light ends generation are fixed depending on the plant state: between lower/upper limits when state is on, zero when state is off.

3. Separators.

Coefficients for the solubility equilibrium are fixed depending on the plant state: between lower/upper limits when state is on, zero when state is off.

\section{4- CONCLUSIONS}

Plantwide simplified models for the $\mathrm{H}_{2}$ distribution network of an oil refinery have been developed and validated, intended for steering process operation towards optimality in a decision-support system for the on-line optimal management.

It is worthy to remark the following features:

- Being first-principles models, prediction capabilities are reliable.

- Most of the proposed parameters are physically meaningful, in particular those for reactors and separators, therefore aiding in process understanding and useful for comparison purposes among similar units.

- The model is simple enough to be on-line calibrated and updated.

- The gas stream model with two allowed directions for flow provides certain flexibility in the optimization regarding topological decisions, whereas entailing a simple approach as compared to a hybrid modelling with binary decision variables.

An overview of parameters sensitivity and identifiability is remarked below, condensing process insight. 
- A systematic sensitivity and identifiability analysis has not been performed. The developed model is the simplest expression and introduces the minimum number of parameters to account for all the phenomena regarding $\mathrm{H}_{2}$. Identifiability issues have been addressed with narrow limits in parameters when appropriate according to process knowledge and operating conditions, thus reducing the effects of overparameterization.

Regarding the HP system, the model is formally overparameterized, although in practice consistent results are obtained for most of the streams due to: i) significant redundancy (\%) in flow measurements; ii) the existence of on-line analysers in both big and medium size plants; iii) the bounded variability for the generated light ends molecular weights $\Delta R_{w L I G}$, with a reduced range with respect to the expected in operation; iv) narrow bounds for certain parameters according to process knowledge and operating conditions: ratio light ends generation to $\mathrm{H}_{2}$ consumption $\Delta R_{L I G H 2}$, and relative solubility coefficients $k_{\alpha H 2 L G G}^{S}$ and $k_{\text {WLIG }}^{S}$ for $\mathrm{H}_{2}$ and light ends distribution between phases.

Regarding the MP/LP systems, the model is overparameterized due to the lack of on-line analysers for $\mathrm{H}_{2}$ purity and stream molecular weight, together with a smaller degree of flowmeters redundancy than in the HP system; moreover, process variability in composition is significantly higher as compared with the HP system, according to historical laboratory data. Nevertheless, the gas outlet flow rate has a strong influence over the solubility equilibria achieved, being measured in spite of compensation uncertainty and potential drift errors, thus providing a valuable reference.

The model could be enhanced, although highest modelling efforts are probably not justified according to its intended purpose.

- A model for reciprocating compressors could be studied, in order to ascertain whether they can provide a useful estimation of the driven gas molecular weight and flow rate as a function of measured variables, i.e. whether the sensitivity is enough for the said purpose. Reciprocating compressors are used in the joint make-up stream, which comprises important decision variables with a certain degree of uncertainty in the measured flow rates. A model for centrifugal compressors aiming for the estimation of gas molecular weight is not very interesting because they are employed in recycle streams where an on-line analyser is available for all the significant consumer plants.

- With the purpose of allowing increased process variability in certain model parameters while at the same time not compromising robustness and consistency, correlations on a coarse level as a function of measurable variables with significance could be studied. In particular, correlations for: i) molecular weight of light ends generated in catalytic-reforming producer plants as a function of both reactor temperature and feed quality; ii) relative solubility coefficients in the HP 
separator for important consumer plants as a function of both operation temperature and product composition, to improve the accuracy in the equilibrium estimation.

- Since off-line historical measurements from laboratory analysis could be used as reference, at least for the most important plants, as well as a complete set of values for all variables corresponding to design conditions, no other model verification has been carried out regarding the simulation for a consumer plant, for example against the commercial Hysys ${ }^{\circledR}$ simulation software. 



\section{CHAPTER 5}

\section{Hydrogen Network DATA ReCONCILIATION}

\section{1- INTRODUCTION}

Data reconciliation is the technique to estimate plant state from available on-line measurements based on a process model, i.e. the estimation of values of process variables that are consistent with their constraining mass and energy balances. The purpose of data reconciliation is to guarantee accuracy, consistency, and robustness in process state estimation based on the underlying conservation laws, irrespective of process disturbances, measurement noise, measurement drifts, outliers and unreliable measurements, reducing these errors by making use of redundancies in process data, while at the same time enabling to update certain unknown model parameters. Data reconciliation is a first stage needed for subsequent applications that use first-principles models like real-time optimization RTO, key performance indices KPI computation, NMPC, soft-sensors and process monitoring, in which accurate adaptive models and reconciled data are needed. The simultaneous reconciliation and update of parameters of a first-principles model can be achieved using an optimization framework that exploits physical and analytical redundancy of information. Currently, many steady-state data reconciliation applications are running on-line in operating refineries.

The data reconciliation approach is meaningful when redundancy exists in the available on-line measurements, trying to ascertain the most accurate and consistent estimation regardless of measurement noise, measurement drifts and outliers, which are common in industrial practice. It is assumed that a process model, in general stationary and consisting of conservation laws, relates all process states $\boldsymbol{x}$, measurements $\boldsymbol{y}$, and model parameters $\theta$.

$$
f(x, y, \theta)=0
$$

The estimation is formulated as the solution for the mathematical optimization problem of minimizing the model-measurement deviations, subject to model constraints and the corresponding bounds on variables, where $\boldsymbol{u}$ is the vector of inputs and $\sigma_{i}$ the measurement $i$ standard deviation. 


$$
\begin{gathered}
\min _{u, \theta} J=\sum_{i \in \text { Measurements }}\left(\frac{y_{i}-y_{i}^{\text {measured }}}{\sigma_{i}}\right)^{2} \\
\text { s.t. } \quad f(x, y, \theta)=0 \\
u^{l o} \leq u \leq u^{u p} ; x^{l o} \leq x \leq x^{u p} ; \quad \theta^{l o} \leq \theta \leq \theta^{u p} ; y^{l o} \leq y \leq y^{u p}
\end{gathered}
$$

It is well known that if measurement noise is white, that is, normally (Gaussian) distributed, centred in the true process value and independent, then minimization of the weighed sum of square errors leads to the best unbiased estimation (weighed least square WLS estimation). Nevertheless, in industrial practice gross errors are common: systematic errors (bias), outliers, drift errors depending on the measurement magnitude or other related variables, all of them referred to as gross errors which are non-random and non-gaussian. Non-random errors can be due to different reasons: improper sensor installation, lack of maintenance work, improper calibration. Theoretically, unbiased estimates cannot be guaranteed with the WLS technique in the presence of non-random errors.

As an alternative to the WLS estimator, where errors are penalized in a quadratic form thus being the solution significantly influenced by gross errors, robust estimators as the Fair or the Welsch functions have been proposed in the literature (Arora and Biegler, 2001). The error contribution to the cost function is asymptotically limited for big deviations potentially affected by gross errors, and as a consequence large errors do not influence negatively the reconciled results.

When a dynamic model has been identified, dynamic data reconciliation can be performed taking advantage of time series in measurements to facilitate robustness and consistency. However, the increased model integration time will make the solution expensive in terms of computation time.

\section{2- STATE OF THE ART}

Kuehn and Davidson (1961) recognized the benefit of using estimates of process measurements that are consistent with constraining mass and energy balances. The estimation of the values of unmeasured variables from measured process data is termed coaptation by Mah et al. (1976). Classification of variables to determine which unmeasured ones can be estimated and which measured ones are available for improvement by reconciliation is shown by Stanley and Mah (1981). Several good examples of simultaneous estimation and reconciliation of process variables are shown by Tamhane and Mah (1985). The problem of estimating the variables involved in a chemical process, subject to linear balance equations, has been considered by several authors, in particular Romagnoli and Stephanopoulos (1980); Crowe, García Campos, and Heymak (1983). For a complete account see Romagnoli and Sánchez (2000). Basic issues are whether the estimation of an observed value can be improved 
by using the other measurements (redundancy), an unobserved value is estimable from the observed ones (determinability) and whether an observed value is a gross error. Surveys on the topic have discussed improvements to the methods and the different applications of data reconciliation (Crowe, 1996; Dochain, 2003; Puigjaner and Heyen, 2006). Comprehensive books were also published (Narasimhan and Jordache, 2000; Romagnoli and Sanchez, 2000).

For dynamic systems, the data reconciliation problem shares many characteristics with state and parameter estimation. Almasy (1990) described a technique for dynamic data reconciliation using linear balancing equations to reconcile measured states, and Liebman et al. (1992) demonstrated that nonlinear balance equations could be reconciled efficiently using NLP techniques. For an industrial application of a large-scale dynamic data reconciliation strategy, Soderstrom, Edgar, Russo and Young (2000) can be seen. Moving-horizon methods for state estimation are discussed by Muske and Edgar (1997), and implemented industrially for a polymerization process by Russo and Young (1999).

The application of steady-state data reconciliation on a real-time basis has limitations when process dynamics become significant. In some cases, reconciliation produces estimates with a larger variance than measurement errors and using reconciled data could yield worst results than using the measurements directly (Almasy, 1990). On the other hand, using a complete and exact model of the process for dynamic data reconciliation is feasible, however such a complete phenomenological dynamic model is complex to develop and calibrate, requires maintenance, and an improper representation of the process could lead to biased estimates (Dochain, 2003). Comparisons of data reconciliation approaches based on different submodels or averaging techniques are respectively proposed by Lachance, Desbiens, and Hodouin (2006) and Bagajewicz and Jiang (2000).

Bagajewicz (2006) extended the concept of economic value of precision to include the effect of induced bias, that is, he obtained the economic value of accuracy. Such economic value allows one to determine the economic gain when one makes use of accuracy-improving methods such as installing data reconciliation software, performing instrumentation upgrade or implementing better maintenance. The value of economic gain, in turn, helps one determine whether it is worthwhile to perform such investments, i.e., determine the balance between economical gain and the investment cost. Bagajewicz et al. (2005) and Bagajewicz (2006) provided examples on the economic value of performing data reconciliation and on the economic value of instrumentation upgrade.

Manenti, Grottoli and Pierucci (2011) deal with the integrated solution of different modelbased optimization levels to face the problem of inferring and reconciling online plant measurements practically, under the condition of poor measure redundancy simultaneous integration of different optimization levels: (i) the data reconciliation based on a detailed process simulation; (ii) the introduction and estimation of certain adaptive parameters, to match the current process conditions as well as to confer a certain generality on it; and (iii) the use of a set of efficient optimizers to improve plant operations. The online feasibility of the proposed CAPE solution is validated on a large-scale sulfur recovery unit (SRU) of an oil refinery. 
Furthermore, one of the major focuses for research in data reconciliation has been to use data or equation residuals for the detection of gross errors and outliers in process data. Chen and Romagnoli (1998) present a strategy for simultaneous dynamic data reconciliation and outlier detection.

\section{3- MOTIVATION}

Data reconciliation in the $\mathrm{H}_{2}$ network is a challenging problem due to the difficulty of an accurate plant state estimation; uncertainty is mainly caused by:

- A great variability in operating conditions and in hydrogen consumption in HDT hydrotreating plants, mainly influenced by: i) feedstock sulphur content; ii) desired grade for naphtha/diesel, with low or high sulphur product specification according to its final use as either transport fuel or heating fuel; iii) the type of hydrocarbon processed, and specially the light-cyclic-oil LCO content, due to double-bounds leading to higher $\mathrm{H}_{2}$ consumption.

- Lack of on-line measurements for gas $\mathrm{H}_{2}$ purity and molecular weight MW. In general, on-line complete quality analysis of products is difficult due to mixtures complexity and also economically demanding due to installation and maintenance costs of analysers, thus not often economically justified in process industries. Therefore, online analysers are scarce.

- The use of orifice-plate differential pressure flow meters for gas streams in spite of being error prone because it is the cheapest available technology, also commonly used for similar applications in other process industries. Drift errors are present due to: i) process variability, with different $[P, T, M W]$ conditions in operation from those corresponding to design/calibration; ii) the differential pressure transmitter, when flow rate is up/low in the scale; iii) inaccurate compensations, i.e. flowmeters where no pressure measurement is available but just downstream or upstream a valve, flowmeters where no temperature measurement is available.

- Furthermore, another source contributing to uncertainty in a great extent is the fact that gas molecular weight MW experiences significant variations for small changes on the light ends composition, due to the low value of $\mathrm{H}_{2}$ molecular weight $w$ as compared to those of the main impurities, $\mathrm{CH}_{4}, \mathrm{C}_{2} \mathrm{H}_{6}$, and $\mathrm{C}_{3} \mathrm{H}_{8}$, whose $w$ are $2,16,30$ and 44 respectively. This is an important difference as opposed to other gas networks as natural gas networks, where gas composition can be assumed constant.

- In certain cases, improper instrument installation or maintenance is also responsible for systematic errors. 


\section{4- DATA RECONCILIATION APPROACH}

The approach to deal with uncertainty is the following:

- Instruments with gross errors, usually persistent in time, have been disregarded;

- A simplified solubility model have been proposed to determine the item (medium and low pressure MP/LP purges) contributing less to $\mathrm{H}_{2}$ make-up requirements, while providing consistent estimates. Losses in the medium and low pressure MP/LP purges represent typically about $10 \%$ of the total $\mathrm{H}_{2}$ production, as can be seen in Figure 5.1.

- A data reconciliation with optimization techniques is performed on-line to estimate $\mathrm{H}_{2}$ consumption in reactors and plant states according to flow meters $(\mathrm{FI})$ and $\mathrm{H}_{2}$ purity analysers (AI) measurements, in order to achieve a reliable and consistent estimate of the network state.

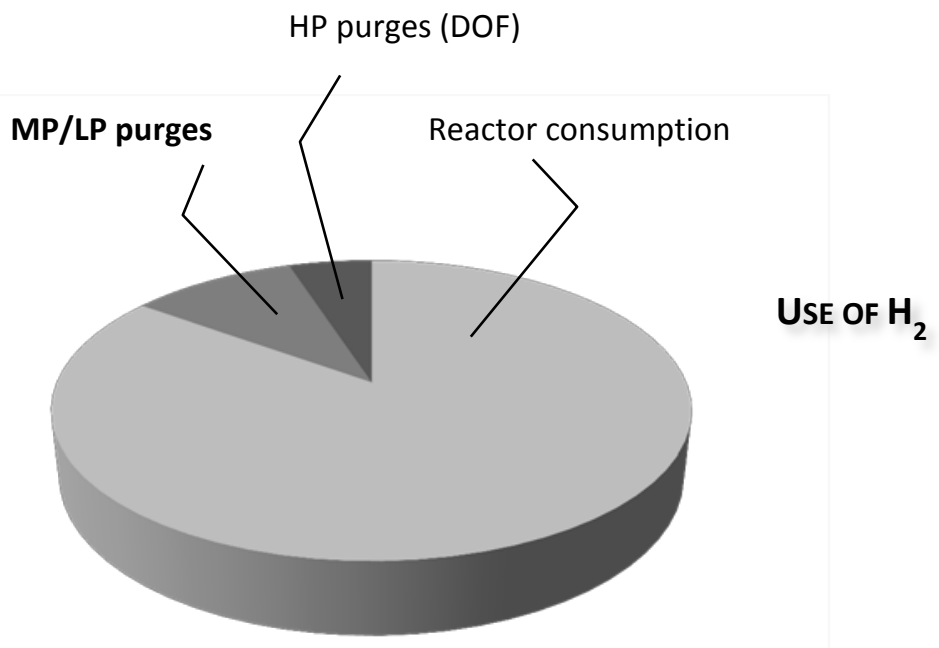

Figure 5.1. Purges in the MP/LP systems represent about $10 \%$ of the total $\mathrm{H}_{2}$ production.

\subsection{1- Systematic errors in practice}

In the hydrogen network problem, experience for different periods of operation along years has shown the presence of potentially systematic and drift errors. When local redundancy in a node exists, and the residual $(r)$ sign and also approximately magnitude remain constant along time, being the residual meaningful as compared to the flow rates, the non-random nature of the measurement errors is pointed out.

$$
\sum_{i}^{I N} F_{i}=\sum_{j}^{\text {oUT }} F_{j}+r \quad \forall \text { node }
$$


This is a somewhat frequent situation, and has been used to disregard by inspection flowmeters affected by gross errors. Systematic errors can not be explained because of the uncertainty due to compensation for gas $\mathrm{MW}$ in operation, as they also occur in splitter nodes where the composition is identical and affect the three compensated flow rates involved with known direction: this systematic error can not be cancelled irrepective of the value assigned to the gas MW.

Another source for drift errors is when the pressure transmiter of an orifice-plate flowmeter is measuring close to the upper range. This is probably the case of the drift error illustrated in Figure 5.2. Only green and blue lines are meaningful, the rest are negligible and are shown only for the purpose of corroboration. They correspond to two flow meters which are redundant when the rest of flow rates linked to the header are zero, as well as the opening for one relief valve also shown. Both raw measurements overlap when the absolute value for the measurement is not high, but a drift error appears when the measured flow rate reaches a high value, close to the upper range for one of the flowmeters. Another example is shown in Figure 5.3, with two redundant flowmeters and the valve opening available in the stream.

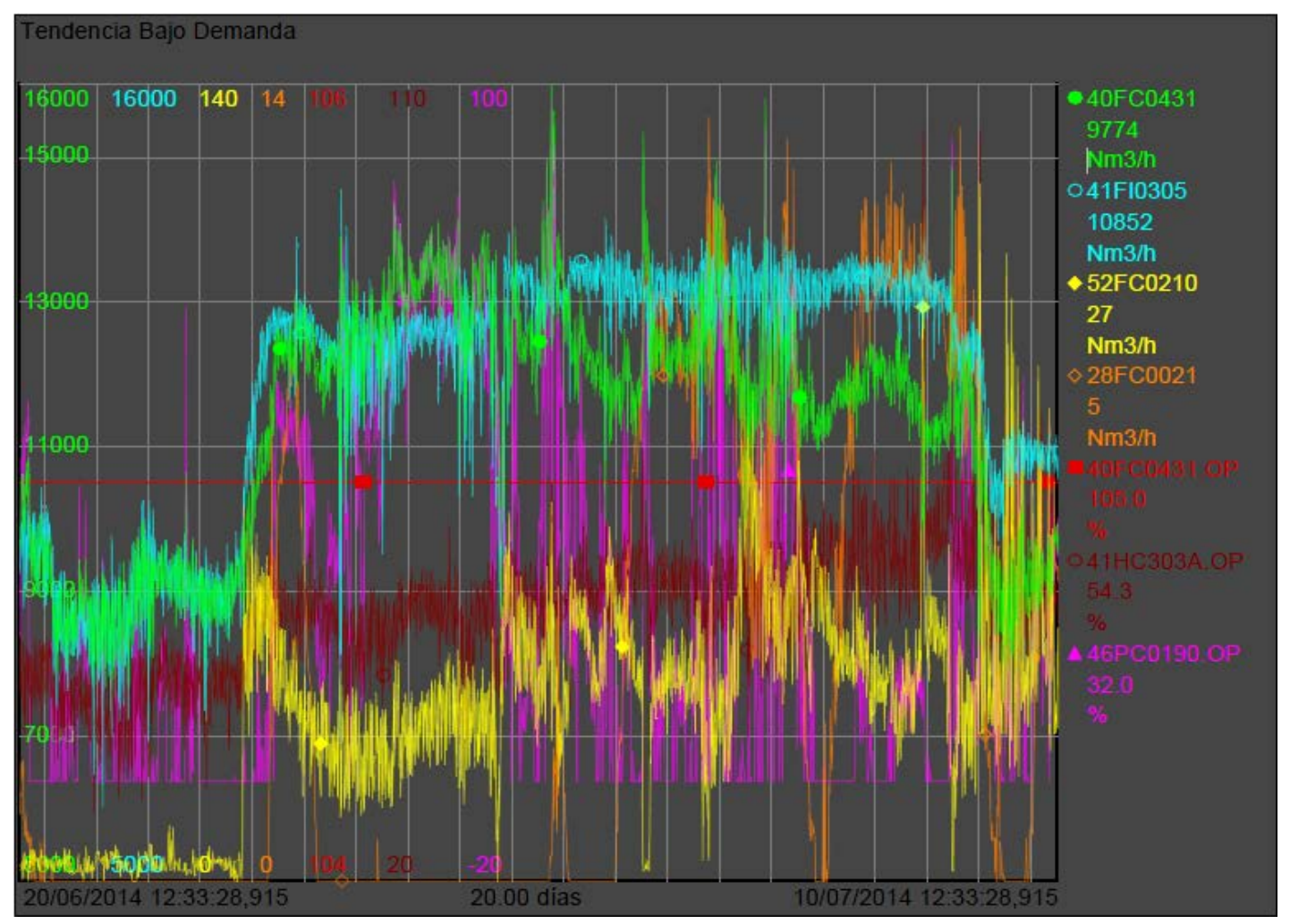

Figure 5.2. Example of drift error probably due to operation close to the upper range of span. 


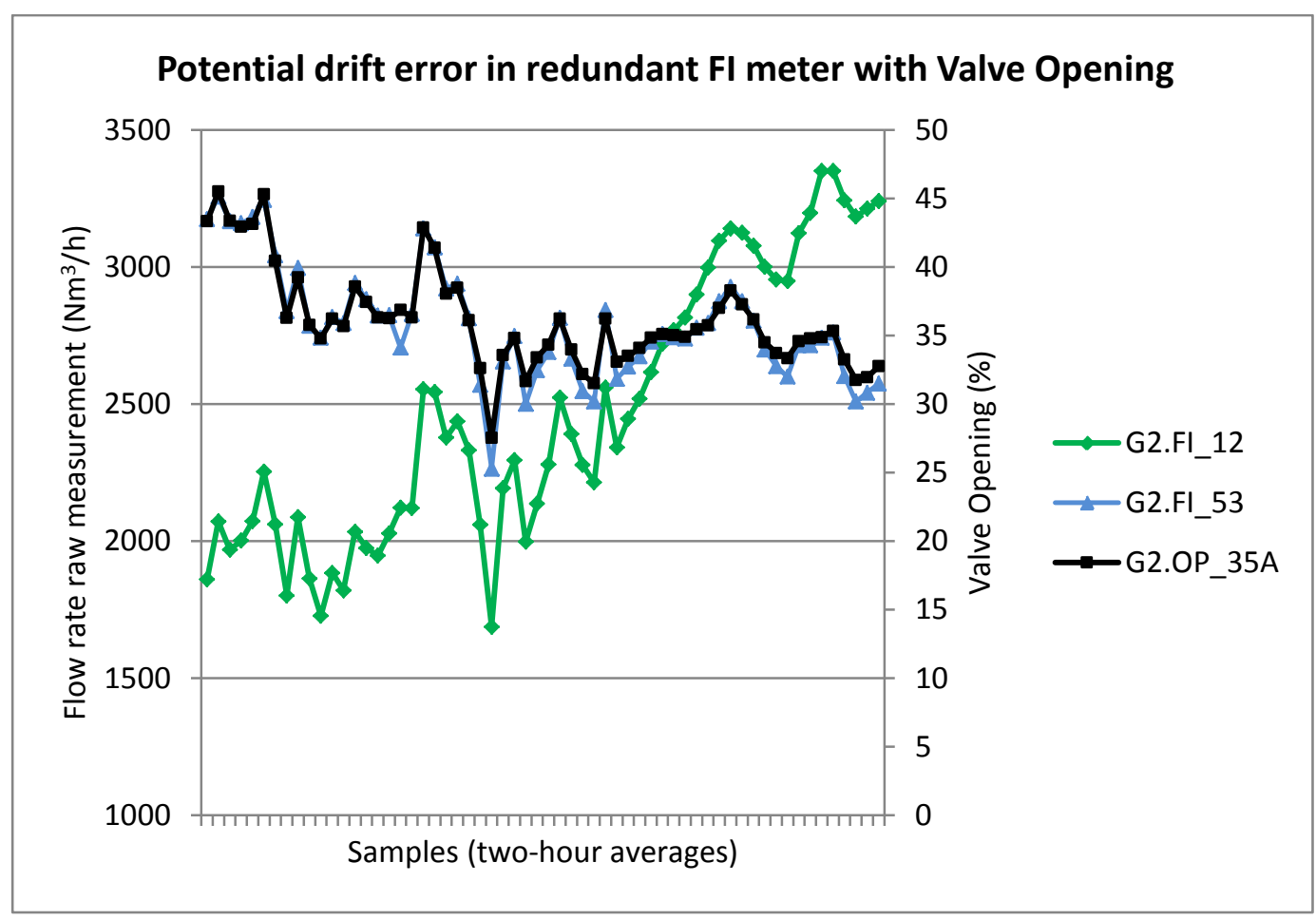

Figure 5.3. Example of drift error in two redundant flowmeters, compared with available valve opening.

The main sources of uncertainty regarding flow measurement with an orifice-plate meter are: the discharge coefficient, the differential pressure measurement, the fluid density, the compressibility factor, and the internal diameters of the plate and the pipe. These are briefly explained next, based on available references as ISA-Spain and Daniel Measurement Inc.

The coefficient of discharge of an orifice is determined empirically so that the particular orifice meter being installed must reproduce as closely as possible the installation on which the tests were run whether they are specific tests on the unit itself or general tests run by the various standards agencies. The flow pattern across the plate is very important for flow measurement accuracy. There are two factors which control this pattern: piping configuration, including length, roundness, smoothness, and nearest fitting such as elbows, valves, tees; and the second is the Reynolds Number, which is the guide to the shape, size and stability of the vena contracta. Most gas is handled at relatively high Reynolds Numbers in the range where the coefficient becomes stabilized, which helps.

The accurate calculation of flow through an orifice requires a correct differential pressure and a correct density of the flowing gas and proper interpretation of their effects on the performance on the meter. One of the major sources of error in the application of an orifice is the problem of taking the square root of the differential measurement of pressure and the effects of small errors in the differential at low differentials. Good practice then dictates that the differential must be kept as high as possible within the limitations of the range of the flow fluctuation.

It is of equal importance that the orifice plate be handled with care and a regular inspection routine should be instigated to insure the maximum quality of the condition of the plate at all 
times. While the orifice plate is the most inexpensive item, it is the most important part, as its physical condition can be largely responsible for the overall accuracy of the measurement. The orifice plate must be properly centered and housed. Another source of error is the effect of time on the orifice and the meter tube. No known pipeline is completely clean. The best that can be expected is a minimum of rust, oil vapors, condensed liquids, lubricating greases and the like. Any of these deposited on the plate and tube in the right places can cause errors of 5 to $10 \%$ easily. Therefore, the plate and the tube should be periodically inspected, cleaned and rechecked.

\subsection{2- Data Reconciliation Formulation}

The data reconciliation problem is formulated as the solution of the minimization of the sum of square errors of the deviations model-measurements, according to the objective function $(J)$ and subject to model equality constraints $(f)$ and other additional non-equality constraints such as bounds $(g)$,

$$
\begin{gathered}
\min _{u, \theta, \varepsilon} J=\sum_{i=1}^{N} f_{i}^{\text {reliab }} \cdot\left(\frac{F_{i}^{\text {measured }} \cdot \beta_{i}^{\text {comp }}-F_{i}^{\text {model } l}}{\operatorname{span}_{i}}\right)^{2}+f^{\text {weig }} \cdot \sum_{j=1}^{M} f_{j}^{\text {reliab }} \cdot \varepsilon_{j}^{2} \\
\text { s.t. } \quad \begin{array}{l}
\boldsymbol{f}(\boldsymbol{x}, \boldsymbol{u}, \boldsymbol{\theta}) \\
\boldsymbol{g}(\boldsymbol{x}, \boldsymbol{u}, \boldsymbol{\theta}) \leq 0
\end{array}
\end{gathered}
$$

where $\boldsymbol{x}$ is the vector of process states, $\boldsymbol{u}$ the input or manipulated variables, and $\boldsymbol{\theta}$ the model parameters. The objective function comprises model-measurement deviations for all available flowmeters $N$; the treatment for all on-line analysers $M$ differs and is explained below. An NLP problem results; all terms and considerations involved are expounded on next.

\section{Compensation for raw measurements}

The reference for model flow rates $F_{i}^{\text {model }}$ is not the raw measurement $F_{i}^{\text {measured }}$ but a compensated one $F_{i}^{\text {compensated }}$, where the compensation factor $\beta_{i}^{\text {comp }}$ allows to correct for process deviations in operating conditions $(o p)$ from fixed calibration/design conditions (des) in pressure $(P)$, temperature $(T)$ and molecular weight $(w)$ of gas stream. The compensation factor is derived from mass and energy balances applied to the computation of the flow rate for the orifice-plate flowmeter with a differential pressure transmiter, see for example Acedo Sánchez (2006) or ISA-Spain manuals.

$$
\begin{aligned}
& F_{i}^{\text {compensated }}=F_{i}^{\text {measured }} \cdot \beta_{i}^{\text {comp }} \\
& \beta_{i}^{\text {comp }}=\sqrt{\frac{T_{\text {dis }} \cdot w_{\text {dis }}}{P_{\text {dis }}}} \cdot \sqrt{\frac{P_{o p}}{T_{o p} \cdot w_{o p}}}
\end{aligned}
$$




$$
\beta_{i}^{\text {comp }}=\max \left(\min \left(\sqrt{\frac{T_{i}^{\text {des }} \cdot w_{i}^{\text {des }}}{P_{i}^{\text {des }}}} \cdot \sqrt{\frac{P_{i}^{o p}}{T_{i}^{o p} \cdot w_{i}^{o p}}}, 1.20\right), 0.80\right)
$$

The correction factor $\beta_{i}^{\text {comp }}$ has been arbitrarily truncated to the range $[0.80,1.20]$ according to practical experience and knowledge about valid range for correction: the compensated measurement is not reliable in cases of high $\beta_{i}^{\text {comp }}$.

\section{Analyser treatment}

Deviations for analysers Al are not weighted as errors to minimize in the objective function $J$, but included as non-linear hard constraints to fulfil. Slack variables $\varepsilon_{j}$ are additional degrees of freedom, heavily weighted with $f^{\text {weig }}$ as compared to deviations in flow rates in order to force a negligible value for $\varepsilon_{j}$ in the optimum achieved, i.e. that the deviation $y_{j}^{\text {measured }}-y_{j}^{\text {model }}$ is subject to a certain tolerance $\left[-y_{j}^{\text {tol }}, y_{j}^{\text {tol }}\right]$. Slack variables help guide the advance towards the solution while at the same time guaranting numerical feasibility in any case.

$$
\begin{aligned}
& -y_{j}{ }^{\text {tol }} \leq y_{j}^{\text {measured }}-y_{j}{ }^{\text {model }}+\varepsilon_{j} \leq y_{j}{ }^{\text {tol }} \\
& -10 \leq \varepsilon_{j} \leq 10
\end{aligned}
$$

There are several reasons why deviations for analysers have not been included in the objective function, in contrast to deviations for flowmeters:

- analysers are much more reliable in general as compared to flowmeters, and do not suffer from uncertainty due to operating conditions different from the design ones;

- its number is scarce, without redundancy, usually only one per consumer plant (in the HP system) and only for important consumer plants, not for smaller ones. In fact, only in three consumer plants (G4, HD3, NC6) redundancies occur regarding $\mathrm{H}_{2}$ purity measurements, an analyser being present both in the HP separator gas outlet and in the total gas to reactor stream sum of make-up and recycle streams, and experience shows that usually these two analysers do not provide addional useful information but merely follow each other, maintaining the same systematic error in different scenarios;

- the $\mathrm{H}_{2}$ purity in the HP system is very sensitive to reactor $\mathrm{H}_{2}$ consumption and make-up terms, thus a narrow margin has to be allowed to take advantage of the analyser measurements, whereas soft-constraints or penalty terms in the objective function do not guarantee strict fulfilment;

- the different model sensitivity to flow rates and the HP system $\mathrm{H}_{2}$ purity, along with the difficulty to balance non-homogeneous terms in the objective function. More consistent results regarding flowmeters are achieved due to this approach, because the chance of local optima is decreased. 
Tolerance for deviations in analysers $y_{j}^{\text {tol }}$ has been fixed according to measurements for redundant analysers, historical laboratory data, and trial and error reconciliation experiments performed, taking into account that lower tolerances mean greater error for flowmeter fitting and vice versa. Its value is specified in $0.020 \% 1 \mathrm{~mol}$ for analysers based on gas chromatography and higher in $0.030 \% 1 \mathrm{~mol}$ for those based on density measurements, because of the uncertainty when purity is inferred from density due to variability in the light ends composition.

Another rational approach would have been to fix as known inputs the necessary and suficcient number of analyser measurements; however as a consequence linearity would have been lost in the constraints arising from the subsytem comprising all the mass balance equations for gas streams together with the consumption/generation terms in reactors. Linearity is a desirable feature to bind the search region in order to guarantee model simulation convergence, therefore it has been preferred to maintain the non-linear constraints for $\mathrm{H}_{2}$ purity analysers. Furthermore, it would have been difficult to assign tolerances when fixing a particular value for the Al measurement.

\section{Feasibility}

Deriving from a closed subsystem of mass balance equations, constraints for model flow rates $F_{i}$ are linear, thus easy to handle. On the contrary, constraints for analysers are non-linear and the allowed range is narrow as aforementioned. Therefore, slack variables $\varepsilon_{j}$ have been introduced for each analyser to guarantee numerical feasibility and to help guide the advance towards the solution. Slack variables have not been used for flow rates $F_{i}$ constraints; even for degraded functioning of the instruments, allowed limits have enabled a feasible solution in experiments along different years. Only for occasional important failures in flowmeters infeasibility in linear constraints happened, easily detected by means of the Lagrange multiplier and corrected by assigning zero weight $f_{i}^{\text {reliab }}$ to the faulty instrument, as a result disregarded for reconciliation and unlimited.

\section{Normalization}

Normalization of model-measurement errors for flowmeters is performed with the instrument span $\left(\operatorname{span}_{i}\right)$. For purely random errors, on-line standard deviation of measurement $\sigma_{i}$ is used to normalize because this leads to the maximum-likelihood estimate, LITER. Nevertheless, for this application where consistency and robustness are important issues due to potential unknown drift errors combined with multiple local optima, a weighting factor changing on-line could cause troubles related to transient states, where standard deviations are higher typically 2-4 times those for stationary operation, thus changing the relative priorities of flowmeters. It has been considered however that instruments relative accuracy remains the same in the transient states. Furthermore, redundancy which is the basis for data reconciliation usually appears in local nodes with small capacity for storage, and where conservation laws not accounting for dynamic effects still fulfil in spite of the transients states. Therefore, the on-line change in normalization according to $\sigma_{i}$, although not extremely determining for the solution, could introduce non-desired additional inconsistency in particular cases and was disregarded. 


\section{Scaling}

In order to guarantee proper SNOPT ${ }^{\circledR}$ performance, all decision and constraint variables have been scaled to the order of magnitude of units depending on their initial values, thus avoiding an ill-conditioned Jacobian matrix, whereas the objective function has been scaled to an appropriate order of magnitude to assure an adequate sensitivity. The scaling of decision and constraint variables depending on their initial values has proved very determining for reaching a good solution. Although SNOPT ${ }^{\circledR}$ support different functions for automatic scaling, better results were obtained with the aforementioned one.

\section{Robust estimators}

Robust estimators as the Fair or the Welsch functions have not been used. All measurements affected by gross errors have been previously identified and removed, thus avoiding their undesirable effects on the reconciled results like biased estimations and the spread of errors among the whole network. Besides, customised ranges have been allowed for all measured variables, thus binding the search region conveniently avoiding excessive deviations, and making the use of the WLS estimator non detrimental. Moreover, no additional tuning parameters arise with the WLS approach, unlike what happens with robust estimators.

\section{Average values}

Two-hour average values were used for the data reconciliation; smaller periods could be misleading because of process variability and interactions among controllers for flow rate and pressure.

\section{Tuning parameters}

The global and individual parameters to be tuned are listed below, with some general guidelines.

Global tuning parameters:

- Scaling factor for the objective function. Good convergence has proved very sensitive to this scaling factor, and although a default one usually worked correctly, occasionally a change in the order of magnitude was necessary, probably because of the EcosimPro ${ }^{\circledR}$ drawback of not incorporating automatic differentiation.

- Weight $f^{\text {weig }}$ for analyser slack variables has been tuned according to the objective function scaling factor, allowed tolerance for analyser constraints $y_{j}^{\text {tol }}$, and the desired range for $\varepsilon_{j}$ at optimum. A fixed value is valid for all experiments.

Individual tuning parameters:

- Percentage over measurement $f_{i}^{\text {Uplo }}$ to compute upper/lower bounds for flowmeters. One individual value for each meter. It should be noticed that upper and lower bounds 
for flow rates $F_{i}$ depend on the measured value, not on the instrument span, thus not being constant.

$$
F_{i}^{\text {measured }} \cdot\left(1-f_{i}^{\text {UpLo }}\right) \leq F_{i} \leq F_{i}^{\text {measured }} \cdot\left(1+f_{i}^{\text {UpLo }}\right)
$$

The $f_{i}^{\text {UpLo }}$ cannot be set to a very low value even in the absence of redundancy, because it is centred on the raw measurement and has to allow for the compensation factor $\beta_{i}^{\text {comp; }}$ that is for example the case of recycle streams without redundant measurements. Its value is also related with expected imbalance in redundant nodes, in order to guarantee feasibility for changing operating conditions. The guideline has been to set the lowest value enabling feasibility and the needed margin for $\beta_{i}^{\text {comp }}$, because higher values (broader search regions) do not lead to more accurate and consistent results, quite the contrary due to increased chance of local optima. In general, in headers linked to producer plants $f_{i}^{\text {Uplo }}$ has been set to around 0.05-0.10 depending on the individual flow meter $\beta_{i}^{\text {comp }}$, whereas in the LPH and HP purges $f_{i}^{\text {UpLo }}$ has been set to around $0.15-0.25$ because of higher $\beta_{i}^{\text {comp }}$, higher variability in molecular weight and higher imbalances in redundant nodes. Maximum values of up to 0.65 for flowmeters in the MP/LP system have been used, due to higher uncertainty and process variability. A good tuning of all $f_{i}^{\text {Uplo }}$ factors is determining.

- Allowed tolerance $\left[-y_{j}^{\text {tol }}, y_{j}^{\text {tol }}\right]$ for analyser measurements. Although one individual tolerance can be specified for every meter, common values of \pm 0.020 and $\pm 0.035 \% 1$ mol have been fixed according to the analyser technology.

- The factors $f_{i}^{\text {reliab }}, f_{j}^{\text {reliab }}$ accounting for instruments $\mathrm{Fl}, \mathrm{Al}$ reliability. According to programmed data treatment rules, the factor can automatically be set to zero for both flowmeters and analysers. In addition, the user can optionally manually assign a value in the range $[0,1]$ to allow for different reliability.

\subsection{3- Data Reconciliation Degrees of Freedom and Constraints}

Model $f(\boldsymbol{x}, \boldsymbol{u}, \boldsymbol{\theta})=0$ comprises equations stated in Chapter. Degrees of freedom $(\boldsymbol{u}, \boldsymbol{\theta})$ for the data reconciliation problem are listed in Table 5.1; they are equal to those of the model, excluding variables characterizing hydrocarbon liquid streams. In the selection of boundaries for gas flow rates, priority has been given to measured streams, and in case of multiple choices, to the prospective most accurate one. Streams with valves usually closed, typically those corresponding to reliefs to fuel gas headers under pressure control activated as second branches in split-range control structures, are also preferred as boundaries.

Constraints are included in Table 5.2. The non-linear constraint due to the upper and lower bounds for the ratio of light ends generated in the reactor to the $\mathrm{H}_{2}$ consumed is transformed into two linear constraints, with an arbitrarily fixed 1000 upper limit. 


\begin{tabular}{|c|c|c|c|}
\hline Variable & Description & Units & Number \\
\hline$F$ & $\begin{array}{l}\text { Volumetric flow rate Normal conditions } \\
\text { gas stream (either directly or } \\
\text { through ForceFbnd in valves) }\end{array}$ & $\mathrm{Nm}^{3} / \mathrm{h}$ & 120 \\
\hline$\Delta R_{H 2}^{s p}$ & Specific $\mathrm{H}_{2}$ consumption & $\mathrm{Nm}^{3} \mathrm{H}_{2} / \mathrm{m}^{3} \mathrm{HC}$ & 16 \\
\hline$\Delta R_{L I G}{ }^{s p}$ & Specific light ends generation in volume & $\mathrm{Nm}^{3} \mathrm{LIG} / \mathrm{m}^{3} \mathrm{HC}$ & 16 \\
\hline$\Delta R_{w L / G}$ & Molecular weight of light ends generated & kg LIG/kmol LIG & 16 \\
\hline$k_{\text {gasHC }}^{S}$ & Gas solubility in hydrocarbon & $\mathrm{Nm}^{3} / \mathrm{m}^{3} \mathrm{HC}$ & 27 \\
\hline$k_{\alpha H 2 L I G}^{S^{2}}$ & $\begin{array}{l}\text { Relative solubility for } \mathrm{H}_{2} \text { and light ends in } \\
\text { gas and liquid streams }\end{array}$ & - & 27 \\
\hline$k_{W L I G}^{S}$ & $\begin{array}{l}\text { Relative solubility for light ends in gas } \\
\text { and liquid streams, that is, } \\
\text { distribution coefficient for light ends } \\
\text { molecular weight }\end{array}$ & - & 27 \\
\hline$\varepsilon$ & $\begin{array}{l}\text { Slack variable for non-linear constraints } \\
\text { regarding analysers }\end{array}$ & $\% 1 \mathrm{~mol}$ & 14 \\
\hline
\end{tabular}

Table 5.1. Decision variables for the data reconciliation problem.

\begin{tabular}{|c|c|c|c|}
\hline Constraint & Description & Units & Number \\
\hline Analyser & $-y_{j}^{\text {tol }} \leq y_{i}^{D T}-y_{i}+\varepsilon_{1} \leq y_{j}^{\text {tol }}$ & $\% 1 \mathrm{~mol}$ & 14 \\
\hline \multirow{3}{*}{$\begin{array}{l}\text { Ratio Light ends } \\
\text { generated }-\mathrm{H}_{2} \\
\text { consumed }\end{array}$} & $0 \leq \Delta R_{L I G}{ }^{s p}-\Delta R_{L I G H 2}{ }^{\text {LoLim }} * \Delta R_{H 2}{ }^{s p} \leq 1000$ & $\mathrm{Nm}^{3} \mathrm{LIG} / \mathrm{m}^{3} \mathrm{HC}$ & 16 \\
\hline & $0 \leq \Delta R_{\text {LIGH }}{ }^{\text {UpLim }} * \Delta R_{H 2}{ }^{s p}-\Delta R_{\text {LIG }}{ }^{s p} \leq 1000$ & $\mathrm{Nm}^{3} \mathrm{LIG} / \mathrm{m}^{3} \mathrm{HC}$ & 16 \\
\hline & $\begin{array}{l}\text { (for platformer plants, lower and upper } \\
\text { bounds are }-1000 \text { and } 0 \text { instead of the } \\
\text { values for consumer plants } 0 \text { and } 1000 \text { ) }\end{array}$ & & \\
\hline $\begin{array}{l}\text { Explicit gas flow } \\
\text { rates }\end{array}$ & see Table 5.5 & $\mathrm{Nm}^{3} / \mathrm{h}$ & 142 \\
\hline
\end{tabular}

Table 5.2. Constraints for the data reconciliation problem. 
Problem dimension is shown in Table 5.3. The number of model equations is 14136 , and the Jacobian size 103.103 due to algebraic variables linked to non-linear boxes.

\begin{tabular}{lcc}
\hline Optimization Problems & Data Reconcil. & Opt. Redistrib. \\
\hline № Independent Degrees of Freedom & 263 & 47 \\
№ Total Independent Constraints & 188 & 203 \\
no Independent NL-Constraints & 14 & 25 \\
\hline
\end{tabular}

Table 5.3. Dimension for optimization problems: $\mathrm{n}$ ㅇ independent dof/constraints.

Constraints for explicitly calculated gas flow rates are added to assure a positive value. No constraints are added for streams modelled with "h2dir" ports, as their purpose it to model both positive and negative flow rates, unless either a flowmeter or a valve are available. Besides, and again unless either a flowmeter or a valve are available, the following constraints to assure a positive flow rate are removed because they are redundant necessarily with those corresponding to other boundary or explicit streams:

- Inlet flow rate to valves "dtOh2", as it is equal to the variable specified as bound, the so called "ForceFbnd";

- Inlet flow rate to splitter components, as it is redundant with the two outlet streams having positive flow rates. The same for the inlet to v3v_2out valves;

- Outlet flow rate of mixer components, as it is redundant with the two inlet streams having positive flow rates. The same for the outlet of $v 3 v \_2$ in valves;

- For valve components $v 3 v \_2 i n$ and $v 3 v \_2 o u t$, flow rates for the two corresponding mutually exclusive streams, whose value depending on the valve position is either zero or either equal to that of another stream already limited as boundary or explicit, respectively the outlet for $v 3 v \_2$ in and the inlet for $v 3 v \_2 o u t$;

- All gas flow rates for mixtures of gas and liquid hydrocarbon, being the gas either totally solved or constituting a two-phase mixture, as it is redundant

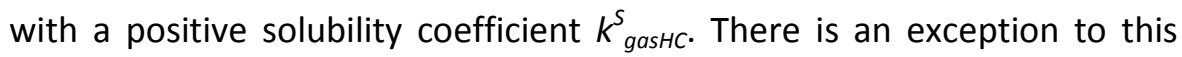
rule, a constraint is added to assure positive gas flow rate in mixtures gashydrocarbon for reactor outlets which are mixed with a gas stream (acting as a reactor bypass) before the HP separator; otherwise separator gas outlet can be positive without implying a positive reactor gas outlet. It is manually configured, unlike all the previous configured variables and 
limits, because the effort to do it automatically is not worthy being a step in model configuration, therefore only made once.

\section{Limits and Initialization}

Limits and initialization values are extremely important to solve the optimization problem. Parameters for reactors $\left(\Delta R_{H 2}{ }^{s p}, \Delta R_{L I G}{ }^{s p}, \Delta R_{w L I G}, \Delta R_{L I G H 2}\right)$ and separators $\left(k_{\text {gasHC }}^{S}, k_{\text {aH2LIG }}^{s}, k_{\text {wLIG }}^{s}\right)$ have fixed lower/upper bounds, equal for any experiment (see Table 5.4); also lower/upper bounds for slack variables $(\varepsilon)$ are fixed. A rational approach is obeyed to fix bounds on degrees of freedom:

- Broad range for specific consumption $\Delta R_{H 2}{ }^{s p}$, in order to get an estimation based on measurements without any binding due to a fixed range;

- Although a broad range is specified for the degree of freedom specific generation $\Delta R_{L I G}{ }^{s p}$ of consumer plants, it is indirectly bound in a narrow range by means of the ratio $\Delta R_{L I G H 2}$ according to process knowledge. On the contrary, a broad range is specified in the case of platformers, because of the higher magnitude and variability, being finely tuned thanks to the platformers purity analysers;

- Narrow range for $\Delta R_{w L I G}$, according to process knowledge and also considering the poor identifiability in the overall fitting of this parameter. For certain consumer plants it has additionally a low sensitivity, whereas for others as the catalytic-reforming producer plants and the biggest consumer plants, the sensitivity is more significant;

- Broad range for $k_{\text {gasHC, }}^{S}$ as flow measurements in gas outlets of MP/LP separators exist for an accurate estimation;

- Medium-narrow range for $k_{\alpha H 2 L G \text {, }}^{S}$ in order to allow for certain process variability while guaranteeing process consistency according to operating conditions and not too much flexibility to the optimization routine;

- Narrow range for $k_{w L I G}^{S}$ also in order to allow for certain process variability while guaranteeing process consistency according to operating conditions and not too much flexibility to the optimization routine. This parameter does not present a significant sensitivity, thus being less important;

- Broad range for $\varepsilon$ in between $[-10,10]$ in order to ensure feasibility, and taking into account that a negligible value at optimum will be achieved due to its very large weight in the objective function to minimize. 


$\begin{array}{llc}\Delta R_{H 2}{ }^{s p} & \text { broad range, not limiting } & \mathrm{Nm}^{3} \mathrm{H}_{2} / \mathrm{m}^{3} \mathrm{HC} \\ \Delta R_{L I G}{ }^{s p} & \text { broad range, not limiting } & \mathrm{Nm}^{3} \mathrm{LIG} / \mathrm{m}^{3} \mathrm{HC} \\ \Delta R_{w L I G} & \sim \pm 10 \% & \mathrm{~kg} \mathrm{LIG} / \mathrm{kmol} \mathrm{LIG} \\ \Delta R_{L I G H 2} & \sim \pm 40 \% & \mathrm{Nm}^{3} \mathrm{LIG} / \mathrm{Nm}^{3} \mathrm{H}_{2} \\ & & \\ k_{\text {gasHC }}^{S} & \text { broad range, not limiting } & \mathrm{Nm}^{3} / \mathrm{m}^{3} \mathrm{HC} \\ k^{S}{ }_{\alpha H 2 L I G} & \sim \pm 15 \%(\mathrm{HP}) \sim \pm 20 \%(\mathrm{MP}) & - \\ k^{S}{ }_{\text {wLIG }} & \sim \pm 10 \%(\mathrm{HP}) \sim \pm 10 \%(\mathrm{MP}) & -\end{array}$

Table 5.4. Allowed range for model parameters, percentage over default value.

Variable

Lower/Upper Bounds

Measured gas flow

IF $\left(F_{i}^{\text {weigh }} \neq 0\right)$ AND $\left(F_{i}^{\text {measured }}>F^{\text {threshold }}\right)$ :

rate $\left(F_{i}\right)$, "h2" port $F_{i}^{\text {measured }} \cdot\left(1-f_{i}^{\text {UpLo }}\right) \leq F_{i} \leq F_{i}^{\text {measured }} \cdot\left(1+f_{i}^{\text {Uplo }}\right)$

$\operatorname{ELSEIF}\left(F_{i}^{\text {weigh }} \neq 0\right) \operatorname{AND}\left(F_{i}^{\text {measured }}<F^{\text {threshold }}\right)$ :

$0 \leq F_{i} \leq \max \left(25, F_{i}^{\text {measured }} \cdot\left(1+f_{i}^{\text {Uplo }}\right)\right)$

ELSEIF $F_{i}^{\text {weigh }}=0$ :

$0 \leq F_{i} \leq F_{\infty}$

Measured gas flow

rate $\left(F_{i}\right)$, "h2dir" port

IF $\left(F_{i}^{\text {weigh }} \neq 0\right)$ AND $\left(F_{i}^{\text {measured }}>F^{\text {threshold }}\right)$ :

$F_{i}^{\text {measured }} \cdot\left(1-f_{i}^{\text {Uplo }}\right) \leq F_{i} \leq F_{i}^{\text {measured }} \cdot\left(1+f_{i}^{\text {Uplo }}\right)$

$\operatorname{ELSEIF}\left(F_{i}^{\text {weigh }} \neq 0\right)$ AND $\left(F_{i}^{\text {measured }}>0\right)$ :

$-F^{\text {threshold }} \leq F_{i} \leq \max \left(25, F_{i}^{\text {measured }} \cdot\left(1+f_{i}^{\text {UpLo }}\right)\right)$

$\operatorname{ELSEIF}\left(F_{i}^{\text {weigh }} \neq 0\right)$ :

$-F_{\infty} \leq F_{i} \leq F^{\text {threshold }}$

$\operatorname{ELSEIF}\left(F_{i}^{\text {weigh }}=0\right)$ :

$-F_{\infty} \leq F_{i} \leq F_{\infty}$

Non-measured gas

flow rate $\left(F_{i}\right)$ with

IF $\left(F_{i}^{\text {OPvalve }}<F_{i}^{\text {OPthreshold }}\right)$ :

$0 \leq F_{i} \leq 25$

valve opening, "h2"

ELSEIF $\left(F_{i}^{\text {OPvalve }}>F_{i}^{\text {OPthreshold }}\right)$ :

$0 \leq F_{i} \leq F_{\infty}$

Non-measured gas $\quad-F_{\infty} \leq F_{i} \leq F_{\infty}$

flow rate $\left(F_{i}\right)$, "h2dir"

port (no valve

openings available in

this case)

Table 5.5. Limits for gas flow rates, both Bound and Explicit. 
However, both lower/upper bounds and initialization values for gas flow rates are different depending on the experiment; the treatment is analogous for all gas flow rates, irrespective of their category as either boundaries or explicit variables (see Table 5.5). An initial value for boundary gas flow rates is assigned equal to the measured value, whereas lower/upper limits are assigned centred on the said measured value.

$F^{\text {threshold }}$ is obtained as a $10 \%$ of the span, enabling to deal with bias when a flow meter is measuring close to zero flow rate. It has been observed that in certain cases, the $\mathrm{Fl}$ reading can be a certain amount due to a bias error, whereas redundant measurements and closed valves indicate zero flow rate. A custom range $f_{i}^{\text {Uplo }}$ is specified for each stream $F_{i}$ as a function of the flow rate raw measurement, according to expected compensation and potential imbalance of involved measurements.

Initialization rules for flow rates, either bound or explicit, are generated automatically, whereas initialization rules for valves and other excluding streams, particularly in headers, are generated with ad hoc code. In order to assign either a zero flow rate or a certain flow direction, this ad hoc code comprises different nested conditional branches combining and comparing rules like:

- $F_{i}^{\text {measured }} \geq F_{k}^{\text {measured }} \quad$ two flow measurements compared

- $F_{i}^{\text {measured }} \geq F^{\text {threshold }} \quad$ flow measurement compared with threshold

- $F_{i}^{\text {OPvalve }} \geq F_{k}^{\text {OPvalve }} \quad$ two valve openings compared

- $F_{i}^{\text {OPvalve }} \geq F_{k}^{\text {OPthreshold }} \quad$ valve opening compared with threshold (typically 0 )

- $\quad\left(T_{1}\right.$ reactor $\left.{ }^{\text {measured }} \geq T^{\text {threshold }}\right)$ AND $\left(T_{2 \_ \text {reactor }}^{\text {measured }} \geq T^{\text {threshold }}\right)$ plant in ON state, according to 2 reactor temperatures

- $\quad\left(T_{1}\right.$ reactor $\left.{ }^{\text {measured }} \leq T^{\text {threshold }}\right)$ AND $\left(T_{2_{-} \text {reactor }}^{\text {measured }} \leq T^{\text {threshold }}\right)$ plant in OFF state, according to 2 reactor temperatures

Whenever possible, rules comparing flow measurements are preferred to assign a particular flow direction. In order to force a zero flow rate, a valve opening is preferred. In general, in headers and pipes allowing either zero flow rate or one flow direction or the opposite, more than one nested conditional branch is needed to initialize the corresponding flow rate variable, comparing different types of measurements combined with AND/OR logical expressions.

No additional limits have been specified for output variables regarding compositions, neither for $\mathrm{H}_{2}$ purities nor for molecular weights. The approach preferred is to calibrate solubility model parameters and fixed a narrow range, which results in bound purities and compositions for all streams. In this way, the underlying model provides physical consistency and compositions in the MP and LP separators follow the trends in the HP separator: the higher the purity in the HP separator gas outlet, the higher the purity in the MP separator gas outlet, and so on.

As aforementioned, appropriate limits both for decision and constraints variables are extremely important; too broad limits can lead to suboptimal solutions because the probability of local optima increases, whereas too narrow ones (even for a particular variable) can lead also to bad results due to lack of flexibility in other related variables along the steps of the search. The particular trade-off depends on the variable and the problem solved. 


\section{5- DATA RECONCILIATION IMPLEMENTATION}

Implementation has been performed in EcosimPro $^{\circledR}$, with a SNOPT ${ }^{\circledR}$ SQP algorithm as optimization solver, following a direct sequential approach (Figure 5.4). The SQP routine was chosen according to the characteristics of the problem being solved, non-linear and with a medium number of decision variables and constraints; being the solver deterministic, a solution is obtained in a reasonable computation time. Another implementation is also available in $\mathrm{GAMS}^{\circledR}$ modelling and optimization environment (G. Gutiérrez, D. Sarabia et al.), following a simultaneous approach and using the same SNOPT ${ }^{\circledR}$ SQP solver. Whereas in EcosimPro ${ }^{\circledR}$ execution times for the data reconciliation problem range from 5 hours to 50 hours in an Intel ${ }^{\circledR}$ Core $^{\mathrm{TM}} \mathrm{i} 7,2.80 \mathrm{GHz}, 4.00 \mathrm{~GB}$ RAM, being the execution time to run a steady state of 1 second approximately, in GAMS ${ }^{\circledR}$ execution times are in the order of 2 to 10 minutes, with about 5 additional minutes for importing results in the developed Excel ${ }^{\circledR}$ user interface. One of the main drawbacks of EcosimPro ${ }^{\circledR}$, not only due to the higher required time to perform simulations for computing gradients but also due to the decreased accuracy of these gradients computed by finite differences, is the lack of automatic differentiation methods for the computation of derivatives.

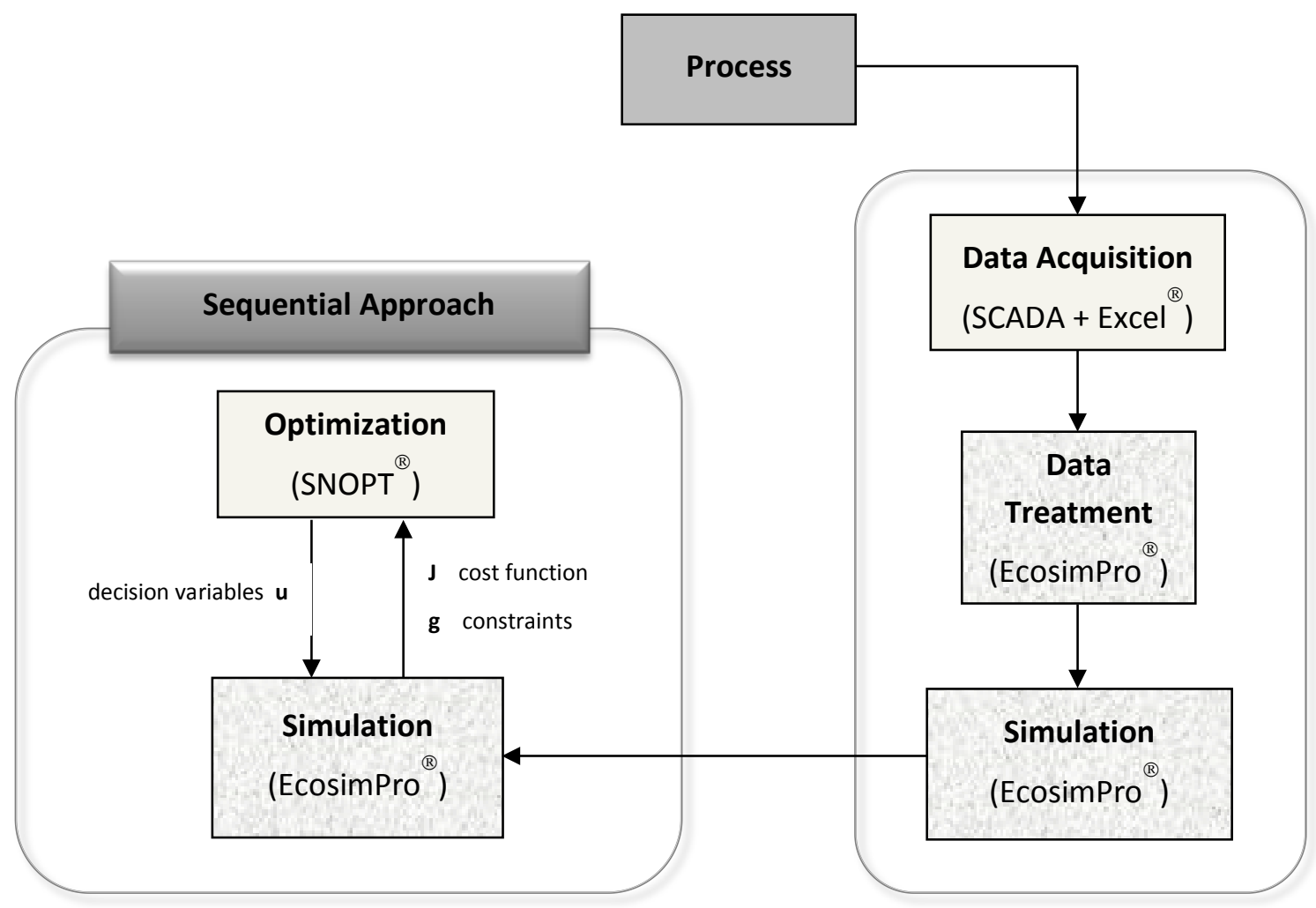

Figure 5.4. Scheme for the data reconciliation implementation in EcosimPro ${ }^{\circledR}$. 
Both the data acquisition and the optimization are automatically executed, but need to be manually triggered. All results for the different scenarios have been obtained with the same configured parameters, except for the scale factors affecting the objective function and the slack variables for analysers, using the same value for all slack variables; for a few data sets it was necessary to change the default ones in order to obtain good results because otherwise the optimization algorithm finished without fulfilling purity constraints for analysers. Experience has shown that an adequate tuning of scale factors determines to a great extent the solution achieved with the SNOPT ${ }^{\circledR}$ algorithm, may be in this case where EcosimPro ${ }^{\circledR}$ does not provide automatic derivatives for model variables.

\subsection{1- Linear constraints management}

Two subsequent calls to SNOPT ${ }^{\circledR}$ are made, because of SNOPT ${ }^{\circledR}$ treatment of linear constraints in the algorithm employed. According to the SNOPT ${ }^{\circledR}$ manual, and also checked experimentally, bounds specified for constraints identified as linear $h$ apply to the linear terms excluding the independent term, i.e., bounds are applied to: $I_{10} \leq A x \leq I_{u p}$, instead of being applied to: $I_{10} \leq A x+B \leq I_{\text {up }}$, where $x$ is the vector of decision variables, $I_{10 / \text { up }}$ the lower/upper bounds for the linear constraints, $A$ the matrix of fixed coefficients, and $B$ the vector of independent terms. Nevertheless, it is bounds for the complete constraints $h$ what is known, and as a consequence the applied bounds $I_{\text {Io/up }}{ }^{\text {applied }}$ must be computed by subtracting the independent term $B$ from the natural bounds $I_{\text {lo/up }}{ }^{\text {natural }}$. Linear constraints usually correspond to flow rate variables.

In order to handle this SNOPT ${ }^{\circledR}$ treatment of linear constraints, and taking advantage of two different functions available in the SNOPT ${ }^{\circledR}$ commercial library, the dynamic-link library (dII) developed at the Dpt. (J.A. Caminero and C. Gómez Palacín) to be used from EcosimPro ${ }^{\circledR}$ incorporates functionality to perform the optimization in a two-stage procedure, see Figure 5.5. These are the successive steps:

1. First, a SNOPT ${ }^{\circledR}$ function computing the optimization problem structure is called. This function also identifies which constraints are linear, and returns the matrix $A$ of fixed coefficients for linear constraints as a function of decision variables. In order to avoid simulation convergence problems due to out of range combinations of decision variables, and because the purpose of this stage is just to obtain the matrix $A$, a very narrow range is allowed for decision variables in this first call, just $[0.97,1.03]$ around the initialization point.

2. A steady-state model simulation is performed, so that the independent terms $B$ are computed from the matrix $A$ and from a snapshot of decision variables and linear constraints.

3. For those constraints identified as linear, natural bounds are updated with independent terms $B$, so that the computed bounds can be applied by the SNOPT ${ }^{\circledR}$ routine: $I_{\text {lo/up }}^{\text {applied }}=I_{\text {lo/ up }}^{\text {natural }}-B$ 
4. Another SNOPT ${ }^{\circledR}$ function is called to perform the optimization. The full range for decision variables is allowed, because linear constraints fulfilment guarantees operation in the proper range without model convergence problems. Neither the optimization problem structure nor the matrix $A$ are computed again, but used from the first SNOPT ${ }^{\circledR}$ call.

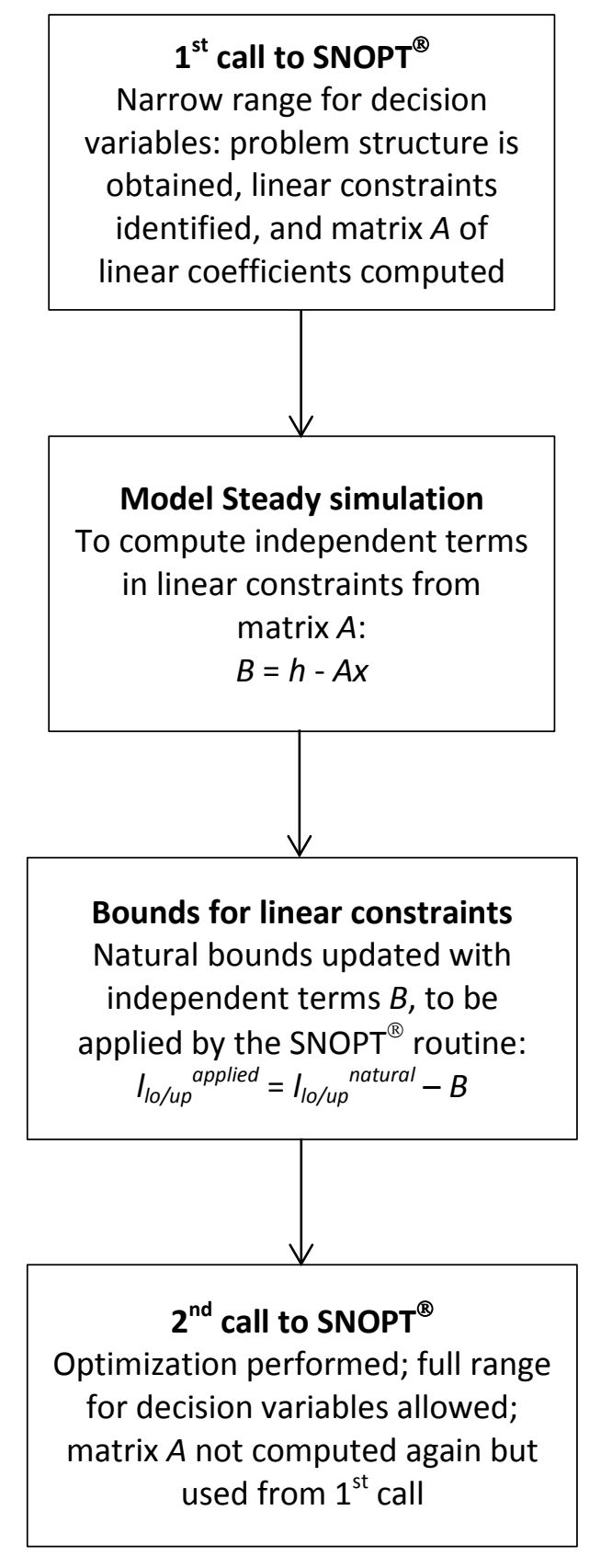

Figure 5.5. Scheme for SNOPT ${ }^{\circledR}$ two-stage call. 


\section{6- MODEL CALIBRATION AND VALIDATION}

\subsection{1- Model Calibration}

Previous to the automatic data reconciliation with optimization techniques for the global $\mathrm{H}_{2}$ network, a detailed manual calibration by simulation has been carried out for three different scenarios with the purpose of adjusting default values for model parameters, in particular solubility coefficients, as well as gaining insight regarding global sensitivities and trade-offs. The guidelines are stated below:

- A default value for the ratio $\Delta R_{\text {LIGH2 }}$ is specified according to Repsol ${ }^{\circledR}$ Technology Center (CTR) process knowledge, corroborating the information gathered from the design basis. This implies a default value for $\Delta R_{L I G}{ }^{s p}$;

- A value is specified for $\Delta R_{w L I G}$, according to average from historical laboratory analysis. The overall fitting presents low sensitivity to this parameter in medium-small consumer plants; moreover, it is poorly identifiable. Only for catalytic-reforming units and big consumer plants it has certain significance, due to the amount of light ends generated and the flow rate compensation dependence on molecular weight;

- Solubility coefficient $k_{\alpha H 2 L I G}^{S}$ has been tuned according to different criteria: i) value in accordance with hydrocarbon processed and pressure of operation in the separator; ii) reference for average $\mathrm{H}_{2}$ purity from historical laboratory analysis in the gas outlet from the corresponding separator, relative to both the current and average $\mathrm{H}_{2}$ purities in the HP separator;

Parameters $k_{\alpha H 2 L G G}^{S}$ and $k_{\text {wLIG }}^{S}$ have been individually tuned for each consumer plant, with the aim of fitting average laboratory compositions when operating at average $\mathrm{H}_{2}$ purity in the HP separator; when operating at higher/lower purity than the average in the HP separator, the target in the MP/LP separators was a higher/lower purity respectively; the same policy was observed to adjust the molecular weights. Not surprisingly, a certain correlation can be observed, and is expected because of the physical meaning of the coefficients, in the whole set of parameter values when compared according to the operating conditions in each separator regarding hydrocarbon processed and pressure. Temperature is not compared because it is usually the same for all separators, corresponding to the minimum achieved with air-cooled heat exchangers and depending mainly on ambient conditions.

Both $y^{\text {H2 }}$ and $w$ for a separator gas outlet are very influenced by the gas flow rate, therefore a poor fitting in these variables cannot be justified 
only because of the lack of model structural flexibility according to the specified ranges for parameters or a bad parameter tuning, but also because of flow measurement error and uncertainty in the compensation.

- A value is specified for $k_{\text {wLIG }}^{S}$ with the same criteria as for $k_{\text {aH2LIG. This }}^{S}$ parameter does not present a significant sensitivity, thus being less important;

- The remaining parameters $\left(\Delta R_{H 2}{ }^{s p}, k_{\text {gasHC }}^{S}\right)$ have been tuned without being previously bound, in order to achieve a good fitting for all available compensated measurement-model.

Ranges for model parameters and variables for the data reconciliation problem solved by optimization techniques were specified after this manual calibration had been performed, in consequence of a full understanding of the problem.

\subsection{2- Model Validation}

Validation for the global $\mathrm{H}_{2}$ network has been performed by automatic data reconciliation with optimization techniques. For the joint validation of the model and the data reconciliation, trends have been computed for periods of several days in different seasons; furthermore, also periods covering a change in the operating point have been chosen, to evaluate the results for two different scenarios as well as the transient state between them. Estimations for $\mathrm{H}_{2}$ consumption in reactors should also be evaluated according to process knowledge and expected trends as a function of the hydrocarbon processed, its sulphur content and the severity of the treatment.

Next, results for the validation stage will be presented. The off-line validation performed is accepted as correct according to the following facts:

- Even though the model suffers from overparameterization, consistent results in trends according to measured variables are obtained thanks to the allowed ranges in certain parameters. At the same time, model flexibility is enough to fit any set of real data, especially in the HP system which is the most determining and interesting to estimate.

- Lagrange multipliers for flow rate constraints in the reconciled solutions are usually only active for those linked to the HP system, not for the MP/LP ones. Therefore, the allowed ranges for parameters and variables do not force the solution to the extent that it is always guided in a certain direction depending on the MP/LP measurements, where more uncertainty exists due to: a small degree of redundancy regarding flow meters, no $\mathrm{H}_{2}$ purity analysers, together with more variability in the light ends molecular weight. Furthermore, similar results are obtained when reducing the weigh for all flow meters in the MP/LP systems one order or magnitude, maintaining equal those for the HP system. Also, similar results are obtained for broader parameter ranges. 
- Consistency checking in certain important relief-valves where no flow measurement is available has been performed according to the valve opening. In particular for the relief-valve in the $\mathrm{H} 4$ header sending excess under pressure control to the $\mathrm{H} 3$ header, which only opens occasionally in transient states due to changes of the $\mathrm{H} 4$ producer plant load. Although very broad limits have been fixed for the flow rate through the valve, the estimated value is coherent with the change in the raw measurements for the involved locally redundant flow rates along the transient state. When the valve is opened, one redundancy is lost, because the corresponding flow rate is not forced to zero; it is a good sign that the same consistent results are obtained before, during and after the transient state, irrespective of having one plus or one minus redundant measurement.

- Consistency checking for all important streams has been carried out according to the raw measurements trends, in particular for all make-up streams from headers to the consumer plants and the HP purge streams for both headers and consumer plants. It can be seen that errors are systematic, increasing or decreasing trends in transients are reproduced in the reconciled values, and similar results for the deviations in both sign and magnitude can be obtained in different periods of operation.

- When no redundancy neither local nor global exists for a certain flow meter, the model-compensated measurement fit should be perfect provided the model is flexible enough, and this is the solution found by the solver in such cases. However in the $\mathrm{H} 4$ header, being $\mathrm{H} 4$ the biggest producer plant, the total header imbalance when only considering the compensated measurements is lower than the sum of the absolute values of the residues model-compensated measurement for all the flow meters linked to the header; that is, the initial imbalance is distributed among the flow meters resulting in higher deviations model-reference than expected, thus a better solution with smaller errors could have been obtained for the isolated $\mathrm{H} 4$ header, but this is not the case in the global fitting according to all the network redundancies and constraints. The same occurs for the flow meters linked to the LPH. Provided the solvers performance is good, and experience verifies it, this fact can be seen as an additional support for the existence of systematic errors, taking into account that some of these flow meters have significant error contributions to the objective function to minimize.

In conclusion, it can be said that the model predictions are accurate enough and the target of the network state estimation by data reconciliation with the purpose of a subsequent optimization accomplished.

Finally, once coherent results have been assured in the previous steps and for the sake of completeness, a plant validation should be carried out in practical operation, in which certain make-up and HP purge flow rates were manipulated and changed, and effects over measured variables -in particular analysed HP purities-, compared among real process and model simulation. This is already planned for the following months. 


\section{Issues addressed}

Gas stream total molecular weight $w_{i}$ is very sensitive to light ends molecular weight $w^{L / G}$; specially for catalytic-reforming producer plants, where light ends generation is significant, outlet gas from the HP separator can show a high variability in $w_{i}$ when allowing broad ranges for $\Delta R_{w L I G}$ in the reactor, because both variables are closely related and nearly equal. Big step changes in consecutive periods from upper to lower bounds, when allowing a broad range, can be understood as a sign of poor parameter identifiability and sensitivity relative to other model parameters, see Fig. 5.6.

Assuming a Gaussian distribution for $\Delta R_{w L I G}$ in catalytic-reforming producer plants (e.g. $P 2$ ), then a proper range for the parameter would be [average \pm 2 . standard deviation] because $95 \%$ of the operating values will belong to it. However, this range has proved too much broad as can be seen in Fig. 5.6.

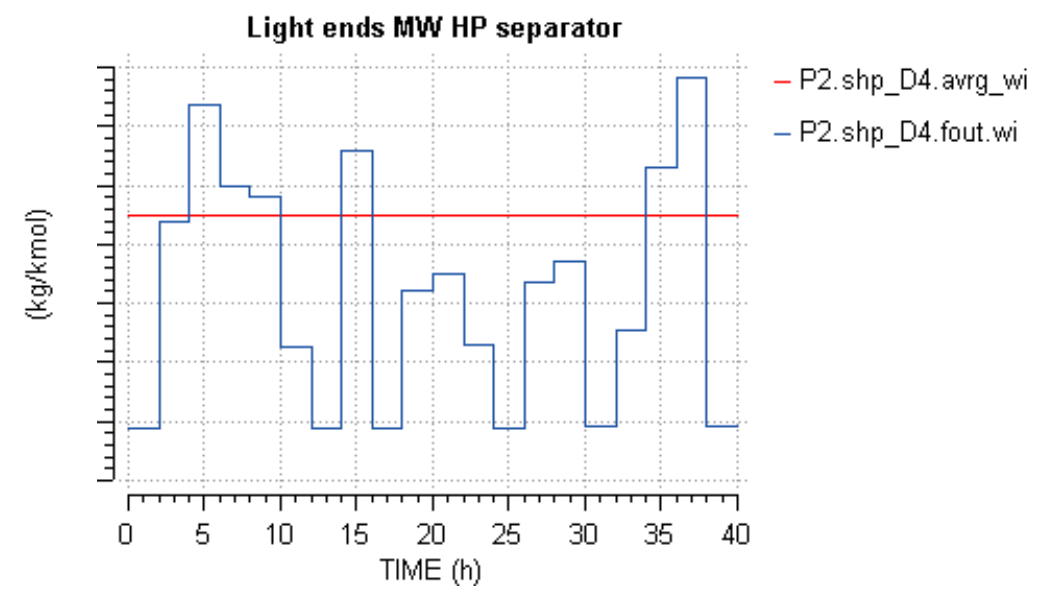

Figure 5.6. Molecular weight for light ends generated in catalytic-reforming producer plant.

This effect has been observed for important parameters, such as the molecular weight for light ends generated $\Delta R_{w L I G}$ for catalytic-reforming producer plants and big consumer plants, as well as the relative solubility coefficients $k_{\alpha H 2 L G}^{S}$ in the HP separators for all plants. Just by allowing a broad range, without changing the final configuration for the rest of the model and optimizer parameters, oscillations and inconsistent results are obtained which can also affect other variables.

The influence of a broad range for the relative solubility $k_{\alpha H 2 L G}^{S}$ over reconciled values for a certain consumer plant NC6 are shown in Fig. 5.7. This results in oscillations in the HP system $\mathrm{H}_{2}$ purity (analyser $A I_{-} 1 B$, whereas analyser $A I_{-} 1 A$ corresponds to total make-up), and as a consequence in the compensated recycle flow rate $\left(. d t F_{-} v c\right)$, reference for the reconciled model flow rate (.F), despite the smooth trend which can be observed for the raw measurement (.dtttmFop). Recycle flow rate is sometimes more prone to oscillations when no 
local redundancy is available, since it is an internal stream not influencing the inlet-outlet material balance of the HP system and the plant.

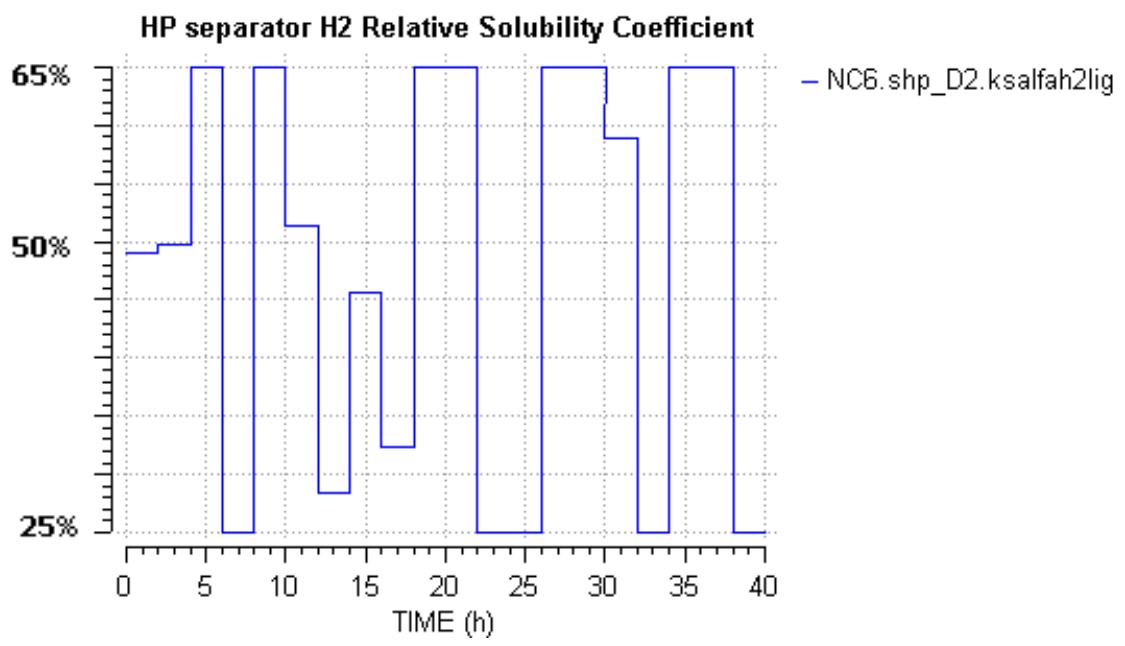

HP separator H2 purity Model-Experimental
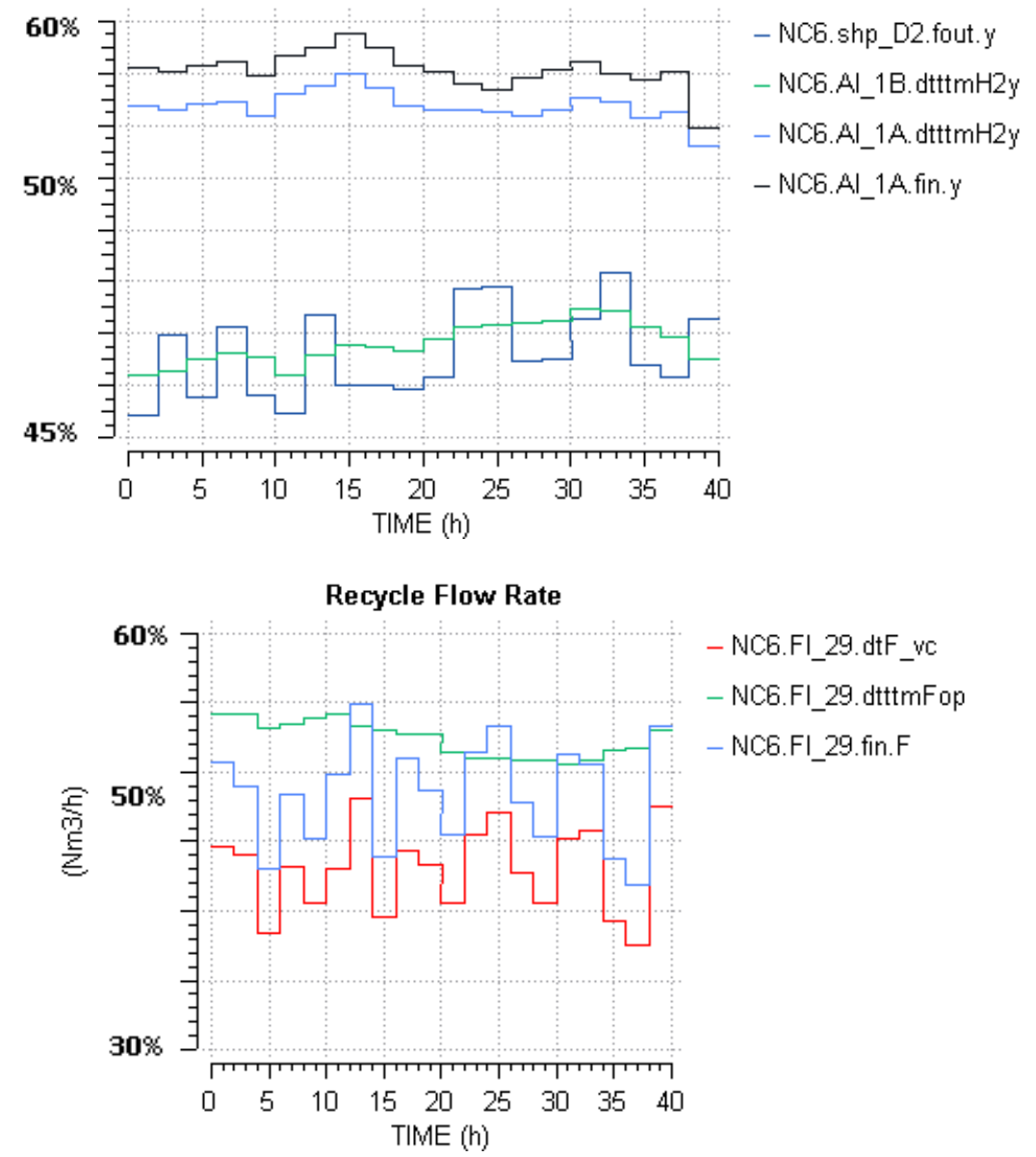

Figure 5.7. Effect of a broad range on the relative solubility coefficient $k_{\alpha H 2 L I G}^{S}$.

Available on-line measurements, available redundancies and meters uncertainty do not guarantee an accurate estimation of all model parameters, because the number of model degrees of freedom is higher than the number of on-line measurements, resulting in some 
unobservable parameters. Therefore, priority has been given to robustness and consistency in results not allowing too much flexibility to the optimization routine, at the expense of a reduced model flexibility to account for process variability in operating conditions.

Allowed range for flow rates, centred in the raw measurement according to factor $f_{i}^{\text {Uplo }}$, was also finely tuned in this stage. The resulting value was influenced by: trade-offs encountered in the fitting; imbalances in local redundant nodes; expected compensation factor $\beta_{i}^{\text {comp }}$ values; the pursued consistency and robustness.

\section{Analysis of results}

Data reconciliation provides consistent estimations of the plant variables; results for the most important variables are shown: total production in producer plants, make-up for the biggest consumer plants, interesting HP purges. Validation has been conducted by individual inspection of results for all measurements, as well as comparison with expected values according to operation and process knowledge. Consistency checking in trends has been carried out for: i) valve opening for certain important relief-valves without flow rate measurement; ii) raw measurement for important streams such as producer plants total production, make-up streams, HP purges and analysers. Next figures show flow rates and $\mathrm{H}_{2}$ purities for some of the most important streams, organized by plants and briefly commented before each subset.

All variables shown in Figures are scaled according to a certain percentage for the sake of confidentiality. Three flow rates are compared in each figure: the raw measurement $(d t F)$, the compensated raw measurement with pressure, temperature and molecular weight in operation, being the molecular weight a result of the model simulation $(F v c)$, and the model flow rate $(F m d l)$ solution of the reconciliation problem, whose reference for model fitting is the compensated raw measurement $(F v c)$. Regarding $\mathrm{H}_{2}$ purities, only for the HP system where they are measured, raw measurement $(d t y)$ is compared with the reconciled model purity $(y m d l)$.

Production of $\mathrm{H}_{2}$ in steam-reforming furnace producer plant $\mathrm{H} 4$ shows a good fitting according to the raw measurement trend. Absolute deviations are small in magnitude.

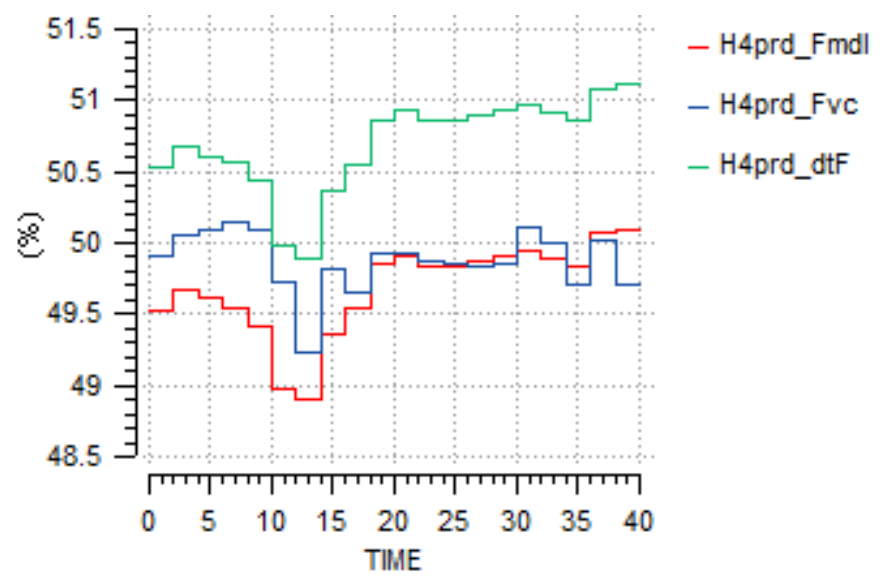

Figure 5.8. Production of $\mathrm{H}_{2}$ in steam-reforming furnace plant. 
Production of $\mathrm{H}_{2}$ in steam-reforming furnace producer plant $\mathrm{H} 3$ also shows a good fitting with respect to the raw measurement trend. Absolute deviations are small in magnitude. At the beginning of the trend, two points have higher deviations, implying a lack of robustness and consistency to a very small extent. Also at the beginning, the compensated raw measurement (Fvc) deviates from the raw measurement due to a change in pressure operating conditions, however reconciled model flow rate seems to follow the raw measurement.

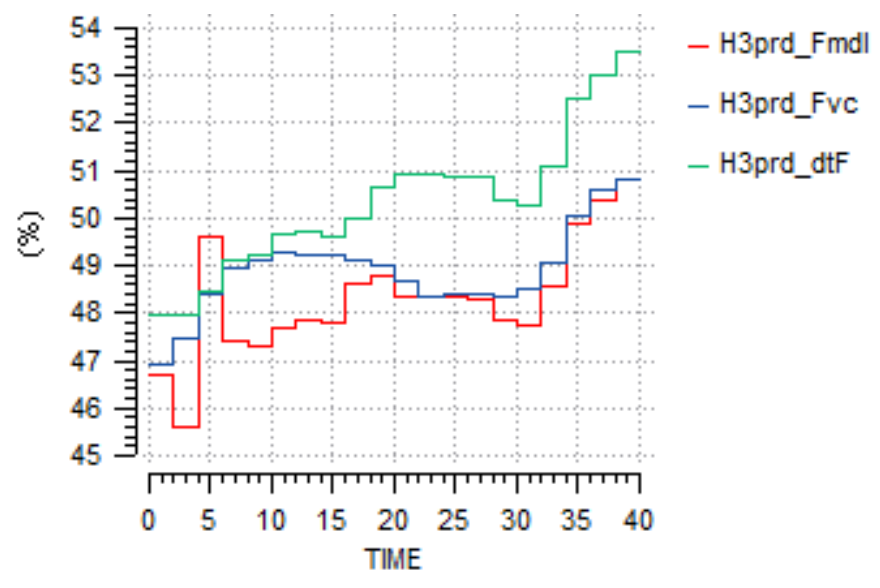

Figure 5.9. Production of $\mathrm{H}_{2}$ in steam-reforming furnace plant.

The fitting of the LPH purge to FG is good and consistent, despite a significant systematic error.

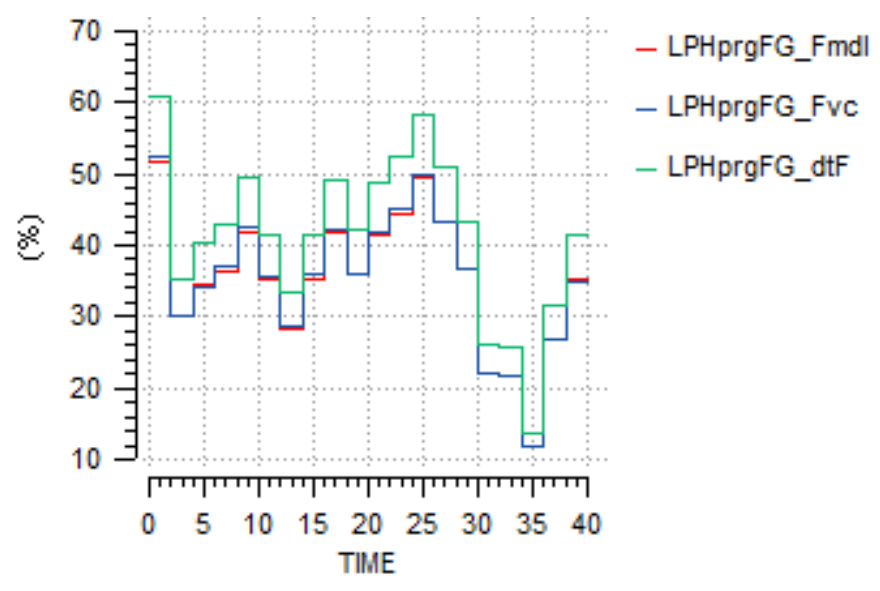

Figure 5.10. Purge to FG from the low-purity header LPH.

In the case of the relief of the $\mathrm{H} 4$ header to the $\mathrm{H} 3$ header, where a valve exists but no flow measurement, a good match can be seen between the valve opening along the transition and the estimated flow rate. Furthermore, although it cannot be seen in the Figure 5.11 because variables have been scaled, the absolute value in the estimated flow rate through the valve is equal to the difference in the gap between two local redundant raw measurements. It is also 
worthy to mention that when the valve is opened, one redundancy is lost in the global network, thus better reconciled results are expected when the valve is closed.
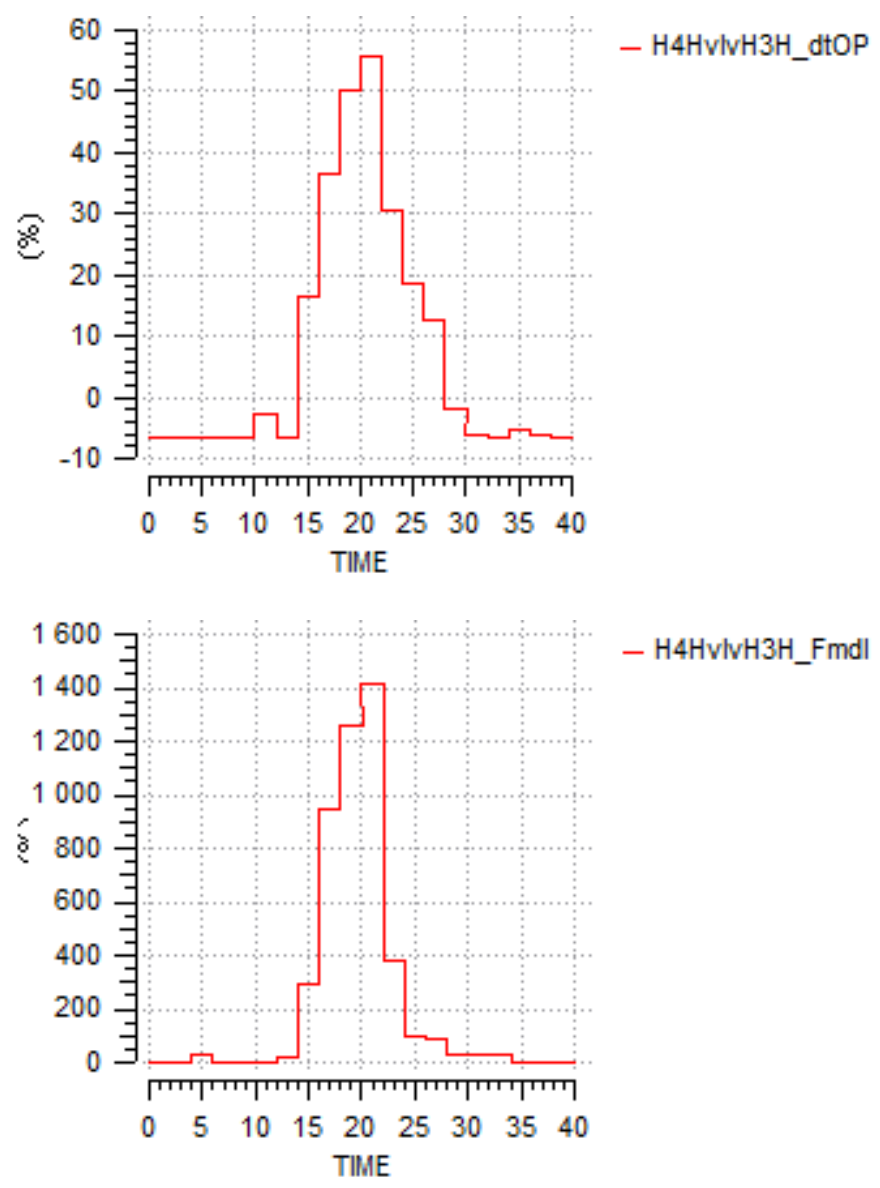

Figure 5.11. Valve opening and estimated flow rate for the relief from $\mathrm{H} 4$ header to $\mathrm{H} 3$ header.

G3 is one of the most important consumer plants. Consistent reconciled results can be observed for the $\mathrm{H} 4 \mathrm{H}$ make-up (H4Hmkp), the H3H make-up (H3Hmkp), the LPH make-up (LPHmkp), the inlet to membranes (Zin), the permeate from membranes (Zprmt), the HP purge from membranes (Zprg), the HP $\mathrm{H}_{2}$ purity $(H P y)$, the MP purge (MPprg), and the LP purge (LPprg). 

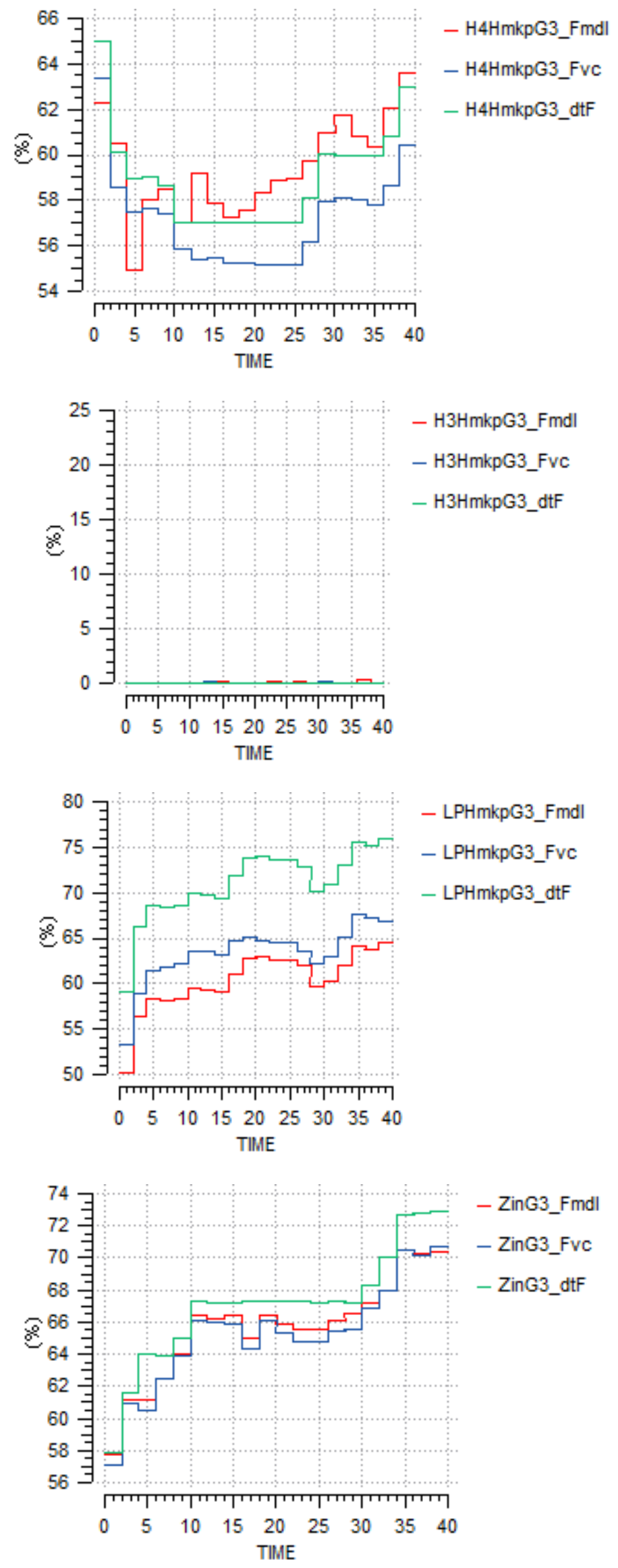

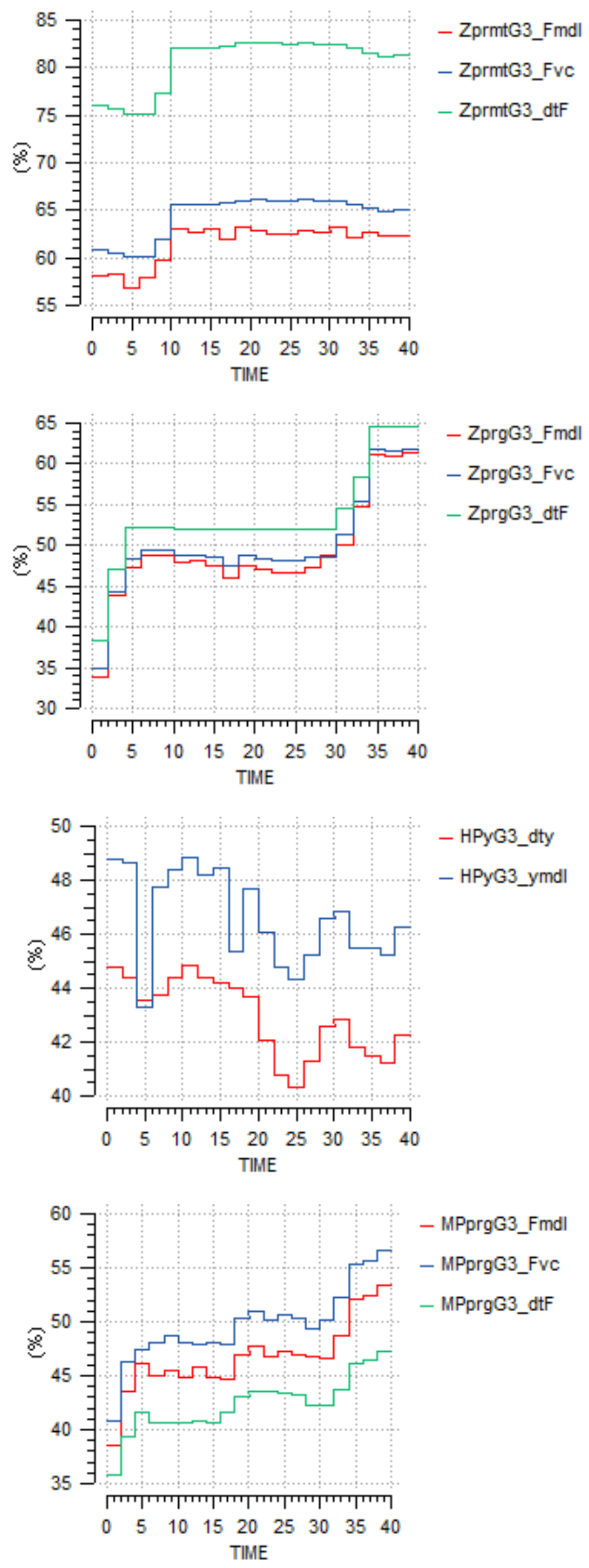


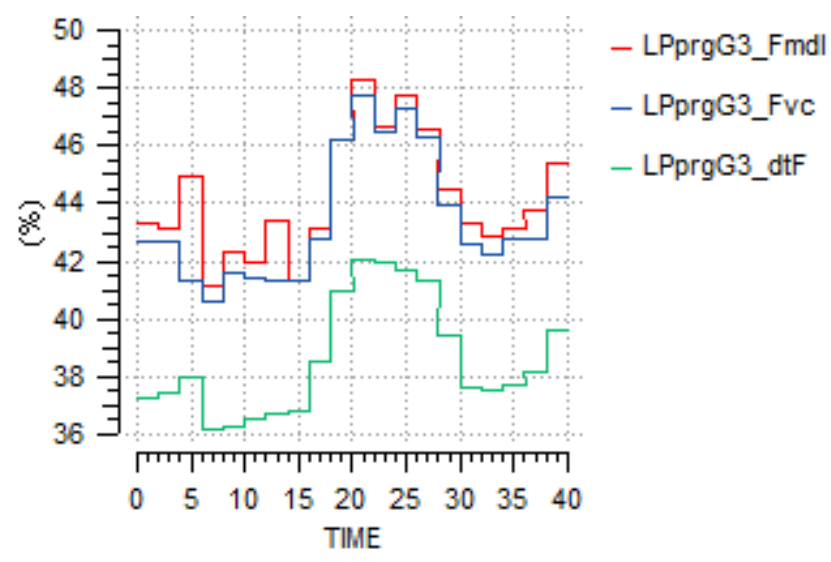

Figure 5.12. Reconciled solutions vs. measurements for consumer plant G3.

G4 is one of the most important consumer plants. Consistent reconciled results can be observed for the $\mathrm{H} 4 \mathrm{H}$ make-up (H4Hmkp), the H3H make-up ( $\mathrm{H} 3 \mathrm{Hm} m \mathrm{kp}$ ) which is zero, the LPH make-up (LPHmkp), the HP purge is zero and has not been represented, the total reactor gas inlet $\mathrm{H}_{2}$ purity $(R T y)$, the HP $\mathrm{H}_{2}$ purity $(H P y)$, the MP purge (MPprg), and the LP purge (LPprg).

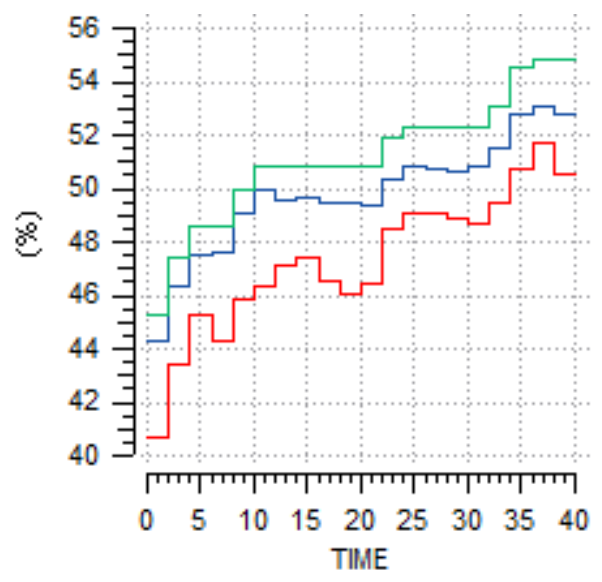

- H4HmkpG4_Fmdl

- H4HmkpG4_Fvc

$-\mathrm{H} 4 \mathrm{HmkpG} 4$ dtF

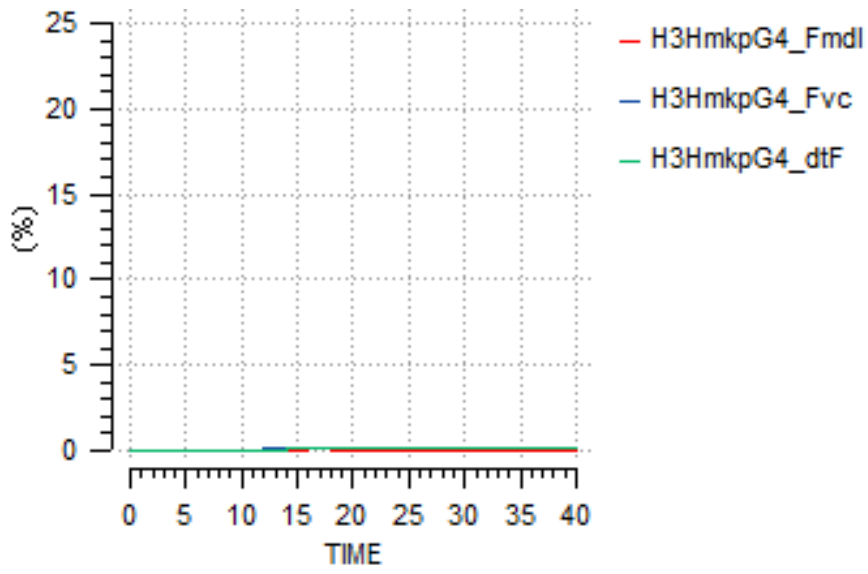



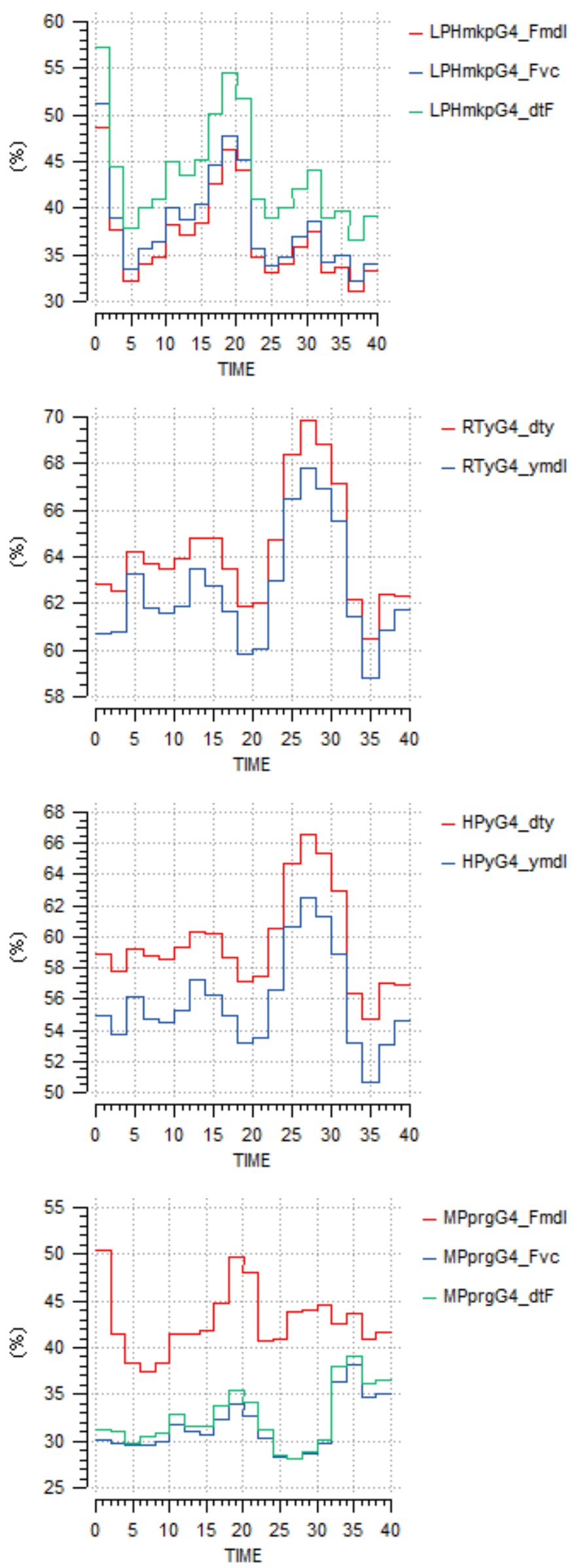


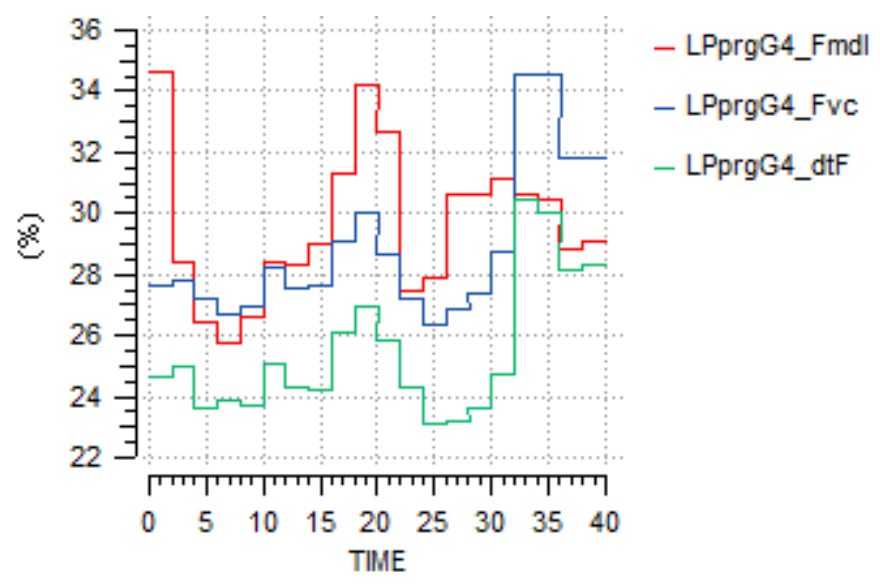

Figure 5.13. Reconciled solutions vs. measurements for consumer plant G4.

HD3 is one of the most important consumer plants. Consistent reconciled results can be observed for the $\mathrm{H} 4 \mathrm{H}$ make-up (H4Hmkp), the H3H make-up (H3Hmkp), the LPH make-up (LPHmkp), the HP purge (HPprg), the total reactor gas inlet $\mathrm{H}_{2}$ purity $(R T y)$, the $\mathrm{HP}_{2}$ purity $(H P y)$, the MP purge (MPprg), and the LP purge (LPprg).
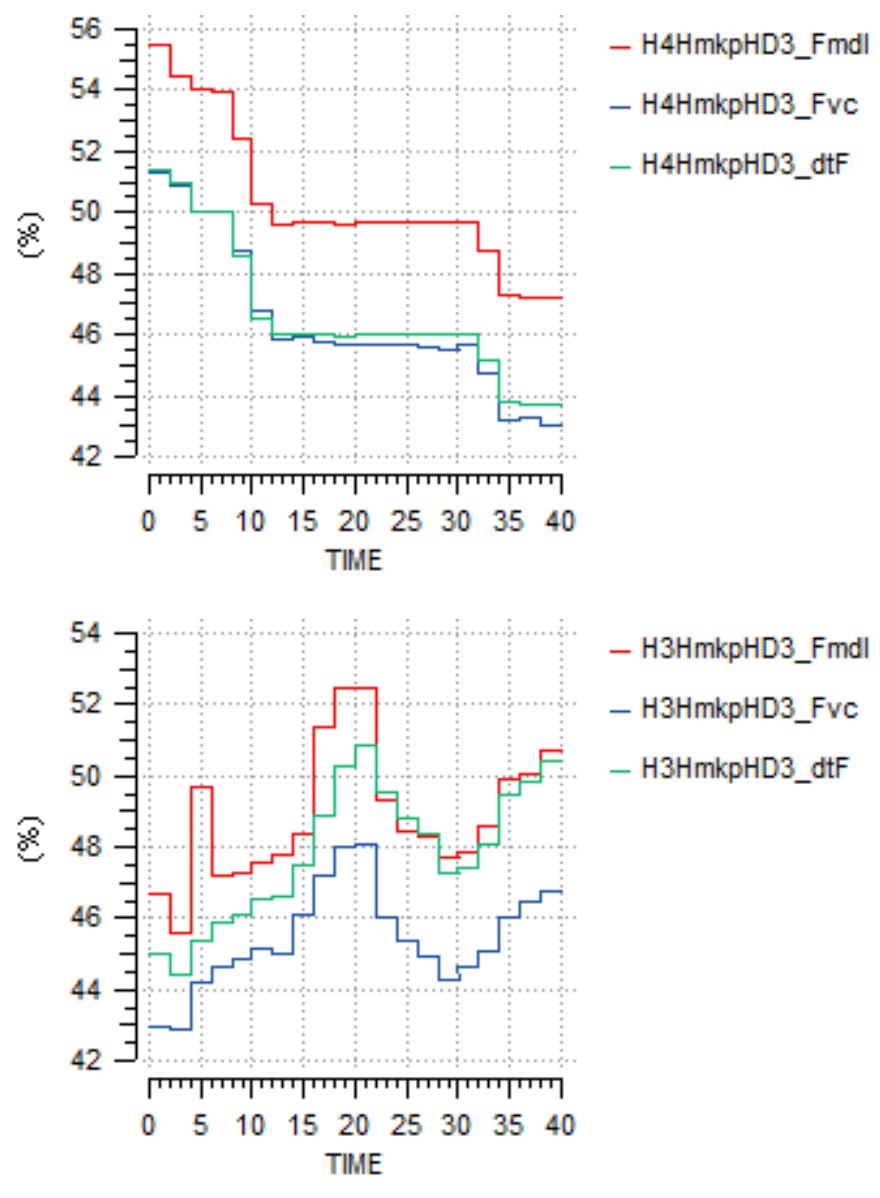

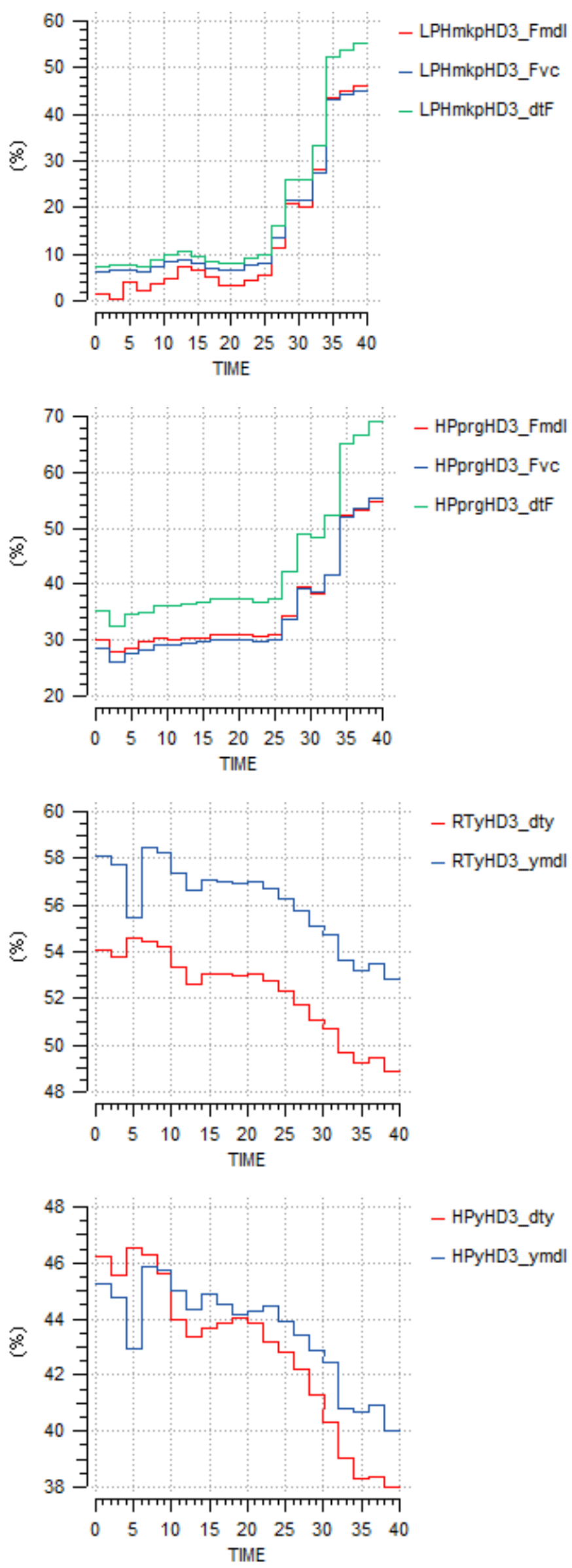

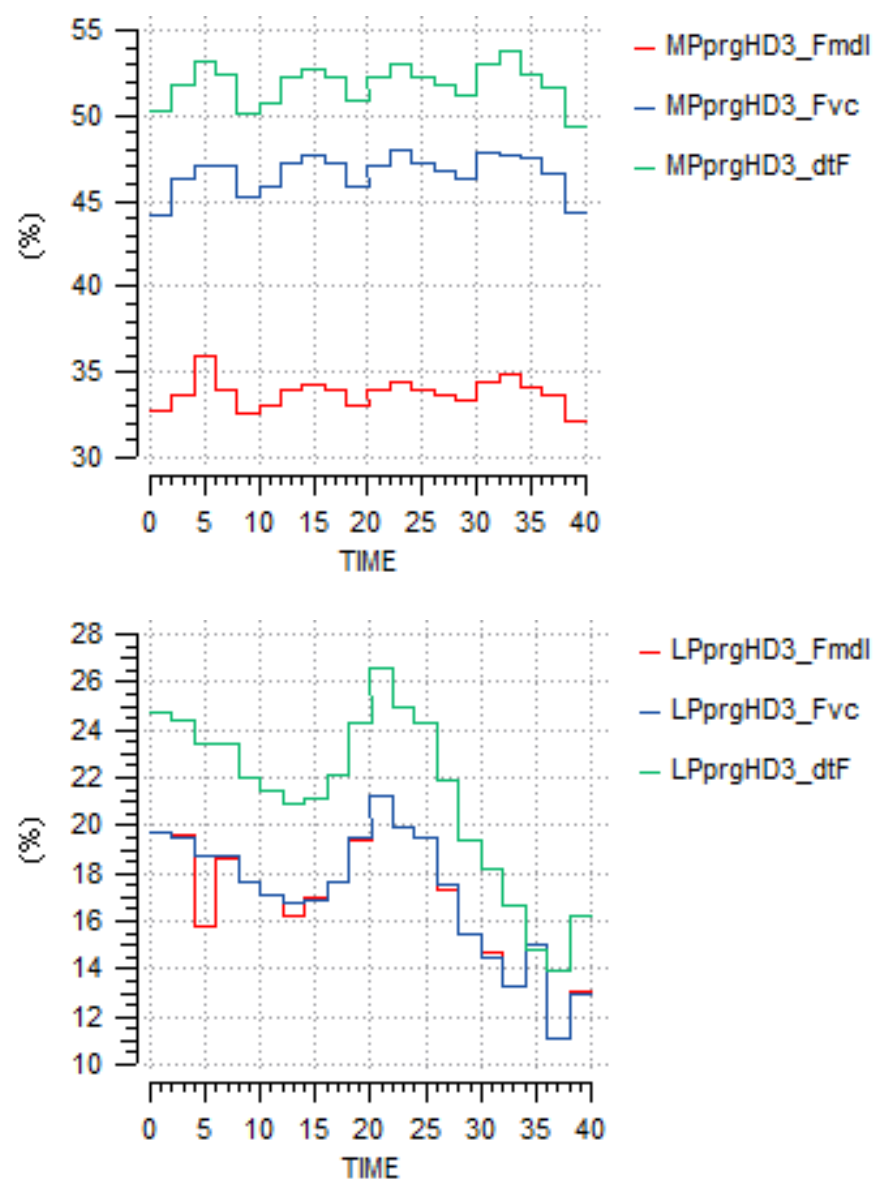

Figure 5.14. Reconciled solutions vs. measurements for consumer plant HD3.

$\mathrm{G} 1$ is also one of the most important consumer plants. Consistent reconciled results can be observed for the $\mathrm{H} 4 \mathrm{H}$ make-up (H4Hmkp) which is null, the LPH make-up (LPHmkp), the HP purge (HPprg), the HP $\mathrm{H}_{2}$ purity (HPy), the MP purge (MPprg), and the LP purge (LPprg). In this case, the LPH make-up (LPHmkp) presents certain oscillations in the compensated flow measurement $(F v c)$ due to the model estimated molecular weight, since no on-line analyser is available in the upstream plant where the low-purity $\mathrm{H}_{2}$ is generated, together with the fact that the allowed range for certain solubility and reactor model parameters is not as narrow as to avoid this small inconsistency. However, a systematic error can be observed, and this particular trend is not detrimental to other flow measurements fit. 

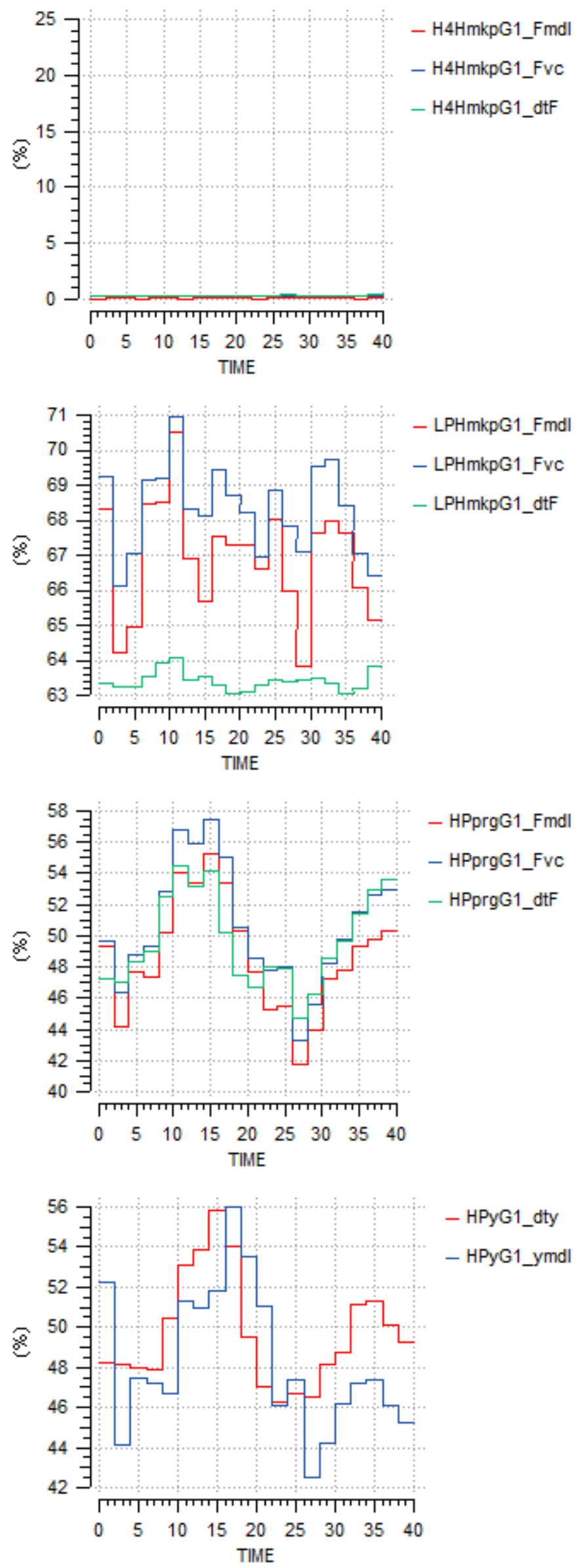

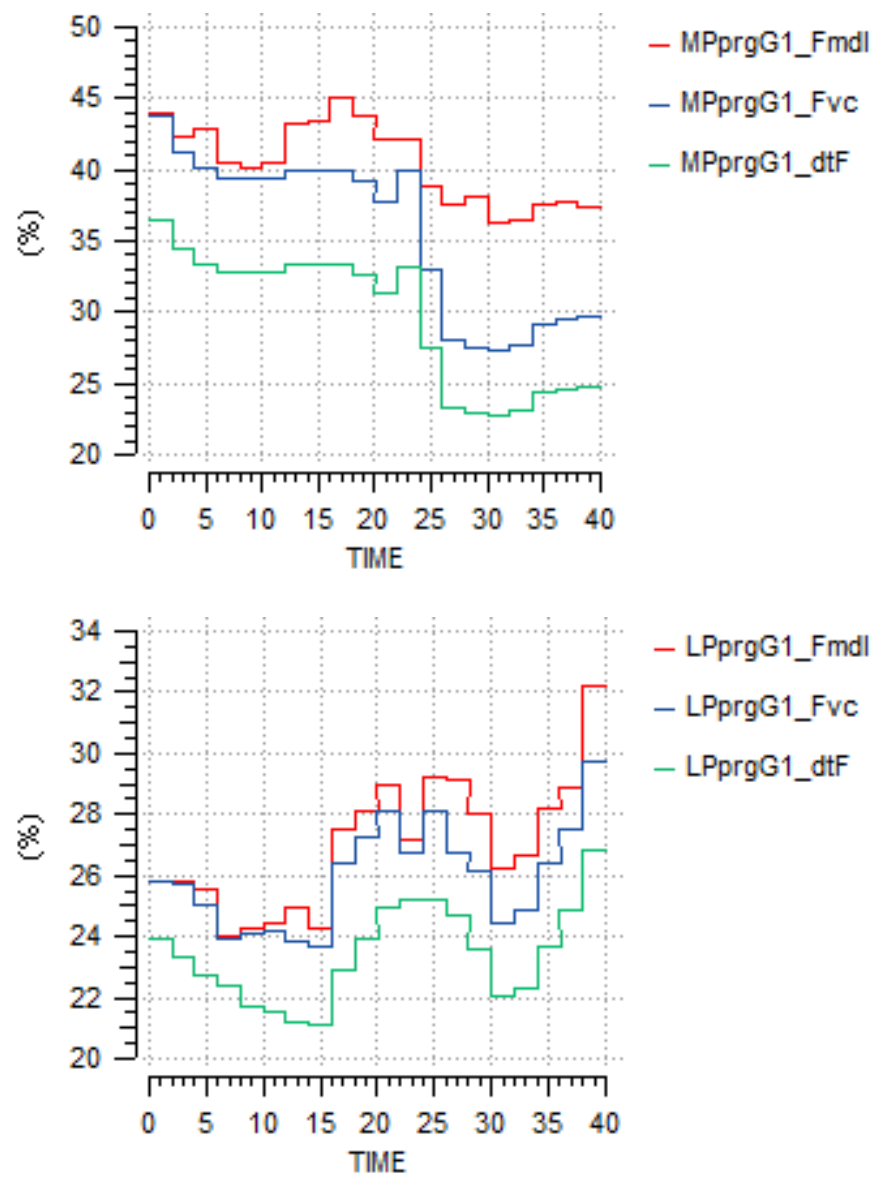

Figure 5.15. Reconciled solutions vs. measurements for consumer plant G1.

Finally, table 5.6 contains the total number of analysers and flowmeters available, as well as information about how many analysers are not working, how many flowmeters have been disregarded due to unacceptable gross errors, and the number of flowmeters with a compensation factor $\beta_{i}^{\text {comp }}$ not close to 1 .

\begin{tabular}{lc}
\hline On-line Measurements & No meters \\
\hline Total Analysers (AI) & 20 \\
Total Flowmeters (FI) & 176 \\
Disregarded [Al: off / Fl: gross error] & $6 / 16$ \\
Flowmeter $\beta_{i}^{\text {comp }} \notin(0.80,1.20)$ & 21 \\
Flowmeter $\beta_{i}^{\text {comp }} \notin(0.85,1.15)$ & 39 \\
\hline
\end{tabular}

Table 5.6. Total on-line flowmeter and analyser measurements. 
Distribution of $\mathrm{Fl}$ meters within different ranges is obtained, for the residues compensated measurement-reconciled value relative to: i) span; ii) standard deviation; iii) raw measurement. Also an average value for the relative residue is obtained in the three cases. Flowmeters with gross errors, which are not considered in the data reconciliation, are not included in the statistical analysis either; neither flowmeters whose raw measurement is under a certain threshold, $0.01 \cdot \mathrm{span}+35\left(\mathrm{Nm}^{3} / \mathrm{h}\right)$, in order to avoid misleading contributions. A typical distribution of flowmeters regarding the relative error w.r.t. span is shown in Table 5.7, and also average values for the three relative residues; similar results are obtained for the other different scenarios.

$$
\begin{aligned}
& R_{\text {span }}^{\text {average }}=\sum_{i=1}^{N} \frac{a b s\left(F_{i}^{\text {measured }} \cdot \beta_{i}^{\text {comp }}-F_{i}^{\text {model }}\right)}{\operatorname{span}_{i}} \\
& R_{\text {stdv }}^{\text {average }}=\sum_{i=1}^{N} \frac{a b s\left(F_{i}^{\text {measured }} \cdot \beta_{i}^{\text {comp }}-F_{i}^{\text {model }}\right)}{\sigma_{i}} \\
& R_{\text {meas }}^{\text {average }}=\sum_{i=1}^{N} \frac{a b s\left(F_{i}^{\text {measured }} \cdot \beta_{i}^{\text {comp }}-F_{i}^{\text {model }}\right)}{F_{i}^{\text {measured }}}
\end{aligned}
$$

\begin{tabular}{lcl}
\hline & Average / & Units \\
Residue & № Fl in range & \\
& \\
\hline
\end{tabular}

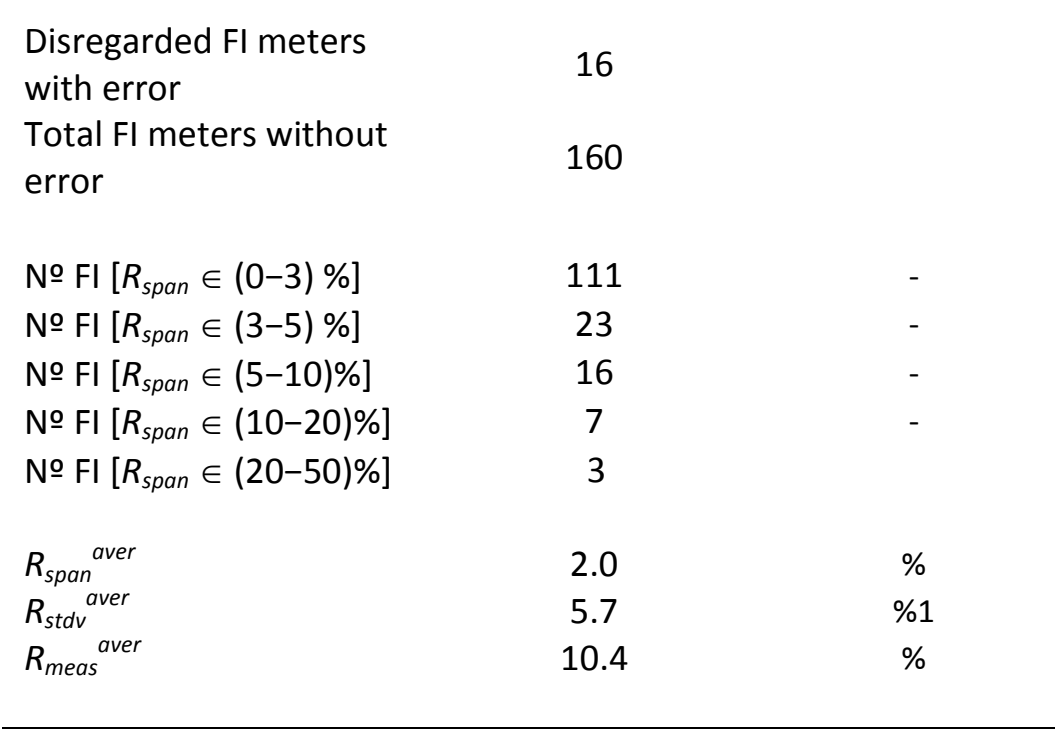

Table 5.7. Relative residues compensated measurement-reconciled value.

To conclude the analysis of results, some remarks are pointed out regarding the particular $\mathrm{H}_{2}$ network problem solved, although most probably can be generalised to similar topologies: 
- Main interactions at the network scope take place in the LPH, where most consumer plants pour their $\mathrm{H}_{2}$ in excess and which serves as make-up for the three most important consumer plants, thus being further reused. Besides, main uncertainties in composition correspond to the LPH, because of: i) lack of on-line analysers; ii) different composition for the different stretches according to inlet and outlet flow rates. In headers linked to producer plants, one redundant flow measurement is available and composition is accurately measured without uncertainty.

In the achieved optima, usually all the significant non-zero Lagrange multipliers correspond either to analysers or to flowmeters related with the LPH or make-up and purge streams in the HP system. Therefore, in spite of the allowed ranges for certain parameters, model structure is flexible enough to fit MP/LP purges, which do not determine strongly the solution reached, whereas trade-offs arise in the LPH and HP system where both redundancy and also less uncertainty regarding composition in comparison with the MP/LP systems exist, assisting in the fitting.

- The analysis has also focus on the allowed tolerance for analyser measurement errors. In general, analyser tolerances are very determining for the solution achieved regarding flow rates, due to the high sensitivity of $\mathrm{H}_{2}$ purity to flow rates as aforementioned. Accurate on-line measurements for $\mathrm{H}_{2}$ purity in the HP system $\mathrm{Y}_{\mathrm{HP}}{ }^{\mathrm{H} 2}$ are important, especially for big consumer and producer plants. Mainly two types of on-line analysers are available, those based on gas chromatography where a sampling is needed and the measurement has a delay of several minutes, and those based on density measurement, where the meter is installed on the pipe.

Furthermore, from the global network fitting viewpoint, the study has shown that an accurate $y_{H P}{ }^{H 2}$ measurement in the catalytic-reforming producer plants is very relevant for a good fitting, since it is one of the most important sources of light ends and moreover, spread through the whole network. In this regard, an estimation of $y_{H P}{ }^{H 2}$ for the catalyticreforming units based on a density meter has proved not appropriate, with frequent errors up to $10-20 \%$. Density is equivalent to gas molecular weight $w$ with the ideal gas model and known conditions of pressure and temperature, therefore the estimation of $y_{H P}{ }^{H 2}$ with the density meter is based on the relation between $y^{H 2}$ and the total gas molecular weight $w$ assuming a constant average $w^{L / G}$, deriving errors in the measurement of $y_{H P}^{H 2}$ from the uncertainty in the $w^{L I G}$ generated, together with its relatively broad range of variability. 


\section{7- CONCLUSIONS}

The well-known technique of process state estimation by means of the minimization of an objective function composed of model-measurement deviations, WLS weighted least squares, benefits from dealing with all the available on-line information. The problem addressed is challenging due to uncertainty: error prone flowmeters, lack of on-line measurements for $\mathrm{H}_{2}$ purity and gas composition, mixtures complexity with strong influence over flow measurements compensation, and significant process variability. A rational approach has been applied to the $\mathrm{H}_{2}$ network according to parameter sensitivities and problems encountered. Conclusions are summarised next:

- The underlying model based on conservation laws has enabled to detect and disregard instruments affected by gross errors in an off-line analysis by simple inspection, because gross errors are persistent in time, and constant in sign and approximately magnitude (systematic).

- A rational methodology has been followed for model calibration and validation. Firstly, calibration was performed for isolated plants to a certain extent, using as reference available historical laboratory data and design data. Secondly, manual adjustment in simulation enabled to apprehend the global problem; important sensitivities and trade-offs were studied, enabling to focus the attention on those model and configuration parameters more relevant. Finally, the model and optimizer parameters and ranges were finely tuned for a global fitting of the network. Validation was accomplished by comparison with raw measurement trends for all the most important variables along different periods, with satisfactory results.

- Model parameters and bounds have been tuned to achieve good performance, guaranteeing consistency and robustness of results at the expense of a more reduced flexibility than expected according to process variability in certain model parameters (mainly the solubility parameters), although not compromising the accuracy of the results. Further improvements will be difficult to accomplish due to: i) inescapable local optima; ii) a certain degree of overparameterization and poor identifiability; iii) uncertainty in on-line measurements.

Although unbiased estimates cannot be guaranteed with the WLS technique in the presence of non-random errors, potential drift and bias errors in flowmeters $\mathrm{FI}$ and analysers Al do not affect the reconciled results badly; consistency according to expected trends in important variables is achieved provided allowed ranges are finely tuned, and an improved estimation is obtained by taken advantage of all redundancies. Narrow ranges for certain $\mathrm{Fl}$ and $\mathrm{Al}$ have proved determining, again due to multiple local optima and interactions. Different treatments for different variables according to their type (Al/FI) and sensitivity have been applied; accepted tolerance in measurements has proved important in the data reconciliation step, in particular allowed range for Als measurement 
error, because due to their high sensitivity this tolerance determines to a great extent the achieved fit in the whole set of Fls. The difficulty to balance heterogeneous addends in the objective function, those corresponding to analysers and flowmeters, has been tackled by using purely non-linear constraints.

- A dynamic data reconciliation approach would not be recommended to step further. Dynamic reconciliation is an alternative in order to refine results, taking into account accumulation effects due to recycle streams and transport delays. Averages values for a period of several minutes should be selected for data reconciliation instead of the current two-hour averages. Nevertheless, current robustness and consistency in results will probably not make up for the effort to develop a dynamic model and dynamic optimization, together with the increased execution time. In fact, trends in raw measurements can be reproduced with the available steady-state model, and coherent results for different periods of operation result in sufficient reliability with the current approach. 



\section{CHAPTER 6}

\section{HydRogen Network Optimal Redistribution}

\section{1- INTRODUCTION}

Resource saving is a major concern for process plants in general, and in particular for oil refineries due to the low profit margins and large amounts of raw materials and energy involved. Efficiency in the use of hydrogen as a raw material in the oil refinery of Petronor ${ }^{\circledR}$ (Muskiz, Vizcaya), belonging to Repsol ${ }^{\circledR}$ group, is considered.

An efficient use of $\mathrm{H}_{2}$ in the daily operation is desired, because $\mathrm{H}_{2}$ has gained significant importance in the refinery global economic balance due to several reasons:

- In recent years heavier crude oils are being processed, therefore with a higher content of sulphur and nitrogen compounds leading to higher $\mathrm{H}_{2}$ consumption;

- Environmental regulations are progressively more strict, experiencing $\mathrm{H}_{2}$ requirements a steady increase;

- Not only $\mathrm{H}_{2}$ production cost is high (very endothermic reaction at high temperature in the steam-reforming furnaces from natural gas), but also the economic penalty is even higher in scenarios where $\mathrm{H}_{2}$ production capacity is bottleneck for oil processing capacity;

- Decisions regarding $\mathrm{H}_{2}$ management are complex as many plants and operating constraints are involved in the network operation with a high degree of interrelation, not only from an optimality viewpoint but also from a practical viewpoint because several operators at different control rooms are typically in charge; thus a quantitative criterion for decision support can prove very valuable;

- Feedstock usually changes every two-three days, and also certain product specifications can vary according to the global management of the refinery. As a consequence, scenarios regarding $\mathrm{H}_{2}$ consumption in individual consumer plants can experience frequent significant changes, making a tool intended for real-time decision-making purposes interesting; 
The approach to deal with the $\mathrm{H}_{2}$ network optimal management is driven by an operational framework where $\mathrm{H}_{2}$ production must always exceed $\mathrm{H}_{2}$ consumption, because $\mathrm{H}_{2}$ deficit is extremely damaging for catalysts being catalysts very expensive. As $\mathrm{H}_{2}$ accumulation in a buffer vessel is not possible, besides the slow dynamic for $\mathrm{H}_{2}$ production in the furnaces of the intended steam-reforming plants, the target of $\mathrm{H}_{2}$ production minimization has to be achieved by means of:

- a good dynamic fit of $\mathrm{H}_{2}$ production to consumption, in order to minimize excess sent to the Fuel Gas network under pressure control in the headers. This is a controllability problem, addressed with a MPC designed and commissioned by the Petronor ${ }^{\circledR}$ Advanced Control Department, in cooperation with the Production Department. The purpose is to control certain determining specifications regarding the $\mathrm{H}_{2}$ network, therefore avoiding/reducing the gaps providing for margin in case of disturbances; the MPC accounts for the most significant interrelations in the multivariable control of the LPH header, where excess from consumers is poured and which serves as make-up for certain consumer plants.

The reduction of the minimum margin allowed for in the purge from the LPH to FG, which is the main contribution to inefficiency in the network $\mathrm{H}_{2}$ management, is beyond the scope of this work and cannot be dealt with a RTO approach.

- a better $\mathrm{H}_{2}$ redistribution from $\mathrm{H}_{2}$ producer to $\mathrm{H}_{2}$ consumer plants. This problem is of a different nature, acting on a slower time-scale in an upper layer in the hierarchy than the aforementioned advanced regulatory control (MPC), and is addressed with an RTO approach. The decisions to be taken in the $\mathrm{H}_{2}$ network optimal management are:

- which plants must produce $\mathrm{H}_{2}$ and their production rates;

- which combination of make-up flows from each header must provide $\mathrm{H}_{2}$ to each consumer plant;

- which must be the operating point for membrane units, and in general for high-pressure HP purges to FG in certain consumer plants.

This is the problem tackled in this Chapter, based on the proposed model and the actual network state obtained by solving the data reconciliation problem.

The model and the optimization capabilities have been further exploited in two applications, briefly explained at the end of the Chapter. The first one is a study made in collaboration with the Repsol ${ }^{\circledR}$ Technology Center -CTR- in Móstoles (Madrid), with the aim of assessing different changes in the topological design of the Petronor ${ }^{\circledR}$ refinery $\mathrm{H}_{2}$ network, steered towards $\mathrm{H}_{2}$ production minimization and cost reduction. The second one is a What-If simulation used to experiment the effects over $\mathrm{H}_{2}$ efficiency of different operational strategies; complemented with a set of defined resource efficiency indicators (REIs) intended for on-line decision support regarding the $\mathrm{H}_{2}$ network management, it can be a useful tool. 


\section{2- APPROACH AND STATE OF THE ART}

RTO refers to a technique based on mathematical optimization for decision making purposes regarding problems in the time-scale of hours. In the well-known general hierarchy for control and decision making in a plant, organized according to a progressive refinement of the timescale and also acting at different scopes ranging from plantwide optimization at the top to single PID loops at the bottom passing through units in the middle regarding the advanced control applications, the RTO belongs to a cascade of interconnected problems: Planning (weeks) - Scheduling (days) - RTO (hours) - MPC (minutes) - Distributed Regulatory Control System (seconds).

For an overview and assessment of current practice in RTO, Darby and Nicholson et al. (2011) can be seen. A good description of each layer is provided there, and repeated here. Planning is concerned with "what and how" based on economics and forecasts, and answers such questions as what feedstocks to purchase, which products to make, and how much of each product to make. In most all refineries and larger chemical plants, a LP or successive SLP is used for planning and is based on an overall plant profit objective function. Scheduling is concerned with "when". Scheduling addresses the timing of actions and events necessary to execute the chosen plan, with the key consideration being feasibility. Scheduling deals with such issues as the timing of the deliveries of feeds, product liftings and operating mode changes, and avoiding storage problems, overflow or shortage. A range of tools are used across the industry for scheduling, from spreadsheets to tools involving simulation models, rules, and optimization. The RTO layer and the levels below it execute in real-time, although at different intervals, and there is automatic, continual feedback from the process. At the planning and scheduling levels, feedback and model updating is not automatic and is performed intermittently. RTO handles the implementation of business decisions in real time based on a calibrated non- linear steady-state or dynamic model, with model detail that the planning model does not have. RTO is implemented where economically justified and is typically formulated based on a profit function of the plant. The MPC(s) deals with the dynamic control of the plant and provides some amount of optimization capability. Optimal operation typically resides at the intersection of multiple constraints. As a result, MPC has often proved necessary to move the plant to, and maintain operation at, the steady-state optimum between successive executions of the RTO. RTO values are directly passed or translated to values or parameters of the MPC.

The practice of implementing real-time optimization (RTO) using a rigorous steady-state model, in conjunction with model predictive control (MPC), dates back to the late 1980s. Since then, numerous projects have been implemented in refinery and chemical plants, and RTO has received significant attention in the industrial and academic literature. Potential opportunities for successful applications are based on a combination of different factors, such as robust and efficient computational tools, user-friendly and reliable software, skilled users, and organizational structures promoting the collaborative efforts (Darby, Nicholson et al., 2011). Examples of successful implementation of model-based optimization from the petro and chemical industry can be seen in the literature. 
Bussani, Chiari, Grottoli, Pierucci et al. (1995) report the use of a detailed model for optimization of a hydrogen plant. Mercangöz and Doyle (2008) apply RTO to the pulp mill benchmark problem. ljaz, Ati and Mahalec (2013) work in heat exchanger network simulation, data reconciliation and optimization. Mendoza et al. (2013) apply the RTO to an industrialscale vapor recompression distillation process, with model validation and analysis. Serralunga, Mussati and Aguirre (2013) present a model adaptation for RTO in energy systems. Sildir et al. (2013) present a plant-wide hierarchical optimization and control of an industrial hydrocracking process. Faber, Li, Li and Wozny (2006) work in data reconciliation for RTO of an industrial coke-oven-gas purification process. Mandur and Budman (2015) present simultaneous model identification and optimization in presence of model-plant mismatch. Xenos, Thornhill et al. (2015) present an industrial case study of RTO for optimization of a network of compressors in parallel in chemical plants.

Serralunga, Aguirre, Mussati (2014) include disjunctions in RTO. Traditionally, RTO has used nonlinear continuous formulations to model the process. Mixed-integer formulations have not been used in RTO, because of the need of a fast, and because many discrete decisions, such as startups or shutdowns, are taken with less frequency in a scheduling layer. Their work proposes the use of disjunctions in RTO models, listing a series of examples of discrete decisions, different to startups or shutdowns, that can be addressed by RTO. Two model adaptation approaches, the two-step approach and the modifier adaptation strategy, are revised and modified to make them suitable for RTO with discrete decisions. Some common techniques used in RTO, such as filtering the optimal inputs, are also analyzed and adapted for a formulation with disjunctions.

Jun, Zhi-zhong and Run-da (2015) work on RTO based on SCFO for gold cyanidation leaching process. To solve inevitable plant-model mismatch and process disturbance in practice, an RTO strategy based on SCFO (Sufficient Conditions for Feasibility and Optimality) is proposed, where the SCFO are used to enforce the desirable properties (both feasibility and optimality) of the solutions resulted from the traditional RTO methods. The simulation results show that compared with the traditional RTO methods, there is a significant improvement of production cost by enforcing the SCFO under model-parameter uncertainty, model-structure uncertainty and unknown process disturbance.

The RTO approach has been selected due to the complex and multivariable nature of all the interactions among plants at the network scope.

An alternative approach to RTO like model-based predictive control MPC presents clear advantages for the on-line optimal operation (Skogestad et al., 2014); the MPC technique just needs robust relations between variables and has good performance in spite of measurement drift errors, thus being easier to implement and maintain. However it can fail to provide a complete handling of all decision variables, non-linear constraints and non-linear trade-offs. The model-based predictive control MPC technique is one of the most successful approaches for multivariable optimization and constraint management in industrial practice, which implements in closed-loop the first step of the optimal solution trajectory for the minimization of a certain objective function, subject to physical constraints, and using a dynamic process 
model. Usually a linear model is employed, although a non-linear one is also possible in nonlinear MPC. The main drawback in this case for the practical implementation of a linear MPC, the widespread commercially available DMC, is the strongly non-linear and multivariable nature of the problem, with significant interactions regarding the LPH enabling to take advantage of $\mathrm{H}_{2}$ excess. For example, when the HP purge flow rate for a consumer plant is increased, $\mathrm{H}_{2}$ purity in the HP system increases as a consequence of a higher removal of lightends in the purge stream than inlet of light-ends in the increased (according to pressure control=material balance) make-up stream; however, when it is the LPH make-up which is under pressure control, and if the LPH purity changes from medium values to lower values (below the HP system purity), then it occurs that an increase in the HP purge flow rate results in a decrease in the HP system purity. This situation does not occur with steam-reforming furnace producer plants, because usually their purity is much higher than the HP system one; moreover, these producer plants are more isolated and the dependence with the global network takes place typically only in the same one direction. However, strong multivariable interactions occur within the $\mathrm{LPH}$, which is the cheapest $\mathrm{H}_{2}$ source thus the most interesting to exploit, with several important consumer plants pouring their excess to the LPH while simultaneously providing make-up for other (in certain cases the same) consumer plants.

\section{3- OPTIMAL REDISTRIBUTION FORMULATION}

The efficiency of $\mathrm{H}_{2}$ as a material resource is considered separately of the hydrocarbon treatment processes. Optimal management of the $\mathrm{H}_{2}$ network refers to determining the optimal production rates in the producer plants, the combination of make-up flows from each header providing $\mathrm{H}_{2}$ to each consumer plant, as well as the operating point of membrane units and other HP purges, while fulfilling all the operational constraints. Efficiency is more meaningful at the global network scope, where all the producer and consumer plants as well as the available distribution headers and connecting pipes are considered.

The optimal $\mathrm{H}_{2}$ redistribution will be solved as the mathematical solution to the non-linear problem of minimization of the operating cost subject to process constraints, according to a real-time optimization RTO approach. Initial plant state for the optimal $\mathrm{H}_{2}$ redistribution problem is the one estimated in the previous data reconciliation step.

\subsection{1- Optimal Operation Formulation: cost function}

The objective function is defined as the cost to produce $\mathrm{H}_{2}$ in the steam-reforming furnace plants; prices $\left(p_{H 4}, p_{H 3}\right)$ have been specified by Petronor ${ }^{\circledR}$ :

$$
\min _{u} J=p_{H 4} \cdot F_{H 4}+p_{H 3} \cdot F_{H 3}+f_{r H 2 H C}^{\text {weig }} \cdot \sum_{m=1}^{M} \varepsilon_{r H 2 H C, m}{ }^{2}+f_{H 2 \text { sep }}^{\text {weig }} \cdot \sum_{n=1}^{N} \varepsilon_{H 2 s e p, n}^{2}
$$




$$
\begin{array}{ll}
\text { s.t. } & f(x, u, \theta)=0 \\
& g(x, u, \theta)<0
\end{array}
$$

where $\boldsymbol{J}$ is the scalar cost function, $\boldsymbol{u}$ the available degrees of freedom, $\boldsymbol{\theta}$ model parameters, $\boldsymbol{x}$ the states, $\boldsymbol{f}$ the equality constraints corresponding to the model equations, and $\boldsymbol{g}$ the inequality constraints. The objective function also includes penalty quadratic terms for the slack variables added to guarantee feasibility in the fulfilment of the non-linear constraints, with a certain common weight for each type of constraint. The $M$ constraints for ratio $\mathrm{H}_{2} / \mathrm{HC}$ and the $\mathrm{N}$ constraints for $\mathrm{H}_{2}$ purity in the HP system are considered. Feasibility problems have not arisen in the management of linear constraints, all referring to flow rates constraints; consequently, slack variables have not been added for linear constraints with the aim of avoiding an unnecessary computational burden. The starting point for the optimal redistribution problem is the solution of the data reconciliation step, therefore linear constraints fulfilment is assured.

Only cost for the produced $\mathrm{H}_{2}$ is considered, but not the fuel gas value $\left(p_{\text {fuel }}\right)$ of the purge streams. The gap in prices is high, thus it is not expected to influence the results significantly. In any case, considering these negative prices $p_{f u e l}$ would compete against the current terms, and would result in a solution equal or even higher regarding production in steam-reforming producer plants, which is not desired. For the same reason, the cost of the by-product $\mathrm{H}_{2}$ in the catalytic reforming units is neglected, because the target is to take full advantage of it.

Since the material $\mathrm{H}_{2}$ cost is about an order of magnitude higher than the energy cost for compression according to a rough estimate with current prices (Fig. 6.1), only material costs of $\mathrm{H}_{2}$ are considered in this simplified approach.

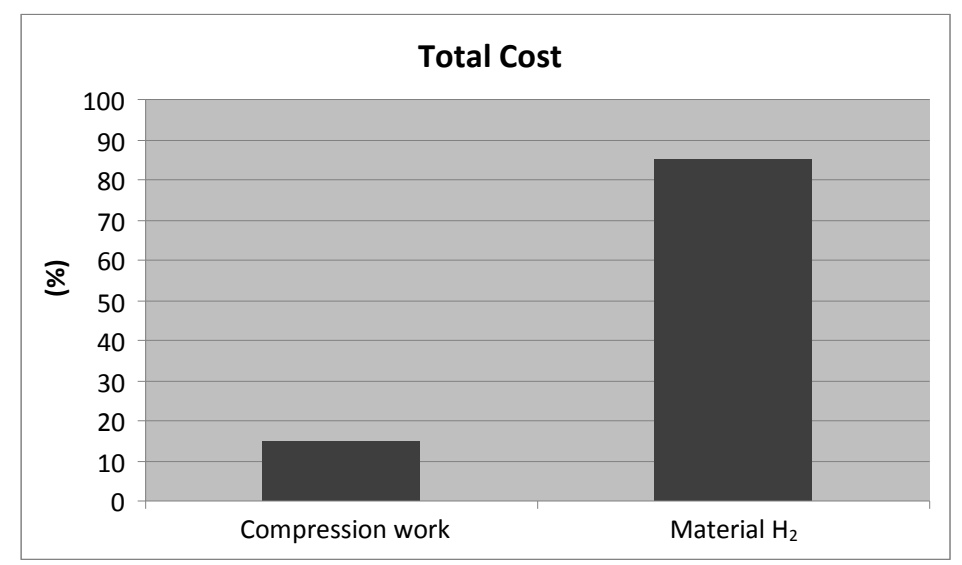

Figure 6.1. Relative estimates for material and energy costs.

The same network model as for the data reconciliation is used for the optimal redistribution problem, although with a different subset of inputs and outputs. The strategy followed is to specify or fix as invariable certain model parameters and variables from the data reconciliation 
step (where they are estimated) to the optimal redistribution step, in agreement with the following assumptions. A scheme can be seen in Figure 6.2.

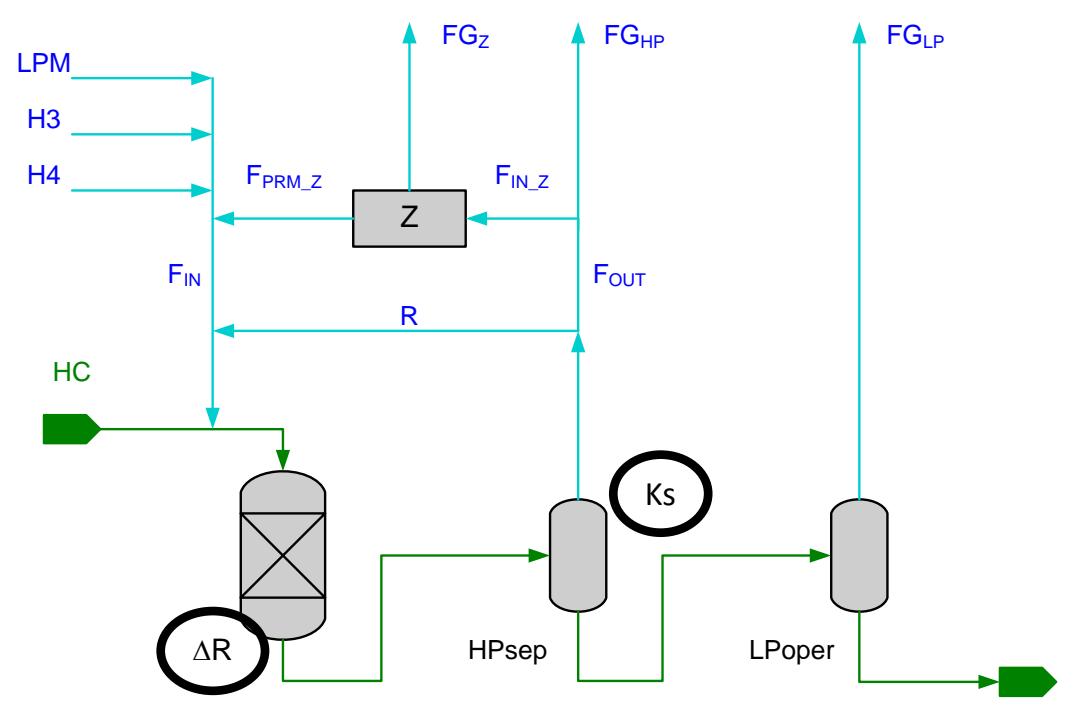

Figure 6.2. Assumptions regarding the optimal redistribution problem: invariable parameters.

The assumptions considered in the optimal $\mathrm{H}_{2}$ redistribution are:

- There are no $\mathrm{H}_{2}$ or light gases dissolved in the hydrocarbon plant inlet and outlet streams, according to process conditions;

- The simplified solubility model aforementioned is used to compute the $\mathrm{H}_{2}$ and light gases dissolved in the liquid stream from the HP/MP separators, with equal values for the parameters $\left(k_{\text {gasHC }}^{S}, k_{\alpha H 2 L G}^{S}, k_{w L I G}^{S}\right)$ as those estimated in the data reconciliation. It is equivalent to consider that the hydrocarbon liquid stream is saturated in total dissolved gases, implying constant operation regarding: i) temperature $\mathrm{T}_{\mathrm{HP} / \mathrm{MPsep}}$, controlled in the minimum value; ii) pressure $\mathrm{P}_{\mathrm{HP} / \mathrm{MPsep}}$, regulated at a fixed value; iii) hydrocarbon composition and flow rate.

Since $\mathrm{H}_{2}$ purity at the HP separator gas outlet is usually controlled at the specification, being active constraint, there will no influence in the solubility equilibrium of a change in composition due to $\mathrm{H}_{2}$ redistribution. The effect of a different composition of light ends over the $\mathrm{H}_{2}$ distribution between phases has been neglected, and is a phenomenon not modelled with the solubility equilibrium proposed, already justified.

- Consumption/generation terms for gas phase in the reactor are assumed invariable: $\Delta R_{\mathrm{H} 2}{ }^{s p}\left(\mathrm{Nm}^{3} \mathrm{H}_{2} / \mathrm{m}^{3} \mathrm{HC}\right), \Delta R_{L I G}{ }^{s p}\left(\mathrm{Nm}^{3} \mathrm{LIG} / \mathrm{m}^{3} \mathrm{HC}\right), \Delta R_{w L I G}(\mathrm{~kg} \mathrm{LIG} / \mathrm{kmol}$ LIG). It is equivalent to consider constant operation in the reactor: i) equal hydrocarbon quality (operating mode: light cyclic oil LCO, light diesel LD, heavy diesel HD, visbreaker diesel VBGO, any mix, etc.); ii) equal hydrocarbon quantity (residence time in reactor); iii) equal severity (temperature in reactor); iv) zero 
pseudo-order kinetic with respect to $\mathrm{H}_{2}$, being non-limiting reactant in enough excess, with the reaction rate therefore independent of $\mathrm{H}_{2}$ concentration and thus of $\mathrm{H}_{2}$ redistribution. This assumption is justified according to the literature.

Another important hypothesis in the optimal redistribution is that the network operation regarding connections and topological decisions is maintained, i.e., boolean decision variables are not considered in the optimization. Three different cases must be taken into account, explained next.

In the case of two-inlets excluding valves " $v 3 v \_2 i n$ " and two-outlets excluding valves "v3v_2out", both valve states should be explored in order to ascertain the optimum operating point, with an exponential increase of scenarios due to the combinatorial nature of the problem. The assumption has been made that valve states remain unchanged as in the reconciliation step. This hypothesis is not a restrictive one, as flexibility concerning these three-way valves only affects three medium-size consumer plants regarding the suppy from either one catalytic-reforming plant or the other, and has to be manually actuated in field, therefore frequent changes are neither desired not needed in general due to the similar characteristic of the make-up in both cases.

Inlet-outlet excluding valves " $v 3 S R$ " are not truly decision variables, since its opening is determined under pressure control according to hydrocarbon solubility requirements, therefore it is correct to maintain the same state as the one fixed in the data reconciliation.

In the case of elements in shared connections or headers of the type "dtOdir", whose purpose is to force zero flow rate or not irrespective of the flow direction, and which only influence one stream unlike abovementioned valves, the "open" state is considered always to solve the optimal redistribution problem thus allowing the two flow directions, as the valve state "closed" is just a particular case of the former with zero flow rate. No boolean variable is needed, and the model is continuous regarding flow rates being both positive and negative values feasible, although a small discontinuity regarding intensive variables $\left(y^{H 2}, w^{L / G}\right)$ arises when a change in the flow direction occurs. Nevertheless, the discontinuity influence over the material balances will be affected by a close to zero flow rate, and it has not proved detrimental to the optimization performance.

\subsection{2- Optimal Operation Formulation: Decision variables and Constraints}

Model $\boldsymbol{f}(\boldsymbol{x}, \boldsymbol{u}, \boldsymbol{\theta})=0$ comprises equations stated in Chapter . For simulation in EcosimPro ${ }^{\circledR}$, a number of variables equal to the independent degrees of freedom of the system need to be specified as boundaries, and the remaining model variables are computed from the system modelling equations. Specification of boundaries has been made according to physical causality, following a direction from upstream to downstream. Degrees of freedom or boundaries $(\boldsymbol{u}, \boldsymbol{\theta})$ for the optimal redistribution problem are listed in Table 6.1; they are equal to those degrees of freedom of the model, excluding variables characterizing hydrocarbon 
liquid streams and those variables and parameters fixed as "invariables" from the data reconciliation, i.e. the number of degrees of freedom is equal to that of the data reconciliation minus variables characterized as "invariable".

Variables fixed as "invariable" are listed below, corresponding to either estimated model parameters or flow rates with no influence over the $\mathrm{H}_{2}$ efficiency and whose value is fixed according to other operational criteria different from $\mathrm{H}_{2}$ use (Fig. 6.2):

- Quench flow rates for reactor bed temperature control, having no influence over $\mathrm{H}_{2}$ use;

- Bypass streams flow rates, because no additional degree of freedom is added. The other stream flow rate, the main branch not the bypass, remains as degree of freedom if appropriate;

- Recycle flow rates, again having no influence over $\mathrm{H}_{2}$ use or $\mathrm{H}_{2}$ purity in the HP system. Its value depends either on a fixed capacity compressor or on the equilibrium established between compressor head and pressure drop across the reactor closed loop. In certain cases, its value is controlled to fulfil the minimum ratio $\mathrm{H}_{2} / \mathrm{HC}$, being recycle flow rate usually four to five times that of make-up.

If the recycle compressor is centrifugal, the recycle flow rate will not remain exactly constant due to the pressure-flow new equilibrium established after the $\mathrm{H}_{2}$ redistribution. Nevertheless, as pressure drop is mainly due to liquid hydrocarbon and recycle flow rate (much higher than the make-up one), a change in the makeup flow rate will not have a significant effect on the recycle flow rate. In any case, the assumption is correct as recycle flow rate has no influence over $\mathrm{H}_{2}$ purity in the HP system.

- Streams separated in splitters after the MP/LP separation processes, that is, downstream the first HP separator. The reason is that gas flow rates separated in MP/LP systems are not freely manipulated variables but go under pressure control, therefore it does not make sense to add a degree of freedom downstream due to a splitter. Typically these are streams with valves usually closed, corresponding to reliefs to FG/flare headers activated as secondary branches in split-range pressure control structures;

- $\quad \mathrm{H}_{2}$ purity and light ends molecular weight for producer plants: $y_{i}^{H 2}, w_{i}^{L G G}$. For the catalytic-reforming plants, the same is achieved by fixing the reconciled values for the reactor and separators parameters;

- The three reactor parameters for consumption/generation: $\Delta R_{H 2}{ }^{s p}, \Delta R_{L I G}{ }^{s p}, \Delta R_{w L G G}$; for every consumer plant and also for the catalytic-reforming producer plants. It entails that the operating conditions in the reactors remain unchanged equal to the state estimated in the data reconciliation;

- The three parameters for the solubility equilibrium in separators: $k_{\text {gasHC, }}^{S} k_{\text {aH2LIG, }}^{S}$ $k_{\text {wLIG; }}^{S}$ for every consumer plant and also for the catalytic-reforming producer 
plants. It involves that the operating conditions in the separators remain unchanged equal to the state estimated in the data reconciliation.

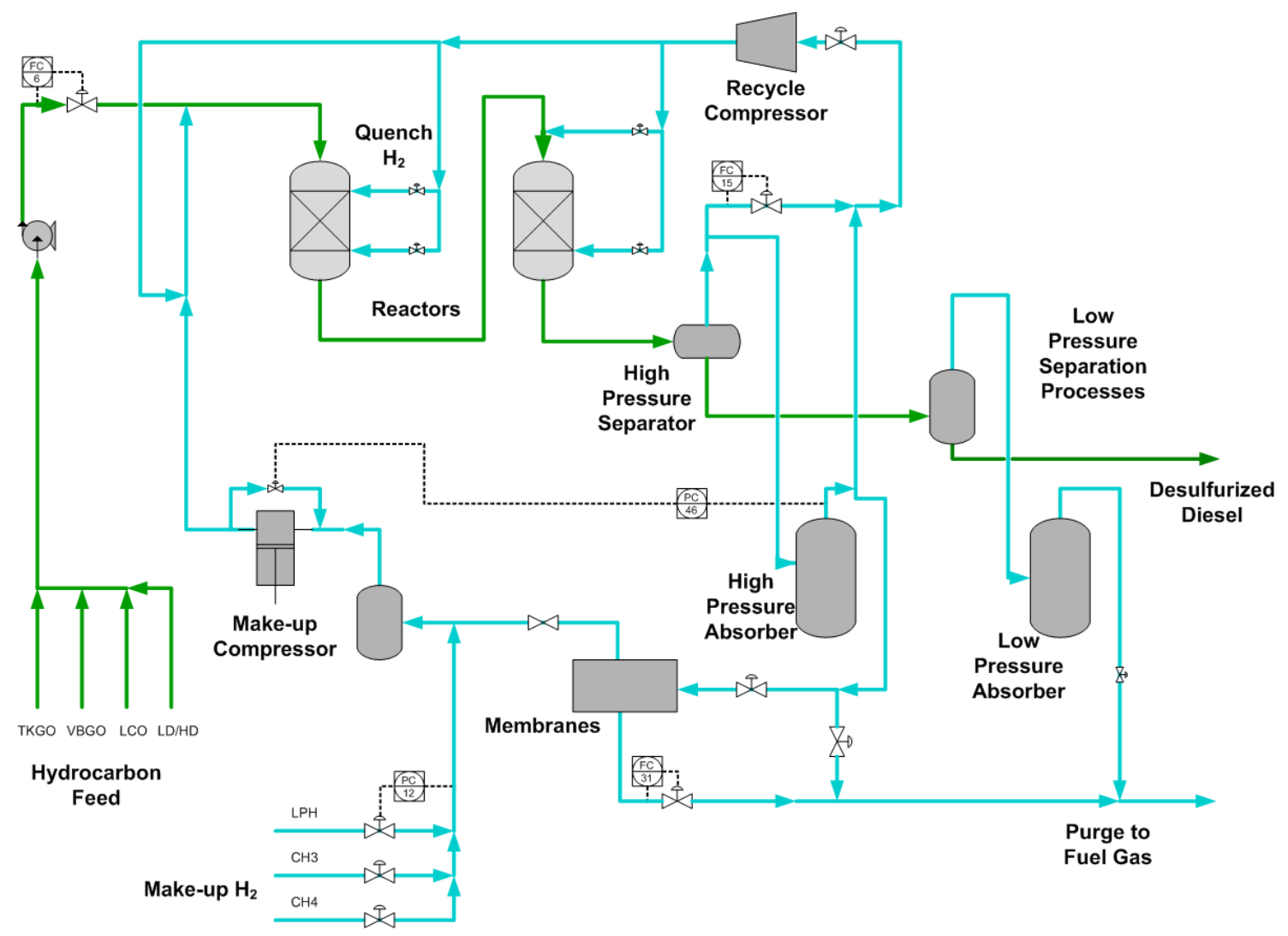

Figure 6.3. Simplified process flow diagram for a diesel hydrodesulfurizer plant with membranes.

Consequently, variables subject to change due to the optimal redistribution are the below listed flow rates; not all of them are manipulated variables in real operation, because they are interrelated with material balances (pressure control); equally, not all of them are boundaries for the EcosimPro ${ }^{\circledR}$ model. Nevertheless, there is a univocal correspondence between the decision variables for the optimal redistribution mathematical problem and the available manipulated variables in the real operation, due to model coherence and consistency with physical causality.

- Flow rates for total production in steam-reforming furnaces producer plants;

- Make-up flow rates to consumer plants from headers;

- Outlet gas flow rates from the HP separator, with all the subsequent branches and splitters; the purge from the HP system is included here. It applies for every consumer plant and also for the catalytic-reforming producer plants;

- All flow rates linked to membrane units, that is, the inlet, permeate and purge streams. 
In the selection of boundaries (independent inputs for the simulation) for gas flow rates, priority has not been given to measured streams as in the data reconciliation problem, because an initial value from the reconciled solution is already available for all model variables, but to those streams not being decision variables for the optimal redistribution problem characterized as "invariable": their value is thus easily fixed without the need of any constraint. The "invariable" set is a subset of the set of boundaries.

\begin{tabular}{llcc}
\hline Variable & Description & Units & Number \\
\hline$F \quad \begin{array}{c}\text { Volumetric flow rate at Normal conditions } \\
\text { for gas stream (either directly or } \\
\text { through ForceFbnd in valves) }\end{array}$ & $\mathrm{Nm}^{3} / \mathrm{h}$ & 48 \\
$\varepsilon_{\text {rH2HC }}$ & $\begin{array}{c}\text { Slack variable for the constraint minimum } \\
\text { ratio } \mathrm{H}_{2} / \mathrm{HC} \text { at reactor inlet }\end{array}$ & $\mathrm{Nm}^{3} \mathrm{H}_{2} / \mathrm{m}^{3}$ & 14 \\
$\varepsilon_{\text {H2sep }}$ & $\begin{array}{c}\text { Slack variable for the constraint minimum } \\
\mathrm{H}_{2} \text { purity at HP separator outlet }\end{array}$ & $\% 1 \mathrm{~mol}$ & 15 \\
& & & \\
\hline
\end{tabular}

Table 6.1. Decision variables for the optimal $\mathrm{H}_{2}$ redistribution problem.

Constraints are summarized in Table 6.2, and explained below.

- Recycle $\mathrm{H}_{2}$ purity.

One of the most important and determining constraints is to ensure a minimum $\mathrm{H}_{2}$ purity in the recycle stream or in the HP separator gas outlet, for catalyst maintenance reasons and prevention of coke build-up on the catalyst, as well as for prevention of the formation of undesired compounds or tar.

- $\mathrm{H}_{2} /$ hydrocarbon ratio at reactor inlet.

The constraint is specified in order to prevent deposition of coke over catalyst particles, ensuring enough excess of $\mathrm{H}_{2}$. The minimum ratio $\mathrm{H}_{2} / \mathrm{HC}$ is usually achieved by means of a high recycle flow rate, being typically four to five times that of the make-up flow rate.

The number of constraints for minimum HP system $\mathrm{H}_{2}$ purity and minimum $\mathrm{H}_{2} / \mathrm{HC}$ ratio is not 16 (total number of consumer plants plus catalytic-reforming units) because for certain plants only one of the two constraints has to be applied. Both are usually specified, or at least one of them.

- Steam-reforming furnace producer plants capacity.

- Membranes operating range and capacity.

The ratio of the purge to inlet flow rates $F_{P R G_{-} G} / F_{I N}$ is bounded according to historical data. The non-linear constraint resulting from the upper and lower 
bounds for this operating range is transformed into two linear ones, with infinite upper limit.

- Reciprocating compressors capacity.

It is especially important for the make-up reciprocating compressors, since they can be a limiting factor in certain plants.

- Capacity upper limit for pipes.

Although momentum balances have not been modelled, feasibility is guaranteed since no forbidden operations are allowed. In this regard, valves with mutually exclusive inlets or outlets are included, and capacity limits for pipes and compressors according to historical data are considered. This capacity constraint is especially important for the HP purges.

- Minimum Low Purity Header LPH purge to FG.

A minimum flow rate has to be allowed for because of controllability reasons and disturbance rejection, acting as a buffer against lack of $\mathrm{H}_{2}$ due to process variability.

- Explicit gas flow rates.

Constraints for explicitly calculated gas flow rates are added to assure a positive value. No constraints are added for streams modelled with "h2dir" ports, as their purpose it to model both positive and negative flow rates. In an analogous manner to that of data reconciliation, certain constraints to assure a positive flow rate are removed since they are redundant necessarily with those corresponding to other boundary or explicit streams: i) inlet flow rate to valves "dtOh2"; ii) inlet flow rate to splitter components; iii) outlet flow rate of mixer components; iv) for valve components " $v 3 v_{2} 2 i n$ " and " $v 3 v_{2}$ 2out", flow rates for the two corresponding mutually exclusive streams; v) all gas flow rates for mixtures of gas and liquid hydrocarbon, being the gas either totally solved or constituting a twophase mixture.

Problem dimension is shown in Table 6.3. The number of model equations is 14277 , and the Jacobian size $103 \cdot 103$ due to algebraic variables linked to non-linear boxes.

Optimization Problems

\begin{tabular}{lcc}
\hline no Independent Degrees of Freedom & 263 & 77 \\
no Total Independent Constraints & 188 & 149 \\
no Independent NL-Constraints & 14 & 29 \\
\hline
\end{tabular}

Table 6.3. Dimension for optimization problems: $\mathrm{n}$ - independent dof/constraints. 


\begin{tabular}{|c|c|c|c|}
\hline Constraint & Description & Units & № \\
\hline $\begin{array}{l}\text { Recycle } \mathrm{H}_{2} \text { purity, or } \\
\text { HP separator gas } \\
\text { outlet } \mathrm{H}_{2} \text { purity }\end{array}$ & $\begin{array}{l}y_{R E C, i}^{\text {Min }}-\varepsilon_{H 2 s e p, i} \leq y_{R E C, i} \leq y_{R E C, i}{ }^{M a x}+\varepsilon_{H 2 s e p, i} \\
y_{R E C}{ }^{\text {Min }}: \text { catalyst maintenance } \\
y_{R E C}{ }^{\text {Max }}: \text { surge centrifugal compressor }\end{array}$ & $\% 1 \mathrm{~mol}$ & 15 \\
\hline $\begin{array}{l}\mathrm{H}_{2} / \mathrm{HC} \text { ratio at reactor } \\
\text { inlet }\end{array}$ & $\left(F_{i} / F_{H C, j}\right)^{M i n} \leq F_{i} / F_{H C, j}+\varepsilon_{r H 2 H C, i}$ & $\begin{array}{l}\mathrm{Nm}^{3} \mathrm{H}_{2} \\
/ \mathrm{m}^{3} \mathrm{HC}\end{array}$ & 14 \\
\hline $\begin{array}{l}\text { Producer plants } \\
\text { capacity }\end{array}$ & $\left(F_{i}\right)^{\operatorname{Min}} \leq F_{i} \leq\left(F_{i}\right)^{\operatorname{Max}}$ & $\mathrm{Nm}^{3} / \mathrm{h}$ & 2 \\
\hline $\begin{array}{l}\text { Membranes } \\
\text { operating range and } \\
\text { capacity }\end{array}$ & $\begin{array}{l}\left(F_{F G_{Z} Z} / F_{I N_{Z} Z}\right)^{\operatorname{Min}} \leq F_{F G_{-} z} / F_{I N_{-} Z} \leq\left(F_{F G_{-} Z} / F_{I N_{-} z}\right)^{\operatorname{Max}} \\
\left(F_{I N_{-} z}\right)^{\operatorname{Min}}<F_{I N_{-} z}<\left(F_{I N_{-}} z\right)^{\operatorname{Max}}\end{array}$ & $\mathrm{Nm}^{-} / \mathrm{h}$ & 6 \\
\hline $\begin{array}{l}\text { Make-up } \\
\text { Reciprocating } \\
\text { Compressors } \\
\text { capacity }\end{array}$ & $F_{i} \leq\left(F_{i}\right)^{\operatorname{Max}}$ & $\mathrm{Nm}^{3} / \mathrm{h}$ & \\
\hline $\begin{array}{l}\text { Capacity upper limit } \\
\text { for pipes, specially } \\
\text { HP purges }\end{array}$ & $F_{i} \leq\left(F_{i}\right)^{\operatorname{Max}}$ & $\mathrm{Nm}^{3} / \mathrm{h}$ & \\
\hline $\begin{array}{l}\text { Low Purity Header } \\
\text { LPH purge to FG - } \\
\text { Minimum flow } \\
\text { Controllability }\end{array}$ & $\left(F_{L P H}\right)^{\mathrm{Min}} \leq F_{L P H}$ & $\mathrm{Nm}^{3} / \mathrm{h}$ & 1 \\
\hline $\begin{array}{l}\text { Explicit gas flow } \\
\text { rates }\end{array}$ & $0 \leq F_{i} \leq F_{\infty}$ & $\mathrm{Nm}^{3} / \mathrm{h}$ & \\
\hline
\end{tabular}

Table 6.2. Constraints for the optimal $\mathrm{H}_{2}$ redistribution problem.

\section{Limits and Initialization}

Limits and initialization values are extremely important to solve the optimization problem. Initialization values for all variables are those corresponding to the previously solved data reconciliation problem. In the optimal redistribution problem, lower/upper bounds for all decision variables and constraints are constant and equal irrespective of the experiment, except for the following cases: 
- Constraints regarding minimum $\mathrm{H}_{2}$ purity in the HP system vary according to the particular experiment, since specification is forced to be equal to the value read in operation. In this way, the potential profit margin in optimal redistribution is really achieved due to gas redistribution and not as a consequence of a less demanding constraint, or specification change. Alternatively, although not used here, a fix constant value can be specified for all experiments;

- Equally, the minimum purge flow rate from the LPH to the FG header is also forced to be equal to the value read in operation, so that savings corresponding to the optimal solution found are not influenced by a change in the specification. Alternatively, although not used here, a fix constant value can be specified for all experiments;

- $\quad$ Since it depends on the hydrodesulfurizer operating mode, the minimum ratio $\mathrm{H}_{2} / \mathrm{HC}$ at reactor inlet is either read from an existing tag in the SCADA system or is either forced to the reconciled value for those consumer plants without the corresponding tag;

- In order to account for special operational criteria according to which constraints are deliberately not fulfilled in operation, e.g. at the end of the catalyst cycle, the less restrictive value between the reconciled process value and the fixed specification is automatically chosen. This applies to both the operating ranges in membranes and the ratio $\mathrm{H}_{2} / \mathrm{HC}$.

As aforementioned, appropriate limits for both decision and constraints variables are extremely important; too broad limits can lead to suboptimal solutions because the probability of local optima increases, whereas too narrow ones (even for a particular variable) can lead also to bad results due to lack of flexibility in other related variables along the steps of the search. The particular trade-off depends on the variable and the problem structure.

\section{4- OPTIMAL REDISTRIBUTION IMPLEMENTATION}

Implementation has been performed in the EcosimPro ${ }^{\circledR}$ environment, with a SNOPT ${ }^{\circledR}$ SQP algorithm as optimization solver, following a direct sequential approach (Figure 6.4). The SQP routine was chosen according to the characteristics of the problem being solved, non-linear and with a medium number of decision variables and constraints; being the solver deterministic, a solution is obtained in a reasonable computation time. Another implementation is also available in $\mathrm{GAMS}^{\circledR}$ modelling and optimization environment (G. Gutiérrez, D. Sarabia et al.), following a simultaneous approach and using the same SNOPT ${ }^{\circledR}$ SQP solver. Whereas in EcosimPro ${ }^{\circledR}$ execution times for the optimal redistribution problem range from 1 to 3 hours in an Intel ${ }^{\circledR}$ Core $^{\mathrm{TM}} \mathrm{i} 7,2.80 \mathrm{GHz}, 4.00 \mathrm{~GB}$ RAM, being the execution time to run a steady state of 1 second approximately, in GAMS ${ }^{\circledR}$ execution times are in the 
order of seconds, with about 5 additional minutes for importing results in the developed Excel ${ }^{\circledR}$ user interface.

The linear constraint treatment and the two-stage procedure for the Snopt ${ }^{\circledR}$ call are analogous to that explained in the corresponding epigraph for Data Reconciliation in Chapter 5.

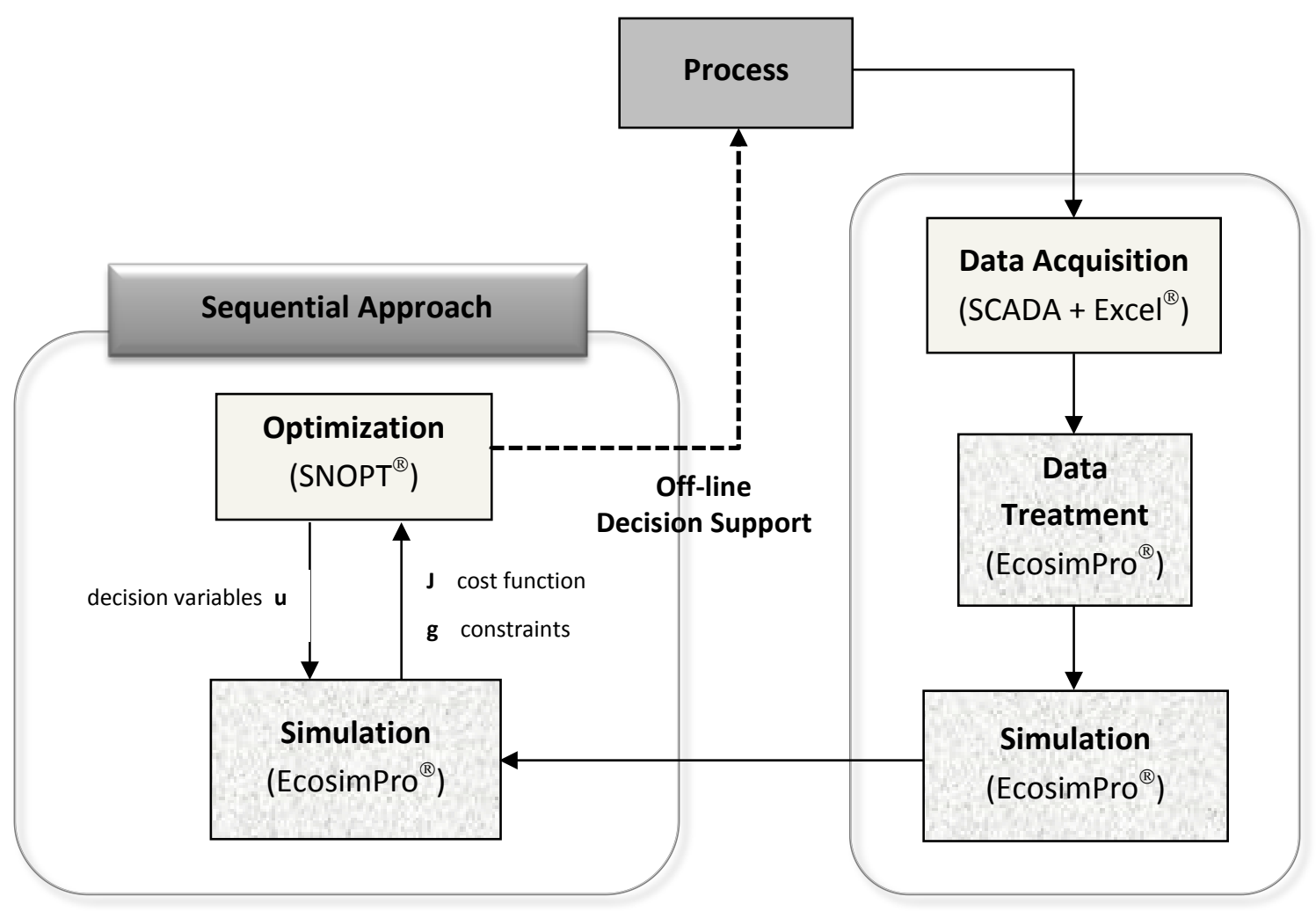

Figure 6.4. Scheme for the optimal $\mathrm{H}_{2}$ redistribution implementation in EcosimPro ${ }^{\circledR}$.

Scale factors for all decision and constraint variables were automatically assigned depending on the values at the initial state. Again, sometimes better results were achieved by changing in one order of magnitude the scale factor affecting the objective function or certain important variables. Experience has shown that an adequate tuning of scale factors determines to a great extent the solution achieved with the SNOPT ${ }^{\circledR}$ algorithm, may be in this case where EcosimPro $^{\circledR}$ does not provide automatic derivatives for model variables, coupled with a problem where flow rates can vary in a great extent between zero and a certain value or vice versa from the initial state to the optimum, as opposed to data reconciliation where the optimum is always close to raw measurements, thus with narrower ranges for all measured flow rates. 


\section{5- OPTIMAL OPERATION SOLUTION ANALYSIS}

In the off-line analysis performed for 25 scenarios covering a broad range in operating conditions along eighteen months, all solutions fit to the logical pattern explained below. For the sake of comprehension, first the general framework for an improved efficiency in $\mathrm{H}_{2}$ use will be described, and then solutions and optimal strategies analysed.

\subsection{1- Description of the general framework for optimal management}

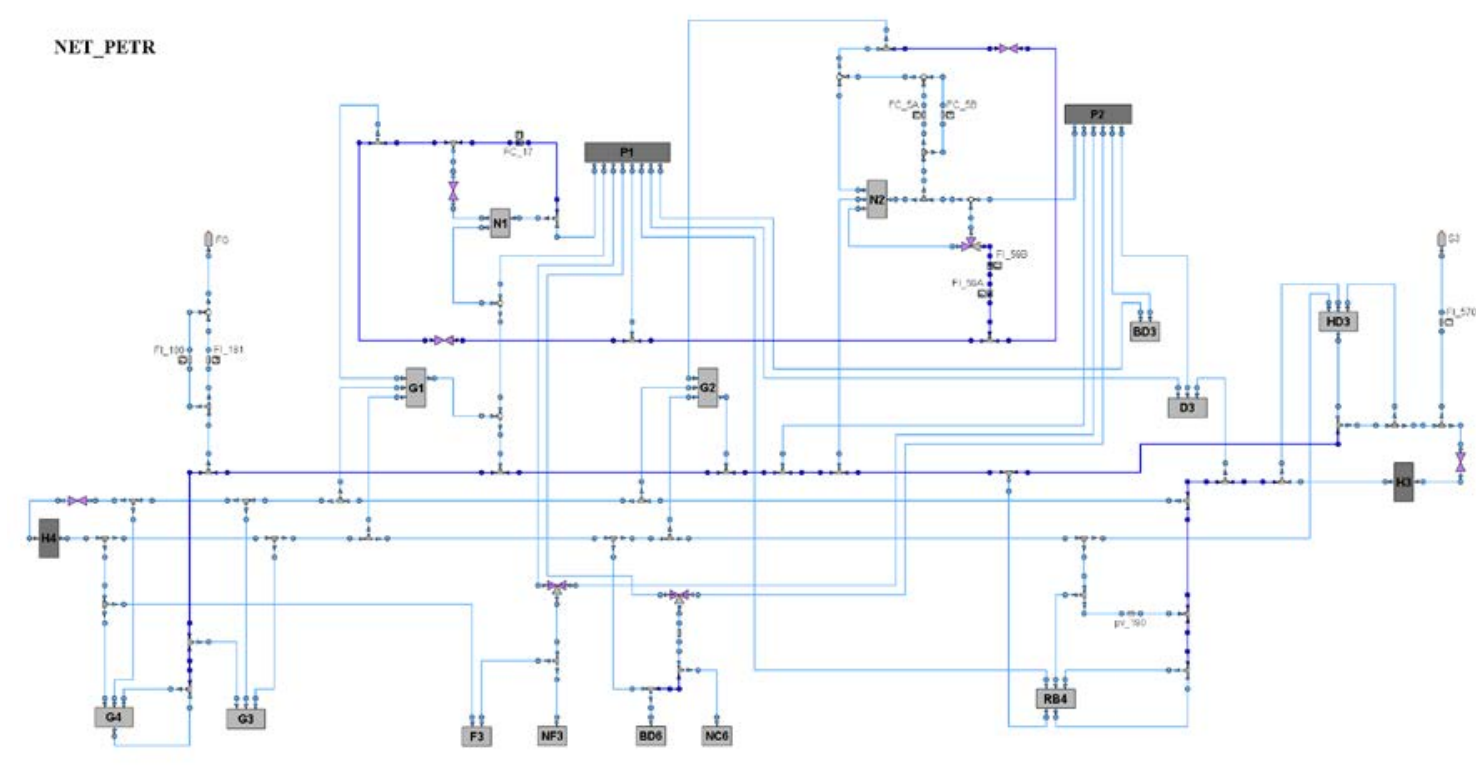

Fig. 6.5. Schematic of the Petronor ${ }^{\circledR}$ refinery $\mathrm{H}_{2}$ network.

At the global network scope, two extreme scenarios can be distinguished according to the availability of $\mathrm{H}_{2}$ in the Low-Purity Header LPH, i.e. low-purity $\mathrm{H}_{2}$ in excess either from consumer plants HP purges or from catalytic-reforming units, and which can be further reused (dark blue header in Fig. 6.5 with two direct purges to FG). These two extreme cases are excess of $\mathrm{LPH} \mathrm{H} \mathrm{H}_{2}$, when the $\mathrm{LPH} \mathrm{H}_{2}$ needs to be purged because it is not useful to fulfil the constraints, mainly the specification for the HP system purity ${ }_{\mathrm{HP}_{P}}{ }^{\mathrm{H}}$; and deficit of $\mathrm{LPH} \mathrm{H}_{2}$, when all of it can be reused as make-up thus substituting high-purity $\mathrm{H}_{2}$ from the steam-reforming furnace producer plants. Scenarios found in industrial practice are in between these two, sharing characteristics from both of them. These two scenarios, deficit or excess of LPH $\mathrm{H}_{2}$, mainly depend on:

- $\mathrm{H}_{2}$ generated as by-product in the catalytic-reforming plants. $\mathrm{H}_{2}$ production in these units experiences a monthly cycle due to catalyst partial regeneration; also reactor severity influences $\mathrm{H}_{2}$ production. In the long term, catalyst activity 
decreases progressively until completely removed every twenty months approximately;

- Minimum $\mathrm{H}_{2}$ purity specifications $\mathrm{Y}_{\mathrm{HPsep}}{ }^{\mathrm{H} 2}$ in the high pressure separator gas outlet $\left(H P_{\text {SEP }}\right)$ at consumer plants. Regarding the use of $\mathrm{H}_{2}$ from the $L P H$, these specifications are determining: low specifications enable to take advantage of all the low-purity $\mathrm{H}_{2}$ in excess, whereas higher specifications imply the need to purge at the network level, get rid of low-purity $\mathrm{H}_{2}$ in excess because it is no longer useful to fulfil the constraints.

Network states and characteristics for these two extreme cases are resumed in Table 6.4, and briefly explained next. When $y_{\text {HPsep }}{ }^{H 2}$ specifications are high, there is excess of LPH $\mathrm{H}_{2}$ that needs to be purged, because not all of it can be used to fulfil the constraints. The specification $y_{\text {HPsep }}{ }^{H 2}$ is binding constraint in all consumer plants, because the excess of $\mathrm{LPH} \mathrm{H}_{2}$ enables to adjust its value; in the deficit case, $\mathrm{H}_{2}$ requirements in consumer plants have to be satisfied with high-purity $\mathrm{H}_{2}$ from steam-reforming furnaces units, and as a result a gap can arise in the $y_{\text {HPsep }}{ }^{H 2}$ with respect to the minimum specified. High-pressure HP purges in consumer plants going directly to $F G$ instead of to other headers are not minimized in case of excess, which entails maximizing make-up from the catalytic-reforming plants. The membranes are a particular case of HP purges to FG, and consequently they are needed in scenarios of LPH $\mathrm{H}_{2}$ excess. For each of the previous items, the opposite applies in the extreme case with deficit of $\mathrm{LPH} \mathrm{H}$.

\begin{tabular}{|c|c|c|}
\hline SCENARIO: $\mathrm{H}_{2}$ AVAILABILITY IN LPH & EXCESS & DEFICIT \\
\hline $\begin{array}{l}\text { - [High/Low] specifications for minimum } \boldsymbol{y}_{H P s e p}{ }^{H 2} \\
\mathrm{H}_{2} \text { purity in } \mathrm{HP}_{\mathrm{SEP}} \text { : [not all/all] } \mathrm{H}_{2} \text { from LPH useful }\end{array}$ & High, not all & Low, all \\
\hline $\begin{array}{l}\text { - Minimum } \boldsymbol{y}_{H P s e p}{ }^{H 2} \text { active constraint in all } \\
\text { consumer plants: [enough/not enough] } \mathrm{H}_{2} \text { from } \\
\text { LPH }\end{array}$ & Yes, enough & $\begin{array}{l}\text { Gap w.r.t. } \\
\text { minimum in } \\
\text { certain plants: } \\
\text { requirements } \\
\text { satisfied partly } \\
\text { with high-purity } \\
\text { producers }\end{array}$ \\
\hline $\begin{array}{l}\text { - Membranes in operation [yes/no]: purges } \\
\text { [needed/not needed] }\end{array}$ & Yes, needed & No, not needed \\
\hline $\begin{array}{l}\text { - Mandatory purges to FG in } \mathrm{HP}_{\mathrm{SEP}} \text { [not } \\
\text { minimized/minimized]: make-up from [Catalytic- } \\
\text { reforming/Steam-reforming furnaces] }\end{array}$ & $\begin{array}{l}\text { Not minimized, } \\
\text { Catalytic- } \\
\text { reforming }\end{array}$ & $\begin{array}{l}\text { Minimized, } \\
\text { Steam-reforming } \\
\text { furnaces }\end{array}$ \\
\hline
\end{tabular}

Table 6.4. Network state for the two extreme cases regarding low-purity $\mathrm{H}_{2}$ availability. 
Three categories can be established for consumer plants regarding the decision variable HP purge flow rate, ordered according to decreasing efficiency in $\mathrm{H}_{2}$ use:

- $\quad$ HP purge to the Low Purity Header (LPH), collecting $\mathrm{H}_{2}$ excess from different plants. The HP purge $\mathrm{H}_{2}$ purity is the same as the constraint for $\mathrm{H}_{2}$ purity in the $\mathrm{HP}_{\text {SEP }}\left(\mathrm{y}_{\text {HPsep }}{ }^{\mathrm{H}}\right)$ in all consumer plants;

- $\quad$ HP purge to the Fuel Gas Header (FG) through a membrane unit, thus recovering part of the $\mathrm{H}_{2}$ in the permeate. In this case, the purge $\mathrm{H}_{2}$ purity is lower than the constraint for $y_{\text {HPsep }}{ }^{H 2}$;

- HP purge directly to the Fuel Gas Header (FG). Again, the purge $\mathrm{H}_{2}$ purity is the same as the constraint for $\mathrm{y}_{\mathrm{HPsep}}{ }^{\mathrm{H}}$; therefore, this is the case only for plants with a low $\mathrm{H}_{2}$ purity constraint in the $\mathrm{HP}_{\text {SEP }}$, thus not being profitable the reuse of excess $\mathrm{H}_{2}$.

Network design is such that the HP purge is sent directly to FG only for medium-small consumer plants operating at a not very high $\mathrm{Y}_{\mathrm{HPsep}}{ }^{\mathrm{H}}$, whereas very intensive consumer plants, usually also with high purity $\mathrm{Y}_{H P s e p}{ }^{\mathrm{H}}{ }^{2}$ constraints, send their excess to other headers for being further reused as make-up because its recovery is worthy. The flowsheet diagram for an important hydrodesulfurization consumer plant can be seen in Fig. 6.6, with make-up from three headers, $\mathrm{H} 4 \mathrm{H}, \mathrm{H} 3 \mathrm{H}$ and $\mathrm{LPH}$, and $\mathrm{HP}$ purge to the $\mathrm{LPH}$ (through valve $F Y_{-} 18 \mathrm{~A}$ ), with a direct HP purge to FG only for security reasons under split-range pressure control (through valve FY_18B), as well as the corresponding MP and LP purges to FG (flow meters FI_15 and FI_24 respectively).

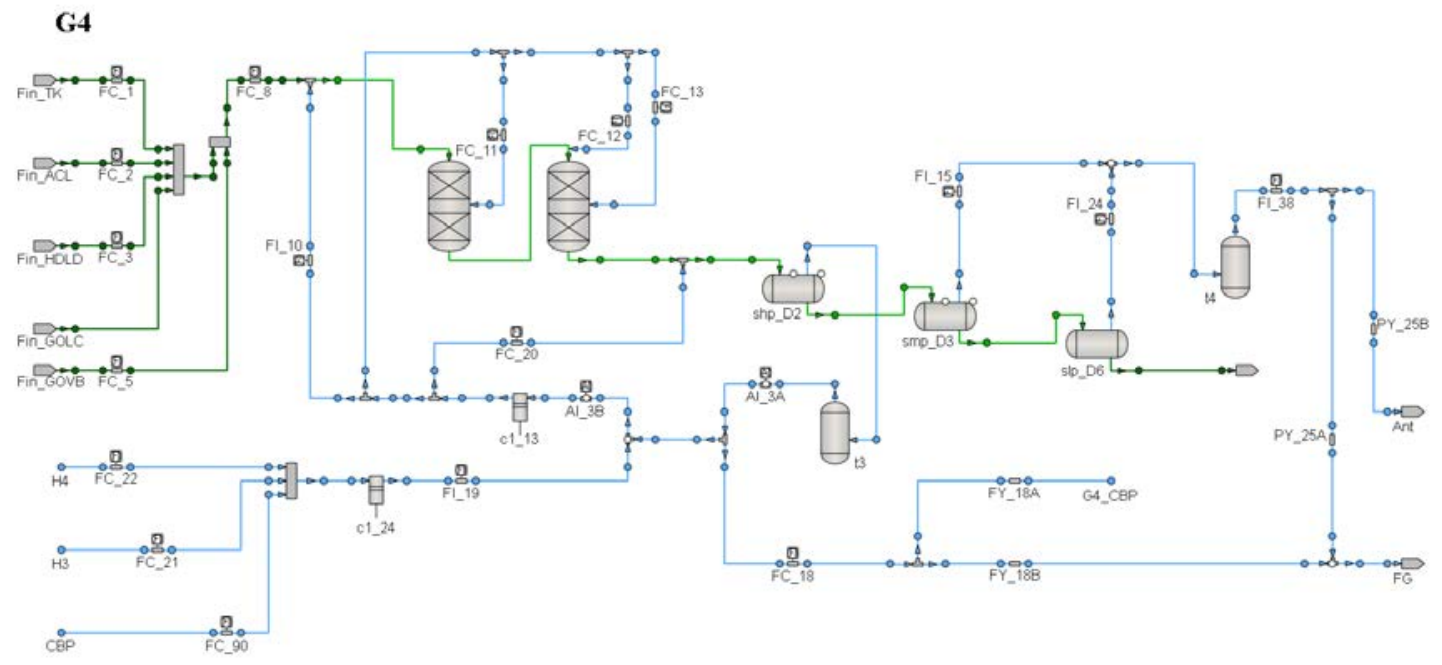

Fig. 6.6. Schematic of diesel hydrodesulfurization consumer plant. 


\subsection{2- Optimal operation solution analysis}

The solution analysis will be centred on the available decision variables for the optimal network operation. These decision variables can be organized into two groups, according to their influence or effect over efficiency in $\mathrm{H}_{2}$ use: i) HP purges from both consumer plants and from headers, enabling in the end to purge to $F G$ all the generated light ends, therefore guaranteeing the fulfilment of the minimum $\mathrm{H}_{2}$ purity constraints. The decision on the HP purge determines whether the make-up has to be done from steam-reforming furnaces, from catalytic-reforming plants, or a combination, as will be later shown; ii) make-up flow rates from the different headers to consumer plants, enabling an additional margin in the case of trade-offs or preferential make-ups from either one steam-reforming furnace plant or the other, i.e. decisions between the high-purity producer plants. The result of the analysis for the two groups of decision variables is explained below.

\section{i) HP purges}

An analysis of solutions for the optimal operation enables to identify a clear logical pattern regarding high pressure (HP) purges from consumer plants or headers. The solution is the logical one, that is, to purge Low Purity Header LPH excess (in general low-purity $\mathrm{H}_{2}$ ), if any, at the network scope through the HP purge to FG at the lowest $\mathrm{H}_{2}$ purity until it gets saturated, following an increasing order of $\mathrm{H}_{2}$ purity to purge in consumer plants.

In this sense, the optimal operation could be ascertained in advance without performing a mathematical optimization, although requiring longer times in order to reach steady operation after a sequential implementation of changes in the HP purge flow rates of consumer plants. Therefore, the tool could help to save time in the identification of suboptimal operation and implementation of the optimal policy.

In the network studied, there are certain medium-size consumer plants, as well as the membrane units, whose HP system purges operate at a lower $\mathrm{H}_{2}$ purity than the LPH one; their joint capacity is enough to process all the low-purity $\mathrm{H}_{2}$, as a result the LPH purge is always minimized in the optimal operation.

All scenarios studied correspond to cases between the aforementioned two extreme ones, deficit and excess of $\mathrm{LPH} \mathrm{H}_{2}$, for which the solutions analysed show that:

- Direct HP purges to FG in consumer plants are prioritized according to their increasing $\mathrm{H}_{2}$ purity: the plant with the lowest $\mathrm{H}_{2}$ purity in the HP system is the first to maximize its purge to FG. The purge flow rate is the maximum when the $\mathrm{H}_{2}$ purity constraint is fulfilled; further purge increase is not efficient as there will be a gap in the minimum $\mathrm{H}_{2}$ purity constraint instead of being binding constraint.

When the direct purge to FG for a plant is the maximum thus saturated, the purge in another plant starts to open. It will correspond to the plant with the following $\mathrm{H}_{2}$ purity in the HP system, in increasing order. Higher purge flow rate implies 
higher make-up from the LPH, again while guaranteeing that the $\mathrm{H}_{2}$ purity is binding constraint. For a plant with make-up from two sources, one from the LPH and the other from one steam-reforming furnace producer plant, $\mathrm{H} 3 \mathrm{H}$ or $\mathrm{H} 4 \mathrm{H}$, an increase in the LPH make-up entails a decrease in the $\mathrm{H} 3 \mathrm{H} / \mathrm{H} 4 \mathrm{H}$ make-up; that is why the purge must be increased, to ensure the minimum $\mathrm{H}_{2}$ purity constraint in spite of the exchange in the make-up flow rates. As a consequence, the low-purity $\mathrm{H}_{2}$ exploitation increases and the production of $\mathrm{H}_{2}$ in $\mathrm{H} 4 / \mathrm{H} 3$ decreases, resulting in an improved efficiency in $\mathrm{H}_{2}$ use.

- Usually, only one direct HP purge to FG for a particular consumer plant is not either at zero or either saturated (Figure 6.7). As a result, direct HP purges to FG in consumer plants are in one of the following three states: $\left[\boldsymbol{F}_{F G}=0 / \boldsymbol{F}_{F G}\right.$ increases progressively / $\boldsymbol{F}_{F G}$ saturated when maximum make-up from the $\mathrm{LPH}$ achieved, i.e. further increase means either unfeasible minimum $\mathrm{H}_{2}$ purity constraint or either a suboptimal gap with respect to the required minimum constraint].

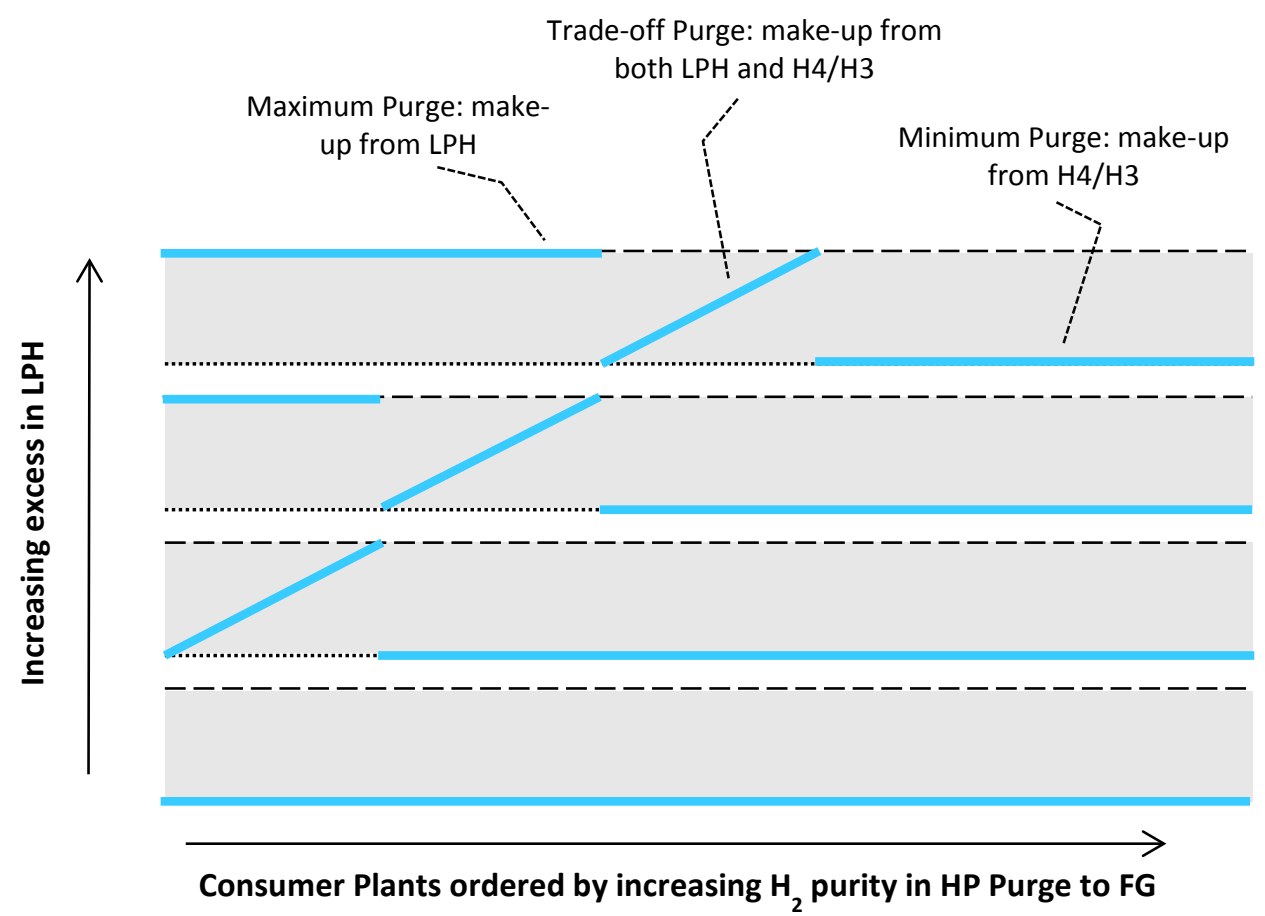

Figure 6.7. Diagram for the optimal policy regarding HP purges to FG at the network scope.

\section{ii) Make-up flow rates from the different producer plants, trade-offs}

Regarding trade-offs in the optimal $\mathrm{H}_{2}$ redistribution from the different producer plants to the different consumer plants, the margin is slightly noticeable; the difference both in $\mathrm{H}_{2}$ purity and price between the two steam-reforming furnaces producer plants $\mathrm{H} 4$ and $\mathrm{H} 3$ is very small, existing a significant gap with respect to the low-purity $\mathrm{H}_{2}$ in the LPH. That is, it is not 
important whether a consumer plant has a preferential make-up from either $\mathrm{H} 4 \mathrm{or} \mathrm{H} 3$, the key is to make the most out of the LPH hydrogen.

The biggest consumer plants are those with the highest flexibility regarding make-up, and with potential for profit increase due to make-up redistribution or exchange among the make-ups from the different headers. Provided a roughly similar distribution of $\mathrm{LPH} \mathrm{H}_{2}$ among the biggest consumer plants exists, there is no significant margin in sending the produced $\mathrm{H}_{2}$ in a certain steam-reforming furnace plant preferentially to a given consumer plant or to another, for broad ranges of flow rates. That is, provided a reasonable distribution of the available lowpurity $\mathrm{H}_{2}$ is made among the biggest consumer plants, according to their minimum $\mathrm{HP} \mathrm{H}_{2}$ purity constraints, the margin gained by exchanging the make-up flow rates by small quantities is hardly appreciable. Nevertheless, the profit margin is higher out of these ranges. The reason to provide a certain amount of $\mathrm{LPH} \mathrm{H}_{2}$ to all the big consumer plants is to avoid a suboptimal gap with respect to the required HP minimum $\mathrm{H}_{2}$ purity constraint, which implies to preferentially accomplish the saturation in gas of the HP outlet liquid with light ends instead of with high-purity $\mathrm{H}_{2}$, therefore with lower loses through the MP/LP systems downstream.

\subsection{3- Case Study Conclusions}

Regarding the use of the tool for network on-line decision-support purposes by Petronor ${ }^{\circledR}$, it can be concluded that:

- Even though the potential is not high, there is an opportunity for profit in operation due to frequent changes in scenarios and the important amounts involved, with a low margin up to $0.5-1 \%$ depending on the particular scenario considered.

A high profit margin could not be expected either, since the main degree of freedom for the $\mathrm{H}_{2}$ optimal redistribution is the HP system purge for all consumer plants together with the global network, which only accounts for about $3-5 \%$ of the total $\mathrm{H}_{2}$ production at the network level. Approximately $10 \%$ of the total $\mathrm{H}_{2}$ production corresponds to losses in the MP/LP purges due to the $\mathrm{H}_{2}$ solved downstream the HP separator, and approximately $85 \%$ corresponds to $\mathrm{H}_{2}$ consumption in chemical reactions (Fig. 6.8).

- According to the previous analysis, the most important variables to monitor with the aim of suboptimal operation identification, and subject to changes depending on the particular scenario, are the following: i) make-up from the LPH to the biggest consumer plants, just to assure a roughly even distribution; ii) direct HP purges to FG for certain medium-size consumer plants; iii) purge to FG from the membrane unit in operation, as well as the inlet flow rate, i.e. membranes optimal point of operation.

There are three membrane units in the network, although with a fix priority order. The one which is first turned on belongs to a consumer plant where the HP purge goes directly to $F G$ instead of to the $L P H$, in spite of its high $\mathrm{H}_{2}$ purity. 
Consequently, the purge in this plant is always done through the membranes unit, in order to avoid the direct purge to FG. This membrane capacity is large, as a result it is rarely saturated and other membranes are not needed because lowpurity $\mathrm{H}_{2}$ availability is not enough to increase purge requirements at the network scope.

- Despite the $\mathrm{H}_{2}$ network complexity and potential connections, especially regarding simultaneous/exclusive make-up from the two catalytic-reforming units to certain consumer plants, the degrees of freedom available are significantly reduced due to preferential paths and operational reasons, as explained in the epigraph for Network Topology in Chapter 4. Furthermore, the assumption is in accordance with the achieved results, since the potential margin regarding a preferential make-up from either one catalytic-reforming plant or the other (P1/P2) will be negligible, due to similar prices (considered free) and purities for the $\mathrm{H}_{2}$ produced in these units, and provided the set of binding constraints is not altered as a result of a different connection, for example regarding the minimum ratio $\mathrm{H}_{2} / \mathrm{HC}$ in linked plants without recycle stream.

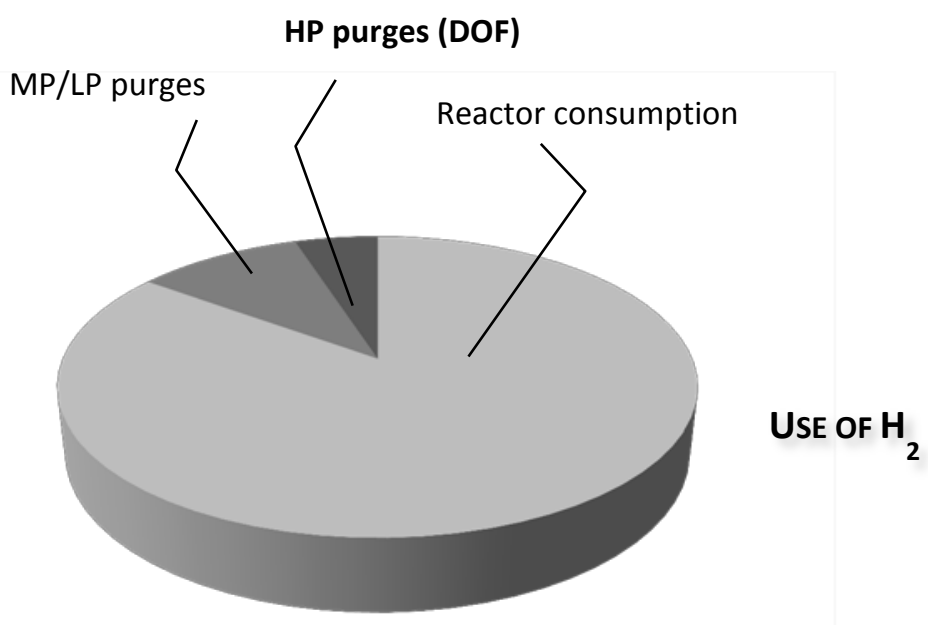

Figure 6.8. Approximate use of the produced $\mathrm{H}_{2}$ at the network scope.

\section{6- ADDITIONAL EXPLOITATION OF MODEL AND OPTIMIZATION CAPABILITIES}

The model and the optimization capabilities have been further exploited in two applications, briefly explained next. The first one is a study made in collaboration with the Repsol ${ }^{\circledR}$ Technology Center -CTR-, with the aim of assessing different changes in the topological design of the Petronor ${ }^{\circledR}$ refinery $\mathrm{H}_{2}$ network, steered towards $\mathrm{H}_{2}$ production minimization and cost reduction. The second one is a What-If simulation used to experiment the effects over $\mathrm{H}_{2}$ efficiency of different operational strategies, complemented with a set of defined resource 
efficiency indicators (REIs) intended for on-line decision support, in the framework of the European project MORE.

\subsection{1- Study on design alternatives regarding new connections}

The Repsol ${ }^{\circledR}$ Technology Center (CTR) sited in Móstoles (Madrid) leaded a cooperation to study different topologies regarding new connections of pipes in the Petronor ${ }^{\circledR}$ hydrogen network; the cooperation lasted for several months and finished in July 2014. Three alternatives where studied in detail, as well as their impact on efficiency by comparing $\mathrm{H}_{2}$ production requirements in each case. In the first place, the CTR team proposed a base case scenario representative of the expected average case for the following year, in which $\mathrm{H}_{2}$ consumption in reactors and hydrocarbon processed were completely specified. For this base case the network operation was optimized, employing the calibrated model and the optimal redistribution problem implemented in EcosimPro ${ }^{\circledR}$ and using SNOPT ${ }^{\circledR}$ routine as solver.

The three alternatives studied are:

i) The gas outlet of the medium-pressure MP separators for G1 and G4, the two plants operating at the highest $\mathrm{H}_{2}$ purity in the HP separators being also intensive consumer plants, are connected to a membranes unit usually not switched on. These are the two purges to Fuel Gas of higher $\mathrm{H}_{2}$ purity and flow rate in the network, therefore those whose recovery has the highest priority. The consumer plant to which the membranes belong does not require a high constraint of $\mathrm{H}_{2}$ purity in the HP separator $\mathrm{y}_{\text {HPsep }}{ }^{\mathrm{H}}$, thus it is feasible to recycle for make-up low purity $\mathrm{H}_{2}$ from its membrane unit. It is interesting to remark that the membranes permeate $\mathrm{H}_{2}$ purity is high when the inlet corresponds to a HP stream at a certain high purity verifying the corresponding constraint for minimum ${ }_{\text {HPSep }^{H 2}}{ }^{\mathrm{H}}$; however for all these alternatives regarding the recovery of low purity $\mathrm{H}_{2}$ from a MP purge currently sent to $\mathrm{FG}$, the permeate $\mathrm{H}_{2}$ purity is significantly lower as compared with the typical membranes operation.

ii) The gas outlet of the MP separators for G1, G4 and HD3, the three plants operating at the highest $\mathrm{H}_{2}$ purity in the HP separators being also intensive consumer plants, are connected to a membranes unit usually not switched on, the same as in i). These are the three purges to Fuel Gas of higher $\mathrm{H}_{2}$ purity and flow rate in the network, therefore those whose recovery has the highest priority.

iii) In consumer plant G4, the MP separator purge is recycled directly to the make-up. The connection is internal to the plant, in a straightforward way, and without the use of membranes to increase its purity. The rest of the make-up sources for G4 are highpurity $\mathrm{H}_{2}$ from $\mathrm{H} 4 / \mathrm{H} 3$ headers. Notice that it would probably not be feasible an analogous structure for $G 1$, as it is mandatory that $G 1$ is supplied with $H_{2}$ from the catalytic-reforming P1 (sent across a naphtha desulfurization plant N1 without HP absorber, and constituting the HP purge for N1), of lower purity than the $\mathrm{H}_{2}$ from the steam-reforming furnaces producer plants $\mathrm{H} 4 / \mathrm{H} 3$. 
For each alternative, the optimal redistribution problem was solved, and the savings in $\mathrm{H}_{2}$ production were analysed. It was concluded that recovering MP purges of low purity was not interesting in the scenario considered, because there was already excess of low purity $\mathrm{H}_{2}$ in the $\mathrm{LPH}$, not useful to fulfil the high $\mathrm{H}_{2}$ purity constraints. As a result of recycling MP purges, both membranes units were operating near to their capacity upper bound with a not significant reduction in the $\mathrm{H}_{2}$ total production in steam-reforming furnace producer plants. A broader range of scenarios should also be considered for a more substantial study.

\subsection{2- What-If analysis}

Additionally, the problem of the $\mathrm{H}_{2}$ network optimal management -comprising the model, simulation and optimizations implementations- is being used as case study in the European project MORE "Real-time Monitoring and Optimization of Resource Efficiency in Integrated Processing Plants", from November 2013 until November 2016, intended for the real-time monitoring of the efficiency in the use of material and energy resources.

A demo based on the EcosimPro ${ }^{\circledR}$ simulation for the Petronor ${ }^{\circledR}$ hydrogen network has been partially configured in the EDUSCA ${ }^{\circledR}$ SCADA, developed by Ph.D. Raúl Alves Santos in the Dpt. of Systems Engineering and Automation a few years ago. The SCADA interface is friendly from a user's point of view. A What-If analysis is therefore available in simulation, where all process inputs can be manipulated; both inputs and outputs correspond exactly to those physically meaningful in real operation. The model begins the simulation from a snapshot based on realtime data, although the value of all inputs and parameters can be modified later. The tool (Fig. 6.9) can be useful to perform sensitivity analysis of the outputs with respect to the desired inputs, as well as to analyse the process response to certain inputs.

The What-If simulation can be used to experiment the effects over $\mathrm{H}_{2}$ efficiency of different operational strategies. It is complemented with a set of defined resource efficiency indicators (REIs) intended for on-line decision support. Proposed REls have been defined following certain rational principles: are based on a material-flow analysis MFA, computed per unit of product, and normalized to best case. Two REls have been selected as the most interesting.

The first REI1 is an intensity index ( $R E I_{\text {Resource Per Product }}$ ), independent of the make-up $\mathrm{H}_{2}$ quality and flow rate, only providing information about the network and plants state, but not accounting for efficiency.

$$
R E I 1_{H 2 \text { Net }}^{\text {Intensity }}=\frac{\sum_{\text {producer plants }} \text { pure } \mathrm{H}_{2} \text { produced }\left(\mathrm{Nm}^{3} / \mathrm{h}\right)}{\sum_{\text {consumer plants }} \text { hydrocarbon processed }\left(\mathrm{m}^{3} / \mathrm{h}\right)}
$$

$\mathrm{H}_{2}$ consumption in hydrotreatment plants is mainly influenced by: i) feedstock sulfur content; ii) desired naphtha/diesel grade, with low or high sulfur product specification according to its final use as either transport fuel or heating fuel; iii) type of hydrocarbon processed, and specially the light-cyclic-oil LCO content, due to double-bounds leading to higher $\mathrm{H}_{2}$ 
consumption. All of these factors should be included (with a more complex "equivalent hydrocarbon load"), or at least monitored together, in the aforementioned intensity index to make it more representative and also useful for comparison and validation purposes. The main difficulty is the great variability these factors show, and the lack of on-line measurements for feedstock sulfur content.

The most meaningful and representative of efficiency in $\mathrm{H}_{2}$ use is a relative REI3 compared to a best case ( $\left.\mathrm{REI}_{\text {norm }}\right)$, thus ensuring directional consistency, where Minimum Required Material or Reference is assigned as the minimum make-up or production to fulfil the $\mathrm{H}_{2}$ requirement constraints, obtained by solving an optimization problem with a material balance model of the $\mathrm{H}_{2}$ network. A standard KPI (REI1) only considering the production of $\mathrm{H}_{2}$ per hydrocarbon processed would fail to provide information about efficiency in operation, as constraints determine to a great extent both $\mathrm{H}_{2}$ requirements and the possibility of taking advantage of all the available $\mathrm{LPH} \mathrm{H}_{2}$.

$R E I 3_{H 2 \text { Net }}^{\text {REL efic }}=\frac{\sum_{\text {producer Min. Required Material non-pure } H_{2} \text { produced }(\text { Ref. })}}{\sum_{\text {producer plants }} \text { Actual Material non-pure } H_{2} \text { produced }}($ eq. 4$)$

Even though the use of performance indices (KPIs/REls) is extended at management level for medium-long time scales, its use for improving on-line process performance is not widespread, due to several reasons: i) the high degree of integration in process plants; ii) the difficulty to know the accurate value of the resources employed at each stage; iii) the lack of on-line measurements, especially regarding product quality and composition; iv) the difficulty to implement and maintain large model-based optimization applications when required to compute the best case to compare with as in this case; v) the demanding automation requirements to take full advantage of on-line overall optimal policies.

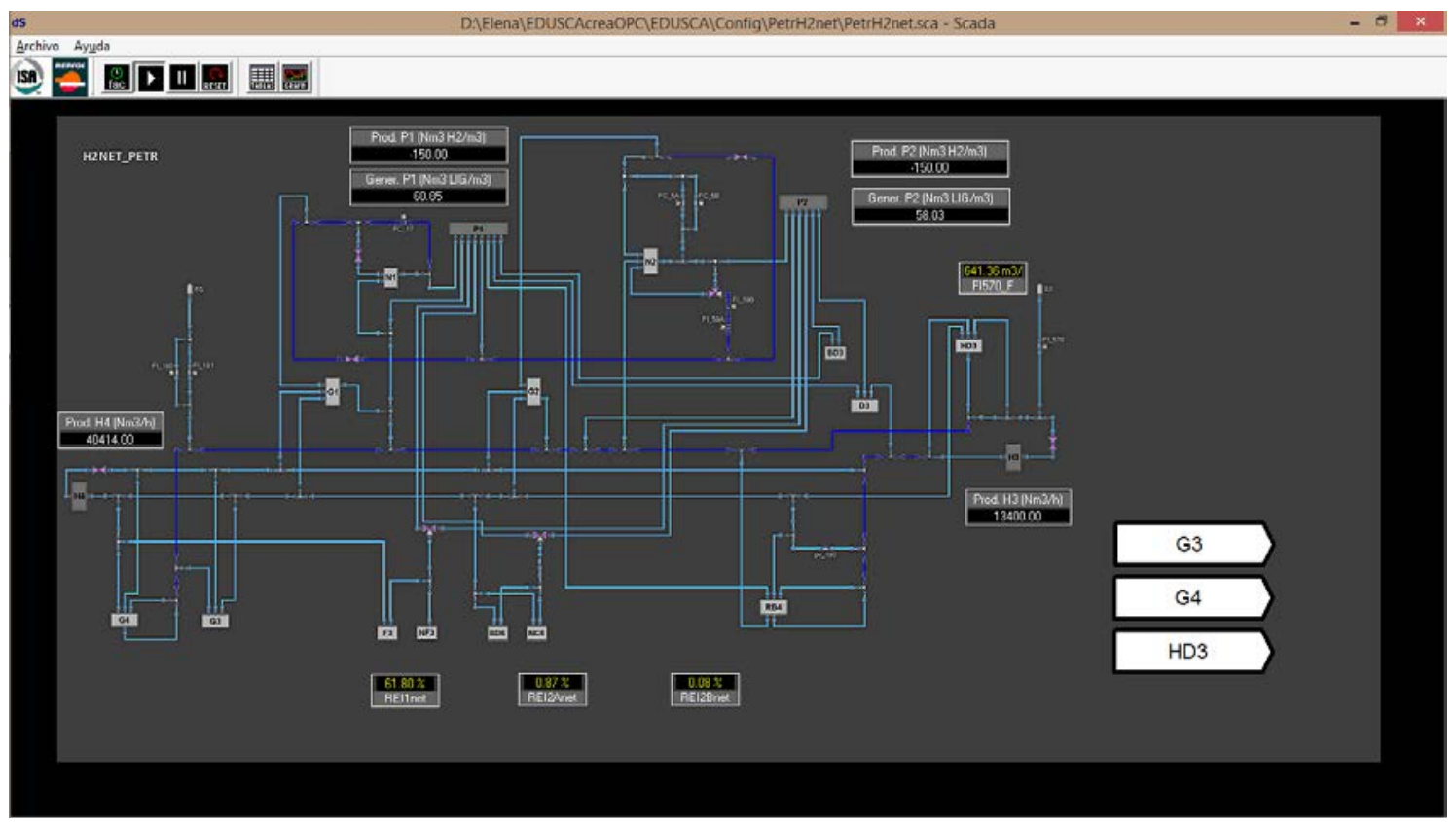



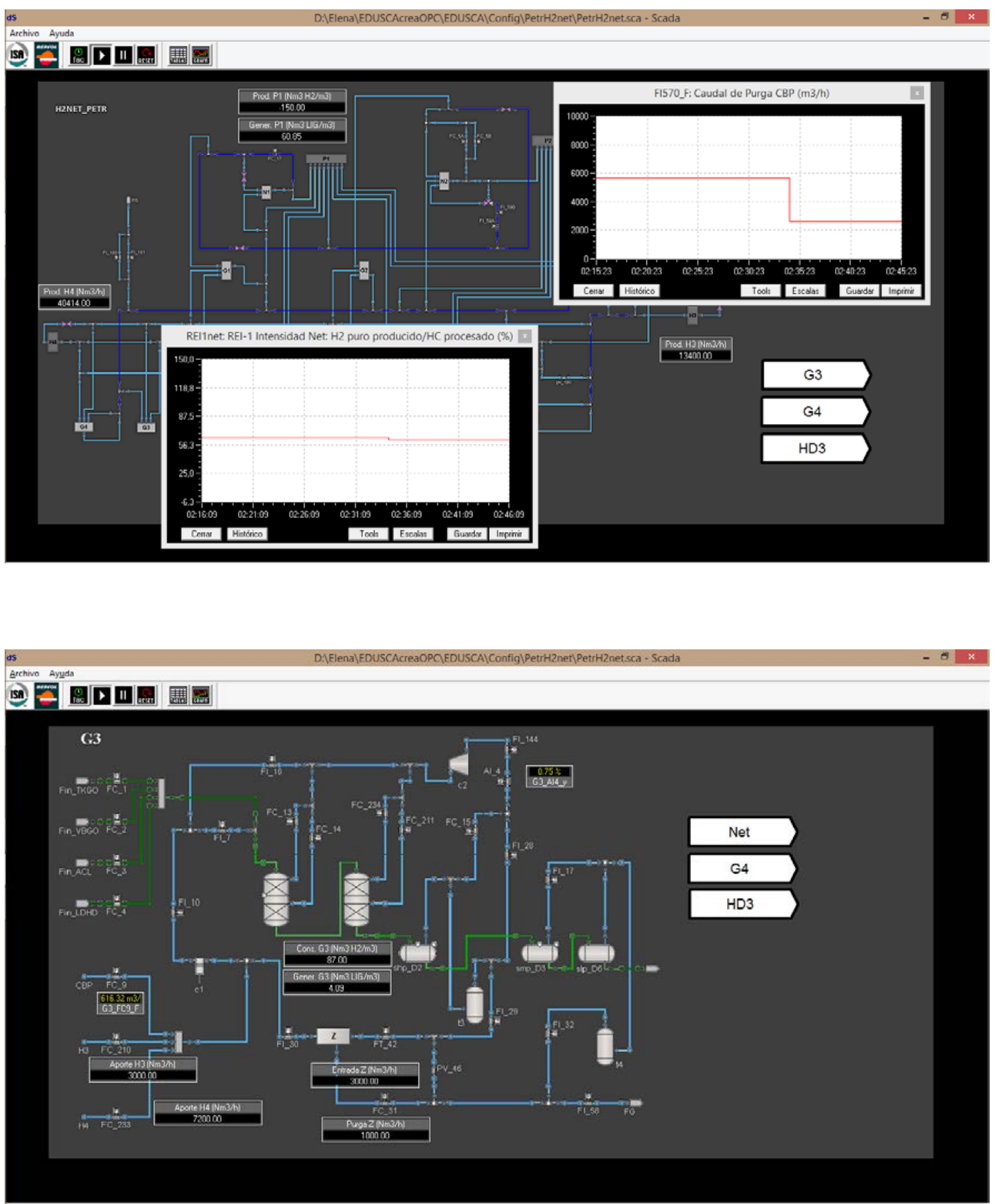

Figure 6.9. Screenshot of the EDUSCA ${ }^{\circledR}$ SCADA configuration for the $\mathrm{H}_{2}$ network problem, complemented with REls computation.

\section{7- CONCLUSIONS}

In summary, an analysis of solutions for the optimal $\mathrm{H}_{2}$ redistribution showed that: a) regarding high pressure (HP) purges, the solution is the logical one, that is, to purge Low Purity 
Header LPH excess, if any, at the network scope through the HP purge to FG at the lowest $\mathrm{H}_{2}$ purity until it gets saturated, following an increasing order of $\mathrm{H}_{2}$ purity to purge in consumer plants or headers; b) regarding trade-offs arising in redistribution from producer plants (high purity, expensive) and LPH (low purity, cheap) to consumer plants, there is margin for profit although small. Nevertheless the RTO approach can prove advantageous due to frequent changes in scenarios, aiding the operators to save time in the identification and implementation of the optimal policy.

With respect to the approach and application, the following general conclusions can be stated:

- Well-known techniques have been used to solve the optimal $\mathrm{H}_{2}$ redistribution problem.

- The model is a first-principles one, therefore reliable for prediction purposes. Modelling assumptions with the highest uncertainty are those corresponding to solubility parameters; however, losses downstream the HP separator liquid outlet account for about $10 \%$ of the total $\mathrm{H}_{2}$ production, therefore even for important prediction errors or model mismatch, results regarding redistribution will be accurate enough. Moreover, consumption in reactors account for about $85 \%$ of the total $\mathrm{H}_{2}$ production and can be estimated with certain accuracy due to redundant flowmeters and on-line analysers in the HP system, thus enabling certain reliability in the optimal redistribution results.

- Further work should be done regarding computational efforts. The execution time with the implementation used is too high; either EcosimPro ${ }^{\circledR}$ is complemented with automatic differentiation or the EcosimPro ${ }^{\circledR}$ model is converted to another environment providing automatic differentiation as for example $\operatorname{CppAD}^{\circledR}$, with exact first and second derivatives and high execution times.

- An alternative approach to solve the optimal redistribution problem by means of a non-linear MPC based on the first-principles model could be interesting, in particular if all decision variables (flow rates) could be automatically manipulated. It should act on an upper layer in the control hierarchy, with a slower time-scale than the currently implemented advanced regulatory control MPC applications.

Other approaches could also be studied, as for example the self-optimizing technique, taking advantage of the fact that the biggest potential for profit margin corresponds to the HP purges to FG, and could be easily programmed with a logic structure.

- Furthermore, the analysis could be also valuable to reveal economic-technical trade-offs, where non-linear behaviours arise. Because of an operation at an increased HP system $\mathrm{H}_{2}$ purity $\mathrm{Y}_{\text {HPsep }}{ }^{\mathrm{H} 2}$ in consumer plants, additional benefits can be achieved together with a higher-value processed hydrocarbon. The underlying relations regarding $\mathrm{H}_{2}$ requirements are non-linear, especially considering that it is the $y_{\text {HPsep }}{ }^{\text {H2 }}$ constraint that determines whether there is a possibility to reuse 
available low-purity $\mathrm{H}_{2}$, or on the contrary there is a need to get rid of it being useless to fulfil constraints. Although the main drawback is the difficulty to quantify the added value in hydrocarbon due to a higher $y_{\text {нрsеp }}{ }^{\mathrm{H}}$ in process operation, a study exploring certain alternatives in a Pareto front could be rational. 


\section{CHAPTER 7}

\section{CONCLUSIONS}

\section{1- CONCLUSIONS}

Efficiency in the use of raw materials and energy, resources in general, is a common concern in process industry. Nowadays, increasing interest exists in optimization applications for plantwide operation, taking advantage of the current high automation levels in the lower layers of the control hierarchy, and with the aim of increasing the demanding profit margins.

Plantwide simplified models for the $\mathrm{H}_{2}$ distribution network of an oil refinery have been developed and validated, intended for steering process operation towards optimality in a decision-support system for the on-line optimal management. The $\mathrm{H}_{2}$ network is a complex system, with strong interactions and where operating constraints must be taken into account to guarantee feasibility in the real operation.

The feasibility of the approach has been shown. The most significant results of this study are summarized below, according to different topics.

\section{General}

- A large scale industrial problem has been addressed, using standard commercial tools for modelling, simulation and optimization. Although the degree of complexity of the problems tackled is not high, and well-known techniques of chemical process modelling and optimization have been applied, the number of variables involved, the uncertainty, and the problems dimension are a challenge.

- A library with optimization capabilities was developed in the EcosimPro ${ }^{\circledR}$ environment. The network model can be easily built graphically and parameters configured also graphically. Development issues regarding the implementation have been dealt with. Routines were developed for a quasi-automatic generation of the code needed to implement the optimization problems from the graphical model.

- In a cooperation project with the Repsol ${ }^{\circledR}$ Technology Center CTR in Móstoles (Madrid), the model and optimization capabilities have also been used for 
revamping purposes, where different previously screened alternatives regarding new connections, recycling of important medium-pressure purges and membranes use were analysed, and the savings quantified by optimization techniques for a proposed fixed scenario.

- The problem of the $\mathrm{H}_{2}$ network optimal management -comprising the model, simulation and optimizations implementations- is being used as case study in the European project MORE "Real-time Monitoring and Optimization of Resource Efficiency in Integrated Processing Plants", from November 2013 until November 2016, intended for a real-time monitoring of the efficiency in the material and energy resources use, and leaded by Prof. Sebastian Engell.

\section{Modelling}

- A plantwide model for the $\mathrm{H}_{2}$ network of a petrol refinery has been developed, calibrated and validated against real industrial data. The model is based on conservation laws and first principles, balancing simplicity with magnitude consistency and physical meaning. The degree of model complexity is appropriate taking into account available on-line measurements (few regarding composition) and high process variability; it can be on-line calibrated and updated; moreover, it is able to provide good performance according to the aim pursued.

The first-principles model provides the advantage of consistent prediction capabilities, which is an interesting feature due to the strong non-linear behaviours and interactions at the network scope.

- Most of the proposed parameters are physically meaningful, in particular those for reactors and separators, therefore aiding in process understanding and useful for comparison purposes among similar units.

- The gas stream model with two allowed directions for flow provides certain flexibility in the optimization regarding topological decisions, whereas entailing a simple approach as compared to a hybrid modelling with binary decision variables.

The network operation regarding connections and topological decisions is maintained, i.e., boolean decision variables are not considered in the optimization thus not being a hybrid problem. Nevertheless, it is not a restrictive assumption as aforementioned.

- Identifiability issues have been addressed with narrow limits in parameters when appropriate according to process knowledge and operating conditions, thus reducing the effects of overparameterization. The reason is the high influence of gas molecular weight $w$ over the compensation of the raw measurement flow rate, used as reference for model fit. 
Regarding the HP system, the model is formally overparameterized, although in practice consistent results are obtained for most of the streams due to: i) significant redundancy (\%) in flow measurements; ii) the existence of on-line analysers in both big and medium size plants; iii) the bounded variability for the generated light ends molecular weights $\Delta R_{w L I G}$, with a reduced range with respect to the expected in operation; iv) narrow bounds for certain parameters according to process knowledge and operating conditions: ratio light ends generation to $\mathrm{H}_{2}$

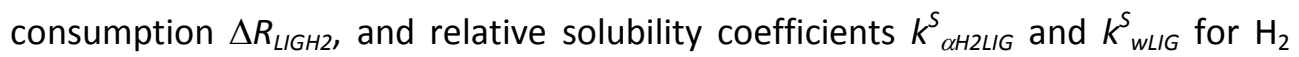
and light ends distribution between phases.

Regarding the MP/LP systems, the model is overparameterized due to the lack of on-line analysers for $\mathrm{H}_{2}$ purity and stream molecular weight, together with a smaller degree of flowmeters redundancy than in the HP system; moreover, process variability in composition is significantly higher as compared with the HP system, according to historical laboratory data. Nevertheless, the gas outlet flow rate has a strong influence over the solubility equilibria achieved, being measured in spite of compensation uncertainty and potential drift errors, thus providing a valuable reference.

- Validation in plant has not been carried out yet.

\section{Data Reconciliation}

The well-known technique of process state estimation by means of the minimization of an objective function composed of model-measurement deviations, WLS weighted least squares, benefits from dealing with all the available on-line information. The problem addressed is challenging due to uncertainty: error prone flowmeters, lack of on-line measurements for $\mathrm{H}_{2}$ purity and gas composition, mixtures complexity with strong influence over flow measurements compensation, and significant process variability. A rational approach has been applied to the $\mathrm{H}_{2}$ network according to parameter sensitivities and problems encountered.

- The underlying model based on conservation laws has enabled to detect and disregard instruments affected by gross errors in an off-line analysis by simple inspection, because gross errors are persistent in time, and constant in sign and approximately magnitude (systematic).

- Validation was accomplished by comparison with raw measurement trends for all the most important variables along different periods, with satisfactory results.

- Model parameters and bounds have been finely tuned to achieve good performance in the global network fitting, guaranteeing consistency and robustness of results at the expense of a more reduced flexibility than expected according to process variability in certain model parameters, although not compromising the accuracy of the results. Further improvements will be difficult to accomplish due to: i) inescapable local optima; ii) a certain degree of 
overparameterization and poor identifiability; iii) uncertainty in on-line measurements.

Although unbiased estimates cannot be guaranteed with the WLS technique in the presence of non-random errors, potential drift and bias errors in flowmeters $\mathrm{FI}$ and analysers $\mathrm{Al}$ do not affect the reconciled results badly. Narrow ranges for certain $\mathrm{Fl}$ and $\mathrm{Al}$ have proved determining, again due to multiple local optima and interactions. Different treatments for different variables according to their type $(\mathrm{Al} / \mathrm{FI})$ and sensitivity have been applied; accepted tolerance in measurements has proved important in the data reconciliation step, in particular allowed range for Als measurement error, because due to their high sensitivity this tolerance determines to a great extent the achieved fit in the whole set of Fls.

\section{Optimal Redistribution}

The feasibility of the RTO approach for the on-line optimal operation of the $\mathrm{H}_{2}$ network was proved, and reasonable results were achieved ready to be applied in the real operation.

- The approach is to compute certain model parameters and variables in the Data Reconciliation step and fixed them as inputs to solve the Optimal Redistribution problem, where assumptions made are reasonable enough.

An analysis of solutions shows that: a) regarding HP purges, the solution is the logical one, that is, to purge LPH (Low Purity Header) excess (if any) at the network scope through the HP purge at lower $\mathrm{H}_{2}$ purity until it gets saturated, following an increasing order of $\mathrm{H}_{2}$ purity to purge in consumer plants; b) regarding trade-offs arising in optimal redistribution of $\mathrm{H}_{2}$ from producer plants (high purity, expensive) and LPH (low purity, cheap) to consumer plants, there is margin for profit although small. Nevertheless, the tool can prove advantageous due to frequent changes in scenarios, aiding to save time in the identification of suboptimal operation and implementation of optimal policies.

- The model is a first-principles one, therefore reliable for prediction purposes. Modelling assumptions with the highest uncertainty are those corresponding to solubility parameters; however, losses downstream the HP separator liquid outlet account for about $10 \%$ of the total $\mathrm{H}_{2}$ production, therefore even for important prediction errors or model mismatch, results regarding redistribution will be accurate enough. Moreover, consumption in reactors account for about $85 \%$ of the total $\mathrm{H}_{2}$ production and can be estimated with certain accuracy due to redundant flowmeters and on-line analysers in the HP system, thus enabling certain reliability in the optimal redistribution results.

- As compared to other commercially available tools which have been recently developed based on Hysys ${ }^{\circledR}$ process simulator and its optimization capabilities, most probably the same rigorous and systematic approach for the optimal redistribution problem is shared regarding optimization techniques, important 
assumptions and process constraints; however to the best of our knowledge, data reconciliation is not performed taking advantage of redundancy with optimization techniques, but just considering the sufficient number of inputs. A flexible and easily updated model calibration is worthwhile, which can be enhanced with an intended simple model as the one developed.

\section{2- FUTURE WORK}

Certain open issues have arisen which would be interesting to further explore. Topics for future research are organized below according to modelling, control and optimization.

\section{Modelling}

- With the purpose of allowing increased process variability in certain model parameters while at the same time not compromising robustness and consistency, correlations on a coarse level as a function of measurable variables with significance could be studied. In particular, correlations for: i) molecular weight of light ends generated in catalytic-reforming producer plants as a function of both reactor temperature and feed quality; ii) relative solubility coefficients in the HP separator for important consumer plants as a function of both operation temperature and product composition, to improve the accuracy in the equilibrium estimation.

\section{Control}

- An extension to the whole network of the self-optimizing technique (Skogestad, 2000) for process optimization could be thought of, taking into account that the highest potential for profit margin is due to logical decisions regarding the HP purges to FG, whereas margin in a preferential make-up from either one header or another to a certain consumer plant is significantly lower due to similar prices and purities of the $\mathrm{H}_{2}$ produced in the steam-reforming furnace producer plants.

In this regard of the HP purges, optimal decisions regarding the manipulated variables are logical, therefore could be easily managed according to rules where states and transit conditions are programmed with a logic structure. This solution should be applied in a layer above the basic regulatory control and the advanced MPC applications, acting on a slower time-scale.

- An alternative approach to solve the optimal redistribution problem by means of a non-linear MPC based on the first-principles model could be interesting to explore, in particular if all decision variables (flow rates) could be automatically manipulated. It should act on an upper layer in the control hierarchy, with a 
slower time-scale than the currently implemented advanced regulatory control MPC applications. The feasibility and potential advantages of a MPC application to control the whole network could be previously analysed and checked in simulation.

\section{Optimization}

- Strategies for optimization under uncertainty could be studied in order to increase robustness against measurement errors.

- Further work should be done regarding computational efforts, since the execution time with the implementation used is too high. Other environments or solvers are being tested for use with EcosimPro ${ }^{\circledR}$ models, in particular the $\operatorname{CppAD}^{\circledR}$ optimization environment providing exact first and second order derivatives by means of automatic differentiation, with a simultaneous approach to solve the modelling equations together with the optimization problem. The cooperation of the EcosimPro ${ }^{\circledR}$ team for an automatic syntax conversion of the modelling code is already planned.

- Compression energy cost in the $\mathrm{H}_{2}$ network is roughly about $10-20 \%$ that of $\mathrm{H}_{2}$ material cost, and further research could be worthy regarding the inclusion of both energy and material resources in the cost objective function to minimize for optimal operation.

In a decoupled approach, efficiency in the use of energy in different compressors can be useful for establishing rules to aid in operations optimization, which implies mainly to assign low purity $\mathrm{H}_{2}$ (with higher flow rate requirements) among multiple consumer plants to compressors with the highest efficiency, provided it is not detrimental to the material efficiency. This further refinement would also prompt that consumer plants are fed with $\mathrm{H}_{2}$ from the nearest producer plant, so as to minimize transport cost. Nevertheless, both material and energy efficiency should be considered jointly to operate the process according to a single performance criterion, in particular if considered that decisions regarding material and energy efficiency cannot be decoupled because of a trade-off.

- The economic-technical trade-offs, where non-linear behaviours arise, deserve to be further explored. Because of an operation at an increased HP system $\mathrm{H}_{2}$ purity $y_{H P s e p}{ }^{H 2}$ in consumer plants, additional benefits can be achieved together with a higher-value processed hydrocarbon. The underlying relations regarding $\mathrm{H}_{2}$ requirements are non-linear, especially considering that it is the $y_{\text {нрsеp }}{ }^{H 2}$ constraint that determines whether there is a possibility to reuse available low-purity $\mathrm{H}_{2}$, or on the contrary there is a need to get rid of it being useless to fulfil constraints. Although the main drawback is the difficulty to quantify the added value in hydrocarbon due to a higher $y_{\text {HPsep }}{ }^{H 2}$ in the reactor operation, a study exploring certain alternatives in a Pareto front could be rational. 


\section{REFERENCES}

ACEBES, L.F., MERINO, A., ALVES, R. (2009). Online energy diagnosis of sugar plants. Revista Iberoamericana de Automática e Informática Industrial, 6 (3), 68-.

ACEDO SANCHEZ, J. (2006). Instrumentación y control avanzado de procesos. Ed. Díaz de Santos.

ADETOLA, V., GUAY, M. (2010). Integration of real-time optimization and model predictive control. J. Process Control, 20 (2), 125-133.

AGARWAL, A., BIEGLER, L.T., ZITNEY, S.E. (2009). Simulation and optimization of pressure swing adsorption systems using reduced-order modeling. Ind. Eng. Chem. Res., 48 (5), 23272343.

AGARWAL, V., SHENOY, U.V. (2006). Unified conceptual approach to targeting and design of water and hydrogen networks. AIChE J., 52 (3), 1071-1082.

AHMAD, M.I., ZHANG, N., JOBSON, M. (2010). Modelling and optimisation for design of hydrogen networks for multi-period operation. J. Clean. Prod., 18 (9), 889-899.

ALAMO, T., FERRAMOSCA, A., GONZALEZ, A.H., LIMON, D., ODLOAK, D. (2014). A gradientbased strategy for the one-layer RTO plus MPC controller. J. Process Control, 24 (4), 435-447.

ALSTAD, V., SKOGESTAD, S. (2007). Null space method for selecting optimal measurement combinations as controlled variables. Ind. Eng. Chem. Res., 46 (3), 846-853.

ALSTAD, V., SKOGESTAD, S., HORI, E.S. (2009). Optimal measurement combinations as controlled variables. J. Process Control, 19, 138-148.

ALVES, J. (1999). Analysis and design of refinery hydrogen systems. Ph.D. Thesis, UMIST.

ALVES, J.J., TOWLER, G.P. (2002). Analysis of refinery hydrogen distribution systems, Ind. Eng. Chem. Res., 41 (23), 5759-5769.

AMRIT, R., RAWLINGS, J.B., Biegler, L.T. (2013). Optimizing process economics online using model predictive control. Comput. Chem. Eng., 58, 334-343. 
ARAUJO, A.C.B., GOVATSMARK, M., SKOGESTAD, S. (2007). Application of plantwide control to the HDA process. I Steady-state and self-optimizing control. Control Eng. Pract., 15, $1222-1237$.

ASKE, E.M.B., SKOGESTAD, S. (2009). Consistent inventory control. Ind. Eng. Chem. Res., 48(44), 10892-10902.

BAGAJEWICZ, M. (2005a). On the definition of software accuracy in redundant measurement systems. AlChE J., 51 (4), 1201-1206.

BARTELS, J.R., PATE, M.B., OLSON, N.K. (2010). An economic survey of hydrogen production from conventional and alternative energy sources. Int. J. Hydrogen Energy, 35, 83718384.

BELLOS, G.D., KALLINIKOS, L.E., GOUNARIS, C.E., PAPAYANNAKOS, N.G. (2005). Modelling of the performance of industrial HDS reactors using a hybrid neural network approach. Chem. Eng. Proc., 44, 505-515.

BHASKAR, M., VALAVARASU, G., SAIRAM, B., BALARAMAN, K.S., BALU, K. (2004). Three-phase reactor model to simulate the performance of pilot-plant and industrial trickle-bed reactors sustaining hydrotreating reactions. Ind. Eng. Chem. Res., 43 (21), 6654-6669.

BIEGLER, L.T., YANG, X., FISCHER, G.A.G. (2015). Advances in sensitivity-based nonlinear model predictive control and dynamic real-time optimization. J. Process Control, 30, 104-116.

BIRJANDI, M.R.S., SHAHRAKI, F., BIRJANDI, M.S., NOBANDEGANI, M.S. (2014). Application of global optimization strategies to refinery hydrogen network. Int. J. Hydrogen Energy, 39, 14503-14511.

BOHLIN, T. (2006). Practical grey box process identification. Springer.

BUSSANI, G., CHIARI, M., GROTTOLI, M.G., PIERUCCI, S., FARAVELLI, T., RICCI, G., GIOVENTLI, G. (1995). Application of data-reconciliation and optimisation procedure to hydrogen plant. Comput. Chem. Eng., 19, 299-304.

CAI, H.-Y., SHAW, J.M., CHUNG, K.H. (2001). Hydrogen solubility measurements in heavy oil and bitumen cuts. Fuel, 80, 1055-1063.

CHACHUAT, B., SRINIVASAN, B., Bonvin, D. (2009). Adaptation strategies for real-time optimization. Comput. Chem. Eng., 33, 1557-1567.

CHEN, J., ROMAGNOLI, J.A. (1998). A strategy for simultaneous dynamic data reconciliation and outlier detection. Comput. Chem. Eng., 22 (4/5), 559-562.

CHEN, L., BERNARD, O., BASTIN, G., ANGELOV, P. (2000). Hybrid modelling of biotechnological processes using neural networks. Control Eng. Pract., 8, 821-827.

CHENG, Z., FANG, X., ZENG, R., HAN, B., HUANG, L., YUAN, W. (2004). Deep removal of sulfur and aromatics from diesel through two-stage concurrently and countercurrently operated fixed-bed reactors. Chem. Eng. Sci., 59, 5465-5472. 
CHIARI, M., BUSSANI, G., GROTTOLI, M.G., PIERUCCI, S. (1997). Online data reconciliation and optimisation: refinery applications. Comput. Chem. Eng., 21, 1185-1190.

CHOWDHURY, R., PEDERNERA, E., REIMERT, R. (2002). Trickle-bed reactor model for desulfurization and dearomatization of diesel. AIChE J., 48 (1), 126-135.

COULSON, J.M. (1996). Chemical Engineering. Vol. 6: Chemical Engineering Design. Pergamon.

CREUS, A. (1997). Instrumentación Industrial. Marcombo, 6a ed.

CROWE, C.M. (1996). Data reconciliation - progress and challenges. J. Process Control, 6, 8998.

DANIEL MEASUREMENT AND CONTROL, Inc. Application of the Orifice Meter for Accurate Gas Flow Measurement. Emerson Process Management.

DARBY, M.L., NIKOLAOU, M., JONES, J., NICHOLSON, D. (2011). RTO: An overview and assessment of current practice. J. Process Control, 21, 874-884.

DOWNS, J.J., SKOGESTAD, S. (2011). An industrial and academic perspective on plantwide control. Annual Reviews in Control, 35, 99-110.

DUVALL, P.M., RIGGS, J.B. (2000). On-line optimization of the Tennessee Eastman challenge problem. J. Process Control, 10, 19-33.

EDGAR, T.F., HIMMELBLAU, D.M., LASDON, L.S. (2001). Optimization of chemical processes. McGraw-Hill, $2^{\text {nd }}$ ed.

EL-HALWAGI, M.M., GABRIEL, F., HARELL, D. (2003). Rigorous graphical targeting for resource conservation via material recycle/reuse networks. Ind. Eng. Chem. Res., 42 (19), 43194328.

ELLIS, M., CHRISTOFIDES, P.D. (2014). Integrating dynamic economic optimization and model predictive control for optimal operation of nonlinear process systems. Control Eng. Pract., 22, 242-251.

EL-TWATY, A.I., PRAUSNITZ, J.M. (1980). Correlation of k-factors for mixtures and heavy hydrocarbons. Chem. Eng. Sci., 35, 1765-1768.

ENGELL, S. (2007). Feedback control for optimal process operation. J. Process Control, 17, 203219.

ESCHE, E., MÜLLER, D., KRAUS, R., WOZNY, G. (2014). Systematic approaches for model derivation for optimization purposes. Chem. Eng. Sci., 115, 215-224.

FABER, R., ARELLANO-GARCIA, H., LIB, P., WOZNY, G. (2007). An optimization framework for parameter estimation of large-scale systems. Chem. Eng. Proc., 46, 1085-1095.

FABER, R., LI, P., WOZNY, G. (2003). Sequential parameter estimation for large-scale systems with multiple data sets. Part I. Computational framework. Ind. Eng. Chem. Res., 42 (23), 5850-5860. 
FABER, R., LI, B., LI, P., WOZNY, G. (2006). Data reconciliation for real-time optimization of an industrial coke-oven-gas purification process. Simulation Modelling Practice and Theory, 14 (8), 1121-1134.

FONSECA, A., SA, V., BENTO, H., TAVARES, M.L.C, PINTO, G., GOMES, L.A.C.N. (2008). Hydrogen distribution network optimization: a refinery case study. J. Clean. Prod., 16 (16), 17551763.

FOO, D.C.Y., KAZANTZI, V., EL-HALWAGI, M.M., MANAN, Z.A. (2006). Surplus diagram and cascade analysis technique for targeting property-based material reuse network. Chem. Eng. Sci., 61 (8), 2626-2642.

FORBES, J.F., MARLIN, T.E. (1996). Design cost: a systematic approach to technology selection for model-based real-time optimization systems. Comput. Chem. Eng., 20, 717-734.

FORBES, J.F., MARLIN, T.E., MACGREGOR, J.F. (1994). Model adequacy requirements for optimizing plant operations. Comput. Chem. Eng., 18, 497-510.

FORSBERG, C.W. (2005). The Hydrogen Economy is Coming The Question is Where? Chem. Eng. Progress, 101 (12), 20-.

FROMENT, G.F., DEPAUW, G.A., VANRYSSELBERGUE, V. (1994). Kinetic modeling and reactor simulation in hydrodesulfurization of oil fractions. Ind. Eng. Chem. Res., 33 (12), 29752988.

GEORGIEVA, P., MEIRELES, M.J., FEYO DE AZEVEDO, S. (2003). Knowledge-based hybrid modelling of a batch crystallization when accounting for nucleation, growth and aglomeration phenomena. Chem. Eng. Sci., 58, 3699-3713.

GHOSH, A., CHAPMAN, W.G., FRENCH, R.N. (2003). Gas solubility in hydrocarbons-a SAFTbased approach. Fluid Phase Equilibria, 209, 229-243.

GMEHLING, J., ONKEN, U., ARLT, W. (1981). Vapor-Liquid equilibrium data collection, Chemistry data series. Vol. I, Part 1a, Frankfurt, DECHEMA.

GOMEZ SAYALERO, E., SARABIA, D., CRISTEA, S., GUTIERREZ, G., MENDEZ, C.A., SOLA, J.M., UNZUETA, E., GONZALEZ, R., DE PRADA, C. (2010). Simplified modelling and validation of an industrial diesel hydrodesulfurization plant. DYCOPS 9th Dynamics \& Control of Systems, Louvain, Belgium.

GOMEZ SAYAleRO, E., DE PRADA, C., SARABIA, D., MENDEZ, C.A., CRISTEA, S., SOlA, J.M., UNZUETA, E., GONZALEZ, R. (2008). Grey-box modelling of an industrial hydrodesulphurization process. $18^{\text {th }}$ European Symposium on Computer Aided Process Engineering - ESCAPE 18. Lyon, Editors Bertrand Braunschweig and Xavier Joulia. Elsevier B.V./Ltd. ISBN 978-0-444-53228-2.

GOMEZ SAYALERO, E., SKOGESTAD, S., DE PRADA, C., SOLA, J.M., GONZALEZ, R. (2012). Selfoptimizing control for hydrogen optimization in a diesel hydrodesulfurization plant. 
PSE 11th International Symposium on Process Systems Engineering. Singapore, Singapore.

GOMEZ SAYALERO, E., GUTIERREZ, G., SARABIA, D., DE PRADA, C., MARMOL, S., SOLA, J.M., PASCUAL, C., GONZALEZ, R. (2014). Optimal operation of a petrol refinery hydrogen network. IFORS 20th Conference of the International Federation of Operational Research Societies. Barcelona, Spain.

GOMEZ SAYALERO, E., GUTIERREZ, G., SARABIA, D., MARMOL, S., SOLA, J.M., PASCUAL, C., GONZALEZ, R., DE PRADA, C. (2015). Resource efficiency indicators applied to refinery hydrogen networks. ECCE 10th European Congress of Chemical Engineering. Nice, France.

GRACIANO, J.E.A., JÄSCHKE, J., LE ROUX, G.A.C., BIEGLER, L.T. (2015). Integrating selfoptimizing control and real-time optimization using zone control MPC. J. Process Control, 34, 35-48.

GUtierReZ, G., SARABIA, D., DE PRADA, C., GOMEZ, E., CRISTEA, S. (2010). Optimal management of a hydrogen network in petrol refinery. $20^{\text {th }}$ European Symposium on Computer Aided Process Engineering, Ischia, Nápoles, Italia.

HALLALE, N., LIU, F. (2001). Refinery hydrogen management for clean fuels production. Advances in Environmental Research, 6, 81-98.

HALVORSEN, I.J., SKOGESTAD, S., MORUD, J.C., ALSTAD, V. (2003). Optimal selection of controlled variables. Ind. Eng. Chem. Res., 42 (14), 3273-3284.

HIMMELBLAU, D.M., BISCHOFF, K.B. (1992). Análisis y simulación de procesos. Ed. Reverté.

IDRIS, E.A.N., ENGELL, S. (2012). Economics-based NMPC strategies for the operation and control of a continuous catalytic distillation process. J. Process Control, 22 (10), 18321843.

ISA-ESPAÑA. (2008). Medida de caudal. Curso de formación.

ISO 5167-1 (1991). Measurement of fluid flow by means of pressure differential devices - Part 1. Orifice plates nozzles and Venturi tubes inserted in circular cross section conduits running full. Geneve.

JIAO, Y., SU, H., HOU, W. (2012). Improved optimization methods for refinery hydrogen network and their applications. Control Eng. Pract., 20, 1075-1093.

JIAO, Y., SU, H., HOU, W., LIAO, Z. (2012). Optimization of refinery hydrogen network based on chance constrained programming. Chem. Eng. Res. Des., 90, 1553-1567.

JIAO, Y., SU, H., LIAO, Z., HOU, W. (2011). Modeling and Multi-objective Optimization of Refinery Hydrogen Network. Chinese Journal of Chemical Engineering, 19 (6), 990-998.

JOCKENHOVEL, T., BIEGLER, L.T., WACHTER, A. (2003). Dynamic optimization of the Tennessee Eastman process using the OptControlCentre. Comput. Chem. Eng., 27 (11), 1513-1531. 
JUN, Z., ZHI-ZHONG, M., RUN-DA, J. (2015). Real-time optimization based on SCFO for gold cyanidation leaching process. Chem. Eng. Sci., 134, 467-476.

KELLY, J.D. (2004). Techniques for solving industrial nonlinear data reconciliation problems. Comput. Chem. Eng., 28 (12), 2837-2843.

KELLY, J.D. (2004). Formulating large-scale quantity-quality bilinear data reconciliation problems. Comput. Chem. Eng., 28 (3), 357-362.

KHAJEHPOUR, M., FARHADI, F., PISHVAIE, M.R. (2009). Reduced superstructure solution of MINLP problem in refinery hydrogen management. Int. J. Hydrogen Energy, 34 (22), 9233-9238.

KIM, I.-W., LIEBMANN, M.J., EDGAR, T.F. (1990). Robust error-in-variables estimation using nonlinear programming techniques, AIChE J., 36 (7), 985.

KORSTEN, H., HOFFMANN, U. (1996). Three-phase reactor model for hydrotreating in pilot trickle-bed reactors. AIChE J., 42, 1350-1360.

KUMAR, A., GAUTAMI, G., KHANAM, S. (2010). Hydrogen distribution in the refinery using mathematical modeling. Energy, 35, 3763-3772.

LAURET, P., BOYER, H., GATINA, J.C. (2000). Hybrid modelling of a sugar boiling process. Control Eng. Pract., 8, 299-310.

LEE, S., GROSSMANN, I.E. (2003). Global optimization of nonlinear generalized disjunctive programming with bilinear equality constraints: applications to process networks. Comput. Chem. Eng., 27 (11), 1557-1575.

LEE, Y.K., PARK, H.K., JEONG, C.H., LEE, J.R., MINTER, B.J., GOODHART, S.G. (2008). Control and optimization of a large scale refinery hydrogen network. Proceedings of the 17th World Congress The International Federation of Automatic Control. Seoul, Korea, July 6-11.

LEVENSPIEL, O. (1998). Ingeniería de las reacciones químicas, 2aed. Ed. Reverté.

LIAO, Z.W., RONG, G., WANG, J.D., YANG, Y.R. (2011). Rigorous algorithmic targeting methods for hydrogen networks part I: systems with no hydrogen purification. Chem. Eng. Sci., 66 (5), 813-820.

LIAO, Z.W., RONG, G., WANG, J.D., YANG, Y.R. (2011). Rigorous algorithmic targeting methods for hydrogen networks part II: systems with one hydrogen purification. Chem. Eng. Sci., 66 (5), 821-833.

LIAO, Z., WANG, J., YANG, Y., RONG, G. (2010). Integrating purifiers in refinery hydrogen networks: a retrofit case study. J. Clean. Prod., 18, 233-241.

LID, T., SKOGESTAD, S. (2008). Data reconciliation and optimal operation of a catalytic naphtha reformer. J. Process Control, 18, 320-331. 
LIEBMAN, M.J., EDGAR, T.F., LASDON, L.S. (1992). Efficient data reconciliation and estimation for dynamic processes using nonlinear programming techniques. Comput. Chem. Eng., 16 -(10/11), 963-986.

LINNHOFF, B., TOWNSEND, D.W., BOLAND, D. et al. (1982). User guide on process integration for the efficient use of energy. IChemE, Rugby, UK.

LIU, F., ZHANG, N. (2004). Strategy of purifier selection and integration in hydrogen networks. Chem. Eng. Res. Des., 82 (A10), 1315-1330.

LUNG, L., GLAD, T. (1994). Modeling of dynamic systems. Prentice-Hall.

LUUNG, L. (1987). System identification: theory for the user. Prentice-Hall.

LUCKWAL, K., MANDAL, KUMAR, K. (2009). Improve hydrogen management of your refinery. Hydrocarbon Processing, 55-61.

LUDWIG, E.E. (1999, 1993, 1977). Applied process design for chemical and petrochemical plants. Vol. 1, Vol. 2, Vol. 3. Houston, Gulf.

LUYBEN, W.L. (1990). Process modeling, simulation, and control for chemical engineers. McGraw-Hill.

MAHALEC, V., SANCHEZ, Y. (2012). Inferential monitoring and optimization of crude separation units via hybrid models. Comput. Chem. Eng., 45, 15-26.

MANDELA, R.K., RENGASWAMY, R., NARASIMHAN, S., SRIDHAR, L.N. (2010). Recursive state estimation techniques for non linear differential algebraic systems. Chem. Eng. Sci., 65, 4548-4556.

MANDUR, J.S., BUDMAN, H.M. (2015). Simultaneous model identification and optimization in presence of model-plant mismatch. Chem. Eng. Sci., 129, 106-115.

MANENTI, F., GROTTOLI, M.G., PIERUCCI, S. (2011). Online data reconciliation with poor redundancy systems. Ind. Eng. Chem. Res., 50 (24), 14105-14114.

MARCHETTI, A., CHACHUAT, B., BONVIN, D. (2009). Modifier-adaptation methodology for realtime optimization. Ind. Eng. Chem. Res., 48 (13), 6022-6033.

MARCHETTIA, A.G., FERRAMOSCA, A., GONZALEZ, A.H. (2014). Steady-state target optimization designs for integrating real-time optimization and model predictive control. J. Process Control, 24, 129-145.

MASTROGIACOMO, M., BILODEAU, D., TREIBER, S., WALKER, J. (1997). Advanced control of hydrogen network reduces energy consumption. Oil \& Gas J., 95 (35), $72-$.

MEDEROS, F.S., RODRIGUEZ, M.A., ANCHEYTA, J., ARCE, E. (2006). Dynamic modeling and simulation of catalytic hydrotreating reactions. Energy \& Fuels, 20, 936-945. 
MEIJER, S.C.F., VAN DER SPOEL, H., SUSANTI, S., HEIJNEN, J.J., VAN LOOSDRECHT, M.C.M. (2002). Error diagnostics and data reconciliation for activated sludge modelling using mass balances. Wat. Sci. Tech., 45 (6), 145-156.

MENDOZA, D.F., PALACIO, L.M., GRACIANO, J.E.A., RIASCOS, C.A.M., VIANNA Jr., A.S., LE ROUX, G.A.C. (2013). Real-time optimization of an industrial-scale vapor recompression distillation process. Model validation and analysis. Ind. Eng. Chem. Res., 52 (16), 57355746.

MERCANGOZ, M., DOYLE, F.J. III (2008). Real-time optimization of the pulp mill benchmark problem. Comput. Chem. Eng., 32 (4-5), 789-804.

MERINO, A., ACEBES, L.F., MAZAEDA, R., DE PRADA, C. (2009). A Modelling and Simulation of the Sugar Production Process. Revista Iberoamericana de Automática e Informática Industrial, 6 (3), 21 -.

MOYSAN, J.M., HURONT, M.J., PARADOWSKI, H., VIDAL, J. (1983). Prediction of the solubility of hydrogen in hydrocarbon solvents through cubic equations of state. Chem. Eng. Sci., 38 (7), 1085-1092.

MUSKE, K.R., EDGAR, T.F. (1997). Nonlinear state estimation. Nonlinear Process Control. Henson, M.A., Seborg, D.E., Eds. Prentice Hall: New Jersey, 311-370.

NARASIMHAN, S., JORDACHE, C. (2000). Data reconciliation and gross error detection. Gulf Publishing Company, Houston, Texas.

NG, D.K.S., FOO, D.C.Y., TAN, R.R., EL-HALWAGI, M.M. (2010). Automatic targeting for concentration and property-based total resource conservation network. Comput. Chem. Eng., 34, 825-845.

NORGAARD, M. (2000). Neural Network Based System Identification Toolbox. Dept. Automation, Technical University of Denmark.

OGUNNAIKE, B.A., RAY, W.H. (1994). Process dynamics, modeling and control. Oxford University Press.

OlLERO DE CASTRO, P., FERNÁNDEZ CAMACHO, E. (1997). Control e Instrumentación de Procesos Químicos. Ed. Síntesis.

ÖZYURT, D.B., PIKE, R.W. (2004). Theory and practice of simultaneous data reconciliation and gross error detection for chemical processes. Comput. Chem. Eng., 28, 381-402.

PANTELIDES, C.C., RENFRO, J.G. (2013). The online use of first-principles models in process operations: Review, current status and future needs. Comput. Chem. Eng., 51, 136-148.

PERAMANU, S., COX, B.G., PRUDEN, B.B. (1999). Economics of hydrogen recovery processes for the purification of hydroprocessor purge and off-gases. Int. J. Hydrogen Energy, 24 (5), 405-424. 
POULINA, E., HODOUIN, D., LACHANCE, L. (2010). Impact of plant dynamics on the performance of steady-state data reconciliation. Comput. Chem. Eng., 34, 354-360.

QUELHAS, A.D., CASTRO DE JESUS, N.J., PINTO, J.C. (2013). Common vulnerabilities of RTO implementations in real chemical processes. Canadian J. Chem. Eng., 91 (4), 652-668.

QUESADA, I., GROSSMANN, I.E. (1995). Global optimization of bilinear process networks with multicomponent flows. Comput. Chem. Eng., 19 (12), 1219-1242.

REID, R.C., PRAUSNITZ, J.M., POLING, B.E. (1987). The Properties of Gases and Liquids, $4^{\text {th }}$ ed. McGraw-Hill.

RIAZI, M.R., ROOMI, Y.A. (2007). A method to predict solubility of hydrogen in hydrocarbons and their mixtures. Chem. Eng. Sci., 62, 6649-6658.

RICKER, N.L., LEE, J.H. (1995). Non-linear modeling and state estimation for the Tennessee Eastman challenge process. Comput. Chem. Eng., 19 (9), 983-1005.

RONZE, D., FONGARLAND, P., PITAULT, I., FORISSIER, M. (2002). Hydrogen solubility in straight run gasoil. Chem. Eng. Sci., 57, 547-553.

SARABIA, D., DE PRADA, C., GOMEZ, E., GUTIERREZ, G., CRISTEA, S., MENDEZ, C.A., SOLA, J.M., GONZALEZ, R. (2012). Data reconciliation and optimal management of hydrogen networks in a petrol refinery. Control Eng. Pract., 20 (4), 343-354.

SARDASHTI, M.R., BIRJANDI, SHAHRAKI, F. (2011). Off-gases optimization in a hydrogen network refinery. Chem. Eng. Technol., 34 (12), 1974-1982.

SERRALUNGA, F.J., AGUIRRE, P.A., MUSSATI, M.C. (2014). Including disjunctions in real-time optimization. Ind. Eng. Chem. Res., 53 (44), 17200-17213.

SERRALUNGA, F.J., MUSSATI, M.C., AGUIRRE, P.A. (2013). Model adaptation for real-time optimization in energy systems. Ind. Eng. Chem. Res., 52 (47), 16795-16810.

SILDIR, H., ARKUN, Y., CAKAL, B., GOKCE, D., KUZU, E. (2013). Plant-wide hierarchical optimization and control of an industrial hydrocracking process. J. Process Control, 23 (9), 1229-1240.

SILDIR, H., ARKUN, Y., CAKAL, B., GOKCE, D., KUZU, E. (2012). A dynamic non-isothermal model for a hydrocracking reactor: Model development by the method of continuous lumping and application to an industrial unit. J. Process Control, 22 (10), 1956-1965.

SKOGESTAD, S. (2000). Plantwide control: the search for the self-optimizing control structure. J. Process Control, 10, 487-507.

SKOGESTAD, S. (2004). Control structure design for complete chemical plants. Comput. Chem. Eng., 28 (1-2), 219-234. 
SODERSTROM, T.A., EDGAR, T.F., RUSSO, L.P., YOUNG, R.E. (2000). Industrial application of a large-scale dynamic data reconciliation strategy. Ind. Eng. Chem. Res., 39 (6), 16831693.

DE SOUZA, G., ODLOAK, D., ZANIN, A.C. (2010). Real time optimization (RTO) with model predictive control (MPC). Comput. Chem. Eng., 34 (12), 1999-2006.

STANLEY, G.M.; MAH, R.S.H. (1981). Observability and redundancy in process data. Chem. Eng. Sci., 36, 259-272.

STEFANIDIS, G.D., BELLOS, G.D., PAPAYANNAKOS, N.G. (2005). An improved weighted average reactor temperature estimation for simulation of adiabatic industrial hydrotreaters. Fuel Proc. Tech., 86, 1761-1775.

STEFANO, S., MANENTI, F., GROTTOLI, M.G., FABBRI, P., PIERUCCI, S. (2010). Sulfur recovery units: adaptive simulation and model validation on an industrial plant. Ind. Eng. Chem. Res., 49 (12), 5714-5724.

STEPHANOPOULOS, G. (1984). Chemical Process Control: An Introduction to Theory and Practice. Prentice-Hall.

TAMHANE, A.C., MAH, R.S.H. (1985). Data reconciliation and gross error detection in chemical process networks. Technometrics, 27 (4), 409-422.

TJOA, I.B., BIEGLER, L.T. (1992). Reduced successive quadratic programming strategy for errors-in-variables estimation. Comput. Chem. Eng., 16 (6), 523.

TOWLER, G.P., MANN, R., SERRIERE, A.J.-L., GABAUDE, C.M.D. (1996). Refinery hydrogen management: cost analysis of chemically-integrated facilities. Ind. Eng. Chem. Res., 35 (7), 2378-2388.

TSAMATSOULIS, D., PAPAYANNAKOS, N. (1998). Investigation of HDS kinetics, Chem. Eng. Sci., $53,3449-3458$.

ULLMANN'S Encyclopedia of Industrial Chemistry, $7^{\text {th }}$ ed.

VAN DEN HEEVER, S.A., GROSSMANN, I.E. (2003). A strategy for the integration of production planning and reactive scheduling in the optimization of a hydrogen supply network. Comput. Chem. Eng., 27, 1813-1839.

VEGA, P., REVOlLAR, S., FRANCISCO, M., MARTIN, J.M. (2014). Integration of set point optimization techniques into nonlinear MPC for improving the operation of WWTPs. Comput. Chem. Eng., 68, 78-95.

WANG, X., PALAZOGLU, A., EL-FARRA, N.H. (2015). Operational optimization and demand response of hybrid renewable energy systems. Applied Energy, 143, 324-335.

WARD, J.D., MELLICHAMP, D.A., DOHERTY, M.F. (2006). Insight from economically optimal steady-state operating policies for dynamic plantwide control. Ind. Eng. Chem. Res., 45 (4), 1343-1353. 
XENOS, D.P., CICCIOTTI, M., KOPANOS, G.M., BOUASWAIG, A.E.F., KAHRS, O., MARTINEZBOTAS, R., THORNHILL, N.F. (2015). Optimization of a network of compressors in parallel: Real time optimization (RTO) of compressors in chemical plants - An industrial case study. Applied Energy, 144, 51-63.

YE, L., CAO, Y., MA, X., SONG, Z. (2014). A novel hierarchical control structure with controlled variable adaptation. Ind. Eng. Chem. Res., 53 (38), 14695-14711.

ZAVALA, V.M., CONSTANTINESCU, E.M., KRAUSE, T., ANITESCU, M. (2009). On-line economic optimization of energy systems using weather forecast information. J. Process Control, 19 (10), 1725-1736.

ZHANG, Q., FENG, X., LIU, G., CHU, K.H. (2011). A novel graphical method for the integration of hydrogen distribution systems with purification reuse. Chem. Eng. Sci., 66, 797-809.

ZHANG, Z., CHEN, J. (2014). Simultaneous data reconciliation and gross error detection for dynamic systems using particle filter and measurement test. Comput. Chem. Eng., 69, 66-74.

ZHANG, Z., SHAO, Z., CHEN, J. (2015). Programming strategies of sequential incremental-scale subproblems for large scale data reconciliation and parameter estimation with multioperational conditions. Ind. Eng. Chem. Res., 54 (21), 5697-5709.

ZHANG, J., ZHU, X.X., TOWLER, G.P. (2001). A simultaneous optimization strategy for overall integration in refinery planning. Ind. Eng. Chem. Res., 40, 2640-2653.

ZHAO, Z.H., LIU, G.L., FENG, X. (2006). New graphical method for the integration of hydrogen distribution systems. Ind. Eng. Chem. Res., 45 (19), 6512-6517.

ZHAO, Z., LIU, G., FENG, X. (2007). The integration of the hydrogen distribution system with multiple impurities. Chem. Eng. Res. Des., 85 (A9), 1295-1304.

ZHOU, H., LU, J., CAO, Z., SHI, J., PAN, M., LI, W., JIANG, Q. (2011). Modeling and optimization of an industrial hydrocracking unit to improve the yield of diesel or kerosene. Fuel, 90, 3521-3530.

ZHOU, L., LIAO, Z., WANG, J., JIANG, B., Yang, Y. (2012). Hydrogen sulfide removal process embedded optimization of hydrogen network. Int. J. Hydrogen Energy, 37 (23), 1816318174.

ZHOU, L., LIAO, Z., WANG, J., JIANG, B., YANG, Y., HUI, D. (2013). Optimal design of sustainable hydrogen networks. Int. J. Hydrogen Energy, 38, 2937-2950. 



\section{ESTUDIO SOBRE EL MODELADO,} ReCONCILIACIÓN DE DATOS Y OPERACIÓN ÓPTIMA de Redes de Hidrógeno en Refinerías de Petróleo

El Anexo $A$ es un resumen en español de la tesis. El resumen está organizado de acuerdo con el siguiente esquema. En primer lugar se incluyen los objetivos y la motivación de la tesis, el estado del arte, una descripción del proceso, los principales resultados y publicaciones, y asimismo la metodología seguida en la consecución de dichos objetivos. A continuación se exponen los principales resultados y conclusiones del trabajo, ordenados de acuerdo con los siguientes temas que se corresponden con los distintos capítulos:

- desarrollo de un modelo simplificado de una unidad hidrodesulfuradora de gasóleo industrial y su validación con datos reales;

- $\quad$ aplicación de la técnica de optimización de control self-optimizing a dicha unidad hidrodesulfuradora, para discernir una estrategia automática eficiente de control que garantice una operación óptima desde el punto de vista del aporte de hidrógeno, considerando dicha planta aislada del conjunto de la red global;

- con el objetivo de la gestión óptima de la red de hidrógeno, desarrollo de modelos para las diferentes unidades que componen dicha red, justificación de las hipótesis admitidas, e implementación en EcosimPro ${ }^{\circledR}$;

- calibración en base a datos reales de dicho modelo de la red para distintos periodos de operación, por técnicas de reconciliación de datos mediante optimización;

- formulación del problema de toma de decisiones basada en modelo para la operación óptima en tiempo real de dicha red de hidrógeno, y análisis de soluciones;

- $\quad$ trabajo futuro.

\section{A.1- OBJETIVOS}

Se ha llevado a cabo un estudio sobre la gestión óptima en tiempo real de redes de hidrógeno en refinerías de petróleo, con referencia a la refinería de Petronor ${ }^{\circledR}$ perteneciente al grupo 
Repsol ${ }^{\circledR}$ y situada en Muskiz (Vizcaya). La tesis consiste en la aplicación de técnicas bien conocidas y establecidas como el modelado de procesos y la optimización a un tema interesante en la actualidad, como son las redes de hidrógeno en refinerías de petróleo. Los resultados obtenidos son coherentes y robustos, y las soluciones alcanzadas pueden ser directamente aplicadas en la práctica industrial.

El problema abordado tiene gran relevancia industrial, con el propósito general de mejorar la operación en tiempo real, ahorrar recursos en este caso materiales relativos al hidrógeno, y aumentar el conocimiento del sistema en la medida de lo posible. Asimismo se han desarrollado librerías en el entorno de simulación EcosimPro ${ }^{\circledR}$.

\section{A.2- MOTIVACIÓN}

Las refinerías de petróleo procesan hidrocarburos fósiles con el fin de producir combustibles bien para calefacción o para transporte, así como precursores para la industria petroquímica, consumiendo grandes cantidades de materias primas y energía. El ahorro de recursos materiales y energéticos es un asunto prioritario para las plantas de proceso en general y en particular para las refinerías de petróleo, debido a los bajos márgenes de beneficio y las enormes cantidades procesadas. El trabajo estudia la eficacia en el uso del hidrógeno como materia prima en la refinería de Petronor ${ }^{\circledR}$ (Muskiz, Vizcaya), perteneciente al grupo Repsol ${ }^{\circledR}$. El hidrógeno $\mathrm{H}_{2}$ es una materia prima cara que se emplea en muchos procesos en una refinería de petróleo, distribuyéndose por medio de una red de hidrógeno desde las plantas productoras hasta las plantas consumidoras. En las plantas consumidoras, el $\mathrm{H}_{2}$ se emplea principalmente como reactivo en reacciones de desulfuración, desnitrificación, y desaromatización de gasolina y gasóleo, en presencia de otras reacciones laterales consumidoras de $\mathrm{H}_{2}$. Las reacciones de desulfuración y desnitrificación permiten no generar emisiones de gases ácidos $\left(\mathrm{SO}_{\mathrm{x}}, \mathrm{NO}_{\mathrm{x}}\right)$, tanto en el uso de combustibles para calefacción como en los motores de combustión, evitando así la contaminación atmosférica.

En los últimos años, debido a la necesidad de procesar crudos cada vez más pesados por tanto con mayor contenido de azufre y otros contaminantes, y también debido a una legislación ambiental más restrictiva, las necesidades de $\mathrm{H}_{2}$ han aumentado de forma constante, de modo que el $\mathrm{H}_{2}$ ha cobrado un papel muy importante desde el punto de vista económico a nivel global de la refinería. Un uso eficaz del $\mathrm{H}_{2}$ en la operación diaria es deseable no sólo por su elevado coste de producción, sino también porque la penalización económica es incluso mayor en escenarios donde la capacidad de producción de $\mathrm{H}_{2}$ es cuello de botella para la capacidad de procesamiento de crudo. Por otra parte, las decisiones relativas a la gestión de la red de $\mathrm{H}_{2}$ son complejas dado que están involucradas muchas plantas y restricciones de operación, además con un alto grado de interacción y no sólo desde un punto de vista de la optimalidad, sino también desde un punto de vista práctico, porque se encargan varios operadores distribuidos en distintas salas de control. Por tanto, disponer de un criterio cuantitativo para la toma de decisiones puede ser muy útil. Además, el tipo de crudo cambia normalmente cada dos o tres días, y también ciertas especificaciones de los productos pueden cambiar de 
acuerdo con la gestión global de la refinería; como consecuencia, los escenarios relativos al consumo de $\mathrm{H}_{2}$ en las unidades consumidoras individuales pueden experimentar cambios significativos con cierta frecuencia, haciendo interesante el disponer de una herramienta orientada a la toma de decisiones para la optimización de la operación.

\section{A.3- ESTADO DEL ARTE}

La gestión óptima de redes de hidrógeno en refinerías de petróleo se ha abordado previamente por otros grupos de investigación, aunque bajo diferentes perspectivas:

- Diseño.

El grupo de investigación de la Universidad de Manchester ha trabajado en el tema durante muchos años, desarrollando la técnica del pinch de redes de $\mathrm{H}_{2}$ análoga a la técnica del pinch para redes de intercambiadores de calor.

Hasta donde sabemos, el modelo del reactor tiene en cuenta los mismos fenómenos que se consideran en este trabajo; sin embargo, aunque en la herramienta desarrollada en Manchester se emplean modelos más rigurosos y exactos para las relaciones de equilibrio termodinámico, la flexibilidad desde el punto de vista de la operación de la planta es limitada de acuerdo con el propósito de diseño: la pureza de $\mathrm{H}_{2}$ de todos los productores (incluyendo los separadores de alta presión) y de los gases de purga de todas las plantas consumidoras y todas las unidades de membranas se considera constante, es decir, las condiciones de operación son fijas en lo que respecta a la entrada del reactor y otras purezas de $\mathrm{H}_{2}$, de forma tal que las relaciones de equilibrio en los separadores pueden considerarse invariables. Por tanto, no se tiene en cuenta la variabilidad del proceso en tiempo real, siendo un enfoque adecuado para los propósitos de diseño perseguidos.

- Operación óptima en tiempo real.

Algunas compañías proveedoras de servicios como por ejemplo Inprocess ${ }^{\circledR}$ sita en Barcelona, ofrecen un paquete de simulación basado en el simulador de procesos comercialmente disponible Hysys ${ }^{\circledR}$ y sus funcionalidades de optimización, para determinar la operación óptima de redes de $\mathrm{H}_{2}$ de refinerías.

Hasta donde sabemos, a pesar de que el enfoque seguido en relación a las técnicas de optimización, las hipótesis asumidas y otras restricciones de proceso sea compartido e igualmente sistemático y riguroso, sin embargo un modelo flexible y cuya calibración sea susceptible de ser fácilmente actualizada puede ser valioso, lo cual se puede potenciar y realzar con un modelo dedicado ad hoc como el desarrollado en este trabajo. 
El marco teórico que sustenta este trabajo está bien establecido tanto en el ámbito del modelado de procesos basado en primeros principios físico-químicos como en el ámbito de la optimización con distintos fines, principalmente la mejora en la operación del proceso.

Las técnicas de optimización se emplean en la industria de procesos en diferentes campos: diseño, control predictivo de procesos multivariables, planificación. En la industria del refino de petróleo, se resuelven a diario problemas lineales LP de gran dimensión para tomar decisiones sobre la producción de acuerdo con los precios actuales del crudo y los distintos productos. Asimismo, se resuelven en tiempo real problemas LP de tamaño mediano en aplicaciones de MPC (model predictive control), especialmente en columnas de destilación debido a su gran contribución al consumo total de energía y también a la dificultad añadida de las interacciones y la naturaleza del control multivariable en dichas unidades.

\section{A.4- DESCRIPCIÓN DE LA RED DE HIDRÓGENO}

El hidrógeno $\mathrm{H}_{2}$ es un recurso caro que se emplea en muchos procesos en una refinería de petróleo, distribuyéndose mediante una red desde las unidades productoras hasta las unidades consumidoras.

La mayor parte de las plantas consumidoras son plantas de hidrotratamiento, donde el $\mathrm{H}_{2}$ se usa como reactivo para la desulfuración, desnitrificación y desaromatización de las gasolinas y gasóleos. Otro proceso importante donde se consume $\mathrm{H}_{2}$ son las unidades de hidrocraqueo catalítico, cuyos principales productos son gasóleo y keroseno. En lo que respecta a las plantas productoras, el $\mathrm{H}_{2}$ de alta pureza se genera en hornos de reformado catalítico de gas natural con vapor de agua, llamados en este caso H3 y H4. Membranas de tipo PSA a la salida permiten alcanzar elevadas purezas. Existen otras dos plantas productoras de hidrógeno, las unidades de platformado llamadas P1 y P2, no siendo verdaderamente variables de decisión en la operación de la red puesto que el $\mathrm{H}_{2}$ generado es un subproducto del proceso de reformado catalítico. El objetivo de las unidades de platformado es convertir gasolinas obtenidas del crudo por destilación y típicamente con valores bajos del índice de octano, en moléculas más complejas de tipo aromático con un mayor octanaje. En este proceso tienen lugar reacciones de deshidrogenación, y se generan cantidades importantes de $\mathrm{H}_{2}$ como subproducto. Este $\mathrm{H}_{2}$ tiene una baja pureza entorno al 0.65 to $0.83 \% 1 \mathrm{~mol}$, porque en muchas reacciones secundarias se producen hidrocarburos ligeros de menor valor como metano, etano, propano y butanos. En la Figura A.1 se muestran en rojo las plantas productoras de tipo horno de reformado a partir de gas natural y vapor, con los correspondientes colectores de distribución asociados; las plantas de platformado se muestran en lila, también con los correspondientes colectores de distribución asociados; mientras que en verde se muestran las plantas consumidoras, cuyos aportes frescos de $\mathrm{H}_{2}$ proceden en general de varios colectores. 


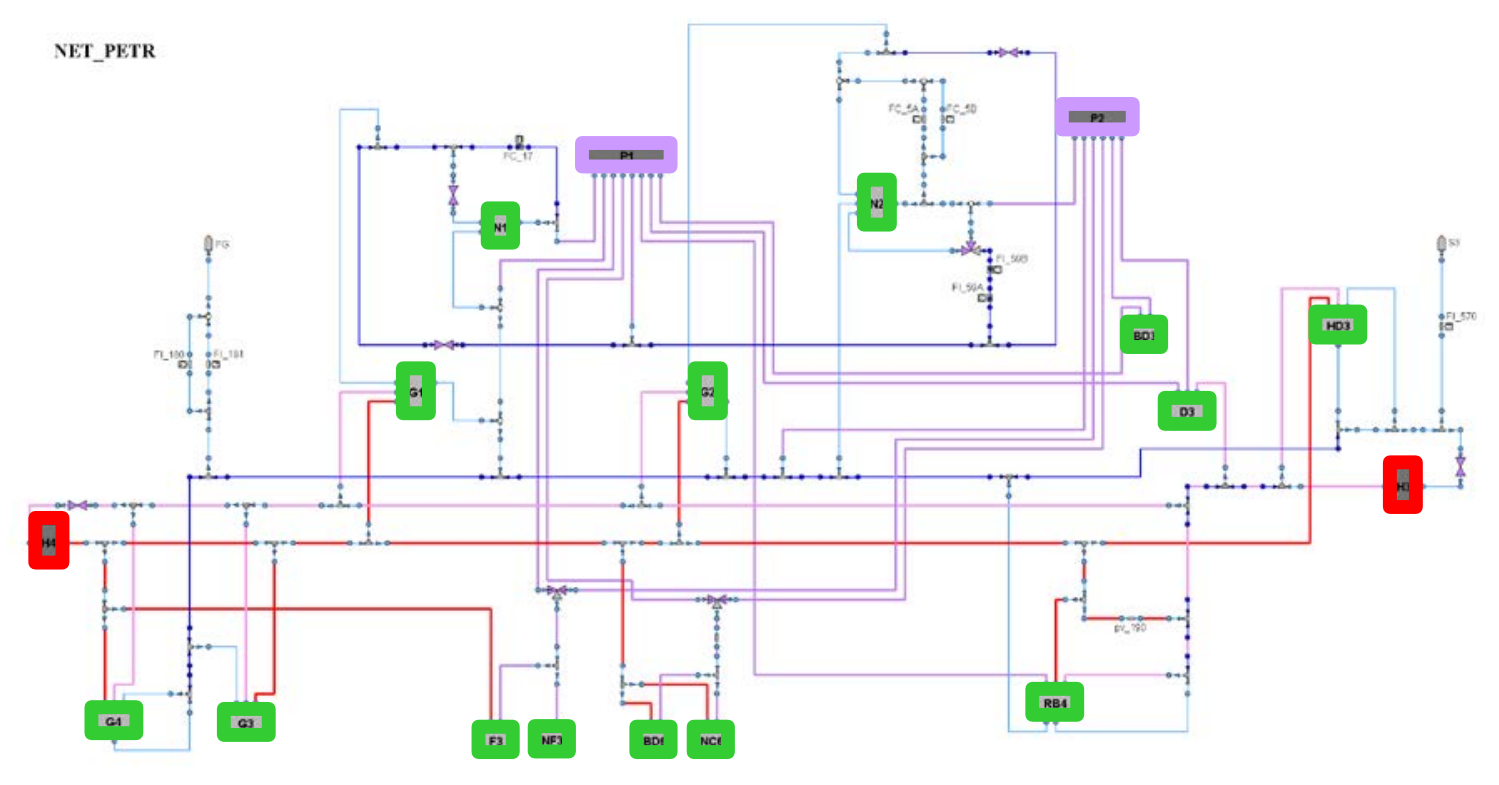

Figura A.1. Esquema de la red de $\mathrm{H}_{2}$ de la refinería Petronor ${ }^{\circledR}$.

\section{A.4.1- Descripción de una planta hidrodesulfuradora HDS general}

En una planta consumidora general, Figura A.2, la alimentación de hidrocarburo $(H C)$ se mezcla a la entrada del reactor $(\Delta R)$ con una corriente recirculada $(R)$ de $\mathrm{H}_{2}$ y con una corriente de aporte fresco de $\mathrm{H}_{2}$ procedente de la red: típicamente desde los colectores ligados a las dos plantas productoras $\mathrm{H} 4$ y $\mathrm{H} 3$ y también desde el colector de baja pureza $\mathrm{LPH}$ que recoge los excesos de otras plantas consumidoras y de las unidades de platformado. El $\mathrm{H}_{2}$ no reaccionado a la salida del reactor es separado en una cámara de separación a alta presión HP (HPsep); la mayor parte del mismo se recircula a la entrada $(R)$, y otra parte se puede purgar a otro colector de baja pureza $\left(L P H_{H P}\right)$. Normalmente es necesaria una purga en el sistema de alta presión HP para mantener la restricción de mínima pureza en el sistema de HP, evitando la acumulación de gases ligeros. Los gases ligeros (metano y etano fundamentalmente y en menor proporción propano y otros gases) proceden tanto de la generación en el reactor como del aporte fresco desde fuentes de baja pureza.

El azufre reaccionado se convierte en sulfuro de hidrógeno $\mathrm{H}_{2} \mathrm{~S}$, que se elimina mediante absorción en una solución de amina. Aguas abajo del separador HP de alta presión, distintas operaciones de separación a baja presión tanto de una sola etapa como columnas de destilación permiten una separación completa del $\mathrm{H}_{2}$ y los gases ligeros de la corriente líquida de hidrocarburo desulfurado. Estas corrientes de gas procedentes de operaciones de separación (LPoper) a media y baja presión MP/LP son quemadas como fuel gas ( $\left.F G_{L P}\right)$, ya que su pureza de $\mathrm{H}_{2}$ no es lo suficientemente alta como para hacer rentable su recuperación o reutilización. En lo que respecta a las purgas de los sistemas de MP/LP, estos caudales no son grados de libertad manipulables en la operación, siendo separados bajo control de presión con el propósito de una completa separación del gas disuelto en el hidrocarburo líquido. Las purgas del sistema HP son de diferente naturaleza: estas purgas son generalmente variables de 
decisión manipuladas, normalmente enviadas a colectores de baja pureza LPH debido a su relativamente alta pureza de $\mathrm{H}_{2}$, y por tanto pudiendo ser aprovechadas como aporte en otras plantas consumidoras.

En ciertas plantas se dispone además de una unidad de membranas $(Z)$, que hace posible la purificación y reciclo de una corriente de permeado $\left(F_{P R M} z\right)$ de mayor pureza que la entrada, a expensas de una corriente de purga a fuel gas $\left(F G_{z}\right)$ con menor pureza de $\mathrm{H}_{2}$.

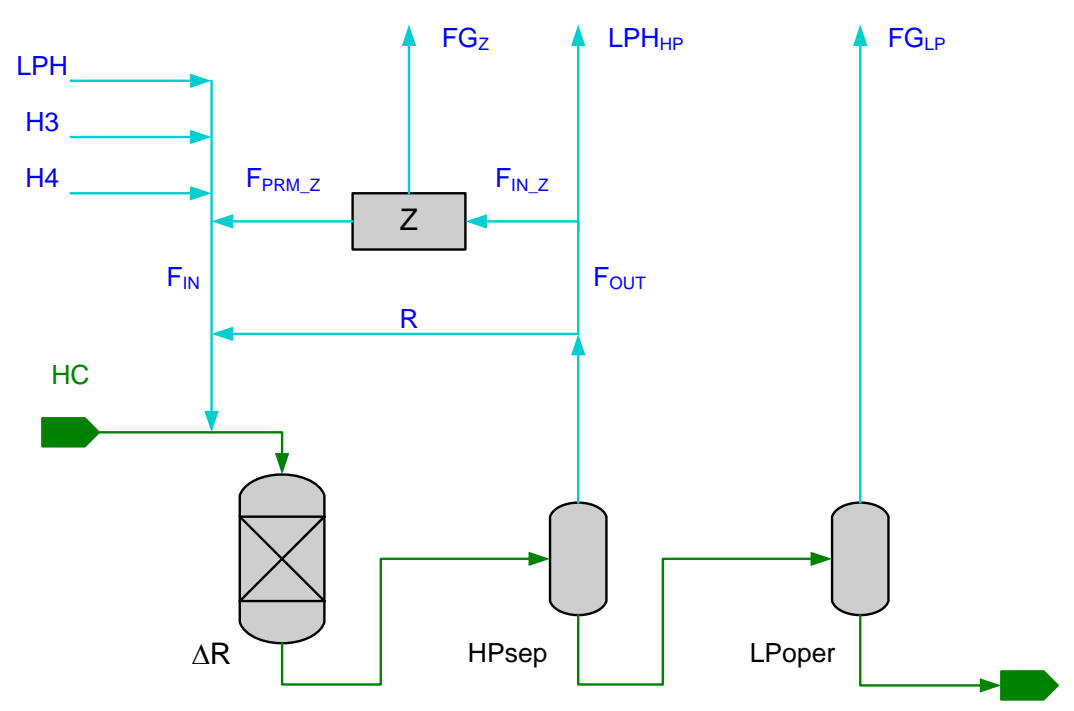

Figura A.2. Esquema de una planta consumidora de $\mathrm{H}_{2}$, con aporte fresco desde dos colectores de alta pureza ligados a plantas productoras $(\mathrm{H} 3, \mathrm{H} 4)$ y desde un colector de baja pureza (LPH).

\section{A.4.2- Gestión óptima de la red de hidrógeno}

La pureza de $\mathrm{H}_{2}$ ( $y, \% 1$ fracción molar) de las corrientes de gas empleadas como aporte fresco de las distintas plantas consumidoras varía típicamente entre 0.65 y $1 \% 1$ mol. El gas total $(F$, $\mathrm{Nm}^{3} / \mathrm{h}$ ) se usa como sinónimo de $\mathrm{H}_{2}$ no puro, siendo los ligeros o impurezas que acompañan al hidrógeno en las corrientes de gas principalmente metano, etano y en menor proporción propano y otros gases más pesados. La pureza de $\mathrm{H}_{2}$ del aporte fresco total puede variar dependiendo de la proporción mezclada de las entradas desde las distintas plantas productoras (H3, H4, P1, P2) o colectores de distribución (colector de baja pureza LPH), siendo la más barata tanto los excesos purgados en alta presión HP en otras plantas consumidoras como las corrientes procedentes de las plantas de platformado. Cuanto mayor es la pureza de $\mathrm{H}_{2}$, menor es el caudal de gas necesario como aporte fresco; sin embargo, en general, cuanto mayor es la pureza de $\mathrm{H}_{2}$ mayor es el coste de producción. Por tanto, surge un compromiso en lo que se refiere a eficacia: la eficacia aumenta con $\mathrm{H}_{2}$ de aporte fresco de alta pureza cuando no se tiene en cuenta el coste de producción, pero cuando sí se tiene en cuenta la relación puede ser no monótona.

La eficacia en el uso del $\mathrm{H}_{2}$ como un recurso material se considera de forma separada del proceso de tratamiento de hidrocarburo. Se asume la hipótesis de que la operación del reactor en lo que respecta a desulfuración, desnitrificación, desaromatización o hidrocraqueo 
permanece invariable con independencia de la redistribución global de caudales de $\mathrm{H}_{2}$, lo cual es razonable dado que siempre se mantiene una pureza mínima de $\mathrm{H}_{2}$ en el gas de salida del separador $\mathrm{HP}$, lo cual significa que el $\mathrm{H}_{2}$ está presente en las reacciones en suficiente exceso y pueden suponerse cinéticas de orden cero respecto del $\mathrm{H}_{2}$.

La eficacia en la gestión del $\mathrm{H}_{2}$ tiene más sentido a nivel de la red global de la refinería (Fig. 1.5), cuando se consideran todas las plantas productoras y consumidoras así como todos los colectores de distribución y tuberías de unión disponibles. El enfoque para abordar el problema de la gestión óptima se encuentra dirigido por un marco operacional en el que la producción de $\mathrm{H}_{2}$ debe siempre exceder al consumo de $\mathrm{H}_{2}$, puesto que el déficit de $\mathrm{H}_{2}$ es muy perjudicial para los catalizadores que son muy caros. Como la acumulación de $\mathrm{H}_{2}$ en un recipiente pulmón no es posible, y además la dinámica en la producción de $\mathrm{H}_{2}$ en los hornos de reformado de gas natural con vapor es lenta, el objetivo de una minimización de la producción de $\mathrm{H}_{2}$ ha de conseguirse por medio de:

- Un buen ajuste dinámico de la producción de $\mathrm{H}_{2}$ al consumo, con el objetivo de minimizar los excesos enviados a Fuel Gas bajo control de presión en los colectores. Éste es un problema de controlabilidad, abordado por el Dpto. de Control Avanzado de Petronor ${ }^{\circledR}$ en cooperación con el Dpto. de Producción mediante un controlador predictivo multivariable MPC ya diseñado y puesto en funcionamiento. El propósito es controlar ciertas especificaciones determinantes, por tanto evitando/reduciendo los márgenes previstos ante perturbaciones. El MPC tiene en cuenta las interrelaciones más significativas en el control multivariable del LPH, en el que se vierten excesos de las consumidoras y que a la vez sirve de aporte fresco a otras consumidoras.

- Una mejor redistribución del $\mathrm{H}_{2}$ desde las plantas productoras a las consumidoras. Este problema es de una naturaleza diferente, donde las actuaciones han de tomarse en una capa superior de la jerarquía y en una escala de tiempos más lenta que las relacionadas con el mencionado MPC. El problema se aborda con un enfoque de optimización en tiempo real RTO, y las decisiones a tomar para la gestión óptima de la red son:

- Qué plantas han de producir $\mathrm{H}_{2}$ y cuánto;

- Cuál es la combinación de caudales de aporte fresco desde cada colector para suministrar $\mathrm{H}_{2}$ a cada planta consumidora;

- Cuál ha de ser el punto de operación de las unidades de membranas y de otras purgas de alta presión HP.

Éste es el problema abordado en este trabajo. Se ha propuesto un modelo simplificado para las unidades involucradas, las hipótesis realizadas se han justificado, y el estado actual de la red se ha estimado mediante la resolución de un problema de reconciliación de medidas por técnicas de optimización, aprovechando toda la redundancia de medidas disponibles online. 


\section{A.5- PRODUCTO}

Los resultados de la tesis comprenden:

- Una simulación de la red de hidrógeno industrial en el entorno EcosimPro ${ }^{\circledR}$, con los mismos grados de libertad y variables manipuladas que las existentes físicamente en el proceso. Asimismo, implementaciones en EcosimPro ${ }^{\circledR}$ de los problemas de optimización para resolver la reconciliación de datos y la redistribución óptima, mediante el algoritmo resolvedor Snopt ${ }^{\circledR}$;

- Una librería desarrollada en EcosimPro ${ }^{\circledR}$ para el modelado de redes de hidrógeno, que puede ser fácilmente configurada y parametrizada. La librería además incorpora funcionalidades adicionales que permiten la generación semiautomática del código necesario para resolver los problemas de optimización;

- Un estudio de colaboración sobre posibles modificaciones en la topología de la red de hidrógeno de Petronor ${ }^{\circledR}$, liderado por el Centro de Tecnología de Repsol ${ }^{\circledR}$ en Móstoles (Madrid), desde septiembre de 2013 hasta julio de 2014. Los resultados finales se resolvieron y entregaron a partir de simulaciones y optimizaciones de la red de hidrógeno desarrolladas en EcosimPro ${ }^{\circledR}$ mediante de dicha librería;

- El problema de la gestión óptima de la red de hidrógeno -abarcando el modelo, la simulación y la implementación de los problemas de optimización- se está utilizando como caso de estudio en el proyecto europeo MORE "Real-time Monitoring and Optimization of Resource Efficiency in Integrated Processing Plants", desde noviembre de 2013 hasta noviembre de 2016, con el objetivo de monitorizar en tiempo real la eficacia en el uso de los recursos materiales y energéticos, orientada a una mejor gestión, y liderado por Prof. Sebastian Engell.

\section{A.6- PUBLICACIONES}

Se están preparando publicaciones para distintas revistas:

"Data reconciliation and optimal management of hydrogen networks in a petrol refinery", 2012. Sarabia Ortiz, D., de Prada Moraga, C., Gómez Sayalero, E., Gutiérrez Rodríguez, G., Podar Cristea, S., Sola Sáez, J.M. et al. Control Eng. Pract., 20 (4), 343-354.

Las publicaciones en conferencias internacionales se detallan a continuación:

"Grey-box modelling of an industrial hydrodesulphurization process", 2008. Gómez, E., de Prada, C., Sarabia, D., Méndez, C.A., Cristea, S., Sola, J.M., Unzueta, E. $18^{\text {th }}$ European Symposium on Computer Aided Process Engineering - ESCAPE 18. Lyon, 2008. Editors Bertrand Braunschweig and Xavier Joulia. Elsevier B.V./Ltd. ISBN 978-0-444-53228-2. 
"Optimal management of hydrogen supply and consumption networks of refinery operations", 2008. Méndez, C.A., Gómez, E., Sarabia, D., Cerdá, J., de Prada, C., Sola, J.M., Unzueta, E. $18^{\text {th }}$ European Symposium on Computer Aided Process Engineering - ESCAPE 18. Lyon. Editors Bertrand Braunschweig and Xavier Joulia. Elsevier B.V./Ltd. ISBN 978-0444-53228-2.

"Gestión óptima de redes de hidrógeno de refinerías", 2008. Gómez, E., de Prada, C., Sarabia, D., Méndez, C.A., Cristea, S., Sola, J.M., Unzueta, E. XXIX Jornadas de Automática, Tarragona.

"Grey-box modelling of an industrial hydrodesulphurization process", 2008. Gómez, E., de Prada, C., Sarabia, D., Méndez, C.A., Cristea, S., Sola, J.M., Unzueta, E. Reducit Workshop on Model Reduction for Industrial Control and Optimization Applications, 45 noviembre 2008, Frankfurt.

"Data reconciliation and optimal management of hydrogen networks of a real refinery", 2009. Sarabia, D., Cristea, S., Gómez, E., Gutiérrez, G., Méndez, C.A., Sola, J.M., de Prada, C.. Proceedings of Adchem International Symposium on Advanced Control of Chemical Processes. Koc, Turkey.

"Simplified modelling and validation of an industrial diesel hydrodesulfurization plant", 2010. Gómez, E., Sarabia, D., Cristea, S., Gutiérrez, G., Méndez, C.A., Sola, J.M., Unzueta, E., González, R., de Prada, C. DYCOPS 9th International Symposium on Dynamics and Control of Process Systems. Lovaina, Bélgica, 5- 7 julio 2010.

"Self-optimizing control for hydrogen optimization in a diesel hydrodesulfurization plant", 2012. Sayalero, E.G., Skogestad, S., de Prada, C., Sola, J.M., González, R. PSE 11th International Symposium on Process Systems Engineering. Singapore, Singapore. July 15-19 2012. (Oral presentation).

"Optimal operation of a petrol refinery hydrogen network", 2014. E. Gómez, G. Gutiérrez, D. Sarabia, C. de Prada, S. Mármol, J.M. Sola, R. González. IFORS 20th Conference of the International Federation of Operational Research Societies. Barcelona, Spain, July 1318, 2014. (Oral presentation).

"Resource efficiency indicators applied to refinery hydrogen networks", 2015. E. Gómez, G. Gutiérrez, D. Sarabia, S. Mármol, J.M. Sola, C. Pascual, R. González, C. de Prada. ECCE 10th European Congress of Chemical Engineering. Nice, France, September 27thOctober 1st, 2015.

$\underline{\text { Premio }}$

Premio Empresarios Agrupados E.A. Internacional 2008 al mejor trabajo de desarrollo de modelos y/o librerías en el entorno de modelado y simulación EcosimPro ${ }^{\circledR}$ : 
"Gestión óptima de redes de hidrógeno de refinerías", 2008. Gómez, E., de Prada, C., Sarabia, D., Méndez, C.A., Cristea, S., Sola, J.M., Unzueta, E. XXIX Jornadas de Automática, Tarragona, Spain, september 3-5, 2008. www.ecosimpro.com.

\section{A.7- METOdología}

El problema ha sido abordado siguiendo una secuencia lógica, con el objetivo final de disponer de una herramienta para la toma de decisiones basada en modelo que contribuya a una gestión más eficiente y óptima de la operación de la red en tiempo real.

En primer lugar se ha estudiado y comprendido el problema de la red de hidrógeno, incluyendo el análisis de datos experimentales, la revisión bibliográfica, y conversaciones con los operadores y técnicos de proceso. En segundo lugar el problema se ha formulado matemáticamente, por medio de un modelo basado en principios físico-químicos y leyes de conservación. Como paso previo a la reconciliación de datos automática mediante técnicas de optimización, se ha realizado una calibración manual detallada en simulación para tres escenarios diferentes con el propósito de ajustar valores por defecto para los parámetros del modelo, en particular los coeficientes del modelo de solubilidad, así como también aumentar el conocimiento del sistema con respecto a sensibilidad de variables y compromisos en el ajuste. A continuación, dicho modelo ha sido calibrado a partir de datos experimentales de la planta real, resolviendo un problema de reconciliación de datos a partir de técnicas de optimización, minimizando una función de error formada por las desviaciones modelomedidas. Una vez estimado el estado del proceso y los valores de todas las variables y parámetros a partir de la información disponible en línea, se ha planteado el problema de operación óptima en tiempo real con base al mismo modelo previamente calibrado, justificando todas las hipótesis realizadas, y resuelto igualmente mediante técnicas de optimización a través de la minimización de una función de coste de operación, sujeto a las restricciones de operación correspondientes con los límites adecuados de acuerdo con datos históricos o especificaciones del proceso.

Los principales resultados se muestran a continuación ordenados por epígrafes.

\section{A.8- RESULTADOS}

\section{A.8.1- MODELO SIMPLIFICADO Y VALIDACIÓN DE UNA PLANTA HIDRODESULFURADORA DE GASÓLEO INDUSTRIAL}

Se ha desarrollado un modelo dinámico simplificado de una unidad hidrodesulfuradora de gasóleo industrial, y se ha validado frente a datos reales. El objetivo es la predicción del 
consumo de hidrógeno en función de la carga de hidrocarburo, con el fin de conocer mejor la operación del proceso y asimismo evaluar su integración potencial en un sistema de ayuda a la decisión orientado a la operación óptima en tiempo real de la red de hidrógeno de la refinería. Se ha propuesto un modelo que combina primeros principios físico-químicos con elementos de caja negra. Una unidad hidrodesulfuradora es una de las principales plantas consumidoras de hidrógeno en una refinería. Su propósito es eliminar el azufre y otros contaminantes de una corriente de hidrocarburo. Debido al tamaño del problema y a la escala en la que opera, no son adecuados los modelos dinámicos detallados.

\section{A.8.1.1- Estado del Arte}

Distintos autores han reportado modelos matemáticos basados en primeros principios para modelar el comportamiento de reactores de planta piloto (Chowdhury et al., 2002), (Froment et al., 1994), (Korsten and Hoffmann, 1996), (Tsamatsoulis and Papayannakos, 1998), siendo modelos estáticos. Algunos autores hacen el esfuerzo de desarrollar modelos matemático rigurosos que tienen en cuenta todas las principales reacciones y simulan tanto el comportamiento de reactores a escala industrial y a escala de planta piloto (Bhaskar et al., 2004), (Bellos, Papayannakos et al., 2005), pero sólo unos pocos son dinámicos (Mederos, Ancheyta et al., 2006).

Con independencia de que el reactor sea el núcleo del proceso, se debe considerar un modelo de planta completa porque la operación en el reactor es consecuencia de otras variables de decisión y restricciones en distintas unidades de la planta. Sin embargo, que sepamos, no se ha publicado un modelo simplificado de planta completa para un proceso de hidrodesulfuración, con los mismos grados de libertad y variables medidas que en la planta real. Igualmente, las validaciones industriales publicadas están limitadas a períodos cortos de operación, no haciendo referencia a períodos grandes como meses.

Con el fin de conseguir un equilibrio entre simplicidad numérica y exactitud, se ha propuesto un modelo gris, que combina primeros principios físico-químicos con "pseudo" parámetros cinéticos que son estimados mediante una red neuronal. El modelo es válido desde el punto de vista de la predicción ya que captura el comportamiento inherente del sistema. Contribuciones previas al área de los modelos grises (Georgieva, Meireles, Feyo d'Azevedo, 2003), (Laurent, Boyer, Gatina, 2000), (Chen, Bernard, Bastin, Angelov, 2000) han demostrado la factibilidad del enfoque propuesto. 


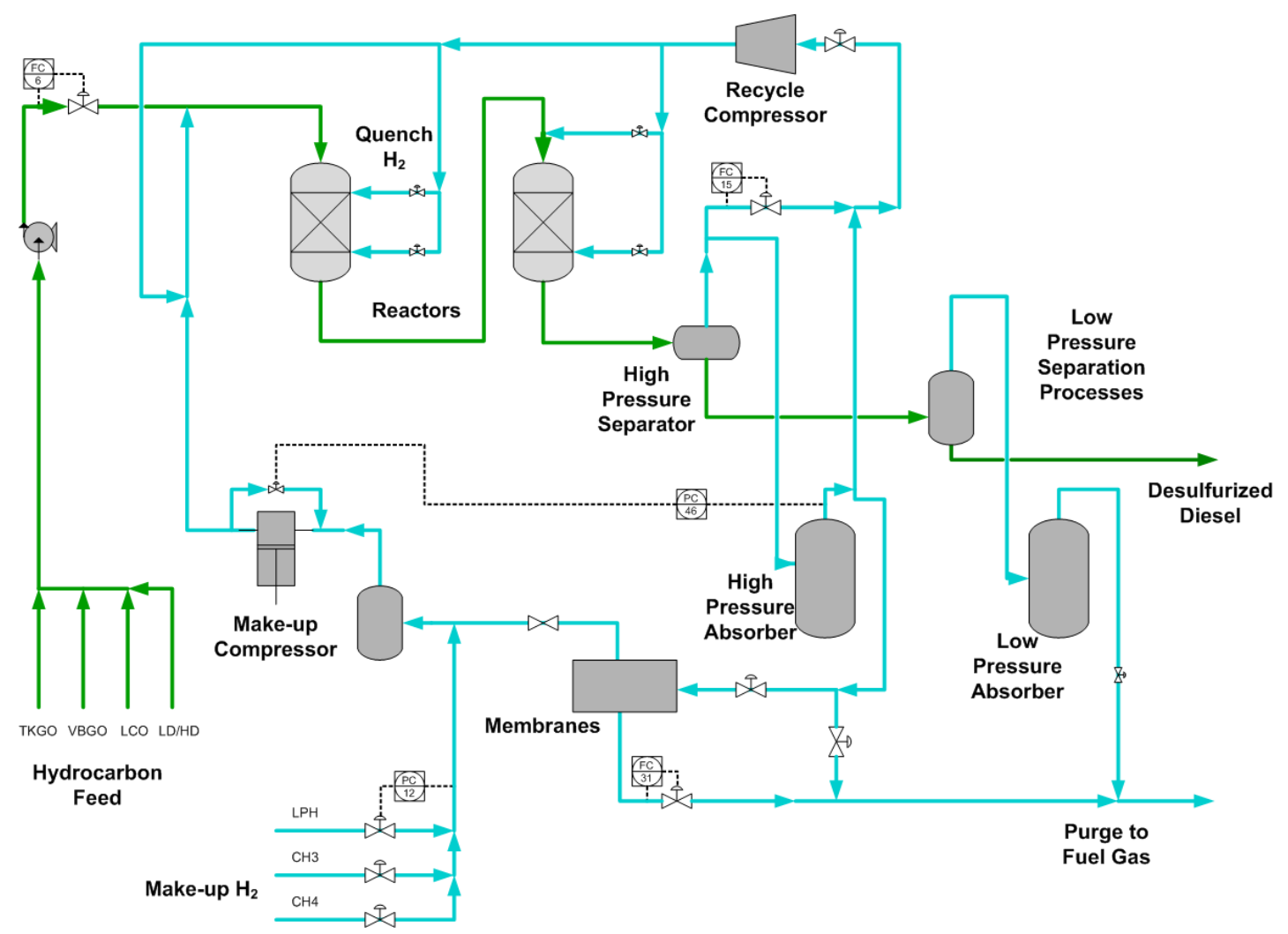

Figura A.3. Diagrama de flujo simplificado de una planta hidrodesulfuradora con membranas.

\section{A.8.1.2- Modelo y Validación}

Únicamente las unidades relevantes desde el punto de vista del hidrógeno $\mathrm{H}_{2}$ se han tenido en cuenta en el modelo: reactor, separador de alta presión HP, membranas, y englobadas en un único elemento todas las operaciones de separación aguas abajo del separador de alta presión HP. Para estas unidades se han desarrollado modelos de balances de material y de cantidad de movimiento. No se han considerado balances de energía debido a que la temperatura se controla en la entrada del reactor, también entre los lechos del catalizador con el $\mathrm{H}_{2}$ de quench, así como a la entrada del separador HP, por lo que se espera que no influyan de forma significativa en el consumo de hidrógeno y en la separación. Los balances de energía pueden proporcionar información acerca de la extensión de las diferentes reacciones, pero a la vez introducen mayor complejidad debido a un mayor número de parámetros a estimar (calores de reacción y calores específicos), por lo que se ha optado por especificar la temperatura como una condición de contorno manipulada.

Los cientos de componentes en la alimentación se han reducido a cuatro, con el criterio de ser los más importantes desde el punto de vista del consumo de $\mathrm{H}_{2}$ : hidrógeno, ligeros que acompañan al $\mathrm{H}_{2}$ en las corrientes de gas (principalmente metano y etano), azufre e hidrocarburo, englobando al resto de componentes presentes en la alimentación líquida.

En lo que respecta al reactor, un modelo detallado de primeros principios físico-químicos es demasiado complejo para el propósito de optimización en tiempo real, unido a la falta de 
medidas disponibles en línea. En el enfoque de modelado gris seguido, se han combinado los balances de materia básicos con elementos de caja negra que evitan descripciones complejas y detalladas de las relaciones constitutivas del proceso. En particular, las reacciones consideradas son: i) la eliminación de azufre; ii) el consumo de $\mathrm{H}_{2}$ debido a la desulfuración y el consumo de $\mathrm{H}_{2}$ en el resto de reacciones laterales; iii) la generación de gases ligeros. La reacción de hidrodesulfuración se ha modelado de primer orden con respecto de la concentración de azufre, y todas ellas de orden cero con respecto al $\mathrm{H}_{2}$, suponiendo que está presente en exceso en la fase líquida. Los tres coeficientes cinéticos que permiten calcular las velocidades de reacción, $k_{\mathrm{H} 2}{ }^{r}, k_{s}{ }^{r}, k_{\text {lig }}{ }^{r}$, se han obtenido a partir de una red neuronal (NN) en función de las principales variables del proceso: temperatura en el reactor, presión parcial de $\mathrm{H}_{2}$, y tipo de hidrocarburo alimentado: diésel de destilación directa, aceite cíclico ligero del FCC, diésel de la unidad de visbreaker, o diésel de tanques, siendo los regresores de la NN las proporciones de cada flujo respecto del total.

Para la estimación de parámetros se ha seguido un enfoque secuencial. En primer lugar, suponiendo que la dinámica en el establecimiento de flujos y presiones es despreciable comparada con la dinámica en composiciones, aquellos parámetros relacionados con los balances de cantidad de movimiento se han estimado con independencia del resto del modelo, puesto que se dispone de suficientes medidas de presión y caudal. La correlación de predicción de la pureza del permeado en la unidad de membranas también se obtuvo en esta etapa. En segundo lugar, se resolvió el problema inverso estacionario, fijando como entradas variables de respuesta medidas del sistema: pureza de $\mathrm{H}_{2}$ en la corriente de reciclo, concentración de azufre en la corriente de gasóleo desulfurado, y flujo de aporte fresco de $\mathrm{H}_{2}$. Esto permite obtener los tres coeficientes cinéticos, $k_{S}{ }^{r}, k_{\mathrm{H}^{2}}{ }^{r}, k_{\text {lig }}{ }^{r}, \mathrm{y}$ así después entrenar tres redes neuronales independientes.

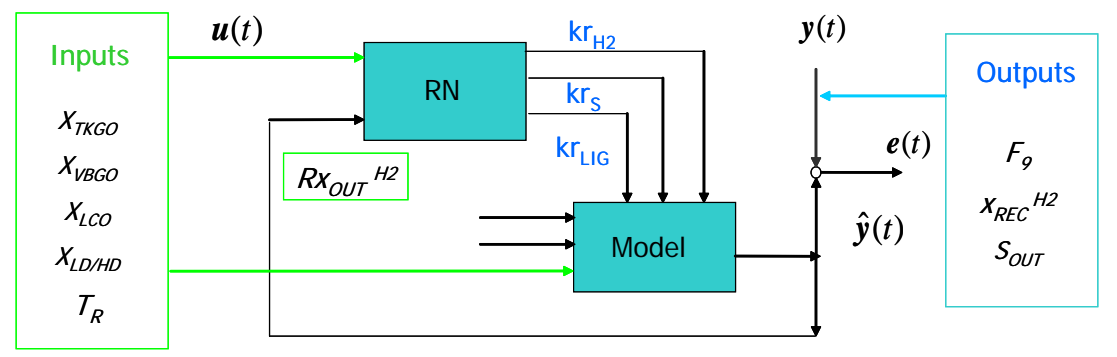

Figura A.4. Estructura para la estimación dinámica de parámetros.

En las siguientes Fig. se muestran datos de validación correspondientes a un mes de operación, donde la línea azul representa el modelo y la verde los datos experimentales. La salida más interesante del modelo, el caudal de aporte fresco desde el colector de baja pureza, se estima con menos de un $10 \%$ de error, y también se obtienen resultados razonables para la concentración de azufre en la corriente de gasóleo desulfurada. Es importante señalar que ni la composición de azufre en la alimentación ni la pureza de $\mathrm{H}_{2}$ del colector de baja pureza se miden, por lo que tampoco pueden esperarse resultados perfectos. Se han obtenido resultados similares para otros períodos de operación. 


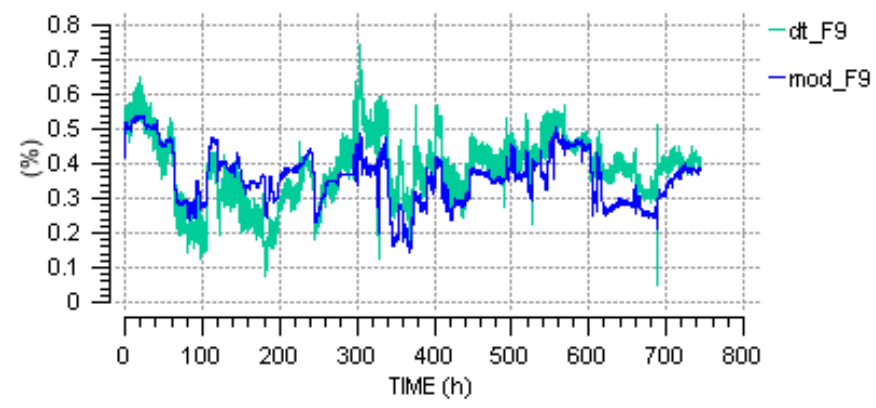

Figura A.5. Hidrógeno de make-up desde el colector de baja pureza LPH (entrada bajo regulación de presión).

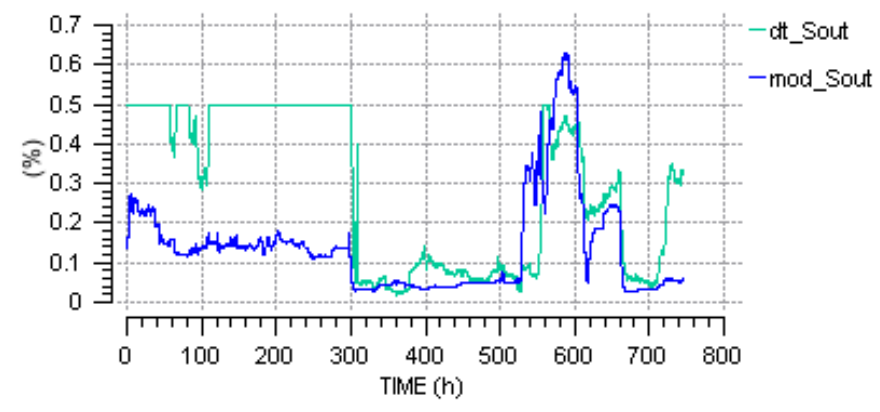

Figura A.6. Concentración de azufre en la corriente de gasóleo desulfurado (analizador fuera de servicio para 0-300 h).

\section{A.8.1.3- Conclusiones}

Se ha propuesto un modelo dinámico simplificado para una unidad hidrodesulfuradora de gasóleo HDS industrial y se ha validado frente a datos reales. A pesar de la sencillez, las predicciones de consumo de $\mathrm{H}_{2}$ concuerdan bien con los datos experimentales en las condiciones estudiadas, mientras que las predicciones para la concentración de azufre de salida son menos exactas.

A pesar de que habría que mejorarlo antes de poder utilizarlo como parte de una herramienta de ayuda a la decisión, el modelo tiene dos potenciales aplicaciones. La primera es la predicción del consumo de hidrógeno en función de la carga de hidrocarburo, con el fin de la toma de decisiones óptimas sobre producción y redistribución de $\mathrm{H}_{2}$ a nivel de la red global. El segundo es la optimización de la operación interna de la planta hidrodesulfuradora, en concreto del grado de severidad en la operación en el reactor (temperatura) para cumplir la especificación de desulfuración con el mínimo margen respecto del límite, es decir, de la forma más eficaz. La severidad en la operación es manipulada por el operador con el fin de conseguir el grado deseado de desulfuración, siendo la concentración de azufre en el hidrocarburo de salida el objetivo del proceso. Dicha severidad influye en el consumo de hidrógeno y también contribuye a la desactivación del catalizador, de modo que es deseable operar el reactor a la mínima temperatura posible ya que eso es beneficioso en ambos aspectos. 


\section{A.8.2- CONTROL SELF-OPTIMIZING PARA LA GESTIÓN ÓPTIMA DE HIDRÓGENO APLICADO A UNA PLANTA HIDRODESULFURADORA}

El objetivo es aplicar la técnica de control self-optimizing (Skogestad, 2000) para averiguar una potencial estructura de control de planta completa orientada a la optimización del consumo de hidrógeno en una planta hidrodesulfuradora de gasóleo de una refinería de petróleo.

La determinación de una buena estructura de control de planta completa es un asunto de gran importancia práctica para lograr una operación óptima. El procedimiento sistemático de Skogestad busca una estrategia de control que pueda implementarse de una manera sencilla y robusta, garantizando una operación óptima o cerca de la optimalidad la mayor parte del tiempo. En primer lugar, se controlan aquellas variables que son restricciones activas del problema, porque suelen ser aquellas con mayor penalización económica en caso de alejamiento; después, para los restantes grados de libertad desde el punto de vista de la optimización económica, se buscan variables self-optimizing. Se llama control self-optimizing (Skogestad, 2000) a lograr una operación óptima desde el punto de vista económico o próxima al óptimo mediante una política de consignas constantes para los reguladores. De este modo se evita, o al menos se reduce, la necesidad de una capa superior de optimización RTO, siendo todavía el control feedback la forma más sencilla de implementar una estrategia de control en la práctica industrial (Skogestad, 2004). La técnica de control self-optimizing se ha aplicado satisfactoriamente a muchos procesos, por ejemplo Araujo, Govatsmark and Skogestad (2007); Lid and Skogestad (2008).

\section{A.8.2.1- Aplicación sistemática del método de control self-optimizing}

El modelo de la planta hidrodesulfuradora utilizado es el desarrollado en el capítulo 2. A continuación se aplica el procedimiento sistemático para el control de planta completa (Skogestad, 2004) con el fin de determinar la estructura de control self-optimizing, siguiendo los pasos del análisis económico en estado estacionario.

Se dispone de un total de 5 grados de libertad para la optimización económica de acuerdo con el modelo del proceso. Las siguientes son las variables manipuladas $(u)$ naturales en la operación:

- Caudal de $\mathrm{H}_{2}$ de aporte fresco de las plantas productoras $F_{H 3}, F_{H 4}$;

- Caudal de entrada a la unidad de membranas $F_{I N \_} z$

- Proporción de caudal purgado en membranas respecto de la entrada $F_{F G_{-} Z} / F_{I N_{-} Z} ;$

- Caudal de purga directa a fuel gas desde el sistema HP de alta presión $F_{F G_{-} H P \text {. }}$

Desde el punto de vista del estado de la planta, las perturbaciones relativas tanto a la alimentación de hidrocarburo como a las condiciones de operación del proceso tienen influencia sobre los distintos valores de gas separado en las operaciones de separación a baja presión $F_{F G_{-} L P,} Y_{F G_{-} L P}{ }^{H 2}$, así como en el consumo de hidrógeno $\Delta R_{H 2}$ y la generación de ligeros 
$\Delta R_{L I G}$ en el reactor. Para el análisis según futuros escenarios, las perturbaciones externas que merecen ser tenidas en cuenta son las siguientes:

- La pureza de $\mathrm{H}_{2}$ de ciertas corrientes de aporte fresco $\mathrm{y}_{\mathrm{LPH}}{ }^{\mathrm{H} 2}, \mathrm{y}_{\mathrm{H} 3}{ }^{\mathrm{H} 2}$;

- La disponibilidad de caudal de aporte fresco desde el colector de baja pureza $F_{L P H}$, ya que depende de otras plantas consumidoras de $\mathrm{H}_{2}$;

- El precio del $\mathrm{H}_{2}$ de alta pureza producido en las plantas productoras mediante reformado de metano con vapor.

\section{A.8.2.2- Análisis de resultados}

Para identificar las regiones de restricciones activas, se optimiza con respecto a los grados de libertad disponibles para un conjunto de distintos escenarios sometidos a distintas perturbaciones. Se analizan un total de 114 casos de estudio. Las regiones activas han sido identificadas y analizadas. A pesar del escaso número de grados de libertad y restricciones, el número de regiones activas resultante es elevado.

Para cada región, ciertas variables manipuladas están en alguno de sus límites del rango de operación, y el resto de variables manipuladas se pueden emparejar con una restricción activa. La pureza de $\mathrm{H}_{2}$ del reciclo $Y_{R E C}{ }^{H 2}$ es casi siempre restricción activa; aunque el método de Skogestad propone elegir variables con buena controlabilidad, $y_{R E C}{ }^{H 2}$ es la variable controlada más natural para la optimización económica, para ajustarse a la especificación mínima requerida en dicha variable. Su mala dinámica es debida al retardo de transporte que involucra al reactor, al separador de alta presión y la corriente de reciclo.

De acuerdo a los resultados obtenidos, sólo en dos regiones, ninguna de ellas frecuente, surge un compromiso entre dos variables manipuladas. Por tanto, sólo en dos regiones hay necesidad de buscar variables de control self-optimizing para hacer uso de los grados de libertad económicos restantes no usados o no en restricción. Como consecuencia, una operación óptima está asegurada en la mayor parte de los escenarios incluso en presencia de perturbaciones o incertidumbre, excepto por dos regiones con baja probabilidad de ocurrencia.

Como consecuencia del potencial cambio de operación entre regiones activas, la implementación de la estructura de control resultante, con emparejamientos entre variables controladas y manipuladas, puede ser trabajosa. Una opción sería programar la lógica de los emparejamientos en una capa superior. En lo que se refiere a algunas variables de decisión importantes, hay una estructura que siempre se repite en las distintas regiones activas: primero se controla con la entrada a membranas $F_{I N}$; cuando ésta se satura, se controla con la purga de membranas $F_{F G_{-}} ;$y cuando ésta también se satura, se controla con la purga directa a FG $F_{F G_{-} H P \text {. }}$

\section{A.8.2.3- Conclusiones}


La técnica de optimización por control self-optimizing es robusta ante la incertidumbre cuando se compara con el RTO (Skogestad, 2000). Otra gran ventaja es que es fiable incluso en presencia de errores de modelado; aunque la validación del modelo empleado no es perfecta cuantitativamente con errores de hasta el $10 \%$ en la predicción del consumo de hidrógeno, el análisis realizado en relación a las regiones activas es correcto.

La estructura de control resultante para la planta HDS es sencilla y fácil de implementar (control feedback con cierta lógica programada en una capa superior), y garantiza una operación óptima en la mayor parte de los escenarios, aunque se necesitaría una capa superior de RTO para asegurar una operación en la región correcta, con actualizaciones poco frecuentes. Los mayores inconvenientes son debidos al nivel de automatización de la planta, que ha de ser elevado para la implementación de esta tecnología; en particular la unidad de membranas no puede ser operada manualmente. Los retardos de transporte debidos a la dinámica introducida por el sistema reactor-separador HP-reciclo no son despreciables, haciendo que un enfoque de MPC con una función objetivo económica pudiera estar justificado, especialmente si se tienen en cuenta el elevado número de regiones de restricciones activas, dado su potencial para gestionar restricciones de manera sencilla.

El siguiente paso sería la aplicación de la técnica de optimización de self-optimizing a la optimización de la operación en tiempo real de la red global de la refinería. La política óptima self-optimizing aplicada a una planta consumidora aislada puede ser subóptima a nivel de la red global, por no explotar debidamente todas las interacciones. A escala de la red global, se aplicará un enfoque de RTO. No obstante, aunque la estructura de la red sea compleja, el número de combinaciones permitidas no es elevado, y se pueden buscar estructuras de control self-optimizing con el fin de simplificar el problema y reducir las pérdidas entre ejecuciones sucesivas del RTO.

\section{A.8.3- MODELO DE LA RED DE HIDRÓGENO}

El propósito del modelo, la optimización de la operación de la red global, ha determinado el alcance del modelo. Sólo se han formulado balances de materia sencillos, por las siguientes razones:

- Los balances de cantidad de movimiento introducen un nivel muy elevado de complejidad y detalle. Las restricciones relacionadas con flujos se han especificado a partir de valores históricos, en lugar de ser el resultado de relaciones caudal-presión.

- El nivel de complejidad se ha seleccionado teniendo en cuenta la información disponible online. De otro modo, se presentarían subconjuntos de variables no observables, dificultando la calibración del modelo debido a la complejidad de las mezclas procesadas y la variabilidad experimentada por el proceso. Por esta razón no se han incluido balances de energía. 
Sólo se han tenido en cuenta las unidades y fenómenos relacionados con el $\mathrm{H}_{2}$ : corrientes de gas, reactores, separadores, membranas, compresores, mezcladores, divisores y válvulas. Los balances de materia propuestos son simplificados, con el objetivo de facilitar la calibración en línea del modelo previa a la etapa de optimización. Los efectos dinámicos se han despreciado, es decir, los modelos son estacionarios para un periodo promediado. Se han elegido valores promedio de dos horas, mayores que el retardo de transporte en el sistema HP y por tanto englobando los efectos dinámicos en cierta medida.

\section{A.8.3.1- Modelo de corrientes de gas}

El modelo del sistema consiste en balances de materia a corrientes de gas, que se suponen constituidas por dos componentes: hidrógeno $\mathrm{H}_{2}$, y el resto de gases ligeros diferentes del hidrógeno englobados en un único componente llamado LIG. Aunque los componentes individuales que forman LIG no se consideran, sí se considera el cambio en su peso molecular $w^{L / G}$ debido a que: i) se necesita para la compensación en línea de las medidas de caudal disponibles, por el tipo de medidor de placa y orificio, siendo la compensación muy sensible a su valor dada la gran diferencia con respecto al $w^{\text {H2 }}$ de $2.016 \mathrm{~kg} / \mathrm{kmol}$; ii) existen diferencias significativas en $w^{L / G}$ según el tipo de corrientes, dependiendo especialmente de la presión; iii) en el sistema HP se puede estimar con más exactitud, con menor variabilidad de proceso.

Por tanto se necesitan tres balances para modelar las corrientes de gas. Una caracterización completa de una corriente de gas $i$ se realiza con las siguientes variables independientes:

- Caudal en condiciones Normales, equivalente a flujo molar: $\boldsymbol{F}_{i}\left(\mathrm{Nm}^{3} / \mathrm{h}\right)$

- Pureza de hidrógeno: $\quad y_{i}{ }^{H 2}(\% 1 \mathrm{~mol})$

- Peso molecular de gases ligeros: $\quad \boldsymbol{w}_{i}^{\text {LIG }}(\mathrm{kg} / \mathrm{kmol})$

\section{A.8.3.2- Modelo del Reactor}

El reactor es catalítico de lecho fijo, con flujo bifásico. Se ha modelado con los siguientes parámetros independientes para modelar los cambios experimentados por la fase gas a su paso:

- Consumo de $\mathrm{H}_{2}$ :

$$
\Delta \boldsymbol{R}_{H 2}\left(\mathrm{Nm}^{3} \mathrm{H}_{2} / \mathrm{h}\right)
$$

- Generación de ligeros en flujo molar: $\quad \Delta \boldsymbol{R}_{\mathrm{LIG}}\left(\mathrm{Nm}^{3} \mathrm{LIG} / \mathrm{h}\right)$

- Peso molecular promedio de los ligeros generados, equivalente a generación de ligeros en flujo másico:

$$
\Delta \boldsymbol{R}_{w L G}\left(\mathrm{~kg}^{\mathrm{LG}} / \mathrm{kmol}^{\mathrm{LG}}\right) \quad\left[\Delta \boldsymbol{R}_{w}(\mathrm{~kg} \mathrm{LIG} / \mathrm{h})\right]
$$

Las siguientes consideraciones permiten una mayor comprensión de los parámetros:

- Los consumos específicos de $\mathrm{H}_{2}\left(\Delta R_{\mathrm{H} 2}{ }^{5 p}\right)$ por hidrocarburo procesado se determinan en el problema de reconciliación de acuerdo con medidas en línea de caudal y analizadores de pureza. 
- Para cada planta consumidora, se han especificado rangos estrechos para los parámetros intensivos $\Delta R_{\text {wLIG }}$ y $\Delta R_{L I G H 2}$, siendo este último la relación de generación de ligeros a consumo de hidrógeno, de acuerdo con datos de diseño y conocimiento del proceso.

\section{A.8.3.3- Modelo del Separador}

Se ha considerado un modelo simplificado para las relaciones de solubilidad, debido a la falta de medidas en línea relativas a composición y la alta variabilidad del proceso. De acuerdo con la presión y temperatura de operación, y también la composición del hidrocarburo líquido, parte del gas permanece disuelto en el líquido a la salida mientras que el resto es separado en una corriente de gas de salida. El tiempo de residencia es suficiente para alcanzar el equilibrio.

Además de los tres balances de materia, se definen tres relaciones para el equilibrio de solubilidad, mediante los tres parámetros independientes siguientes:

- Solubilidad total de gas en el hidrocarburo líquido: $\boldsymbol{k}_{\text {gasHC }}^{s}\left(\mathrm{Nm}^{3} \mathrm{Gas} / \mathrm{m}^{3} \mathrm{HC}\right)$

- Coeficiente de distribución relativa de $\mathrm{H}_{2}$ y ligeros entre las fases gas y líquida:

$$
\boldsymbol{k}_{\text {aH2LIG () }}^{\text {s }}
$$

- Coeficiente de distribución relativa de ligeros según su peso molecular, entre las fases gas y líquida:

$$
\boldsymbol{k}_{\text {wLIG }}^{\text {s. }}\left(\mathrm{kg} \cdot \mathrm{kmol}^{-1} / \mathrm{kg} \cdot \mathrm{kmol}^{-1}\right)
$$

Las siguientes consideraciones permiten una mayor comprensión de los parámetros:

- El parámetro $k_{\text {gasHc }}^{S}$ implica que el hidrocarburo líquido se encuentra saturado en gas, lo que es consistente con la experiencia real en la operación.

- Debido a que la naturaleza de los gases ligeros no cambia significativamente, y las condiciones de operación de presión, temperatura y composición de hidrocarburo en los separadores varían en un rango limitado, los parámetros $k_{\alpha H 2 L G G}^{S}$ y $k_{w L I G}^{S}$ se pueden acotar en un rango estrecho. Es una hipótesis aceptable puesto que las impurezas o ligeros se generan en los mismos procesos, estando siempre presentes en cierta proporción $\mathrm{C}_{2} \mathrm{H}_{6}, \mathrm{C}_{3} \mathrm{H}_{8}$ and $\mathrm{C}_{4}$. Un cambio significativo sería unos ligeros con sólo $\mathrm{CH}_{4}$ como impureza, lo cual afectaría a la solubilidad relativa.

- Como la temperatura está controlada, los parámetros $k_{\alpha H 2 L I G}^{S}$ y $k_{w L I G}^{S}$ se han especificado en función de la presión e hidrocarburo procesados en cada caso, y sus valores para distintas plantas son coherentes.

El modelo tiene las siguientes características:

- Es sencillo y no riguroso, pero es físicamente consistente y tiene significado físico. Además puede ser calibrado en línea para la variable a la que es más sensible, el caudal de gas disuelto en el líquido del separador HP, que es separado aguas abajo en las separaciones MP/LP y medido.

- Es coherente con la evidencia experimental. 
- Es suficientemente flexible para ajustar el rango de composiciones deseado en todos los separadores, de acuerdo con datos históricos de composición de laboratorio. Simultáneamente, la sobreparametrización se ha evitado acotando en rangos estrechos los valores de los parámetros $k_{\alpha H 2 L I G}^{S}$ y $k_{w L I G}^{S}$.

- A diferencia de otros modelos de equilibrio de la literatura, no se basa en composiciones individuales de gas ni hidrocarburo, que no son medidas en línea.

\section{A.8.3.4- Implementación del modelo en el entorno EcosimPro ${ }^{\circledR}$}

El modelo de la red se ha implementado en el entorno orientado a objetos EcosimPro ${ }^{\circledR}$, que permite simular sistemas algebraico-diferenciales con eventos discretos.

El modelado se organiza jerárquicamente de modo gráfico. Los componentes individuales para modelar la red se han desarrollado en la librería gráfica H2NET.

Como rasgo distintivo, se incluyen dos puertos para modelar corrientes de gas: el puerto " $h 2$ ", para corrientes con un sentido único de flujo, y el puerto "h2dir", que permite modelar corrientes cuyo sentido de flujo no se conoce a priori pudiendo ser ambos el directo y el inverso. En la red, esta situación tiene lugar en colectores y tuberías de unión entre plantas. El modelado de componentes mezcladores o divisores con corrientes caracterizadas con puertos " $h 2 d i r$ " es diferente en el sentido de que ambas situaciones de flujo directo o inverso han de tenerse en cuenta en el modelo, porque el sentido de flujo no depende del componente individual sino del balance de flujos a nivel de la red. Por cuestiones de simetría y causalidad física, es necesario incluir una variable auxiliar para modelar un igual número de ecuaciones con independencia del sentido de flujo.

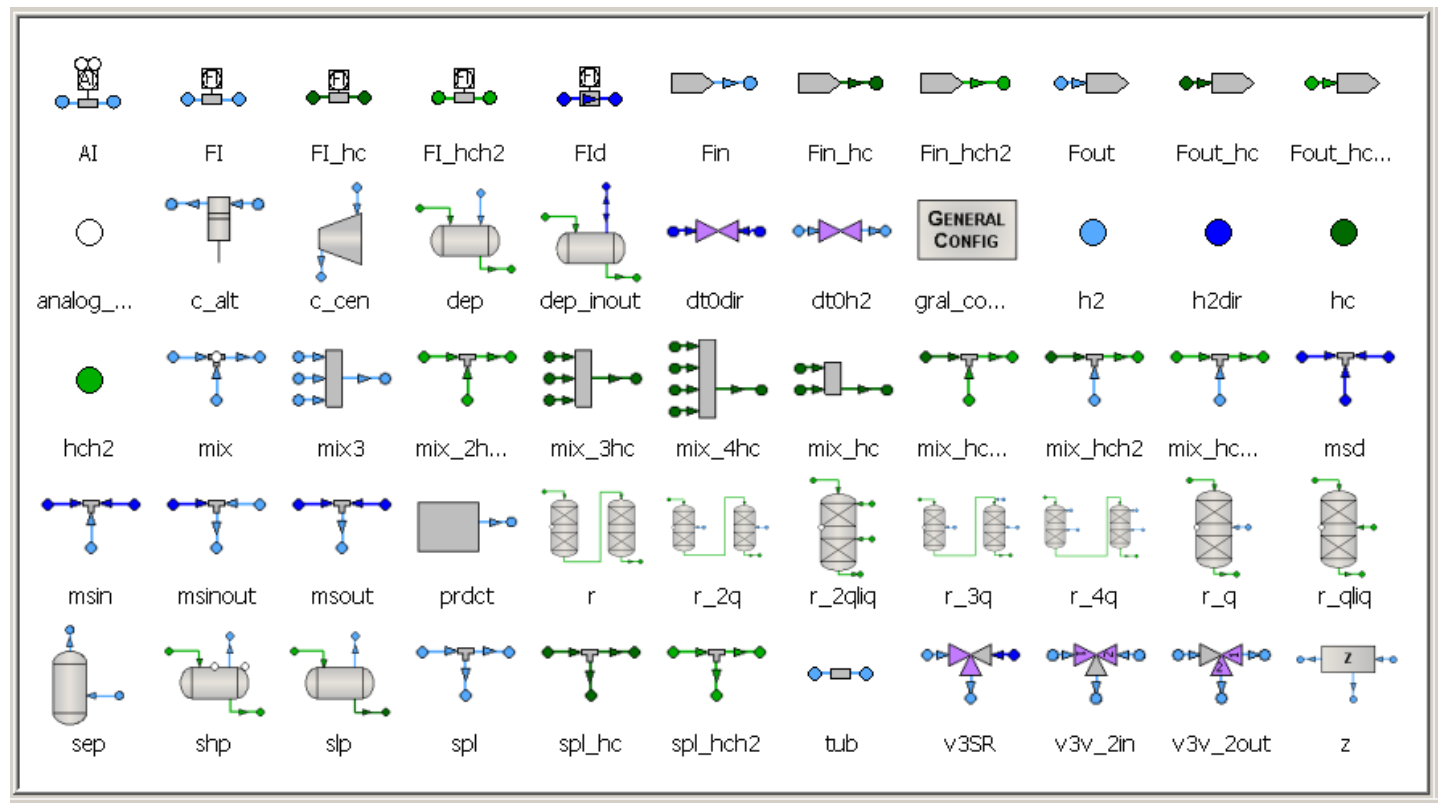

Figura A.7. Componentes de la librería gráfica H2NET desarrollada en EcosimPro ${ }^{\circledR}$. 
En la memoria se incluyen el detalle y consideraciones necesarios para:

- Seleccionar las entradas o boundaries a especificar en la simulación, de acuerdo con la causalidad física del sistema, siguiendo un orden de aguas arriba a aguas abajo.

- Características adicionales de la librería, útiles para la generación automática del código para implementar los dos problemas de optimización para resolver la reconciliación de datos y la redistribución óptima. Se generan así vectores de variables de decisión, restricciones, sumandos en la función de coste, límites para las variables de decisión y las restricciones, información sobre si un flujo es medido y el tag correspondiente, información sobre si existe válvula en una línea y el tag de la apertura correspondientes.

- $\quad$ Adquisición de datos. Se incluye un esquema con la estructura para configurar el modelo, leer datos reales de proceso, y ejecutar un experimento de simulación.

- Tratamiento de datos. Previo a su uso en la simulación, los datos de proceso son procesados para eliminar posibles errores de lectura o medida. Se lleva a cabo de acuerdo con el tipo de variable, y los valores del dato bruto, la desviación estándar de la medida, y en algunos casos la apertura de la válvula correspondiente. En el caso de que se detecte una medida no fiable, se le asigna un peso nulo en el problema de reconciliación de medidas.

\section{A.8.3.5- Conclusiones}

- Es un modelo de primeros principios, con buenas capacidades de predicción.

- Los parámetros propuestos tienen significado físico, lo que ayuda en el conocimiento del proceso y en la comparación de valores entre unidades similares.

- El modelo es lo suficientemente sencillo como para ser calibrado y actualizado en línea.

- El modelo de corrientes de gas con dos sentidos de flujo permitidos proporciona cierta flexibilidad en la optimización en lo que respecta a decisiones topológicas, siendo a la vez un enfoque sencillo comparado con un modelado híbrido usando variables de decisión binarias.

- No se ha realizado un estudio sistemático de sensibilidad e identificabilidad de parámetros. La identificabilidad se ha resuelto con rangos estrechos para ciertos parámetros de acuerdo con el proceso y las condiciones de operación, reduciendo así los efectos de la sobreparametrización. 
- Con el propósito de permitir una mayor flexibilidad y variabilidad en algunos parámetros del modelo, sin comprometer la robustez y la consistencia, se podrían estudiar correlaciones en función de variables medidas para el peso molecular de los ligeros generados en las unidades de platformado, o los coeficientes de solubilidad relativa en función de la temperatura y la composición en los separadores HP de algunas plantas importantes.

\section{A.8.4- RECONCILIACIÓN DE DATOS DE LA RED DE HIDRÓGENO}

\section{A.8.4.1- Motivación}

La reconciliación de datos en la red de hidrógeno es un problema interesante debido a la dificultad en la estimación exacta del estado de la planta. La incertidumbre es debida a:

- Una gran variabilidad en las condiciones de operación y en el consumo de hidrógeno.

- La escasez de medidas en línea para la pureza de $\mathrm{H}_{2}$ y el peso molecular del gas. Los elevados costes de instalación y mantenimiento de los analizadores hacen que no siempre estén justificados en la industria de procesos.

- El uso de caudalímetros de tipo placa y orificio para la medida de caudal de las corrientes de gas a pesar de ser propensos a error, debido a que es la tecnología más barata disponible en el mercado. Los potenciales errores de deriva están causados por: i) variabilidad del proceso, con condiciones diferentes de $[P, T, W]$ en la operación respecto de las correspondientes a diseño/calibración; ii) el transmisor de presión diferencial, cuando el flujo medido se encuentra en las zonas inferior y superior del rango de medida; iii) inexactitudes en las compensaciones, por ejemplo caudalímetros que no disponen de medidor de presión y es necesario utilizar alguno próximo aguas arriba o aguas abajo de una válvula, o caudalímetros sin medida de temperatura disponible en la tubería.

- El hecho de que el peso molecular del gas $w$ experimente variaciones significativas para pequeños cambios en la composición de los principales ligeros presentes, $\mathrm{CH}_{4}, \mathrm{C}_{2} \mathrm{H}_{6}$, and $\mathrm{C}_{3} \mathrm{H}_{8}$, debido al bajo valor del peso molecular del $\mathrm{H}_{2}$ comparado con el de las principales impurezas, cuyos $w$ son $2,16,30$ y 44 respectivamente. Esta es una diferencia importante respecto de otras redes de gas como las redes de gas natural, cuya composición es constante.

- En algunos casos, una instalación o mantenimientos no adecuados pueden ser responsables de errores sistemáticos en la medida. 


\section{A.8.4.2- Enfoque y Formulación del problema de Reconciliación de Datos}

El enfoque para abordar la incertidumbre ha sido el siguiente:

- Se han descartado los instrumentos con errores sistemáticos grandes, que normalmente son persistentes en el tiempo.

- Se ha utilizado el modelo simplificado de solubilidad presentado para determinar el factor (purgas de MP/LP) que menos contribuye a las necesidades de $\mathrm{H}_{2}$, siendo a la vez capaz de proporcionar estimaciones consistentes.

- Se ha ejecutado una reconciliación de datos en línea mediante técnicas de optimización para estimar el consumo de $\mathrm{H}_{2}$ en los reactores y el estado de las plantas de acuerdo con los caudalímetros (FI) y analizadores (AI) disponibles, con el fin de obtener una estimación consistente del estado de la red.

El problema se formula como la solución de la minimización de la suma de los cuadrados del error para las desviaciones medida compensada-modelo, sujeto a las restricciones de igualdad del modelo y a otras restricciones de desigualdad como los límites en ciertas variables.

La referencia para los caudales del modelo no es la medida bruta, sino la medida compensada, donde el factor de compensación $\beta_{i}^{\text {comp }}$ permite compensar las desviaciones en las condiciones de operación respecto de las condiciones fijas de diseño/calibración de la placa de orificio, en presión, temperatura y peso molecular de la corriente de gas. El factor de compensación se deriva de los balances de materia y energía aplicados al cálculo del caudal que circula por la placa medido a partir de un transmisor de presión diferencial. El factor de compensación $\beta_{i}^{\text {comp }}$ se ha truncado arbitrariamente al rango [0.80, 1.20], de acuerdo con la experiencia práctica acerca del rango válido para la corrección: la medida compensada no es muy fiable para valores demasiado alejados de la unidad.

Las desviaciones de medida de los analizadores se han gestionado como restricciones no lineales en lugar de incluirse en la función de coste a minimizar, debido a la dificultad numérica de balancear términos no homogéneos en la función de coste.

Algunos parámetros de sintonía son muy importantes para conseguir unos resultados robustos y consistentes evitando los óptimos locales. En concreto el factor que permite computar los límites inferior y superior permitidos para cada caudal medido, como un rango centrado en la medida bruta ha resultado determinante, forzando acotar convenientemente la región de búsqueda. Un valor distinto se ha configurado para cada caudalímetro individual, lo menor posible según el factor de compensación esperado, los desbalances esperados en nodos redundantes para evitar no factibilidades, y la variabilidad esperada en el peso molecular de la corriente. Asimismo la tolerancia permitida en las medidas de pureza de los analizadores también ha condicionado significativamente el ajuste, dado que son variables a las que el sistema muestra una gran sensibilidad. 


\section{A.8.4.3- Validación y Análisis de resultados}

La validación conjunta del modelo y la reconciliación de datos se ha efectuado calculando tendencias fuera de línea, cubriendo periodos amplios de operación de varios días en diferentes escenarios. También se han seleccionado periodos correspondientes a cambios en las condiciones de operación para evaluar los resultados antes, durante y después del estado transitorio. La validación, y por tanto una estimación fiable del estado de la red, se da por correcta de acuerdo con los siguientes resultados:

- Aunque el modelo está sobreparametrizado, las tendencias en los datos reconciliados son consistentes en el tiempo. Los efectos de la sobreparametrización se han compensado con rangos acotados estrechos para ciertos parámetros de acuerdo con el conocimiento del proceso, sin perder flexibilidad en el ajuste.

- Los multiplicadores de Lagrange activos para las restricciones de caudal en las soluciones reconciliadas se corresponden en general con caudales ligados al sistema de alta presión HP, no a los de MP/LP donde hay mayor escasez de medidas.

- El chequeo de la consistencia en válvulas importantes de alivio que sólo abren ocasionalmente en ciertos transitorios y donde no se dispone de medida de caudal se ha efectuado de acuerdo con la apertura de la válvula. Cuando dicha válvula abre, se pierde una redundancia; es un buen signo que los mismos resultados se obtienen antes, durante y después del transitorio, con independencia de la apertura de la válvula.

- La consistencia y robustez en el ajuste para todas las corrientes importantes se ha efectuado por comparación con las tendencias en las medidas brutas, en particular para todas las corrientes de aporte desde colectores a las plantas consumidoras y de purgas de HP. Puede verse que los errores son sistemáticos, y que las tendencias monótonas crecientes o decrecientes se reproducen en los valores reconciliados. Desviaciones similares en signo y magnitud se obtienen para distintos periodos de operación.

- Cuando un instrumento no dispone de redundancia ni local ni global, el ajuste del modelo debería ser perfecto, como es el caso. En algunos colectores importantes sin embargo, el desbalance cuando sólo se consideran medidas compensadas es menor que la suma de los valores absolutos de los residuos modelo-medida compensada para todos los caudalímetros vinculados al mismo. Es decir, el desbalance inicial se distribuye dando lugar a mayores desviaciones de lo esperado, de modo que se podría haber obtenido una solución con menores desviaciones para dicho colector aislado, pero no es el caso debido al ajuste global de la red con todas las redundancias y restricciones. Si el funcionamiento del resolvedor matemático es el adecuado, y la experiencia lo confirma, este hecho se 
puede interpretar como una evidencia adicional de la existencia de errores sistemáticos en los instrumentos.

A continuación se muestran resultados para algunas variables importantes. En cada figura se comparan tres valores, el dato bruto (dtF), la medida de caudal compensada $(F v c)$ con presión, temperatura y peso molecular en la operación, donde el peso molecular se obtiene a partir del modelo, y el valor del modelo (Fmdl) solución del problema de reconciliación.

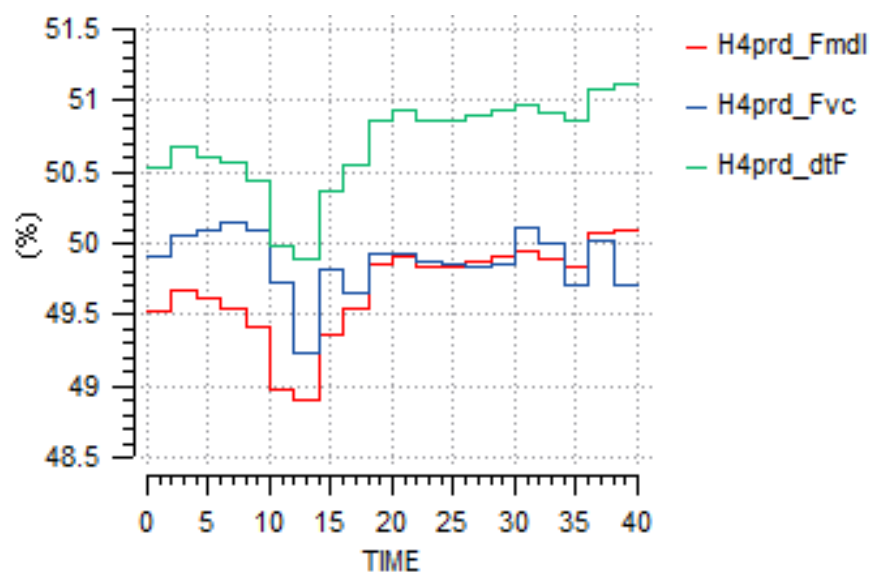

Figura A.8. Producción de $\mathrm{H}_{2}$ en una planta con horno de reformado de gas natural.

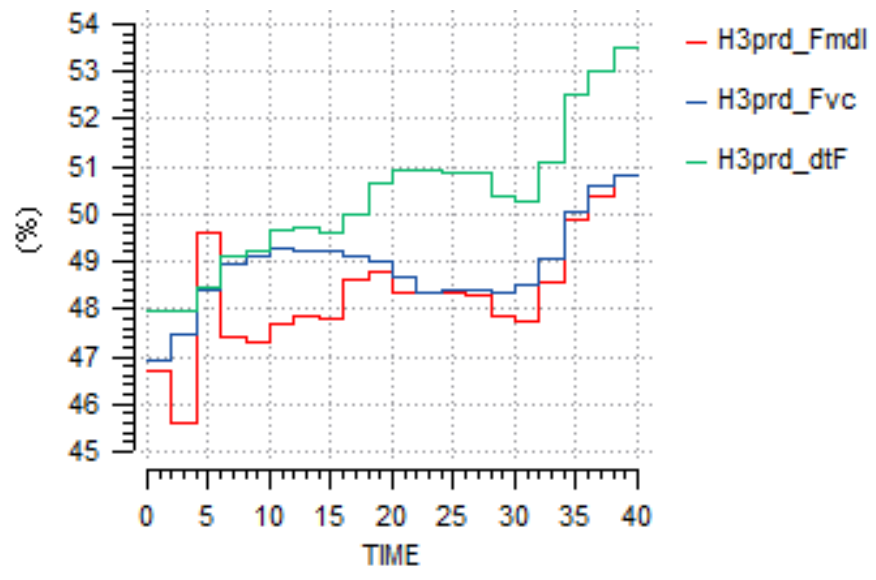

Figura A.9. Producción de $\mathrm{H}_{2}$ en una planta con horno de reformado de gas natural.

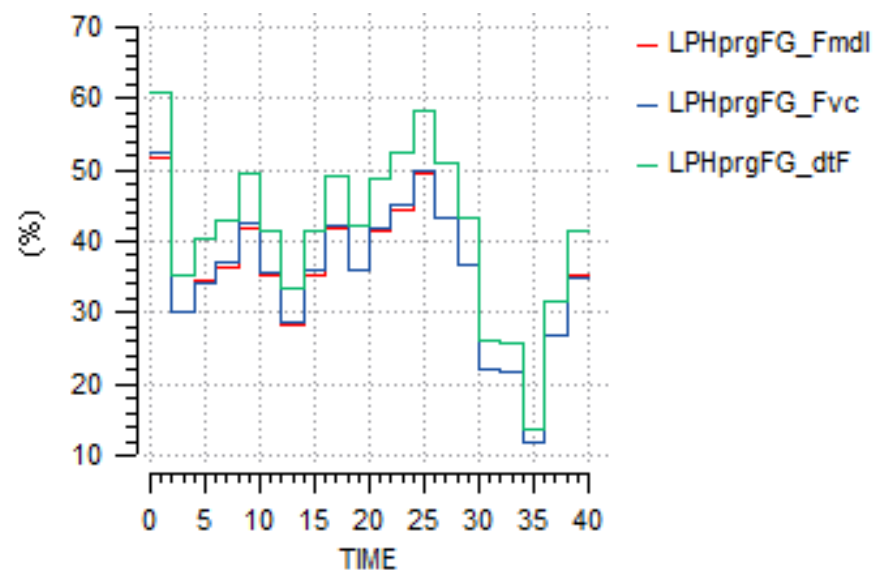

Figura A.10. Purga a fuel gas FG desde el colector de baja pureza LPH. 

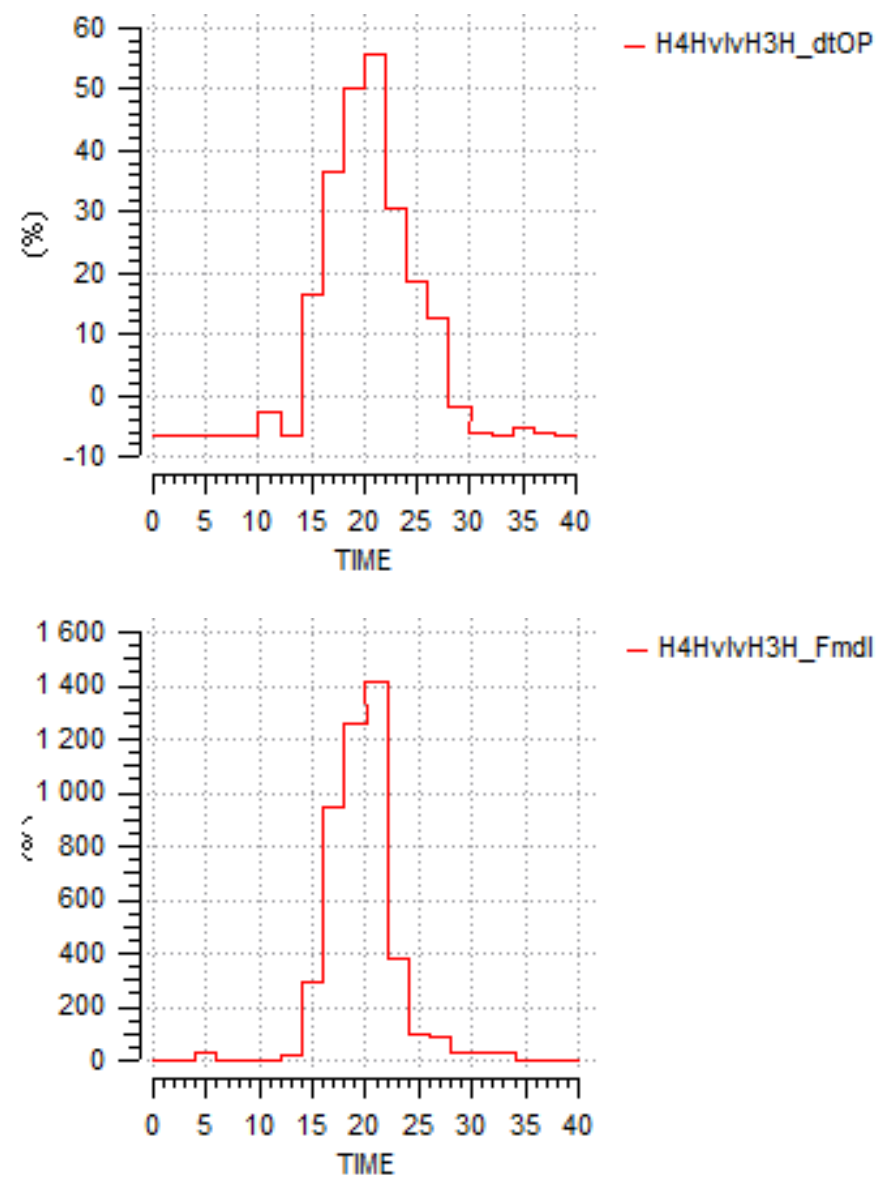

Figura A.11. Apertura de válvula y caudal estimado para el alivio desde el colector de $\mathrm{H} 4$ al colector de $\mathrm{H} 3$.

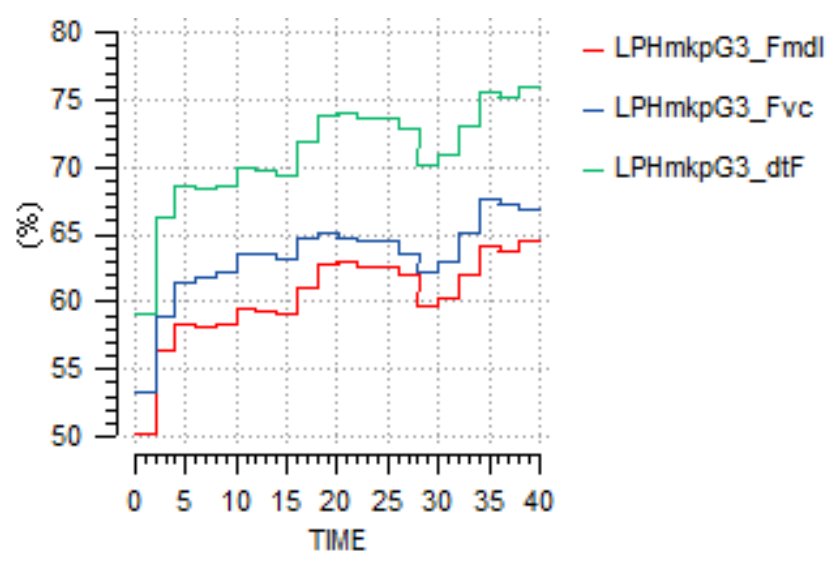



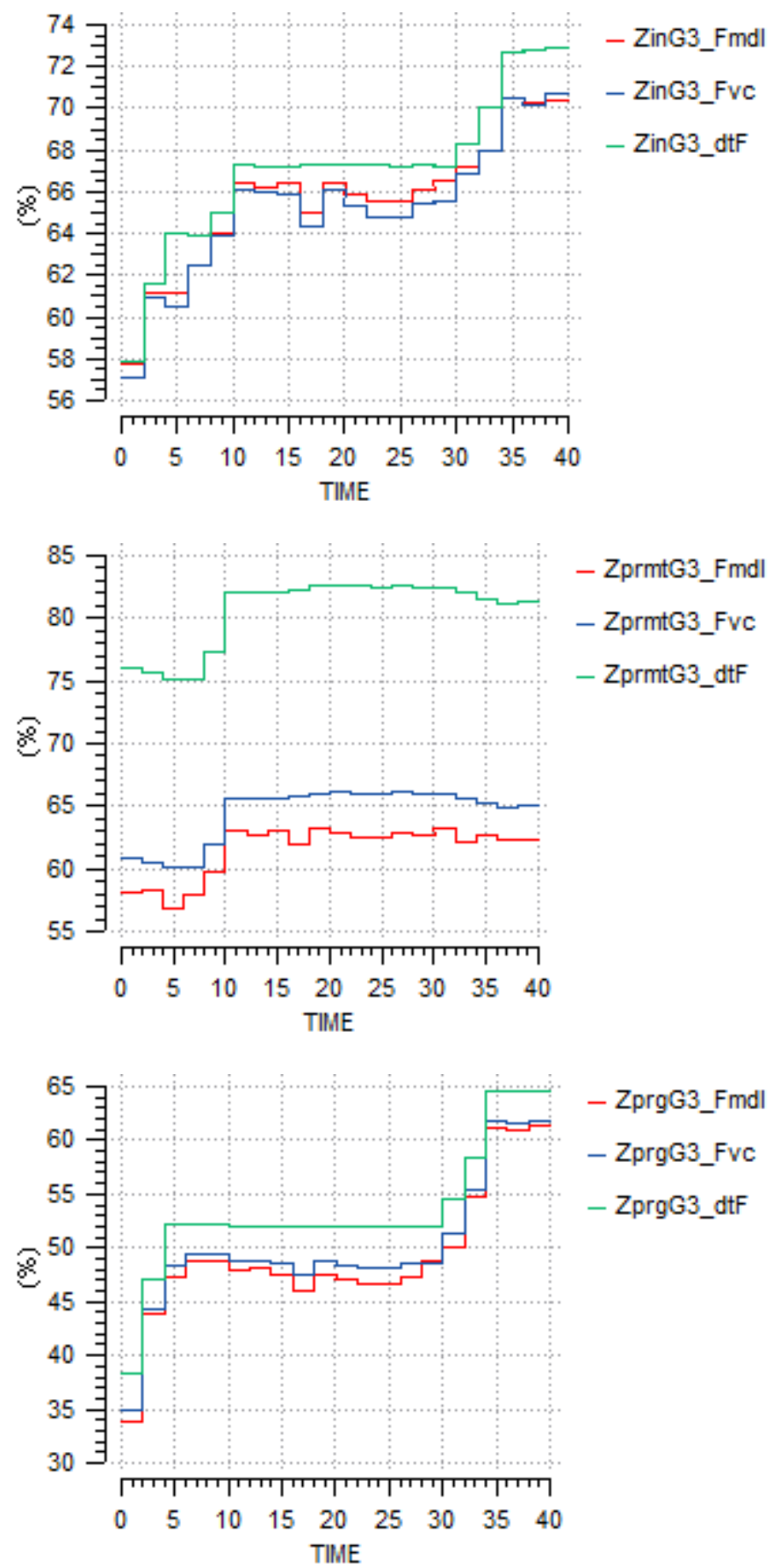

Figura A.12. Caudal de aporte desde el LPH, y caudales de entrada, permeado y purga en las membranas de una planta consumidora importante.

\section{A.8.4.4- Conclusiones}

- El modelo subyacente ha permitido detector y descartar instrumentos afectados por errores sistemáticos importantes, que son persistentes.

- La secuencia seguida ha permitido una comprensión adecuada del problema. Primero se calibraron manualmente en simulación los modelos para todas las unidades, usando como referencia datos de diseño y laboratorio. Asimismo se 
estudiaron las sensibilidades y compromisos en el ajuste. Finalmente, se sintonizaron con cierta precisión aquellos parámetros y límites más importantes para el ajuste global.

- Los resultados son satisfactorios, garantizando la consistencia y robustez a expensas de una cierta reducida flexibilidad según lo esperado por la variabilidad en el proceso en ciertos parámetros del modelo, aunque sin comprometer la exactitud de los resultados. Esto es así para evitar los efectos indeseados de los óptimos locales, cierto grado de sobreparametrización y poca identificabilidad, y la incertidumbre en las medidas disponibles.

\section{A.8.5- REDISTRIBUCIÓN ÓPTIMA DE LA RED DE HIDRÓGENO}

La redistribución óptima de $\mathrm{H}_{2}$ se resuelve como la solución matemática del problema no lineal de minimización del coste de operación, sujeto a las restricciones del modelo y del proceso, de acuerdo con un enfoque de optimización RTO. Se ha seleccionado la técnica RTO por la complejidad y la naturaleza multivariable de todas las interacciones entre plantas a nivel de la red. El estado inicial de la planta es el obtenido como solución del problema de reconciliación de medidas previo.

\section{A.8.5.1- Formulación de la Operación Óptima}

Sólo se consideran los costes de producción de $\mathrm{H}_{2}$, pero no el valor como fuel gas de las corrientes de purga. Como el margen en precios es alto, apenas influirá en los resultados; por otra parte, se desea una producción tan baja como sea posible, y este término competiría con ese objetivo. Por la misma razón, también se considera nulo el precio del $\mathrm{H}_{2}$ de baja pureza de los platformados. Como los costes materiales del $\mathrm{H}_{2}$ son aproximadamente un orden de magnitud superiores de los costes de la energía de compresión, estos últimos no se han tenido en cuenta.

Las hipótesis consideradas en la resolución son las siguientes:

- No hay $\mathrm{H}_{2}$ ni gases ligeros disueltos en las entradas y salidas de hidrocarburo de acuerdo con las condiciones de proceso;

- El modelo simplificado de solubilidad se usa para calcular el $\mathrm{H}_{2}$ y los gases disueltos en el hidrocarburo líquido de salida de los separadores de HP/MP, con iguales valores de los parámetros $\left(k_{\text {gasHC, }}^{S} k_{\alpha H 2 L G}^{S}, k_{w L I G}^{S}\right)$ que aquellos estimados en la etapa de reconciliación. Es equivalente a considerar que la corriente de hidrocarburo está saturada en gases disueltos, suponiendo una operación constante referida a: i) temperatura $\mathrm{T}_{\mathrm{HP} / \mathrm{MPsep}}$, controlada en el mínimo; ii) presión $\mathrm{P}_{\text {HP/MPsep, }}$ regulada en un valor fijo; iii) composición y caudal de hidrocarburo. Por otra parte, la pureza en la salida de HP se controla en un valor mínimo 
especificado, por lo que las condiciones de operación en cuanto a composición del gas se mantienen en un rango estrecho.

- Los términos de generación y consumo en el reactor se admiten invariables: $\Delta R_{H 2}{ }^{s p}\left(\mathrm{Nm}^{3} \mathrm{H}_{2} / \mathrm{m}^{3} \mathrm{HC}\right), \Delta R_{L I G}{ }^{s p}\left(\mathrm{Nm}^{3} \mathrm{LIG} / \mathrm{m}^{3} \mathrm{HC}\right), \Delta R_{\text {wLIG }}$ ( $\mathrm{kg} \mathrm{LIG} / \mathrm{kmol} \mathrm{LIG)}$ ). Es equivalente a considerar una operación constante relativa a: i) calidad del hidrocarburo procesado; ii) cantidad del hidrocarburo procesado o tiempo de residencia en el reactor; iii) severidad en el reactor, es decir temperatura; iv) cinéticas de pseudo-orden cero respecto del $\mathrm{H}_{2}$, con velocidades de reacción independientes de su concentración y por tanto de una potencial redistribución de caudales.

- La operación de la red se mantiene en lo que respecta a decisiones topológicas, es decir, no se tienen en cuenta variables binarias en la optimización. En colectores o conexiones compartidas con dos sentidos permitidos para el flujo, ambos casos están contemplados en la resolución sin necesidad de incorporar variables binarias de acuerdo con el modelo y el puerto " $h 2 d i r$ ".

- Ciertos caudales se mantienen invariables en el valor estimado en la reconciliación, debido a que no influyen en la eficacia en el uso de $\mathrm{H}_{2}$ y su valor se fija de acuerdo con otros criterios de operación independientes del $\mathrm{H}_{2}$ : i) caudales de quench entre lechos del reactor para el control de temperatura; ii) corrientes de bypass; iii) caudales de reciclo; iv) corrientes separadas en elementos divisores aguas abajo de las operaciones de separación de MP/LP. Son caudales no manipulados libremente y separados bajo regulación de presión, por lo que un splitter aguas abajo no introduce grados adicionales de libertad. Corresponden a válvulas cerradas normalmente y que se activan como segunda rama en el control de rango partido para el alivio de presión; v) composición del gas en las plantas productoras: $y_{i}^{H 2}, w_{i}^{L / G}$.

Las restricciones de operación a cumplir son las siguientes:

- Pureza de $\mathrm{H}_{2}$ en el reciclo o sistema de HP, por razones de mantenimiento del catalizador evitando la deposición de carbono, y de asegurar el grado deseado de desulfuración. Es muy determinante.

- La proporción $\mathrm{H}_{2} /$ hidrocarburo a la entrada del reactor. También con el objetivo de asegurar exceso suficiente de $\mathrm{H}_{2}$ en el reactor.

- La capacidad de producción de las plantas productoras mediante horno de reformado de gas natural con vapor.

- La capacidad de las membranas y el rango de operación según caudal purgado respecto de la entrada.

- La capacidad de los compresores alternativos de aporte fresco, que pueden ser factor limitante en ocasiones. 
- La capacidad de ciertas tuberías, de acuerdo con datos históricos. Especialmente en las purgas de HP.

- El caudal mínimo a mantener como purga en ciertos colectores, en particular el $\mathrm{LPH}$, por motivos de controlabilidad y rechazo de perturbaciones.

\section{A.8.5.2- Implementación en EcosimPro ${ }^{\circledast}$}

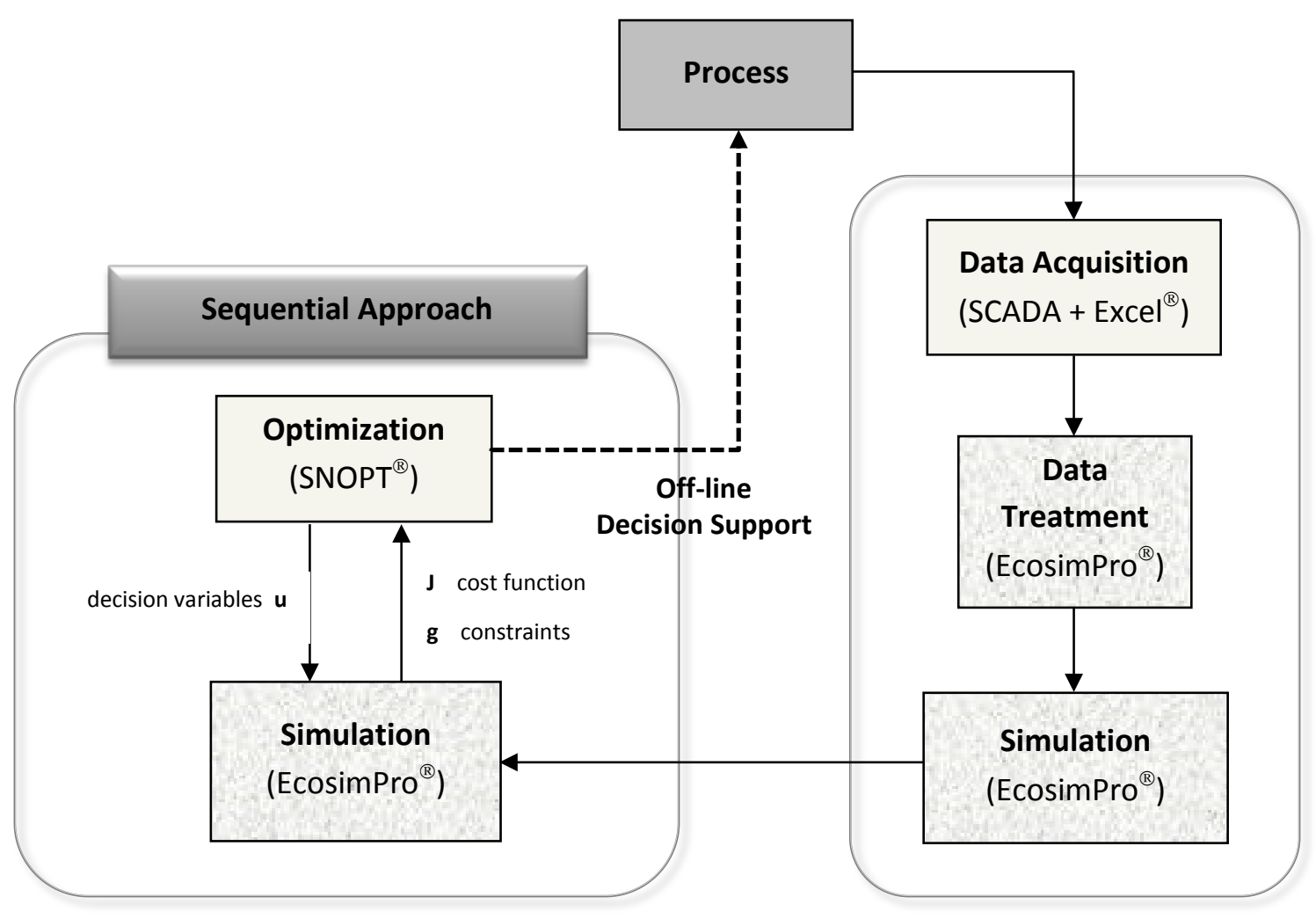

Figura A.13. Esquema de la implementación del problema de redistribución óptima de $\mathrm{H}_{2}$ en EcosimPro ${ }^{\circledR}$.

La implementación se ha efectuado en EcosimPro ${ }^{\circledR}$, con un algoritmo SQP como resolvedor, $\mathrm{SNOPT}^{\circledR}$, siguiendo un enfoque secuencial.

\section{A.8.5.3- Análisis de las Soluciones de Operación Óptima}

En el análisis efectuado para distintos escenarios cubriendo un rango amplio de condiciones de operación, todas las soluciones se corresponden con el patrón lógico explicado a continuación. En primer lugar se describe el marco para lograr una gestión eficiente en el uso de $\mathrm{H}_{2}$, y en segundo lugar se analizan las soluciones y la estrategia óptima. 
En la red global, se distinguen dos escenarios extremos según la disponibilidad de $\mathrm{H}_{2}$ en el colector de baja pureza LPH y en general según la disponibilidad de $\mathrm{H}_{2}$ de baja pureza, bien de platformados o de exceso en las unidades consumidoras y que puede ser aprovechado como aporte fresco en otras plantas consumidoras. Estos casos extremos son déficit y exceso de $\mathrm{H}_{2}$. Exceso cuando el $\mathrm{H}_{2}$ de baja pureza ha de ser purgado porque no es útil para satisfacer las restricciones de mínima pureza ${ }_{H_{P P}}{ }^{H 2}$; y déficit cuando todo el $\mathrm{H}_{2}$ de baja pureza disponible puede ser reutilizado, sustituyendo así al $\mathrm{H}_{2}$ de alta pureza de las plantas productoras mediante horno de reformado a partir de gas natural. En la práctica, los escenarios se encuentran entre ambos extremos, y comparten características. Un resumen del estado de la planta se muestra en la tabla A.1. El escenario de déficit o exceso depende del $\mathrm{H}_{2}$ generado como subproducto en las unidades de platformado, y asimismo del conjunto de especificaciones de purezas mínimas en $\mathrm{HP} \mathrm{y}_{H P}{ }^{H 2}$ a verificar.

\begin{tabular}{lll}
\hline ESCENARIO: DISPONIBILIDAD DE $\mathrm{H}_{2}$ EN LPH & EXCESO & DÉFICIT \\
\hline
\end{tabular}

- $\quad$ Altas/Bajas] especificaciones para la mínima

$\boldsymbol{y}_{\text {HPsep }}{ }^{\mathrm{H} 2}$ pureza de $\mathrm{H}_{2}$ en $\mathrm{HP}_{\text {SEP }}$ : [no todo/todo] $\mathrm{H}_{2}$ Altas, no todo Bajas, todo del LPH útil

- Valor mínimo $\boldsymbol{y}_{\text {HPsep }}{ }^{\boldsymbol{H} 2}$ es restricción activa en todas las plantas consumidoras: [suficiente/no Sí, suficiente suficiente] $\mathrm{H}_{2}$ del LPH

- Membranas en marcha [sí/no]: purgas [necesarias/no necesarias]

- Las purgas obligatorias a FG en $\mathrm{HP}_{\mathrm{SEP}}$ [no minimizadas/minimizadas]: aporte fresco desde [LPH $\circ \mathrm{H}_{2}$ de baja pureza/ $\mathrm{H}_{2}$ de productoras o de alta pureza]

\author{
Existe un gap con \\ respecto al \\ mínimo en ciertas \\ plantas: las \\ necesidades se \\ satisfacen \\ parcialmente con \\ $\mathrm{H}_{2}$ de alta pureza \\ de las productoras
}

Sí, necesarias No, no necesarias

No minimizadas, Minimizadas, $\mathrm{H}_{2}$ $\mathrm{H}_{2}$ de baja de alta pureza pureza

Tabla A.1. Estado y características de la red para los dos casos extremos relativos a la disponibilidad de $\mathrm{H}_{2}$ de baja pureza.

Hay tres categorías en cuanto a plantas consumidoras según la variable purga de alta presión $\mathrm{HP}$, ordenadas de acuerdo con un orden decreciente en cuanto a eficacia en el uso de $\mathrm{H}_{2}$ : i) purga HP al colector de baja pureza LPH. La pureza de $\mathrm{H}_{2}$ de la purga es la misma que la del sistema de HP ${Y_{H P S e p}}^{H 2}$ en todas las consumidoras; ii) purga HP al colector de FG a través de una unidad de membranas, por tanto recuperando parte del hidrógeno a la entrada en la corriente de permeado. En este caso, la pureza de $\mathrm{H}_{2}$ de la purga es menor que la ${ }_{\mathrm{HPSep}^{2}}{ }^{\mathrm{H}}$; iii) purga HP 
directamente al colector de fuel gas FG. De nuevo la pureza de $\mathrm{H}_{2}$ de la purga es igual a la de

$y_{\text {HPsep }}{ }^{\text {H2 }}$. Por tanto, este caso únicamente se da en plantas con una especificación baja de pureza para $y_{\text {HPsep }}{ }^{\mathrm{H}}$, no siendo posible la reutilización de los excesos de $\mathrm{H}_{2}$.

Los escenarios estudiados se corresponden con situaciones entre los dos casos extremos. El análisis de soluciones se realiza según el tipo de variable de decisión para la operación óptima de la red. Según su influencia y efecto sobre la eficacia en la gestión de $\mathrm{H}_{2}$, se agrupan en purgas de HP tanto de consumidoras como de colectores, y aportes frescos desde colectores a las plantas consumidoras.

\section{i) Purgas de alta presión HP}

La solución es la lógica, es decir purgar el exceso de $\mathrm{H}_{2}$ de baja pureza, de haberlo, a nivel de la red a través de la purga de HP a FG a menor pureza de $\mathrm{H}_{2}$ hasta que se sature dicha purga, siguiendo un orden creciente en cuanto a pureza de $\mathrm{H}_{2}$ para purgar a FG desde HP en las plantas consumidoras y colectores. El caudal de purga es máximo cuando se verifica la restricción de pureza mínima $y_{\text {HPsep }}{ }^{H 2}$. Un caudal de purga mayor implica un mayor aporte de hidrógeno de baja pureza, garantizando que la restricción $y_{\text {HPsep }}{ }^{\text {H2 }}$ sigue siendo activa; por tanto un mayor aporte fresco de baja pureza supone una reducción/sustitución en el aporte fresco de alta pureza de las productoras, con el consiguiente ahorro. El caudal de purga se aumenta para seguir verificando la restricción a pesar de la mayor entrada de gases ligeros con el aporte de baja pureza. En consecuencia, las purgas directas a FG en las plantas consumidoras están en uno de los tres estados: $\left[\boldsymbol{F}_{F G}=0 / \boldsymbol{F}_{F G}\right.$ aumenta progresivamente $/ \boldsymbol{F}_{F G}$ saturado cuando se alcanza el máximo aporte fresco de baja pureza, es decir, aumentos mayores son bien no factibles de acuerdo con la restricción $y_{\text {HPsep }}{ }^{H 2}$ o bien no eficaces conduciendo a un gap en dicha restricción que deja de ser activa].

En este sentido, la operación óptima se puede averiguar sin necesidad de un resolvedor matemático, aunque necesitando tiempos mayores para alcanzar operación estacionaria después de implementar una secuencia de cambios. Por tanto la herramienta puede ayudar en la rápida identificación de operaciones subóptimas y en la implementación de las políticas óptimas. 


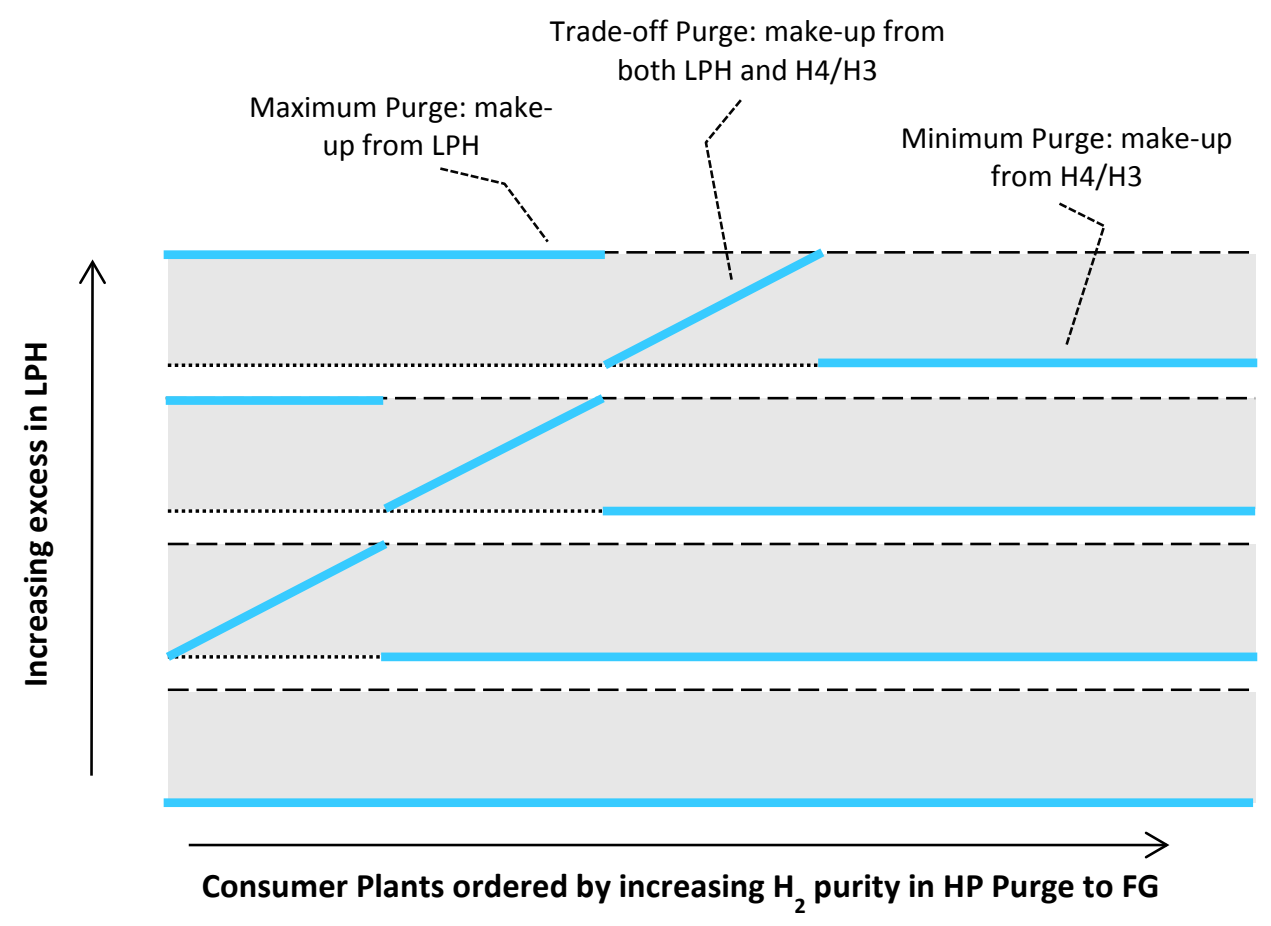

Figura A.14. Diagrama de política optima a nivel de la red global en relación a las purgas de alta presión HP a FG.

\section{ii) Aportes frescos desde las distintas plantas productoras}

En lo que se refiere a los potenciales compromisos en la redistribución del $\mathrm{H}_{2}$ desde las distintas plantas productoras (de horno de reformado a partir de gas natural) de alta pureza a las distintas consumidoras, el margen es apenas apreciable. La diferencia tanto en coste de producción como en pureza de $\mathrm{H}_{2}$ es muy pequeña, habiendo sin embargo un gap grande con respecto a la pureza en el LPH. Es decir, no es importante un aporte preferencial desde una u otra planta productora, pero sí aprovechar al máximo el hidrógeno de baja pureza disponible en el LPH.

Si se asegura una distribución uniforme o proporcionada del $\mathrm{H}_{2}$ del LPH entre las consumidoras más importantes (que suelen ser las que tienen mayor flexibilidad en cuanto a aportes desde distintos colectores) de acuerdo con las respectivas restricciones de $y_{H P_{s e p}}{ }^{H 2}$, entonces el margen en aportes preferenciales desde estas plantas productoras a las consumidoras, 0 intercambios entre aportes de productoras, es prácticamente despreciable para rangos amplios de caudales. Sin embargo el margen sí es significativo fuera de estos márgenes. La razón es evitar un gap subóptimo en las restricciones $\mathrm{y}_{\text {HPsep }}{ }^{\mathrm{H2}}$, y saturar el hidrocarburo líquido de salida del HP con un gas a la menor pureza posible.

\section{A.8.5.4- Conclusiones}

En lo que respecta al uso de la herramienta para la gestión en tiempo real de la red: 
- Hay oportunidad de ganancia aunque el margen no es elevado, debido a cambios frecuentes en los escenarios y las condiciones de operación y las grandes cantidades involucradas. Tampoco se pueden esperar grandes mejoras, puesto que las variables manipuladas en la redistribución (purgas HP) suponen alrededor del 3-5\% del total de la producción de $\mathrm{H}_{2}$.

- Las variables más importantes que monitorizar de acuerdo con la identificación de políticas subóptimas, y sujetas a cambios son: aportes frescos desde el LPH a las consumidoras importantes; purgas directas de HP a FG para algunas consumidoras de tamaño medio; purgas a FG desde la unidad de membranas, así como el caudal de aporte a dichas membranas.

- A pesar de la complejidad de la red y de las potenciales conexiones, los grados de libertad disponibles están reducidos debido a caminos preferenciales y razones operativas. La hipótesis de no considerar variables binarias está en concordancia con los resultados obtenidos, pues los márgenes en aportes preferenciales desde plantas similares no son elevados, siempre que no surjan otras variables en restricción, como por ejemplo relaciones $\mathrm{H}_{2}$ /hidrocarburo que no pueden conseguirse con reciclos.

En relación al enfoque se puede decir que:

- Se han empleado técnicas bien conocidas.

- El modelo está basado en primeros principios y es fiable desde el punto de vista de las predicciones. Las hipótesis de modelado con mayor incertidumbre se refieren a los modelos de solubilidad de MP/LP, que sólo suponen en torno al $10 \%$ del total de la producción, y por tanto los resultados son válidos incluso para errores significativos.

- Se debe trabajar para mejorar el rendimiento computacional de las optimizaciones en EcosimPro ${ }^{\circledR}$, por ejemplo con entornos simultáneos de simulación-optimización como $\operatorname{CppAD}^{\circledR}$, que proporcionan derivadas exactas de primer y segundo orden y tiempos muy bajos de ejecución.

- Se puede considerar un enfoque alternativo al RTO mediante un NMPC basado en modelos de primeros principios, en particular si todas las variables de decisión pueden manipularse de forma automática.

Otros enfoques como el control self-optimizing también son interesantes, especialmente si se tiene en cuenta el hecho de que el mayor potencial de mejora corresponde a las purgas de HP a FG, que siguen un patrón lógico que podría programarse sin dificultad. 


\section{A.8.5.5- Explotación adicional del modelo y las funcionalidades de optimización}

El modelo y las funcionalidades de optimización se han explotado adicionalmente en dos aplicaciones. La primera es un estudio realizado en colaboración con el Centro de Tecnología de Repsol ${ }^{\circledR}$-CTR-, con el propósito de evaluar diferentes cambios en el diseño topológico de la red de Petronor ${ }^{\circledR}$, orientados a una minimización de la producción y reducción en el coste asociado. Estos cambios se refieren en su mayoría al aprovechamiento de corrientes ahora purgadas a FG procedentes de separadores de MP y purgas de membranas. La segunda es una simulación Qué-Pasa-Si, empleada para experimentar los efectos sobre la eficacia en el uso de $\mathrm{H}_{2}$ de diferentes estrategias operativas, complementada con un conjunto de indicadores de eficiencia (REls o KPIs) para la ayuda a la decisión en tiempo real, en el marco del proyecto europeo MORE.

\section{A.8.6- TRABAJO FUTURO}

Se detallan a continuación temas surgidos que pueden merecer un estudio posterior:

- Para aumentar la robustez frente a los errores de medida, se pueden estudiar técnicas de optimización con incertidumbre.

- Se podrían considerar los costes de compresión de gas, para evaluar si hay márgenes potenciales. En particular si se muestra que las decisiones sobre eficacia energética no pueden desacoplarse de las decisiones sobre eficacia material.

- Se podrían explorar los compromisos técnico-económicos, donde surgen comportamientos no lineales. Una operación en las plantas consumidoras a mayor $y_{\text {HPsep }}{ }^{\text {H2 }}$ conlleva beneficios adicionales por la mayor calidad y valor añadido del hidrocarburo procesado. La relación con los requerimientos de hidrógeno es no lineal, especialmente cuando se tiene en cuenta que la restricción $y_{\text {HPsep }}{ }^{\text {H2 }}$ es muy determinante para dichas necesidades de producción de hidrógeno, condicionando el potencial aprovechamiento del hidrógeno de baja pureza como aporte. La principal dificultad es cuantificar el valor añadido en el hidrocarburo

como consecuencia de la operación a mayor $y_{\text {HPsep }}{ }^{H 2}$. Estudios mediante diagramas de Pareto podrían ser útiles. 